\title{
Rate of convergence of attractors for abstract semilinear problems
}

\author{
Leonardo Pires
}



Assinatura:

\section{Leonardo Pires}

\section{Rate of convergence of attractors for abstract semilinear problems}

Doctoral dissertation submitted to the Instituto de Ciências Matemáticas e de Computação - ICMCUSP, in partial fulfillment of the requirements for the degree of the Doctorate Program in Mathematics. FINAL VERSION

Concentration Area: Mathematics

Advisor: Prof. Dr. Alexandre Nolasco de Carvalho 
Ficha catalográfica elaborada pela Biblioteca Prof. Achille Bassi e Seção Técnica de Informática, ICMC/USP, com os dados fornecidos pelo(a) autor(a)

Pires, Leonardo
Rate of convergence of attractors for abstract
semilinear problems / Leonardo Pires; orientador
Alexandre Nolasco de Carvalho. - São Carlos - SP,
2016.
135 p.
Tese (Doutorado - Programa de Pós-Graduação em
Matemática) - Instituto de Ciências Matemáticas e de
Computação, Universidade de São Paulo, 2016.
1. Attractors. 2. Rate of Convergence.
3. Parabolic Equations. 4. Nonlinear Dynamical
Systems. 5. Singular Perturbations. I. Carvalho,
Alexandre Nolasco de, orient. II. Título.




\section{Leonardo Pires}

\section{Taxa de convergência de atratores para problemas semilineares abstratos}

Tese apresentada ao Instituto de Ciências Matemáticas e de Computação - ICMC-USP, como parte dos requisitos para obtenção do título de Doutor em Ciências - Matemática. VERSÃO REVISADA

Área de Concentração: Matemática

Orientador: Prof. Dr. Alexandre Nolasco de Carvalho

USP - São Carlos

Outubro de 2016 

Para

Lorena Texluk Pires 

Os agradecimentos principais são direcionados à Alexandre Nolasco de Carvalho e Karina Schiabel Silva pela orientação acadêmica.

Agradecimentos especiais são para CAPES e CNPq pelo apoio financeiro ${ }^{1}$. 



\section{RESUMO}

PIRES,L.. Rate of convergence of attractors for abstract semilinear problems. 2016. $135 \mathrm{f}$. Doctoral dissertation (Doctorate Candidate Program in Mathematics) - Instituto de Ciências Matemáticas e de Computação (ICMC/USP), São Carlos - SP.

Neste trabalho estudamos taxa de convergência de atratores para equações parabólicas. Consideramos vários tipos de problemas onde o coeficiente de difusão apresenta perfís variados: difusão grande, difusão grande localizada e difusão grande exceto na vizinhança de um ponto onde ela torna-se pequena. Em todos os casos consideramos perturbações singulares e uma taxa de convergência para os atratores é obtida.

Palavras-chave: Atratores, Taxa de Convergência, Equações Parabólicas, Sistemas Dinâmicos não Lineares, Perturbações Singulares. 



\section{ABSTRACT}

PIRES,L.. Rate of convergence of attractors for abstract semilinear problems. 2016. $135 \mathrm{f}$. Doctoral dissertation (Doctorate Candidate Program in Mathematics) - Instituto de Ciências Matemáticas e de Computação (ICMC/USP), São Carlos - SP.

In this work we study rate of convergence of attractors for parabolic equations. We consider various types of problems where the diffusion coefficient has varied profiles: large diffusion, localized large diffusion and large diffusion except in the neighborhood of a point where it becomes small. In all cases we obtain a singular perturbation where a rate of convergence of attractors is obtained.

Key-words: Attractors, Rate of Convergence, Parabolic Equations, Nonlinear Dynamical Systems, Singular Perturbations. 

Figure 1 - Intro:Thin Domain . . . . . . . . . . . . . . . 21

Figure $2-$ Intro:Localized Diffusion . . . . . . . . . . . . . 25

Figure 3 - Phase Space ODE . . . . . . . . . . . . . . . 30

Figure 4 - Phase Space ODE $2 \ldots \ldots \ldots \ldots$

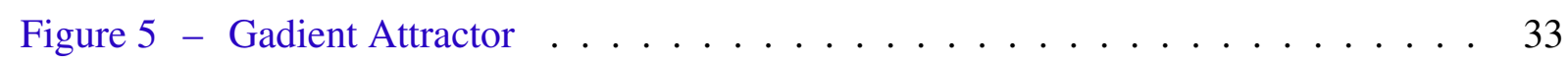

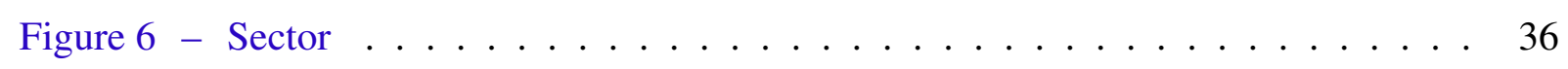

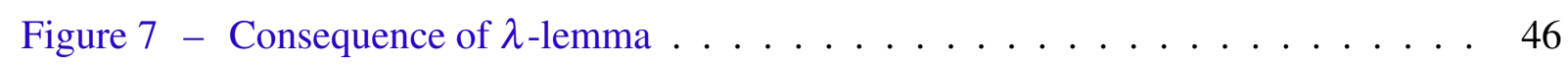

Figure 8 - Thin Domain . . . . . . . . . . . . . . . . 49

Figure $9-$ Geometry of the Phase Space . . . . . . . . . . . . . . 81

Figure 10 - Diffusion . . . . . . . . . . . . . . . . . 92

Figure 11 - Localized Diffusion . . . . . . . . . . . . . . . . 114 

$X$ - Banach space

$\mathscr{C}(X)$ - Set of all continuous transformations from $X$ into itself

$T(\cdot)$ - Semigroup

$\mathbb{R}$ - Real numbers

$\mathbb{C}$ - Complex numbers

$\operatorname{dist}_{H}(A, B)$ - Hausdorff's semidistance between $A, B \subset X$

$\mathrm{d}_{H}(A, B)$ - Hausdorff's distance between $A, B \subset X$

$\mathscr{A}$ - Global Attractor

$O D E$ - Ordinary Differential Equation

$\mathscr{E}$ - Equilibrium set

$W^{s}$ - Stable manifold

$W^{u}$ - Unstable manifold

$\mathscr{L}(X)$ - Banach space of the bounded linear operator from $X$ into itself

$\Sigma_{\varphi}-$ Sector with angle $\varphi$

$\sigma(A)-$ Spectrum set of A

$\rho(A)$ - Resolvent set of A

$T_{z} W$ - Tangent space of $W$ in $z$

LPSP — Lipschitz Shadowing Property

$\operatorname{Im}(\mu)$ - Imaginary part of $\mu$

$\operatorname{Re}(\mu)-$ Real part of $\mu$ 



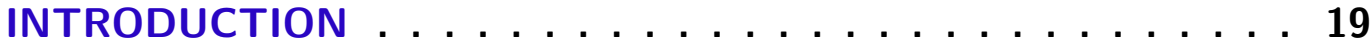

ABSTRACT THEORY . . . . . . . . . . . . . . 27

Global Attractor . . . . . . . . . . . . . . . . . . . . . 27

$2.2 \quad$ Semilinear Differential Equation . . . . . . . . . . . . 34

2.3 Rate of Convergence of Attractors . . . . . . . . . . . . . . 40

2.4 Morse-Smale Map . . . . . . . . . . . . . . . . 43

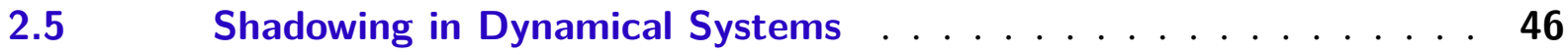

3 SEMILINEAR PROBLEMS WHOSE DYNAMICS IS DICTATED BY AN ODE ....................... 51

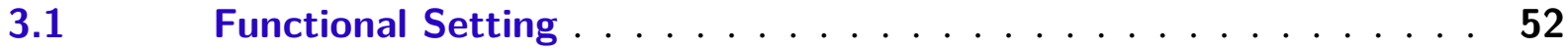

3.2 Compact Convergence . . . . . . . . . . . . . . 54

$3.3 \quad$ Invariant Manifold . . . . . . . . . . . . . . . . 61

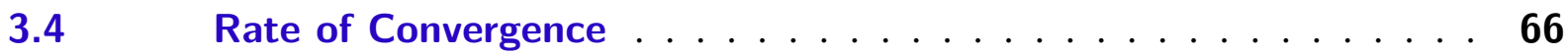

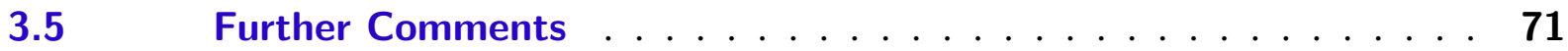

4 PARABOLIC EQUATIONS WITH LARGE DIFFUSION . . . . . . 73

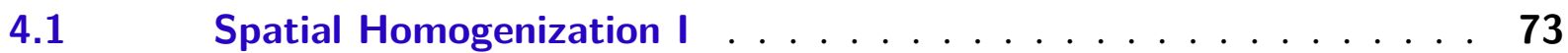

$4.2 \quad$ Spatial Homogenization II . . . . . . . . . . . . . . . . . 81

5 LARGE DIFFUSION EXCEPT IN A NEIGHBORHOOD OF A POINT 91

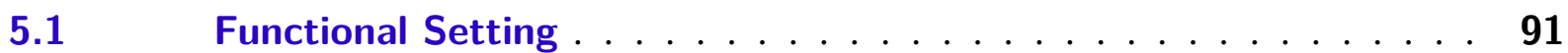

$5.2 \quad$ Elliptic Problem . . . . . . . . . . . . . . . . . . 101

$5.3 \quad$ Invariant Manifold . . . . . . . . . . . . . . 107

$5.4 \quad$ Rate of Attraction . . . . . . . . . . . . . . . . 109

6 SCALAR PARABOLIC EQUATIONS WITH LOCALIZED LARGE DIFFUSION . . . . . . . . . . . . . . . 113

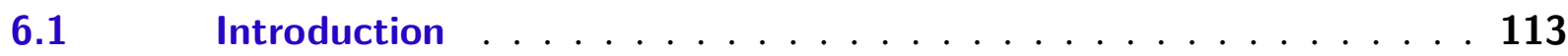

$6.2 \quad$ Elliptic Problem . . . . . . . . . . . . . . . . 117

$6.3 \quad$ Rate of Convergence of Eigenvalues and Equilibria . . . . . . . . . 121

6.4 Rate of Convergence of Invariant Manifolds . . . . . . . . . . . . 124

$6.5 \quad$ Rate of Convergence of Attractors . . . . . . . . . . . . 131 
BIBLIOGRAPHY . . . . . . . . . . . . . . . . . . . 133 
CHAPTER

1

INTRODUCTION

The study of convergence of attractors for a family of singularly perturbed parabolic equation was extensively studied in many works, see for example (HALE; RAUGEL, 1988; HALE; RAUGEL, 1989; HALE; RAUGEL, 1990; CARBONE; CARVALHO; SCHIABELSILVA, 2008; ARRIETA; CARVALHO; RODRíGUEZ-BERNAL, 2000b; BABIN; VISHIK, 1992). Since convergence of attractors provide us how the dynamics of the perturbed problem and unperturbed problem approach each other, we can consider a rate in order to estimate how fast the convergence occurs.

Inspired by the work of (BABIN; VISHIK, 1992), the authors in (ARRIETA; BEZERRA; CARVALHO, 2013) considered the semilinear parabolic problems of the form

$$
\left\{\begin{array}{l}
u_{t}^{\varepsilon}-\operatorname{div}\left(p_{\varepsilon}(x) \nabla u^{\varepsilon}\right)=f\left(u^{\varepsilon}\right), x \in \Omega, t>0, \\
u^{\varepsilon}(t, x)=0, x \in \partial \Omega, t>0 \\
u^{\varepsilon}(0, x)=u_{0}^{\varepsilon}(x),
\end{array}\right.
$$

where $\Omega \subset \mathbb{R}^{N}, N \geq 2$, is a bounded smooth domain, $\varepsilon \in[0,1]$ is a parameter, $p_{\varepsilon} \in C^{1}([0,1])$ and $f: \mathbb{R} \rightarrow \mathbb{R}$ is continuously differentiable such that

$$
\limsup _{|s| \rightarrow \infty} \frac{f(s)}{s} \leq 0 .
$$

We can write (1.1) as

$$
\left\{\begin{array}{l}
u_{t}^{\varepsilon}+A_{\varepsilon} u^{\varepsilon}=f(u), t>0 \\
u^{\varepsilon}(0)=u_{0}^{\varepsilon},
\end{array}\right.
$$

where $f$ denotes the Nemitskii functional and

$$
\begin{gathered}
A_{\varepsilon} u=-\operatorname{div}\left(p_{\varepsilon} \nabla u\right), \\
D\left(A_{\varepsilon}\right)=\left\{u \in H^{1}(\Omega):-\operatorname{div}\left(p_{\varepsilon} \nabla u\right) \in L^{2}(\Omega), u=0 \text { on } \partial \Omega\right\} .
\end{gathered}
$$


Assuming appropriated growth conditions, the problem (1.3) is well posed in the Sobolev space $H_{0}^{1}(\Omega)$, that is, the solutions generate a semigroup $T_{\mathcal{E}}(\cdot)$ with a global attractor $\mathscr{A}_{\mathcal{E}}$ for all $\varepsilon \in[0,1]$. If we assume that

$$
\left\|p_{\varepsilon}-p_{0}\right\|_{L^{\infty}} \stackrel{\varepsilon \rightarrow 0}{\longrightarrow} 0
$$

we can obtain the following estimate for the convergence of the resolvent operators

$$
\left\|A_{\varepsilon}^{-1}-A_{0}^{-1}\right\|_{\mathscr{L}\left(L^{2}, H_{0}^{1}\right)} \leq C\left\|p_{\varepsilon}-p_{0}\right\|_{L^{\infty}}
$$

where $C$ is a constant independent of $\varepsilon$.

It is also valid that for $u^{\varepsilon}, u^{0} \in H_{0}^{1}(\Omega)$,

$$
\left\|T_{\mathcal{E}}(t) u^{\varepsilon}-T_{0}(t) u^{0}\right\|_{H_{0}^{1}} \leq C e^{L t} t^{-\frac{1}{2}-\theta}\left(\left\|u^{\varepsilon}-u^{0}\right\|_{H_{0}^{1}}+\left\|p_{\varepsilon}-p_{0}\right\|_{L^{\infty}}^{2 \theta}\right), \quad 0<\theta<\frac{1}{2}, t \geq 0
$$

and

$$
\operatorname{dist}_{H}\left(T_{\varepsilon}(t) \bigcup_{\varepsilon \in\left[0, \varepsilon_{0}\right]} \mathscr{A}_{\varepsilon}, \mathscr{A}_{\varepsilon}\right) \leq C e^{-\gamma t}, \quad t \geq 0
$$

where dist $_{H}$ denotes the semidistance of Hausdorff.

Then by theory developed in (BABIN; VISHIK, 1992) we can estimate the convergence of attractor by

$$
\mathrm{d}_{H}\left(\mathscr{A}_{\mathcal{E}}, \mathscr{A}_{0}\right) \leq C\left\|p_{\mathcal{\varepsilon}}-p_{0}\right\|_{L^{\infty}}^{\frac{2 \theta \gamma}{\gamma+L}}
$$

where $C$ is a constant independent of $\varepsilon, \frac{2 \theta \gamma}{\gamma+L} \leq 1$ and $d_{H}$ denotes the distance of Hausdorff.

The estimate (1.5) does not seem optimal, in fact there is a loss when we pass the estimate (1.4) to estimate (1.5).

Thus we are faced with the question: It is possible the rate of convergence of attractors be the same rate of convergence of resolvents operators? What kind of situation can this happen?

Recently, (SANTAMARIA; ARRIETA, 2014) improve the estimate (1.5) for the situation where the limiting problem generates a Morse-Smale semigroup in the phase space and the operators are compact and have a property named gap condition. This condition ensure the existence of a finite dimension invariant manifold containing the attractor.

Consider the set

$$
Q=\left\{(x, y) \in \mathbb{R} \times \mathbb{R}^{N-1}: 0 \leq x \leq 1,|y|<1\right\}
$$

The thin domain is defined by

$$
Q_{\varepsilon}=\left\{(x, \varepsilon y) \in \mathbb{R} \times \mathbb{R}^{N-1}:(x, y) \in Q\right\}, \quad \varepsilon \in(0,1] .
$$

This domain is obtained by shrinking the set $Q$ by a factor $\varepsilon$ in the $N-1$ direction given by the variable $y$. When $\varepsilon$ goes to zero, $Q_{\varepsilon}$ tends for the one dimensional segment $(0,1)$. 


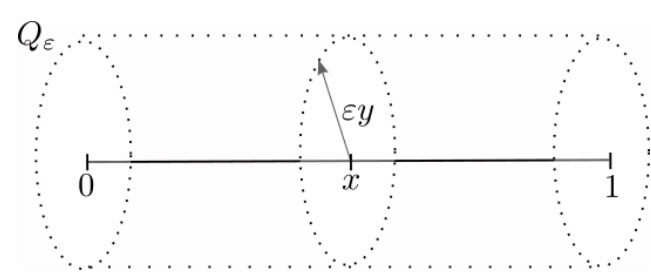

Figure 1 - Intro:Thin Domain

We consider the reaction-diffusion equation

$$
\left\{\begin{array}{l}
u_{t}-\Delta u+\alpha u=f(u), \text { in } Q_{\varepsilon} \\
\frac{\partial u}{\partial v_{\varepsilon}}=0, \text { in } \partial Q_{\varepsilon}
\end{array}\right.
$$

where $\alpha>0$ is a fixed number, $v_{\varepsilon}$ the unit outward normal to $\partial Q_{\varepsilon}$ and $f: \mathbb{R} \rightarrow \mathbb{R}$ a $C^{2}$-function satisfying

(i) $\left|f^{\prime}(s)\right| \leq C\left(1+|s|^{\rho-1}\right), s \in \mathbb{R}$, for some $\rho>1$,

(ii) $f(s) s \leq 0,|s| \geq M$, for some $M>0$.

The limiting problem of (1.6) as $\varepsilon \rightarrow 0$ is given by

$$
\left\{\begin{array}{l}
u_{t}-u_{x x}+\alpha u=f(u), x \in(0,1), \\
u_{x}(0)=u_{x}(1)=0
\end{array}\right.
$$

The equation (1.6) is well posed in $H^{1}\left(Q_{\varepsilon}\right)$ and the equation (1.7) is well posed in the space $H^{1}(0,1)$, that is, if we define the operators $A_{\varepsilon}=-\Delta u+\alpha u$ and $A_{0} u=-u_{x x}+\alpha u$, the solutions of $u_{t}+A_{\varepsilon} u^{\varepsilon}=f\left(u^{\varepsilon}\right)$ generate a semigroup $T_{\varepsilon}(\cdot)$, for all $\varepsilon \in[0,1]$, and this semigroups have a global attractor $\mathscr{A}_{\varepsilon}$, for all $\varepsilon \in[0,1]$. Moreover $T_{0}(\cdot)$ is a Morse-Smale semigroup.

In order to understand the attractor $\mathscr{A}_{0}$ in $H^{1}\left(Q_{\varepsilon}\right)$, we define $E_{\varepsilon}: H^{1}(0,1) \rightarrow H^{1}\left(Q_{\varepsilon}\right)$ by $\left(E_{\varepsilon} u\right)(x, y)=u(x)$, for all $u \in H^{1}(0,1)$.

Assuming that the equilibrium points of (1.7) are hyperbolic, we have the following results see (SANTAMARIA; ARRIETA, 2014),

(i) $\left\|A_{\varepsilon}^{-1}-E_{\varepsilon} A_{0}^{-1}\right\|_{\mathscr{L}\left(L^{2}, H^{1}\right)} \leq C \varepsilon$;

(ii) $\left\|T_{\varepsilon}(1)-E_{\varepsilon} T_{0}(1)\right\|_{\mathscr{L}\left(H^{1}\right)} \leq C \varepsilon|\log (\varepsilon)|$;

(iii) There is a finite dimensional invariant manifold $\mathscr{M}_{\varepsilon}$ given by graph of Lipschitz functions $s_{\varepsilon}$ such that $\mathscr{A}_{\varepsilon} \subset \mathscr{M}_{\varepsilon}$ and the flow can be reduced to finite dimension, that is, we can consider an ODE generating a semigroup $\bar{T}_{\mathcal{E}}(\cdot)$ in $\mathbb{R}^{m}$ with an attractor $\overline{\mathscr{A}}_{\mathcal{\varepsilon}}$, where $m=\operatorname{dim}\left(\mathscr{M}_{\varepsilon}\right)$. Moreover the following estimates hold 
(a) $\left\|\bar{T}_{\mathcal{E}}(1)-\bar{T}_{0}(1)\right\|_{\mathscr{L}\left(\mathbb{R}^{m}\right)} \leq C \varepsilon|\log (\varepsilon)|$;

(b) $\sup _{v \in \mathbb{R}^{m}}\left\|s_{\mathcal{\varepsilon}}(v)-s_{0}(v)\right\|_{H^{1}} \leq C \varepsilon|\log (\varepsilon)|$.

Therefore the convergence of attractors of (1.6) and (1.7) can be estimated by

$$
\mathrm{d}_{H}\left(\mathscr{A}_{\varepsilon}, E_{\varepsilon} \mathscr{A}_{0}\right) \leq C \varepsilon^{\frac{N+1}{2}}|\log (\varepsilon)|
$$

where $C$ is a constant independent of $\varepsilon$.

Note that the estimate (1.8) improves the estimate (1.5) but still the rate does not seem optimal. There is the factor log that represent a loss in the rate of convergence of attractors.

The aim of this work is to find situations where we can get an optimal rate for the convergence of attractors. In this direction, we consider, see the Chapter 4, the following equation

$$
\left\{\begin{array}{l}
u_{t}^{\varepsilon}-\frac{1}{\varepsilon} u_{x x}^{\varepsilon}+u^{\varepsilon}=f\left(u^{\varepsilon}\right), \quad x \in(0,1), t>0 \\
u_{x}^{\varepsilon}=0, \quad x \in\{0,1\} \\
u^{\varepsilon}(0)=u_{0}^{\varepsilon}
\end{array}\right.
$$

where $0<\varepsilon \leq \varepsilon_{0}$ and $f: \mathbb{R} \rightarrow \mathbb{R}$ is continuously differentiable satisfying (1.2). Taking the average on $(0,1)$, the limiting problem as $\varepsilon$ goes to zero is given by ordinary differential equation

$$
\left\{\begin{array}{l}
\dot{u}^{0}+u^{0}=f\left(u^{0}\right), \quad t>0, \\
u^{0}(0)=u_{0}^{0}
\end{array}\right.
$$

We define the operator $A_{\varepsilon}: D\left(A_{\varepsilon}\right) \subset L^{2}(0,1) \rightarrow L^{2}(0,1)$ by

$$
D\left(A_{\varepsilon}\right)=\left\{u \in H^{2}(\Omega): u_{x}^{\varepsilon}(0)=0=u_{x}^{\varepsilon}(1)\right\}, \quad A_{\varepsilon} u=-\frac{1}{\varepsilon} u_{x x}+u .
$$

We denote $L_{(0,1)}^{2}=\left\{u \in H^{1}(\Omega): u_{x}=0\right.$ in $\left.(0,1)\right\}$ and we define the operator $A_{0}: L_{(0,1)}^{2} \rightarrow$ $L^{2}(0,1)$ by $A_{0} u=u$. Therefore we can formulate the well posedness of the problems (1.9) and (1.10) in the abstract fractional power space $X_{\varepsilon}^{\frac{1}{2}}=D\left(A_{\varepsilon}^{\frac{1}{2}}\right)$ (see (HENRY, 1980)). If we consider an appropriated projection $P$ from $X_{\mathcal{\varepsilon}}^{\frac{1}{2}}$ onto $L_{(0,1)}^{2}$, it is valid the following estimate

$$
\left\|A_{\varepsilon}^{-1}-A_{0}^{-1} P\right\|_{\mathscr{L}\left(L^{2}, X_{\varepsilon}^{\frac{1}{2}}\right)} \leq C \varepsilon^{\frac{1}{2}}
$$

where $C$ is a constant independent of $\varepsilon$. Moreover there is a finite dimensional invariant manifold $\mathscr{M}_{\varepsilon}$ given by graph of Lipschitz functions such that $\mathscr{A}_{\varepsilon} \subset \mathscr{M}_{\varepsilon}$ and the flow can be reduced to finite dimension. The convergence of attractors can be estimated by

$$
\mathrm{d}_{H}\left(\mathscr{A}_{\varepsilon}, \mathscr{A}_{0}\right) \leq C \varepsilon^{\frac{1}{2}}
$$

where $C$ is a constant independent of $\varepsilon$. 
Note that in this case the rate of attractors (1.12) is the same rate of the resolvents operators (1.11). This result improve the results obtanid previously. Therefore we investigate a class of parabolic problems whose asymptotic behavior is described by a system of ordinary differential equation. We prove that in this situation we have the optimal rate for the convergence of attractors, in the sense that, the rate of convergence attractors is the same rate of convergence of resolvents operators.

Let $X_{0}$ be a finite dimensional Hilbert space with $\operatorname{dim}\left(X_{0}\right)=n$, for some positive integer $n$, and let $A_{0}: X_{0} \rightarrow X_{0}$ be an invertible bounded linear operator. Consider the following system of ordinary differential equation

$$
\left\{\begin{array}{l}
\dot{u}^{0}+A_{0} u^{0}=f_{0}\left(u^{0}\right) \\
u^{0}(0)=u_{0}^{0} \in X_{0}
\end{array}\right.
$$

where $f_{0}: X_{0} \rightarrow X_{0}$ is continuously differentiable.

Let $\left\{X_{\varepsilon}\right\}_{\varepsilon \in\left(0, \varepsilon_{0}\right]}, \varepsilon_{0}>0$, be a family of separable Hilbert spaces and let $\left\{A_{\varepsilon}\right\}_{\varepsilon \in\left(0, \varepsilon_{0}\right]}$ be a family of invertible linear operators such that, for each $\varepsilon \in\left(0, \varepsilon_{0}\right], A_{\varepsilon}: D\left(A_{\varepsilon}\right) \subset X_{\varepsilon} \rightarrow X_{\varepsilon}$ is self-adjoint, positive and has compact resolvent. Consider the following abstract parabolic problem,

$$
\left\{\begin{array}{l}
u_{t}^{\varepsilon}+A_{\varepsilon} u^{\varepsilon}=f_{\varepsilon}\left(u^{\varepsilon}\right) \\
u^{\varepsilon}(0)=u_{0}^{\varepsilon} \in X_{\varepsilon}^{\alpha},
\end{array}\right.
$$

where $f_{\varepsilon}: X_{\mathcal{\varepsilon}}^{\alpha} \rightarrow X_{\mathcal{\varepsilon}}$ is continuously differentiable and $X_{\mathcal{\varepsilon}}^{\alpha}$ is the fractional power space associated with $A_{\varepsilon}$.

We assume that there are two positive increasing functions $\tau, \rho:\left[0, \varepsilon_{0}\right] \rightarrow[0, \infty)$ such that $\tau(0)=0=\rho(0)$,

$$
\left\|A_{\varepsilon}^{-1}-E_{\varepsilon} A_{0}^{-1} M_{\varepsilon}\right\|_{\mathscr{L}\left(X_{\varepsilon}, X_{\varepsilon}^{\alpha}\right)} \leq \tau(\varepsilon)
$$

and

$$
\left\|f_{\varepsilon}\left(u^{\varepsilon}\right)-E_{\varepsilon} f_{0}\left(u^{0}\right)\right\|_{X_{\varepsilon}} \leq C\left\|u^{\varepsilon}-E_{\varepsilon} u^{0}\right\|_{X_{\varepsilon}^{\alpha}}+\rho(\varepsilon), u^{\varepsilon} \in X_{\varepsilon}^{\alpha}, u^{0} \in X_{0},
$$

where $C$ is a constant independent of $\varepsilon$ and $E_{\varepsilon}, M_{\varepsilon}$ are mapping that enable us consider the problems in the same space.

Now we can state our main result in this work: the problems (1.13) and (1.14) are well posed in $X_{0}$ and $X_{\varepsilon}^{\alpha}$ respectively, that is, there are attractors $\mathscr{A}_{0} \subset X_{0}$ and $\mathscr{A}_{\varepsilon} \subset X_{\varepsilon}^{\alpha}$ for (1.13) and (1.14). There also is a finite dimensional invariant manifold $\mathscr{M}_{\varepsilon}$ given by graph of Lipschitz functions $s_{\varepsilon}$ such that $\mathscr{A}_{\varepsilon} \subset \mathscr{M}_{\varepsilon}$ and the flow can be reduced to finite dimension. Moreover the convergence of attractors can be estimated by

$$
\mathrm{d}_{\varepsilon}\left(\mathscr{A}_{\varepsilon}, E_{\varepsilon} \mathscr{A}_{0}\right) \leq C(\tau(\varepsilon)+\rho(\varepsilon)),
$$

where $d_{\varepsilon}$ represents the Hausdorff distance in $X_{\varepsilon}^{\alpha}$. 
Note that we do not have loss when we pass the convergence of resolvents operators (1.15) and nonlinearities convergence (1.16) to convergence of attractors (1.17). Therefore for the class where the dynamics can be described by a system of ODE we obtain the optimal rate. The main idea for the proof is that the asymptotic behavior is dictated by an ODE, that is, the phase space of the limiting problem is finite dimensional according to (1.10).

When the limiting phase space is infinite dimensional we can not obtain the optimal rate as above, but the theory developed in (SANTAMARIA; ARRIETA, 2014) enable us treat more general problems. We consider parabolic equations with localized large diffusion.

Consider the scalar parabolic problem

$$
\left\{\begin{array}{l}
u_{t}^{\varepsilon}-\left(p_{\varepsilon}(x) u_{x}^{\varepsilon}\right)_{x}+(\lambda+c(x)) u^{\varepsilon}=f\left(u^{\varepsilon}\right), \quad 0<x<1, t>0 \\
u_{x}^{\varepsilon}(0)=u_{x}^{\varepsilon}(1)=0, \quad t>0 \\
u^{\varepsilon}(0)=u_{0}^{\varepsilon}
\end{array}\right.
$$

where $\varepsilon \in\left(0, \varepsilon_{0}\right]$ is a parameter $\left(0<\varepsilon_{0}<1\right), c \in C^{1}([0,1])$ is such that $c(x)=c_{\Omega_{0}}$ for all $x \in\left(x_{1}, x_{2}\right)$ where $c_{\Omega_{0}}$ is a constant, $f \in C^{2}(\mathbb{R})$ and $\lambda \in \mathbb{R}$ is such that

$$
0<m_{0} \leq \min _{x \in[0,1]} c(x)+\lambda
$$

for some positive constant $m_{0}$.

To describe the coefficients $p_{\varepsilon}$, let $0=x_{0}<x_{1}<x_{2}<x_{3}=1$ be a partition of the interval $\Omega=(0,1)$. We assume the diffusion is very large in the open interval $\Omega_{0}=\left(x_{1}, x_{2}\right)$ and converges uniformly to $p_{0} \in C^{2}\left(\Omega_{1}\right)$ in the $\Omega_{1}=\left[0, x_{1}\right] \cup\left[x_{2}, 1\right]$ as $\varepsilon$ approaches to zero. More precisely, for $\varepsilon \in\left(0, \varepsilon_{0}\right], p_{\varepsilon} \in C^{2}([0,1])$ satisfies the following conditions (see Fig 2).

$$
\left\{\begin{array}{l}
p_{\varepsilon} \stackrel{\varepsilon \rightarrow 0}{\longrightarrow} p_{0}, \quad \text { uniformly in } \Omega_{1}, \\
p_{\varepsilon}(x) \geq \frac{1}{\varepsilon} \quad \text { in }\left[x_{1}+\varepsilon, x_{2}-\varepsilon\right] \\
m_{0} \leq p_{\varepsilon} \quad \text { in } \Omega \quad \text { and } \quad m_{0} \leq p_{0} \quad \text { in } \Omega_{1}
\end{array}\right.
$$

The limiting problem of (1.18) as $\varepsilon \rightarrow 0$ is given by

$$
\left\{\begin{array}{l}
u_{t}^{0}-\left(p_{0}(x) u_{x}^{0}\right)_{x}+(\lambda+c(x)) u^{0}=f\left(u^{0}\right), \quad x \in \Omega_{1}, t>0 \\
u_{x}^{0}(0)=u_{x}^{0}(1)=0, \quad t>0 \\
\left.u^{0}\right|_{\Omega_{0}}=u_{\Omega_{0}}^{0} \\
\dot{u}_{\Omega_{0}}^{0}+\left(\lambda+c_{\Omega_{0}}\right) u_{\Omega_{0}}^{0}=f\left(u_{\Omega_{0}}^{0}\right), x \in \Omega_{0}, \\
u^{0}(0)=u_{0}^{0}
\end{array}\right.
$$

where $u_{0}^{0}=\lim _{\varepsilon \rightarrow 0} u_{0}^{\varepsilon}$ is constant on $\Omega_{0}$. 


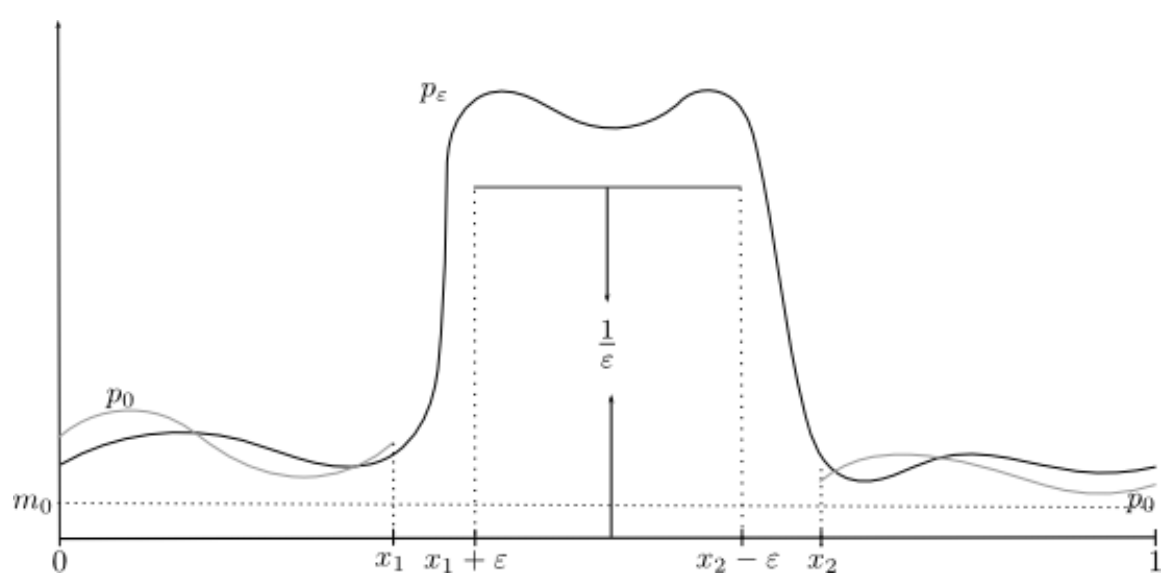

Figure 2 - Intro:Localized Diffusion

In order to write (1.18) and (1.19) abstractly in a suitable natural energy space we introduce some more terminology. We define the operator $A_{\varepsilon}: \mathscr{D}\left(A_{\varepsilon}\right) \subset L^{2}(0,1) \rightarrow L^{2}(0,1)$ by

$$
\mathscr{D}\left(A_{\varepsilon}\right)=\left\{u \in H^{2}(0,1) ; u_{x}(0)=u_{x}(1)=0\right\} \quad \text { and } \quad A_{\varepsilon} u=-\left(p_{\varepsilon} u_{x}\right)_{x}+(\lambda+c) u \text {. }
$$

We denote

$$
\begin{gathered}
L_{\Omega_{0}}^{2}(0,1)=\left\{u \in L^{2}(0,1) ; u \text { is constant a.e. in } \Omega_{0}\right\} \\
H_{\Omega_{0}}^{1}(0,1)=\left\{u \in H^{1}(0,1) ; u_{x}=0 \text { in } \Omega_{0}\right\}
\end{gathered}
$$

and we define the operator $A_{0}: \mathscr{D}\left(A_{0}\right) \subset L_{\Omega_{0}}^{2}(0,1) \rightarrow L_{\Omega_{0}}^{2}(0,1)$ by

$$
\begin{gathered}
\mathscr{D}\left(A_{0}\right)=\left\{u \in H_{\Omega_{0}}^{1}(0,1) ;-\left(p_{0} u_{x}\right)_{x} \in L^{2}\left(\Omega_{1}\right), u_{x}(0)=u_{x}(1)=0\right\} ; \\
A_{0} u=\left[-\left(p_{0} u_{x}\right)_{x}+(\lambda+c) u\right] \chi_{\Omega_{1}}+\left[\left(\lambda+c_{\Omega_{0}}\right) u_{\Omega_{0}}\right] \chi_{\Omega_{0}} .
\end{gathered}
$$

If we denote the Nemitskii functional of $f$ by the same notation $f$, then (1.18) and (1.19) can be written as

$$
\left\{\begin{array}{l}
u_{t}^{\varepsilon}+A_{\varepsilon} u^{\varepsilon}=f\left(u^{\varepsilon}\right), \\
u^{\varepsilon}(0)=u_{0}^{\varepsilon} \in X_{\mathcal{\varepsilon}}^{\frac{1}{2}}, \quad \varepsilon \in\left[0, \varepsilon_{0}\right]
\end{array}\right.
$$

where $X_{\mathcal{\varepsilon}}^{\frac{1}{2}}$ is the fractional power space associated with $A_{\mathcal{\varepsilon}}$.

We prove in the Chapter 6 that the rate of convergence of resolvent operator is given by

$$
\left\|A_{\varepsilon}^{-1}-A_{0}^{-1}\right\|_{\mathscr{L}\left(L_{\Omega_{0}}^{2}, X_{\varepsilon}^{\frac{1}{2}}\right)} \leq C\left(\left\|p_{\varepsilon}-p_{0}\right\|_{L^{\infty}\left(\Omega_{1}\right)}+\varepsilon\right)^{\frac{1}{2}},
$$

where $C$ is a positive constant independent of $\varepsilon$. Moreover, the problems (1.18) and (1.19) are well posed in $X_{0}^{\frac{1}{2}}$ and $X_{\varepsilon}^{\frac{1}{2}}$ respectively, that is, there are attractors $\mathscr{A}_{0} \subset X_{0}^{\frac{1}{2}}$ and $\mathscr{A}_{\varepsilon} \subset X_{\varepsilon}^{\frac{1}{2}}$ for (1.18) and (1.19). There also is a finite dimensional invariant manifold $\mathscr{M}_{\varepsilon}$ such that $\mathscr{A}_{\varepsilon} \subset \mathscr{M}_{\varepsilon}$ 
and the flow can be reduced to finite dimension. Moreover the convergence of attractors can be estimated by

$$
\mathrm{d}_{H}\left(\mathscr{A}_{\varepsilon}, \mathscr{A}_{0}\right) \leq C\left(\left\|p_{\varepsilon}-p_{0}\right\|_{L^{\infty}\left(\Omega_{1}\right)}+\varepsilon\right)^{\frac{1}{2}}\left|\log \left(\left\|p_{\varepsilon}-p_{0}\right\|_{L^{\infty}\left(\Omega_{1}\right)}+\varepsilon\right)\right|
$$

To make a better presentation we divide this work in the following way: in the Chapter 2 we present all preliminary abstract theory necessary. The Chapter 3 develops the theory of optimal rate of convergence for problems whose limiting problems is an ordinary differential equation. We reserve the Chapters 4 and 5 for applications of the results in the Chapter 3. Finally in the Chapter 6 we consider a more general scalar parabolic equations with localized large diffusion.

PAPERS FROM PHD THESIS

- CARVALHO,A.N.;SCHIABEL-SILVA,K.;PIRES,L. Rate of convergence of attractors for semilinear singularly perturbed problems: scalar parabolic equations with localized large diffusion - arXiv:1606.03771

- CARVALHO,A.N.;PIRES,L. Rate of convergence of attractors for semilinear singularly perturbed problems: parabolic equations with large diffusion - arXiv:1607.01349

- CARVALHO,A.N.;PIRES,L. Rate of Convergence of Attractors for Singularly Perturbed Semilinear Problems - arXiv:1606.03772

Thesis prepared following format and structure defined by publication "Diretrizes para apresentação de dissertações e teses da USP: documento eletrônico e impresso. Parte I (ABNT)" published by the Integrated Library System (SIBi) USP.

http://www.icmc.usp.br/Portal/conteudo/237/13/matematica 
In this chapter we treat some of the abstract results that we will use in the next chapters. In the first sections we will recall the global attractor notion for a semigroup, rate of convergence for the continuity of attractors and some aspects of semilinear parabolic equations. In the final sections we present the Morse-Smale semigroups and how the property of Lipschitz Shadowing can be used to improve the rate of convergence of attractors. All results presented here can be found in the references (BABIN; VISHIK, 1992; CARVALHO; LANGA; ROBINSON, 2010; HALE, 1988; HENRY, 1980) and (ROBINSON, 2001).

\subsection{Global Attractor}

We begin with precise definition of the notion of semigroup. The dynamics occurs in a phase space $X$, which represents all possible states of the system. We will take the phase space to be a Banach space $\left(X,\|\cdot\|_{X}\right)$ and we will denote by $\mathscr{C}(X)$ the set of all continuous transformations from $X$ into itself.

Definition 2.1.1. A continuous semigroup is a one parameter family $\{T(t): t \geq 0\}$ in $\mathscr{C}(X)$ such that

(i) $T(0)=I$,

(ii) $T(t+s)=T(t) T(s)$ for all $t, s \geq 0$,

(iii) $[0, \infty) \times X \ni(t, u) \rightarrow T(t) u \in X$ is continuous.

For simplicity we will refer to the semigroup $T(\cdot)$ rather than the continuous semigroup $\{T(t): t \geq 0\}$.

A solution of $T(\cdot)$ corresponding to the initial condition $u_{0} \in X$ is the mapping $t \rightarrow T(t) u_{0}$ from $[0, \infty)$ into $X$. We also consider solutions defined for all time. 
Definition 2.1.2. A continuous function $\xi(\cdot): \mathbb{R} \rightarrow X$ is a global solution for $T(\cdot)$ if it satisfies $T(t) \xi(s)=\xi(t+s)$ for all $s \in \mathbb{R}$ and $t \geq 0$. The orbit of a global solution is

$$
\gamma(\xi(\cdot))=\bigcup_{t \in \mathbb{R}} \xi(t)
$$

The natural example of semigroup is given by a initial value problem.

Example 2.1.3. Consider the initial value problem

$$
\left\{\begin{array}{l}
\dot{x}=f(x) \\
x(0)=x_{0} \in \mathbb{R}^{n}
\end{array}\right.
$$

where $f: \mathbb{R}^{n} \rightarrow \mathbb{R}^{n}$ is a continuously differentiable function. In this situation, for each $x_{0} \in \mathbb{R}^{n}$, there are $\tau>0$ and a continuously differentiable function $[0, \tau) \ni t \rightarrow \xi(t) \in \mathbb{R}^{n}$ such that $\xi(0)=x_{0}$ and $\dot{\xi}(t)=f(\xi(t))$ for all $t \in[0, \tau)$. The function $\xi$ is called solution of the initial value problem. With some standard condition (see (HALE, 2009)) we can assume that the solution $\xi$ is uniquely defined for all $t>0$ and then we denote it by $x\left(t, 0, x_{0}\right)$. Now define

$$
\left\{\begin{array}{l}
T(0)=I \\
T(t) y=x(t, 0, y), \quad t>0, y \in \mathbb{R}^{n}
\end{array}\right.
$$

It follows that $T(\cdot)$ is a continuous semigroup in $\mathbb{R}^{n}$.

The next example can be found in (HENRY, 1994b).

Example 2.1.4. Let $u_{n}(x)=(2 / \pi)^{\frac{1}{2}} \sin (n x), x \in[0, \pi], n \in \mathbb{N}$. We have

$$
\int_{0}^{\pi} u_{n} u_{m}=\delta_{n m}=\left\{\begin{array}{lc}
0, & n \neq m \\
1 & n=m
\end{array}\right.
$$

Define the projection $E_{n} \in \mathscr{L}\left(L^{2}(0, \pi)\right)$ by

$$
\left(E_{n} \psi\right)(x)=u_{n}(x) \int_{0}^{\pi} \psi(y) u_{n}(y) d y .
$$

We have

$$
\left\{\begin{array}{l}
\left\|E_{n}\right\| \mathscr{L}=1 \\
E_{n}^{2}=E_{n} \\
E_{n} E_{m}=E_{m} E_{n}=0, \quad n \neq m .
\end{array}\right.
$$

Finally, we define

$$
T(t) \psi=\sum_{n=1}^{\infty} e^{-n^{2} t} E_{n} \psi, \quad t \geq 0, \quad \psi \in L^{2}(0, \pi) .
$$

If $t>0$ it is clear that $T(t) \in \mathscr{L}\left(L^{2}(0, \pi)\right)$, since the series converge in the norm topology of $\mathscr{L}\left(L^{2}(0, \pi)\right)$, and in fact $t \rightarrow T(t)$ is easily seen to be continuous in the uniform topology 
when $t>0$, since the series converges uniformly for $t \geq \delta$, for any $\delta$. The orthogonality of the projections show, when the series are multiplied, that $T(t+s)=T(t) T(s)$ for all $t, s>0$. For $\psi \in L^{2}(0, \pi), \sum_{n=1}^{N} E_{n} \psi$ is the $N$ partial sum of the Fourier sine series for $\psi$, which converges to $\psi$ in $L^{2}(0, \pi)$ when $N \rightarrow \infty$, so $T(0)=I$. Thus $T(\cdot)$ is a semigroup in $L^{2}(0, \pi)$. Note that $T(t)$ is linear for each $t \geq 0$. The linear semigroup will be considered in the Section 2.2.

Now we recall the notion of global attractor for continuous semigroups. Ideally, the attractor of a given semigroup should contain all asymptotic dynamics, therefore lead a reasonable understanding of the asymptotic behavior of the dynamical system.

We denote by $\operatorname{dist}_{H}(A, B)$ the Hausdorff semidistance between $A, B \subset X$, defined as

$$
\operatorname{dist}_{H}(A, B)=\sup _{a \in A} \inf _{b \in B}\|a-b\|_{X},
$$

and we define the symmetric Hausdorff metric by

$$
\mathrm{d}_{H}(A, B)=\max \left\{\operatorname{dist}_{H}(A, B), \operatorname{dist}_{H}(B, A)\right\} .
$$

Note that $\operatorname{dist}_{H}(A, B)=0$ implies only $\bar{A} \subset \bar{B}$, where $\bar{M}$ denotes the closure of $M$ in $X$. But if $B$ is closed then $\operatorname{dist}_{H}(A, B)=0$ implies $A \subset B$.

In the Chapters 3, 4 and 5 we consider family of Banch spaces $X_{\varepsilon}$ indexed with a parameter $\varepsilon$, in this situation we denote the Hausdorff semidistance and the Hausdorff distance respectively by $\operatorname{dist}_{\varepsilon}$ and $\mathrm{d}_{\varepsilon}$.

Definition 2.1.5. A set $\mathscr{A} \subset X$ is said to be a global attractor for a semigroup $T(\cdot)$ if

(i) $\mathscr{A}$ is compact,

(ii) $\mathscr{A}$ is invariant, that is, $T(t) \mathscr{A}=\mathscr{A}$, for all $t \geq 0$,

(iii) $\mathscr{A}$ attracts bounded subsets of $X$, that is, $\operatorname{dist}_{H}(T(t) B, \mathscr{A}) \rightarrow 0$ as $t \rightarrow \infty$, for each bounded subset $B \subset X$.

This definition in fact yields the minimal compact set that attracts each bounded subset of $X$ and the maximal closed and bounded invariant set. In additional, the global attractor can be characterised as the collection of all globally defined bounded solution.

Theorem 2.1.6. If a semigroup $T(\cdot)$ has a global attractor $\mathscr{A}$, then

$$
\mathscr{A}=\{v \in X: \text { there is a bounded global solution } \xi: \mathbb{R} \rightarrow X \text { with } \xi(0)=v\} .
$$

Proof. That any $u_{0} \in \mathscr{A}$ lies on a bounded global solution is as consequence of the invariance. In fact, for $t \geq 0$ define $\xi(t)=T(t) u_{0}$ and for $t<0$ define $\xi(t)=T(t+n) u_{-n}$ for $-n \leq t \leq-(n-1)$. Conversely, if $\xi(\cdot)$ is a bounded solution then $\mathscr{A}$ attracts $\gamma(\xi(\cdot))$. Since $\xi(0)=T(t) \xi(-t)$, it follows that $\operatorname{dist}_{H}(\xi(0), \mathscr{A}) \leq \operatorname{dist}_{H}(T(t) \gamma(\xi(\cdot)), \mathscr{A})$ for any $t \geq 0$, and $\operatorname{so~dist~}_{H}(\xi(0), \mathscr{A})=0$. Since $\mathscr{A}$ is closed we have $\xi(0) \in \mathscr{A}$. 
If the semigroup arises from a differential equation or a partial differential equation the Theorem 2.1.6 gives an analytic, rather than dynamical, characterisation of the global attractor.

An attractor can be a point, a finite set of points, a curve, a manifold, or even a complicated set. Other types of attractors are considered in the literature, we can find the notions of point attractor, random attractor and pullback attractor. They all have the same feature, to dictate the dynamics of the system. We consider only global attractor.

We give two example of attractor for ordinary differential equation in $\mathbb{R}^{2}$ where the phase space is geometrically represented in the Figures 3 and 4.

Example 2.1.7. Consider the scalar ODE

$$
\dot{u}=u\left(1-u^{2}\right) .
$$

The global attractor is $[-1,1]$ since the global attractor must attract the interval $(-\varepsilon, \varepsilon)$ for any $\varepsilon>0$, see Figure 3 .

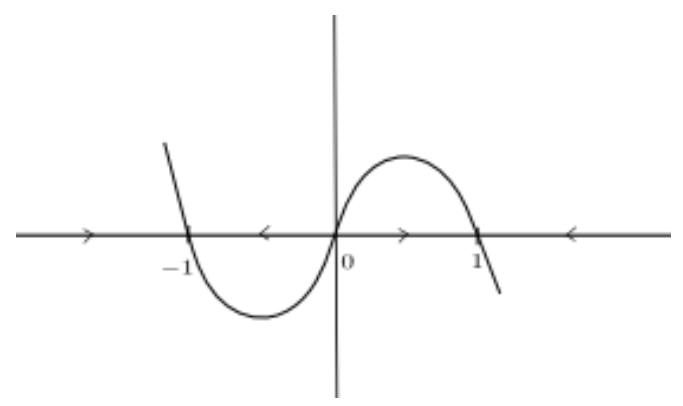

Figure 3 - Phase Space ODE

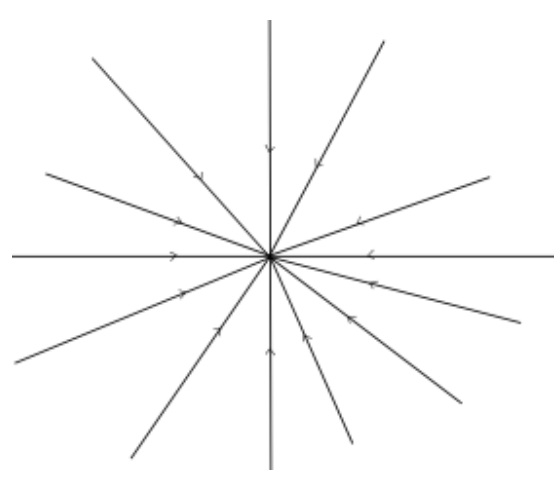

Figure 4 - Phase Space ODE 2

Example 2.1.8. Consider the two dimensional ODE

$$
\dot{u}=\left(\begin{array}{cc}
-1 & 0 \\
0 & -1
\end{array}\right) u .
$$


For each $\left(u_{1}, u_{2}\right) \in \mathbb{R}^{2}$ the solution is given by $\xi(t)=\left(u_{1} e^{-t}, u_{1} e^{-t}\right)$, for $t \in \mathbb{R}$. The global attractor is the origin $(0,0)$ since the global attractor must attract each point $\left(u_{1}, u_{2}\right) \in \mathbb{R}^{2}$, see Figure 4.

We want to give some existence results for attractor, they all require some boundedness and compactness properties of the semigroup. We start with some definitions.

Definition 2.1.9. A semigroup $T(\cdot)$ is dissipative if it possesses a compact absorbing set $A$; that is, for any bounded set $B \subset X$ there is a $t_{B}>0$ such that

$$
T(t) B \subset A, \text { for all } t \geq t_{B}
$$

Definition 2.1.10. A semigroup $T(\cdot)$ is said to be asymptotically compact if for each bounded sequence $\left\{x_{k}\right\} \in X$ and $\left\{t_{k}\right\} \geq 0$ with $t_{k} \rightarrow \infty$ as $k \rightarrow \infty$, the sequence $\left\{T\left(t_{k}\right) x_{k}\right\}$ has a convergence subsequence.

Definition 2.1.11. A semigroup $T(\cdot)$ is said to be bounded if $\cup_{t \geq 0} T(t) B$ is bounded for every bounded subset $B$ in $X$.

Theorem 2.1.12. Let $T(\cdot)$ be a bounded semigroup. The following statements are equivalent:

(i) $T(\cdot)$ has a global attractor $\mathscr{A}$.

(ii) $T(\cdot)$ has a compact attracting set $K$.

(iii) $T(\cdot)$ has a bounded attracting set and is asymptotically compact.

(iv) $T(\cdot)$ is asymptotically compact and there is a bounded set that attracts points.

Proof. All proof details can be found in (CARVALHO; LANGA; ROBINSON, 2010). We just state here that if $T(\cdot)$ has a global attractor, then the closure of a neighborhood of its attractor is a compact attracting set. Conversely if $T(\cdot)$ has a compact attracting set then we define

$$
\mathscr{A}=\overline{\cup\{\omega(B): B \subset X, B \text { bounded }\}},
$$

where

$$
\omega(B)=\bigcap_{t \geq 0} \overline{\bigcup_{s \geq t} T(s) B}
$$

is the $\omega$-limit of $B$. If $T(\cdot)$ is asymptotically compact then $\omega(B)$ is nonempty, compact, invariant and attracts $B$.

Example 2.1.13. The Lorenz equation is given by

$$
\left\{\begin{array}{l}
\dot{x}=-\sigma x+\sigma y \\
\dot{y}=-r x-y-x z \\
\dot{z}=-x y-b z
\end{array}\right.
$$


with $\sigma, r$ and $b$ all positive. Since bounded sets are compact in $\mathbb{R}^{3}$, all we need to show is the existence of a bounded absorbing set. In fact we need to show an uniform bound on each individual solution. We will see that a large enough sphere centred on $(0,0, r+\sigma)$ is absorbing. In fact, consider

$$
V(x, y, z)=x^{2}+y^{2}+(z-r-\sigma)^{2}
$$

which satisfies

$$
\dot{V} \leq \alpha V+b(r+\sigma)^{2}
$$

where $\alpha=\min (2 \sigma, 2, b)$. We can prove that

$$
V(t) \leq \frac{2 b(r+\sigma)^{2}}{\alpha}
$$

when $t$ is large enough, see (ROBINSON, 2001).

We now want to examine the attractor itself in more detail, investigating its structure. For gradient system we will see that the attractor consists of all complete bounded orbits and contains the unstable manifolds of all fixed points. This give us a better idea of the kind of dynamics we can expect to understand if we restrict our attention to the attractor.

Definition 2.1.14. We say that $x^{*} \in X$ is an equilibrium point for the semigroup $T(\cdot)$ if it is a fixed point for the map $T(t)$ for each $t \geq 0$, that is, $T(t) x^{*}=x^{*}$ for each $t \geq 0$. We denote by $\mathscr{E}$ the set of all equilibrium points for $T(\cdot)$.

Definition 2.1.15. A semigroup $T(\cdot)$ is said to be gradient if there is a continuous function $V: X \rightarrow \mathbb{R}$, a Lyapunov function, with the following properties:

(i) $t \rightarrow V(T(t) x)$ is non-increasing for each $x \in X$;

(ii) If $x$ is such that $V(T(t) x)=V(x)$ for all $t \geq 0$, then $x \in \mathscr{E}$.

If $T(\cdot)$ is a gradient semigroup with an attractor $\mathscr{A}$ then given a global solution $\xi(\cdot)$ in $\mathscr{A}$, there are $x^{*}, y^{*} \in \mathscr{E}$ such that

$$
\lim _{t \rightarrow-\infty} \operatorname{dist}_{H}\left(\xi(t) x^{*}\right)=0 \quad \text { and } \quad \lim _{t \rightarrow \infty} \operatorname{dist}_{H}\left(\xi(t) y^{*}\right)=0 .
$$

Example 2.1.16. Consider the reaction-diffusion equation on a bounded domain $\Omega \subset \mathbb{R}^{n}$,

$$
\left\{\begin{array}{l}
u_{t}-\Delta u=f(u) \\
u=0, \quad \text { in } \partial \Omega .
\end{array}\right.
$$

If one imposes appropriated (dimension-dependent) growth condition on $f$, then this equation generates a semigroup on $H_{0}^{1}(\Omega)$ and the functional

$$
V(u)=\frac{1}{2}\|\nabla u\|_{L^{2}}^{2}-\int_{\Omega} F(u(x)) d x, \quad \text { with } \quad F(s)=\int_{0}^{s} f(r) d r
$$

is a Lyapunov function. 
Now we will see that for gradient systems we can completely describe the structure of its attractor: it is the unstable set of the equilibria.

Definition 2.1.17. If $x^{*} \in \mathscr{E}$, the unstable and stable manifold of $x^{*}$ is given respectively by $W^{u}\left(x^{*}\right)=\left\{y \in X:\right.$ there is a solution $\xi$ such that $\xi(0)=y$ and $\left.\operatorname{dist}\left(\xi(t), x^{*}\right) \stackrel{t \rightarrow-\infty}{\longrightarrow} 0\right\}$;

$$
W^{s}\left(x^{*}\right)=\left\{y \in X: \operatorname{dist}\left(T(t) y, x^{*}\right) \stackrel{t \rightarrow \infty}{\longrightarrow} 0\right\} .
$$

The local unstable and stable manifold of $x^{*}$ is given respectively by

$$
\begin{aligned}
& W_{\text {loc }}^{u}\left(x^{*}\right)=\{y \in X: \text { there is a solution } \xi \text { such that } \xi(0)=y \text { and } \\
& \left.\qquad \operatorname{dist}_{H}\left(\xi(t), x^{*}\right) \leq \delta, t \geq 0, \operatorname{dist}\left(\xi(t), x^{*}\right) \stackrel{t \rightarrow-\infty}{\longrightarrow} 0\right\} ; \\
& W_{\text {loc }}^{s}\left(x^{*}\right)=\left\{y \in X: \operatorname{dist}_{H}\left(T(t) y, x^{*}\right) \leq \delta, t \geq 0, \operatorname{dist}\left(T(t) y, x^{*}\right) \stackrel{t \rightarrow \infty}{\longrightarrow} 0\right\},
\end{aligned}
$$

for some $\delta>0$.

Theorem 2.1.18. If $T(\cdot)$ is a gradient semigroup with global attractor $\mathscr{A}$ and equilibrium set $\mathscr{E}=\left\{x_{1}^{*}, \ldots, x_{n}^{*}\right\}$, then

$$
\mathscr{A}=\bigcup_{i=1}^{n} W^{u}\left(x_{i}^{*}\right)
$$

Proof. If $x \in \mathscr{A}$, then there is a global solution $\varphi: \mathbb{R} \rightarrow X$ through $x$. Since $\varphi(\mathbb{R}) \subset \mathscr{A}$ and $\mathscr{A}$ is compact, the set

$$
\alpha_{\varphi}(x)=\left\{y \in X: \text { there is }\left\{t_{k}\right\}, t_{k} \stackrel{k \rightarrow \infty}{\longrightarrow}-\infty, \text { with } \lim _{k \rightarrow \infty} \varphi\left(t_{k}\right)=y\right\}
$$

is such that $\alpha_{\varphi}(x) \subset \mathscr{E}$ is not empty and $\operatorname{dist}_{H}\left(\varphi(t), \alpha_{\varphi}(x)\right) \stackrel{t \rightarrow-\infty}{\longrightarrow} 0$. It follows that $\mathscr{A} \subset W^{u}(\mathscr{E})$. To prove equality, note that if $x \in W^{u}(\mathscr{E})$, then there is a global solution $\varphi: \mathbb{R} \rightarrow X$ through $x$ and $\operatorname{dist}_{H}(\varphi(t), \mathscr{E}) \rightarrow 0$ as $t \rightarrow \pm \infty$. It follows that $\varphi(\mathbb{R})$ is bounded, and since it is also invariant, it follows that $\varphi(\mathbb{R}) \subset \mathscr{A}$ and $x=\varphi(0) \in \mathscr{A}$.

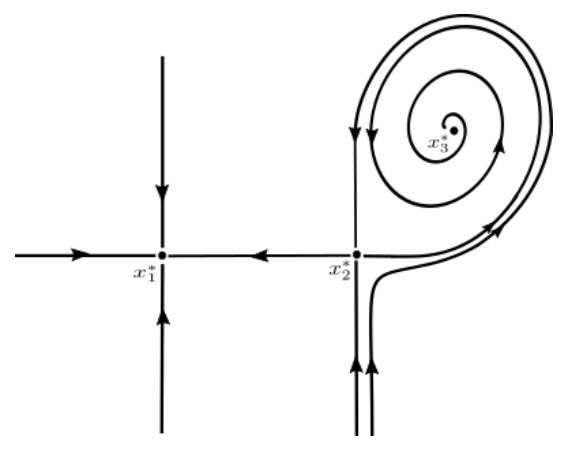

Figure 5 - Gadient Attractor

Example 2.1.19. In (HALE, 1988) it is possible to find an attractor given by the Figure 5. Note that we have $\mathscr{E}=\left\{x_{1}^{*}, x_{2}^{*}, x_{3}^{*}\right\}$ and the attractor is given by $\mathscr{A}=\bigcup_{i=1}^{3} W^{u}\left(x_{i}^{*}\right)$. 


\subsection{Semilinear Differential Equation}

Here we give a brief overview of some fundamental facts from theory of $C_{0}$-semigroup and abstract semilinear equation. Let $X$ a Banach space, we denote by $\mathscr{L}(X)$ the Banach space of the bounded linear operator from $X$ into itself.

Definition 2.2.1. A $C_{0}$-semigroup is a one parameter family $\{T(t): t \geq 0\}$ in $\mathscr{L}(X)$ such that

(i) $T(0)=I$,

(ii) $T(t+s)=T(t) T(s)$ for all $t, s \geq 0$,

(iii) $T(t) \stackrel{t \rightarrow 0^{+}}{\longrightarrow} x$ for all $x \in X$.

Definition 2.2.2. If $T(\cdot)$ is a $C_{0}$-semigroup we define its generator $A$ by

$$
\begin{aligned}
& D(A)=\left\{x \in X: \lim _{t \rightarrow 0^{+}} \frac{T(t) x-x}{t} \text { exists }\right\}, \\
& A x=\lim _{t \rightarrow 0^{+}} \frac{T(t) x-x}{t}, \quad \text { for all } x \in D(A) .
\end{aligned}
$$

It is natural to denote the $C_{0}$-semigroup whose generator is $A$ by $\left\{e^{A t}: t \geq 0\right\}$.

Example 2.2.3. Let $A$ be a bounded linear operator in Banach space $X$. Define

$$
e^{A t}=\sum_{n=0}^{\infty} \frac{A^{n} t^{n}}{n !} .
$$

The series converges absolutely since $\left\|A^{n}\right\|_{\mathscr{L}(X)} \leq\|A\|_{\mathscr{L}(X)}^{n}$. We have

$$
\left\|e^{A t}\right\|_{\mathscr{L}(X)} \leq \sum_{n=0}^{\infty} \frac{\left\|A^{n} t^{n}\right\|_{\mathscr{L}(X)}}{n !} \leq \sum_{n=0}^{\infty} \frac{\left(|t|\|A\|_{\mathscr{L}(X)}\right)^{n}}{n !}=e^{|t|\|A\|_{\mathscr{L}(X)}} .
$$

By multiplying the series we see

$$
e^{A(t+s)}=e^{A t} e^{A s}, \quad \text { for all } t, s \geq 0 .
$$

Also

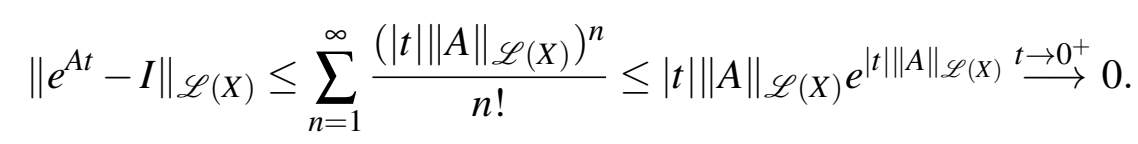

In many applications the space $X$ is a separable Hilbert space and we have the generator a self-adjoint invertible operator with

$$
\langle A u, u\rangle_{X} \geq \delta\|u\|_{X}^{2}, \quad \text { for some } \delta>0,
$$

and compact resolvent, that is, $(\lambda-A)^{-1}$ is compact for all $\lambda$ in resolvent set $\rho(A)$. Therefore the spectrum set is given by

$$
\sigma(A)=\left\{\lambda_{1}, \lambda_{2}, \lambda_{3}, \ldots\right\}
$$


where each $\lambda_{j}$ is an eigenvalue of $A$ with finite multiplicity $m_{j}$. It follows from (2.1) that $\lambda_{j} \geq \delta$, and we may assume without loss of generality that $\lambda_{j+1}>\lambda_{j}, j=1,2, \ldots$ If $M_{j}$ is the eigenspace associated with $\lambda_{j}$, then $\operatorname{dim}\left(M_{j}\right)=m_{j}, \mathrm{X}$ can be decomposed as the direct $\operatorname{sum} X=\oplus_{j=1}^{\infty} M_{j}$, where $M_{i} \perp M_{j}$ if $i \neq j$. If $P_{j}$ is the orthogonal projection onto $M_{j}$, then $A P_{j}=-\lambda_{j} P_{j}$ and

$$
A u=\sum_{j=1}^{\infty} \lambda_{j} P_{j} u
$$

In this context we can easily define the fractional powers of $A$ : for $\alpha \in \mathbb{R}, A^{\alpha}$ is given by

$$
A^{\alpha} u=\sum_{j=1}^{\infty} \lambda_{j}^{\alpha} P_{j} u, \quad \text { for all } \quad u \in D\left(A^{\alpha}\right),
$$

where

$$
D(A)=\left\{u \in X: \sum_{j=1}^{\infty} \lambda_{j}^{2 \alpha}\left\|P_{j} u\right\|_{X}^{2}<\infty\right\}
$$

for $\alpha>0$. Of course, $A^{\alpha}$ is a positive self-adjoint operator and, for $\alpha>0, D\left(A^{\alpha}\right)$ becomes a Hilbert space, denoted by $X^{\alpha}$, when equipped with the inner product $\langle u, v\rangle_{\alpha}=\left\langle A^{\alpha} u, A^{\alpha} v\right\rangle_{X}$, for which the corresponding norm is

$$
\|u\|_{\alpha}=\left\|A^{\alpha} u\right\|_{X}=\left(\sum_{j=1}^{\infty} \lambda_{j}^{2 \alpha}\left\|P_{j} u\right\|_{X}^{2}\right)^{\frac{1}{2}} .
$$

The $C_{0}$-semigroup whose generator is $-A$ is given by

$$
e^{-A t} u=\sum_{j=1}^{\infty} e^{-\lambda_{j} t} P_{j} u
$$

and this semigroup smooths, in the sense that for $t>0$ we have $e^{A t} u_{0} \in X^{\alpha}$ when $u_{0} \in X$. We have the following inequality

$$
\left\|e^{-A t}\right\|_{\mathscr{L}\left(X^{\beta}, X^{\alpha}\right)} \leq C t^{\beta-\alpha}, \text { for all } \quad \alpha>\beta .
$$

It is known that $e^{-A t}$ is also obtained by

$$
e^{-A t}=\frac{1}{2 \pi i} \int_{\Gamma} e^{\lambda t}(\lambda+A)^{-1} d \lambda, \text { for all } t>0,
$$

where $\Gamma$ is the curve in the complex plane given by the boundary of

$$
\{\lambda \in \mathbb{C}:|\arg (\lambda)| \geq \varphi\} \backslash\{\lambda \in \mathbb{C}:|\lambda|<r\}
$$

oriented in such away that the imaginary part is increasing. Also note that

$$
(\lambda+A)^{-1}=\sum_{j=1}^{\infty} \frac{1}{\lambda-\lambda_{j}} P_{j}
$$

and hence for $\lambda \in \sigma(A)$

$$
\left\|(\lambda+A)^{-1}\right\|_{\mathscr{L}(X)} \leq \frac{1}{\operatorname{dist}(\lambda, \sigma(A))} .
$$

In particular $A$ is a sectorial operator matching with the following definition. 
Definition 2.2.4. We say that a closed and densely defined linear operator $A: D(A) \subset X \rightarrow X$ is a positive sectorial operator if, for some $\varphi \in(0, \pi / 2)$ and $M>0$,

$$
\Sigma_{\varphi}:=\{\lambda \in \mathbb{C}|\arg (\lambda)| \geq \varphi\} \cup\{0\} \subset \rho(-A)
$$

and

$$
\left\|(\lambda+A)^{-1}\right\|_{\mathscr{L}(X)} \leq \frac{M}{1+|\lambda|}, \quad \text { for all } \lambda \in \Sigma_{\varphi}
$$

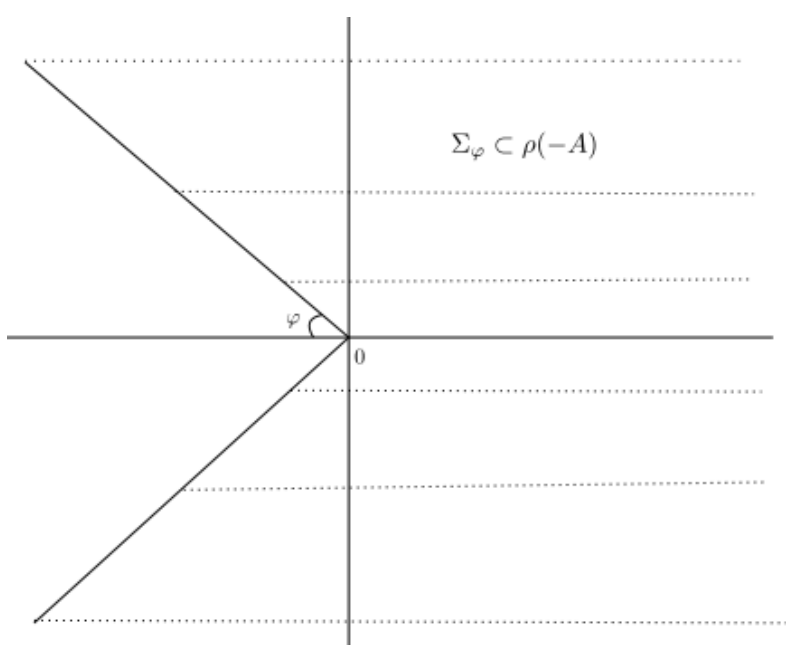

Figure 6 - Sector

The main result about definition 2.2.4 is that when $A$ is a sectorial operator, the linear semigroup $e^{-A t}$ is an analytic semigroup given by expression (2.2).

Example 2.2.5. Consider the reaction-diffusion equation

$$
\left\{\begin{array}{l}
u_{t}-\Delta u=f(u), x \in \Omega \\
\left.u\right|_{\partial \Omega}=0
\end{array}\right.
$$

where $\Omega$ is a smooth domain in $\mathbb{R}^{N}$. If we define $A=-\Delta$ and $D(A)=\left\{u \in H^{2}(\Omega): u=0\right.$ on $\left.\partial \Omega\right\}$. With this choice of $D(A)$, the operator $A$ is self adjoint and has compact resolvent. Therefore $A$ is a sectorial operator. It is well known that $X^{\frac{1}{2}}=H_{0}^{1}(\Omega)$, see (HENRY, 1980).

We are concerning in treat semilinear parabolic problems of the form

$$
\left\{\begin{array}{l}
\dot{u}+A u=f(u) \\
u\left(t_{0}\right)=u_{0} \in X
\end{array}\right.
$$

where $A$ is a sectorial operator and we assume, without loss of generality, that $\operatorname{Re}(\sigma(A))>\delta$ for some $\delta>0$. We assume that $f: X^{1} \rightarrow X^{\alpha}$ is continuously differentiable and locally bounded. 
Therefore (2.4) is globally well posed in $X^{\alpha}$, that is, there is a continuous function $u:\left[t_{0}, \infty\right) \rightarrow X$ which is differentiable in $\left(t_{0}, \infty\right)$ and such that $u(t) \in D(A)$ for all $t>t_{0}, t \rightarrow A u(t)$ is continuous for all $t>t_{0}$ and (2.4) is satisfied. Moreover $u$ verify the variation of constant formula

$$
u(t)=e^{-A\left(t-t_{0}\right)} u_{0}+\int_{t_{0}}^{t} e^{-A(t-s)} f(u(s)) d s, \quad t>t_{0} .
$$

If we assume some dissipative conditions in $f$ we obtain that the semigroup $T(\cdot)$ given by the solution of (2.4) has a global attractor $\mathscr{A}$. It is well known that the attractor can be understanding as a finite dimension object. In fact we will consider in the next chapters problems whose the attractor lying in a finite dimensional invariant manifold.

Definition 2.2.6. A set $\mathscr{M} \subset X$ is an invariant manifold for (2.4) when for each $u_{0} \in \mathscr{M}$ there is a global solution $u(\cdot)$ such that $u(0)=u_{0}$ and $u(t) \in \mathscr{M}$ for all $t \in \mathbb{R}$.

Example 2.2.7. Consider the problem (2.3) in scalar form

$$
\left\{\begin{array}{l}
u_{t}-u_{x x}=f(u), 0<x<1, \\
u=0, \text { in }\{0,1\} .
\end{array}\right.
$$

We assume the appropriated conditions for that (2.5) generate a semigroup in $X^{\frac{1}{2}}$ with a global attractor $\mathscr{A}$. The eigenvalues of $A u=u_{x x}$ has the property known as gap condition. With this property we can construct a finite invariant manifold $\mathscr{M}$ for (2.5) such that $\mathscr{A} \subset \mathscr{M}$. For details see (HENRY, 1985).

Now we study in detail a divergent form operator that we will use in the next chapters. Let $\varepsilon \in(0,1)$ and $p_{\varepsilon} \in C^{1}[0,1]$. Define $A_{\varepsilon}: D\left(A_{\varepsilon}\right) \subset L^{2}(0,1) \rightarrow L^{2}(0,1)$, by

$$
\begin{gathered}
A_{\varepsilon} u=-\left(p_{\varepsilon}(x) u_{x}\right)_{x}+(\lambda+c(x)) u, \\
D\left(A_{\varepsilon}\right)=\left\{u \in H^{2}(\Omega): u_{x}(0)=0=u_{x}(1)\right\},
\end{gathered}
$$

where $c \in C^{1}([0,1])$ and $\lambda \in \mathbb{R}$ is such that

$$
0<m_{0} \leq \min _{x \in[0,1]} c(x)+\lambda
$$

for some positive constant $m_{0}$.

We have the following result.

Proposition 2.2.8. The operator $A_{\varepsilon}$ is positive, self-adjoint, has compact resolvent and $0 \in$ $\rho\left(A_{\varepsilon}\right)$.

Proof. Define the following bilinear form

$$
a_{\varepsilon}(u, v)=\int_{\Omega} p_{\varepsilon}(x) u_{x} v_{x} d x+\int_{\Omega} \bar{\lambda} u v d x, \quad \forall u, v \in H^{1}(\Omega),
$$


where we denote $\bar{\lambda}=\lambda+c$ and $\Omega=(0,1)$.

We claim that $a_{\varepsilon}$ is coercive and continuous. In fact, for $u \in H^{1}(\Omega)$, we have

$$
\begin{aligned}
a_{\mathcal{\varepsilon}}(u, u) & =\int_{\Omega} p_{\varepsilon}(x) u_{x} u_{x} d x+\int_{\Omega} \bar{\lambda} u u d x \\
& =\int_{\Omega} p_{\varepsilon}(x)\left|u_{x}\right|^{2} d x+\int_{\Omega} \bar{\lambda}|u|^{2} d x \\
& \geq m_{0} \int_{\Omega}\left|u_{x}\right|^{2} d x+\int_{\Omega} \bar{\lambda}|u|^{2} d x \\
& \geq m_{0}\left(\int_{\Omega}\left|u_{x}\right|^{2} d x+\int_{\Omega}|u|^{2} d x\right) \\
& =m_{0}\|u\|_{H^{1}(\Omega)}^{2} .
\end{aligned}
$$

and for $u, v \in H^{1}(\Omega)$,

$$
\begin{aligned}
a_{\varepsilon}(u, v) & =\int_{\Omega} p_{\varepsilon}(x) u_{x} v_{x} d x+\int_{\Omega} \bar{\lambda} u v d x \\
& \leq M_{\varepsilon}\left\|u_{x}\right\|_{L^{2}(\Omega)}\left\|v_{x}\right\|_{L^{2}(\Omega)}+M_{\bar{\lambda}}\|u\|_{L^{2}(\Omega)}\|v\|_{L^{2}(\Omega)} \\
& \leq \max \left\{M_{\mathcal{E}}, M_{\bar{\lambda}}\right\}\|u\|_{H^{1}(\Omega)}\|v\|_{H^{1}(\Omega)},
\end{aligned}
$$

where $M_{\varepsilon}=\sup _{x \in[0,1]} p_{\varepsilon}(x)$ and $M_{\bar{\lambda}}=\sup _{x \in[0,1]} \bar{\lambda}(x)$.

Now consider the operator $B_{\varepsilon}: H^{1}(\Omega) \rightarrow H^{-1}(\Omega)$, given by the relation

$$
a_{\varepsilon}(u, v)=\left\langle B_{\varepsilon} u, v\right\rangle_{-1,1},
$$

where $\langle\cdot, \cdot\rangle_{-1,1}$ denotes the inner product between the duality $H^{1}(\Omega)$ and $\left(H^{1}(\Omega)\right)^{*}:=H^{-1}(\Omega)$. Hence $B_{\varepsilon}$ is continuous and injective, since $\left\langle B_{\varepsilon} u, u\right\rangle_{-1,1}=0$ impies $u=0$ and, by Lax-Milgram Theorem, for any $\varphi \in H^{-1}(\Omega)$, there is a unique $u \in H^{1}(\Omega)$ such that

$$
\left\langle B_{\varepsilon} u, v\right\rangle_{-1,1}=a_{\varepsilon}(u, v)=\langle\varphi, v\rangle_{-1,1}, \quad \forall v \in H^{1}(\Omega),
$$

that is, $B_{\varepsilon} u=\varphi$, hence $B_{\varepsilon}$ is also surjective. Thus $B_{\varepsilon}$ is an isomorphism and we can consider the operator $\tilde{A}_{\varepsilon}: D\left(\tilde{A}_{\varepsilon}\right) \subset L^{2}(\Omega) \rightarrow L^{2}(\Omega)$ defined by

$$
\begin{gathered}
D\left(\tilde{A}_{\varepsilon}\right)=\left\{u \in H^{1}(\Omega) ; B_{\varepsilon} u \in L^{2}(\Omega) \text { e } u_{x}=0 \text { in } \partial \Omega\right\} ; \\
\tilde{A}_{\varepsilon} u=-\left(p_{\varepsilon}(x) u_{x}\right)_{x}+\bar{\lambda} u, \quad \forall u \in D\left(\tilde{A}_{\varepsilon}\right) .
\end{gathered}
$$

We have $D\left(\tilde{A}_{\varepsilon}\right)=\left\{u \in H^{2}(\Omega) ; u_{x}=0\right.$ in $\left.\partial \Omega\right\}$. Let $u \in D\left(\tilde{A}_{\varepsilon}\right)$, then $B_{\varepsilon} u \in L^{2}(\Omega)$ and for all $\varphi \in H^{1}(\Omega)$

$$
\int_{\Omega} p_{\varepsilon}(x) u_{x} \varphi_{x} d x+\int_{\Omega} \bar{\lambda} u \varphi d x=\int_{\Omega} B_{\varepsilon} u \varphi d x,
$$

that is, $u$ is a weak solution of

$$
\left\{\begin{array}{l}
-\left(p_{\varepsilon}(x) u\right)_{x}+\bar{\lambda} u=B_{\varepsilon} u \text { on } \Omega \\
u_{x}=0 \text { in } \partial \Omega
\end{array} .\right.
$$


hence $u \in H^{2}(\Omega)$ and $u_{x}=0$ on $\partial \Omega$. On the other hand, if $u \in H^{2}(\Omega)$ and $u_{x}=0$ on $\partial \Omega$, then $u \in H^{1}(\Omega)$ and $B_{\varepsilon} u \in H^{1}(\Omega) \hookrightarrow L^{2}(\Omega)$. We can write

$$
\begin{aligned}
\left\langle B_{\varepsilon} u, v\right\rangle_{-1,1} & =\int_{\Omega} p_{\varepsilon}(x) u_{x} v_{x} d x+\int_{\Omega} \bar{\lambda} u v d x \\
& =\int_{\Omega}-\left(p_{\varepsilon}(x) u_{x}\right)_{x} v d x+\int_{\Omega} \bar{\lambda} u v d x \\
& =\left\langle-\left(p_{\varepsilon}(x) u_{x}\right)_{x}+\bar{\lambda} u, v\right\rangle_{L^{2}(\Omega)} \\
& =\left\langle\tilde{A}_{\varepsilon} u, v\right\rangle_{L^{2}(\Omega)} .
\end{aligned}
$$

Since $u \in L^{2}(\Omega)$ it follows that $B_{\varepsilon} u \in L^{2}(\Omega) \Leftrightarrow-\left(p_{\varepsilon}(x) u_{x}\right)_{x} \in L^{2}(\Omega)$. Hence

$$
D\left(\tilde{A}_{\varepsilon}\right)=\left\{u \in H^{1}(\Omega) ;-\left(p_{\varepsilon}(x) u_{x}\right)_{x} \in L^{2}(\Omega) \text { and } u_{x}=0 \text { on } \partial \Omega\right\}=D\left(A_{\varepsilon}\right)
$$

and $\tilde{A}_{\varepsilon}=A_{\varepsilon}$. Thus $a_{\varepsilon}(u, v)=\left\langle A_{\varepsilon} u, v\right\rangle_{L^{2}(\Omega)}$ wich implies $A_{\varepsilon}$ positive and simmetric. Since $A_{\varepsilon}$ is surjective, we obtain $A_{\varepsilon}$ self-adjoint. Since $A_{\varepsilon}$ is an isomorphism and closed, we have $A_{\varepsilon}^{-1}$ is closed wich implies $A_{\varepsilon}^{-1} \in \mathscr{L}\left(L^{2}(\Omega)\right)$, that is $0 \in \rho\left(A_{\varepsilon}\right)$. In order to prove that $A_{\varepsilon}^{-1}$ is compact, let $\left\{g_{\varepsilon}\right\}_{\varepsilon} \subset L^{2}(\Omega)$ such that $\left\|g_{\varepsilon}\right\|_{L^{2}} \leq 1$. For $v^{\varepsilon}=A_{\varepsilon}^{-1} g_{\varepsilon}$, we have

$$
\int_{\Omega} p_{\varepsilon}(x)\left|v_{x}^{\varepsilon}\right|^{2} d x+\int_{\Omega} \bar{\lambda}\left|v^{\varepsilon}\right|^{2} d x=a_{\varepsilon}\left(v^{\varepsilon}, v^{\varepsilon}\right)=\left\langle A_{\varepsilon} v^{\varepsilon}, v^{\varepsilon}\right\rangle_{L^{2}(\Omega)}=\int_{\Omega} g_{\varepsilon} v^{\varepsilon} d x .
$$

Taking $C_{1}$ as constant immersion of $H^{1}(\Omega)$ in $L^{2}(\Omega)$ and $0<\delta<\frac{2 m_{0}}{C_{1}^{2}}$, it follows by Young's inequality ${ }^{1}$ that

$$
\begin{aligned}
m_{0}\left\|v^{\varepsilon}\right\|_{H^{1}(\Omega)}^{2} & \leq \int_{\Omega} p_{\varepsilon}(x)\left|v_{x}^{\varepsilon}\right|^{2} d x+\int_{\Omega} \bar{\lambda}\left|v^{\varepsilon}\right|^{2} d x \\
& \leq \int_{\Omega}\left|g_{\varepsilon} \| v^{\varepsilon}\right| d x \leq \int_{\Omega} \frac{\left|g_{\varepsilon}\right|^{2}}{2 \delta}+\frac{\delta\left|v^{\varepsilon}\right|^{2}}{2} d x \\
& =\frac{\left\|g_{\varepsilon}\right\|_{L^{2}(\Omega)}^{2}}{2 \delta}+\frac{\delta\left\|v^{\varepsilon}\right\|_{L^{2}(\Omega)}^{2}}{2} \\
& \leq \frac{1}{2 \delta}+\frac{\delta C_{2}^{2}}{2}\left\|v^{\varepsilon}\right\|_{H^{1}(\Omega)}^{2},
\end{aligned}
$$

hence

$$
\left\|v^{\varepsilon}\right\|_{H^{1}(\Omega)}^{2} \leq C_{3}, \quad \text { where } C_{3}=\frac{1}{2 \delta}\left(C_{1}-\frac{\delta C_{2}^{2}}{2}\right)^{-1}>0 .
$$

Therefore, there is $M>0$ such that

$$
A_{\varepsilon}^{-1}\left(B_{L^{2}(\Omega)}(0,1)\right) \subset B_{H^{1}(\Omega)}(0, M)
$$

Since $H^{1}(\Omega) \hookrightarrow L^{2}(\Omega)$ compactly, we have $A_{\mathcal{\varepsilon}}^{-1}$ compact.

$1 \quad a, b \geq 0, \forall \delta>0 \Rightarrow a b \leq \frac{a^{2}}{2 \delta}+\frac{\delta b^{2}}{2}$. 


\subsection{Rate of Convergence of Attractors}

In this section we study the rate of continuity (or convergence or attraction) of attractors under perturbation. We consider a family of semigroups $\left\{T_{\varepsilon}(\cdot)\right\}_{\varepsilon \in \Lambda}$, where $\varepsilon$ is a parameter in a metric space $\Lambda$. We will assume that the semigroup converges (in some appropriated sense) to $T_{0}(\cdot)$ as $\varepsilon \rightarrow \varepsilon_{0}$.

Definition 2.3.1. We say that a family $\left\{\mathscr{A}_{\varepsilon}\right\}_{\varepsilon \in \Lambda}$ of subsets of $X$ is continuous at $\varepsilon_{0}$ if

$$
\mathrm{d}_{H}\left(\mathscr{A}_{\varepsilon}, \mathscr{A}_{0}\right) \stackrel{\varepsilon \rightarrow \varepsilon_{0}}{\longrightarrow} 0 .
$$

Definition 2.3.2. If $T_{\mathcal{E}}(\cdot)$ is a family of semigroups with attractors $\mathscr{A}_{\mathcal{E}}$, then we say that $\left\{\mathscr{A}_{\varepsilon}\right\}_{\varepsilon \in \Lambda}$ is equi-attracting if

$$
\sup _{\varepsilon \in \Lambda} \operatorname{dist}_{H}\left(T_{\varepsilon}(t) B, \mathscr{A}_{\varepsilon}\right) \stackrel{t \rightarrow \infty}{\longrightarrow} 0
$$

for each bounded subset $B$ of $X$.

We now see that equi-attraction implies the continuity of attractors, we can also obtain a rate of convergence of attractors with respect to the underlying parameter.

Theorem 2.3.3. Let $T_{\varepsilon}(\cdot)$ be a family of semigoups with attractors $\mathscr{A}_{\varepsilon}$ and set $D=\cup_{\varepsilon \in \Lambda} \mathscr{A}_{\varepsilon}$. If there is a strictly decreasing function $\eta:[0, \infty) \rightarrow(0, \infty)$ such that

$$
\sup _{\varepsilon \in \Lambda} \operatorname{dist}_{H}\left(T_{\varepsilon}(t) D, \mathscr{A}_{\varepsilon}\right) \leq \eta(t), \quad t \geq 0
$$

and, there are $C, L>0$ and a function $\tau(\varepsilon) \rightarrow 0$ as $\varepsilon \rightarrow \varepsilon_{0}$ such that

$$
\operatorname{dist}_{H}\left(T_{\varepsilon}(t) x, T_{0}(t) y\right) \leq C e^{L t}\left(\|x-y\|_{X}+\tau(\varepsilon)\right), \quad t \geq 0 .
$$

Then

$$
\left.\mathrm{d}_{H}\left(\mathscr{A}_{\varepsilon}, \mathscr{A}_{0}\right) \leq \min _{v \in \eta([0, \infty))} 2\left\{C e^{L \eta^{-1}(v)} \tau(\varepsilon)+v\right)\right\}
$$

Proof. For $t \geq 0$ we have

$$
\begin{aligned}
\operatorname{dist}_{H}\left(\mathscr{A}_{\varepsilon}, \mathscr{A}_{0}\right) & \leq \operatorname{dist}_{H}\left(T_{\varepsilon}(t) \mathscr{A}_{\varepsilon}, T_{0}(t) \mathscr{A}_{\varepsilon}\right)+\operatorname{dist}_{H}\left(T_{0}(t) \mathscr{A}_{\varepsilon}, \mathscr{A}_{0}\right) \\
& \leq \sup _{x \in \mathscr{A}_{\varepsilon}} \operatorname{dist}_{H}\left(T_{\varepsilon}(t) x, T_{0}(t) x\right)+\operatorname{dist}_{H}\left(T_{0}(t) \mathscr{A}_{\varepsilon}, \mathscr{A}_{0}\right) \\
& \leq C e^{L t} \tau(\varepsilon)+\eta(t) .
\end{aligned}
$$

Given $v \in \eta([0, \infty))$ e $t=\eta^{-1}(v)$,

$$
\operatorname{dist}_{H}\left(\mathscr{A}_{\varepsilon}, \mathscr{A}_{0}\right) \leq C e^{L \eta^{-1}(v)} \tau(\varepsilon)+v .
$$


In particular when the equi-attraction is exponential, we can give a precise rate of convergence.

Corollary 2.3.4. Assume that the conditions of Theorem 2.3 .3 are satisfied with $\eta(t)=c e^{-\gamma t}$ for some $c \geq 1, \gamma>0$ and for all $t \in[0, \infty)$. Then there is a constant $\bar{c}>0$ independent of $\varepsilon$, such that

$$
\mathrm{d}_{H}\left(\mathscr{A}_{\varepsilon}, \mathscr{A}_{0}\right) \leq \bar{c} \tau(\varepsilon)^{\frac{\gamma}{\gamma+L}}
$$

Proof. Take $\eta^{-1}(v)=\log \left(\frac{c}{v}\right)^{\frac{1}{\gamma}}$, we obtain

$$
\mathrm{d}_{H}\left(\mathscr{A}_{\varepsilon}, \mathscr{A}_{0}\right) \leq 2 \min _{v \in(0, c]}\left[\tau(\varepsilon)\left(\frac{c}{v}\right)^{\frac{L}{\gamma}}+v\right] .
$$

The minimum of the right-hand side of (2.6) occurs when $v=c\left(\frac{L}{\gamma}\right)^{\frac{\gamma}{\gamma+L}} \tau(\varepsilon)^{\frac{\gamma}{\gamma+L}}$. Since the lefthand size of (2.6) is independent of $v$, it follows that

$$
\mathrm{d}_{H}\left(\mathscr{A}_{\varepsilon}, \mathscr{A}_{0}\right) \leq 2 c\left[\left(\frac{L}{\gamma}\right)^{\frac{-L}{\gamma+L}}+\left(\frac{L}{\gamma}\right)^{\frac{\gamma}{\gamma+L}}\right] \tau(\varepsilon)^{\frac{\gamma}{\gamma+L}} .
$$

Example 2.3.5. Consider the Cauchy problem for the following second order ordinary differential equation

$$
\left\{\begin{array}{l}
\varepsilon \ddot{x}+\dot{x}=\mu x+f(x), \quad x \in \mathbb{R}^{N} \\
x(0)=x_{0} \in \mathbb{R}^{N} \\
x_{t}(0)=v_{0} \in \mathbb{R}^{N} .
\end{array}\right.
$$

Assume that $\varepsilon \in[0,1], \mu \geq 1, f: \mathbb{R}^{N} \rightarrow \mathbb{R}^{N}$ is a $C^{1}$ function which is globally Lipschitz, globally bounded and with symmetric Jacobian matrix at every point. If we write the above equation in the form of a system with variables $x$ and $v=\varepsilon \dot{x}$ we have that

$$
\left\{\begin{array}{l}
\frac{d}{d t}\left[\begin{array}{l}
x \\
v
\end{array}\right]=\left[\begin{array}{cc}
0 & I / \varepsilon \\
-\mu & -I / \varepsilon
\end{array}\right]\left[\begin{array}{l}
x \\
v
\end{array}\right]+\left[\begin{array}{c}
0 \\
f(x)
\end{array}\right],\left[\begin{array}{l}
x \\
v
\end{array}\right] \in \mathbb{R}^{N} \times \mathbb{R}^{N} \\
{\left[\begin{array}{l}
x \\
v
\end{array}\right](0)=\left[\begin{array}{c}
x_{0} \\
\varepsilon v_{0}
\end{array}\right] \in \mathbb{R}^{N} \times \mathbb{R}^{N}}
\end{array}\right.
$$

Clearly, the solutions are globally defined and the solution operator family $T_{\mathcal{\varepsilon}}(\cdot)$ defines a semigroup in $Z=\mathbb{R}^{N} \times \mathbb{R}^{N}$. Furthermore $T_{\mathcal{\varepsilon}}(\cdot)$ has a global attractor $\mathscr{A}_{\mathcal{\varepsilon}}$. The above system can be rewritten as

$$
\left\{\begin{array}{l}
\frac{d}{d t}\left[\begin{array}{cr}
I & I \\
-\varepsilon \mu I & 0
\end{array}\right]\left[\begin{array}{l}
x \\
v
\end{array}\right]=-\left[\begin{array}{l}
x \\
v
\end{array}\right]+\left[\begin{array}{cc}
I & I \\
-\varepsilon \mu I & 0
\end{array}\right]\left[\begin{array}{c}
0 \\
f(x)
\end{array}\right],\left[\begin{array}{l}
x \\
v
\end{array}\right] \in \mathbb{R}^{N} \times \mathbb{R}^{N} \\
{\left[\begin{array}{l}
x \\
v
\end{array}\right](0)=\left[\begin{array}{l}
x_{0} \\
\varepsilon v_{0}
\end{array}\right] \in \mathbb{R}^{N} \times \mathbb{R}^{N} .}
\end{array}\right.
$$


As the parameter $\varepsilon$ tends to zero, one would expect that the dynamical properties of (2.7) are given by

$$
\left\{\begin{array}{l}
\frac{d}{d t}\left[\begin{array}{ll}
I & I \\
0 & 0
\end{array}\right]\left[\begin{array}{l}
x \\
v
\end{array}\right]=-\left[\begin{array}{l}
x \\
v
\end{array}\right]+\left[\begin{array}{cc}
I & I \\
0 & 0
\end{array}\right]\left[\begin{array}{c}
0 \\
f(x)
\end{array}\right], \quad\left[\begin{array}{l}
x \\
v
\end{array}\right] \in \mathbb{R}^{N} \times \mathbb{R}^{N} \\
{\left[\begin{array}{l}
x \\
v
\end{array}\right](0)=\left[\begin{array}{l}
x_{0} \\
\varepsilon v_{0}
\end{array}\right] \in \mathbb{R}^{N} \times \mathbb{R}^{N}}
\end{array}\right.
$$

which corresponds to $v=0$ and

$$
\left\{\begin{array}{l}
\dot{x}=-\mu x+f(x) \\
x(0)=x_{0} \in \mathbb{R}^{N}
\end{array}\right.
$$

Note that the solutions for (2.8) are globally defined and the solution operator family $R_{0}(\cdot)$ defines a semigroup in $\mathbb{R}^{N}$. To compare the dynamics of these two problems we should find a way to see the dynamics of (2.8) in $Z$. That is done simply defining

$$
T_{0}(t)\left[\begin{array}{l}
x_{0} \\
v_{0}
\end{array}\right]=\left[\begin{array}{c}
R_{0}(t) x_{0} \\
0
\end{array}\right], \quad t>0, \quad T_{0}(0)=I,
$$

and noting that $T_{0}(\cdot)$ is a semigroup (singular at zero) having a global attractor $\mathscr{A}_{0}$. We can prove (see (CARVALHO; CHOLEWA; T.DLOTKO, 2010)) that there are constants $\varepsilon_{0}>0, \gamma>0$, $t_{0}>0$ and a constant $C>0$ independent of $\varepsilon$ such that

$$
\operatorname{dist}_{H}\left(T_{\mathcal{E}}(t) \bigcup_{\varepsilon \in\left[0, \varepsilon_{0}\right]} \mathscr{A}_{\mathcal{\varepsilon}}, \mathscr{A}_{\varepsilon}\right) \leq C e^{-\gamma t}, \quad t \geq t_{0}
$$

and for $\bar{x}, \bar{y} \in Z$,

$$
\left\|T_{\mathcal{E}}(t) \bar{x}-T_{0}(t) \bar{y}\right\|_{Z} \leq C e^{L t},\|\bar{x}-\bar{y}\|_{Z}+\varepsilon^{\alpha}, \quad t \geq t_{0}, \quad \alpha<1 .
$$

Then by Corollary 2.3.4

$$
\mathrm{d}_{H}\left(\mathscr{A}_{\varepsilon}, \mathscr{A}_{0}\right) \leq C \varepsilon^{\frac{\alpha \gamma}{\gamma+L}}
$$

Example 2.3.6. Consider the semilinear parabolic problems of the form

$$
\left\{\begin{array}{l}
u_{t}^{\varepsilon}-\operatorname{div}\left(p_{\varepsilon}(x) \nabla u^{\varepsilon}\right)=f\left(u^{\varepsilon}\right), x \in \Omega, t>0 \\
u^{\varepsilon}(t, x)=0, x \in \partial \Omega, t>0 \\
u^{\varepsilon}(0, x)=u_{0}^{\varepsilon}(x)
\end{array}\right.
$$

where $\Omega \subset \mathbb{R}^{N}, N \geq 2$, is a bounded smooth domain, $\varepsilon \in[0,1]$ is a parameter, $p_{\varepsilon} \in C^{1}([0,1])$ and $f: \mathbb{R} \rightarrow \mathbb{R}$ is continuously differentiable such that

$$
\limsup _{|s| \rightarrow \infty} \frac{f(s)}{s} \leq 0
$$


We can write (2.9) as

$$
\left\{\begin{array}{l}
u_{t}^{\varepsilon}+A_{\varepsilon} u^{\varepsilon}=f(u), t>0 \\
u^{\varepsilon}(0)=u_{0}^{\varepsilon},
\end{array}\right.
$$

where $f$ denotes the Nemitskii functional and

$$
\begin{gathered}
A_{\varepsilon} u=-\operatorname{div}\left(p_{\varepsilon} \nabla u\right), \\
D\left(A_{\varepsilon}\right)=\left\{u \in H^{1}(\Omega):-\operatorname{div}\left(p_{\varepsilon} \nabla u\right) \in L^{2}(\Omega), u=0 \text { on } \partial \Omega\right\} .
\end{gathered}
$$

Assuming appropriated growth conditions the problem (2.10) is well posed in $H_{0}^{1}(\Omega)$, that is, the solutions generate a semigroup $T_{\varepsilon}(\cdot)$ with a global attractor $\mathscr{A}_{\varepsilon}$ for all $\varepsilon \in[0,1]$. In (ARRIETA; BEZERRA; CARVALHO, 2013), the authors assumed that

$$
\left\|p_{\varepsilon}-p_{0}\right\|_{L^{\infty}} \stackrel{\varepsilon \rightarrow 0}{\longrightarrow} 0
$$

and obtained that for $u^{\varepsilon}, u^{0} \in H_{0}^{1}(\Omega)$,

$$
\left\|T_{\varepsilon}(t) u^{\varepsilon}-T_{0}(t) u^{0}\right\|_{H_{0}^{1}} \leq C e^{L t} t^{-\frac{1}{2}-\theta}\left(\left\|u^{\varepsilon}-u^{0}\right\|_{H_{0}^{1}}+\left\|p_{\varepsilon}-p_{0}\right\|_{L^{\infty}}^{2 \theta}\right), \quad 0<\theta<\frac{1}{2}, t \geq 0,
$$

and

$$
\operatorname{dist}_{H}\left(T_{\mathcal{E}}(t) \bigcup_{\varepsilon \in\left[0, \varepsilon_{0}\right]} \mathscr{A}_{\mathcal{E}}, \mathscr{A}_{\varepsilon}\right) \leq C e^{-\gamma t}, \quad t \geq 0 .
$$

Then by Corollary 2.3.4

$$
\mathrm{d}_{H}\left(\mathscr{A}_{\varepsilon}, \mathscr{A}_{0}\right) \leq C\left\|p_{\varepsilon}-p_{0}\right\|_{L^{\infty}}^{\frac{2 \theta \gamma}{\gamma+L}}
$$

where $C$ is a constant independent of $\varepsilon$.

\subsection{Morse-Smale Map}

In this section we treat the Morse-Smale semigroups. The results stated here can be found in (BORTOLAN et al., 2015).

We start defining the non-wandering set for a semigroup.

Definition 2.4.1. We define the non-wandering set $W$ of a semigroup $T(\cdot)$ with attractor $\mathscr{A}$ as the set of points $x \in \mathscr{A}$ such that given $t_{0} \geq 0$ and a neigborhood $V_{x}$ of $x$ in $X$, there is $t \geq t_{0}$ such that $T(t) V_{x} \cap V_{x} \neq \emptyset$.

In order to define hyperbolic point we consider continuously differentiable semigroups, that is, $T(t): X \rightarrow X$ is differentiable on each $x \in X$ and $[0, \infty) \times X \ni(t, x) \rightarrow D_{x} T(t) \in \mathscr{L}(X)$ is continuous.

In what follows we consider $T(\cdot)$ a continuously differentiable semigroup. 
Definition 2.4.2. An equilibrium point $x^{*}$ of $T(\cdot)$ is said to be hyperbolic if

$$
\sigma\left(\left.D_{x} T(t)\right|_{x=x^{*}}\right) \cap\{\lambda \in \mathbb{C}:|\lambda|=1\}=\emptyset, \quad t>0 .
$$

Now we are ready to define Morse-Smale semigroup.

Definition 2.4.3. We say that the semigroup $T(\cdot)$ with attractor $\mathscr{A}$ is a Morse-Smale semigroup if it satisfies the following conditions:

(i) $T(\cdot)$ is reversible; that is, $\left.T(t)\right|_{\mathscr{A}}$ is injective, $T(t)$ is differentiable in $\mathscr{A}$ and $T^{\prime}(t)(z)$ : $X \rightarrow X$ is an injective bounded linear operator, for all $t \geq 0$ and $z \in \mathscr{A}$.

(ii) there is a finite number of hyperbolic equilibrium points $\mathscr{E}=\left\{x_{1}^{*}, \ldots, x_{n}^{*}\right\}$ such that $\mathscr{E}=W$.

(iii) if $x^{*} \in \Omega$ then $\operatorname{dim}\left(W_{\mathrm{loc}}^{u}\left(x^{*}\right)\right)<\infty$.

(iv) If $x_{1}^{*}, x_{2}^{*} \in \mathscr{E}$ then $W^{u}\left(x_{1}^{*}\right)$ and $W_{\text {loc }}^{s}\left(x_{2}^{*}\right)$ intersect transversally; that is, if $z \in W^{u}\left(x_{1}^{*}\right) \cap$ $W_{\text {loc }}^{s}\left(x_{2}^{*}\right)$ then $T_{z} W^{u}\left(x_{1}^{*}\right)+T_{z} W_{\text {loc }}^{s}\left(x_{2}^{*}\right)=X$, where $T_{z} W^{u}\left(x_{1}^{*}\right), T_{z} W_{\text {loc }}^{s}\left(x_{2}^{*}\right)$ are the tangent spaces of $W^{u}\left(x_{1}^{*}\right), W_{\text {loc }}^{s}\left(x_{2}^{*}\right)$, respectively in $X$ at point $z$.

Example 2.4.4. Consider the scalar equation

$$
\left\{\begin{array}{l}
u_{t}=u_{x x}+f\left(x, u, u_{x}\right), 0<x<1, \\
u=0, \text { in }\{0,1\} .
\end{array}\right.
$$

In (HENRY, 1985) is proved that the equation (2.12) has the property (iv) in the Definition 2.4.3. Moreover with some appropriated conditions we can obtain the others statements. Hence the semigroup generated by (2.12) is Morse-Smale.

The main property about Morse-Smale semigroup is the structural stability state in the next theorem.

Theorem 2.4.5. Let $\left\{T_{\varepsilon}(\cdot)\right\}_{\varepsilon \in[0,1]}$ be a family of semigroups satisfying

(i) $T_{\varepsilon}(\cdot)$ has a global attractor $\mathscr{A}_{\varepsilon}$, for each $\varepsilon \in[0,1]$, such that $\overline{\cup_{\varepsilon \in[0,1]} \mathscr{A}_{\varepsilon}}$ is compact and $T_{\varepsilon}(\cdot)$ is reversible for all $\varepsilon \in[0,1]$.

(ii) $\mathscr{E}_{\varepsilon}=\left\{x_{1, \varepsilon}^{*}, \ldots, x_{n, \varepsilon}^{*}\right\}$ is made only hyperbolic equilibrium point of $T_{\varepsilon}(\cdot)$ for each $\varepsilon \in[0,1]$ and

$$
\operatorname{dist}_{H}\left(x_{i, \varepsilon}, x_{0, \varepsilon}\right) \stackrel{\varepsilon \rightarrow 0}{\longrightarrow} 0 .
$$

(iii) There are $\delta>0$ and $\varepsilon_{0} \in(0,1]$ such that, if $0 \leq \varepsilon \leq \varepsilon_{0}$ and $x_{\varepsilon}^{*} \in \mathscr{E}_{\varepsilon}$ then $x_{\mathcal{E}}^{*}$ is the maximal invariant set in $\mathscr{O}_{\delta}\left(x_{\varepsilon}^{*}\right)=\left\{y \in X:\left\|y-x_{\varepsilon}^{*}\right\|<\delta\right\}$. 
(iv) For each compact set $K \subset[0, \infty) \times X$ we have that

$$
\sup _{(t, x) \in K}\left\|T_{\mathcal{E}}(t) x-T_{0}(t) x\right\|_{X} \stackrel{\varepsilon \rightarrow 0}{\longrightarrow} 0 .
$$

(v) For each compact set $J \subset[0, \infty)$

$$
\sup _{t \in J} \sup _{z \in \mathscr{A}}\left\|D_{x} T_{\varepsilon}(t)(z)-D_{x} T_{0}(t)(z)\right\|_{\mathscr{L}(X)} \stackrel{\varepsilon \rightarrow 0}{\longrightarrow} 0 .
$$

(vi) $T_{0}(\cdot)$ is a Gradient and Morse-Smale semigroup.

Then there is an $\varepsilon_{1} \in\left(0, \varepsilon_{0}\right)$ such that $T_{\mathcal{E}}(\cdot)$ is a Gradient Morse-Smale semigroup for all $\varepsilon \in\left[0, \varepsilon_{1}\right]$ and there is a phase diagram isomorphism between the attractors of $T_{\varepsilon}(\cdot), \varepsilon \in\left[0, \varepsilon_{1}\right]$.

Proof. It follows from (ARAGAO-COSTA; CARABALLO; CARVALHO, 2011) that there is a $\varepsilon_{1} \in(0,1)$ such that $T_{\mathcal{E}}(\cdot)$ is gradient for $\varepsilon \in\left(0, \varepsilon_{1}\right)$. Now define $B: \mathscr{E}_{0} \rightarrow \mathscr{E}_{\mathcal{E}}$ by $B\left(x_{i, 0}^{*}\right)=x_{i, \varepsilon}^{*}$, $i=1, \ldots, n$. Then $B$ is a bijection and we can assume, by openness of the transversality property, that $\varepsilon_{1}$ is small enough so that, if $W^{u}\left(x_{i, 0}^{*}\right) \cap W_{\mathrm{loc}}^{s}\left(x_{j, 0}^{*}\right) \neq \emptyset$ then $W^{u}\left(x_{i, \varepsilon}^{*}\right) \cap W_{\mathrm{loc}}^{s}\left(x_{j, \varepsilon}^{*}\right) \neq \emptyset$ and moreover there is a point in which the intersection is transversal. This shows that if there is a connection between $x_{i, 0}^{*}$ and $x_{j, 0}^{*}$, then there is a connection between $x_{i, \varepsilon}^{*}$ and $x_{j, \varepsilon}^{*}$. On the other hand, assume that we have a sequence $\varepsilon_{k} \rightarrow 0$ and global solutions $\xi_{\varepsilon_{k}}: \mathbb{R} \rightarrow X$ of $T_{\varepsilon_{k}}(\cdot)$ such that

$$
\xi_{\varepsilon_{k}}(t) \stackrel{t \rightarrow-\infty}{\longrightarrow} x_{i, \varepsilon_{k}}^{*} \quad \text { and } \quad \xi_{\varepsilon_{k}}(t) \stackrel{t \rightarrow \infty}{\longrightarrow} x_{j, \varepsilon_{k}}^{*}
$$

Then we can construct a finite collection of global solutions $\xi_{l}: \mathbb{R} \rightarrow X$ of $T_{0}(\cdot), l=1, \ldots, m \leq n$, together with equilibrium points $e_{l}^{*} \in \mathscr{E}_{0}$, such that $e_{1}^{*}=x_{i, 0}^{*}, e_{m}^{*}=x_{j, 0}^{*}$ and

$$
\xi_{l}(t) \stackrel{t \rightarrow-\infty}{\longrightarrow} e_{l}^{*} \quad \text { and } \quad \xi_{l}(t) \stackrel{t \rightarrow \infty}{\longrightarrow} e_{l+1}^{*}, l=1, \ldots, m-1 .
$$

Now the $\lambda$-Lemma (see (BORTOLAN et al., 2015)) ensures the existence of a global solution $\xi: \mathbb{R} \rightarrow X$ of $T_{0}(\cdot)$ such that (see Figure 7)

$$
\xi(t) \stackrel{t \rightarrow-\infty}{\longrightarrow} x_{i, 0}^{*} \quad \text { and } \quad \xi(t) \stackrel{t \rightarrow \infty}{\longrightarrow} x_{j, 0}^{*}
$$

Example 2.4.6. Consider the family of scalar semilinear parabolic problems

$$
\left\{\begin{array}{l}
u_{t}+\left(p_{\varepsilon}(x) u_{x}\right)_{x}=f(u), 0<x<1 \\
u^{\varepsilon}=0, x \in\{0,1\} \\
u^{\varepsilon}(0)=u_{0}^{\varepsilon}
\end{array}\right.
$$

where, $\varepsilon \in(0,1), p_{\varepsilon} \in C^{1}([0,1])$ and $f$ is such that the equation (2.13) generates a semigroup in $H_{0}^{1}(0,1)$ with a global attractor $\mathscr{A}_{\varepsilon}$ for all $\varepsilon \in(0,1)$. In (ARRIETA; BEZERRA; CARVALHO, 


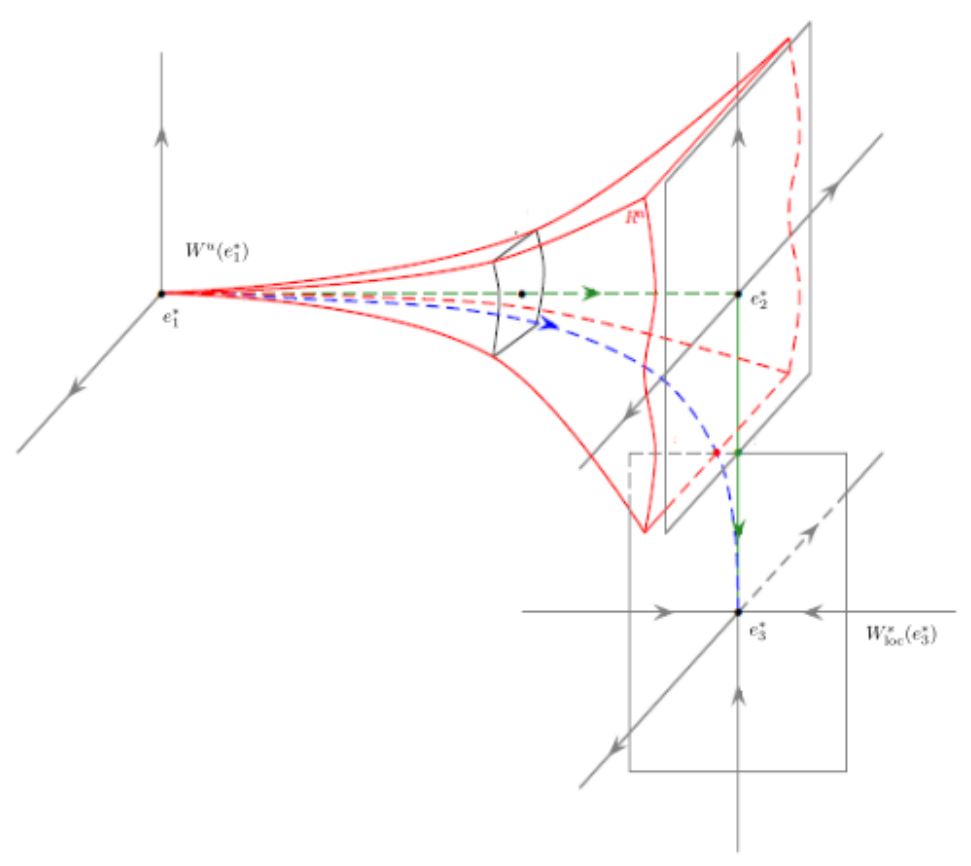

Figure 7 - Consequence of $\lambda$-lemma

2013) the author proved the properties (i)-(v) of the Theorem 2.4.5. With the same argument of (HENRY, 1985) we can prove that (2.13) with $\varepsilon=0$ generate a Morse-Smale semigroup in $H_{0}^{1}(0,1)$ see for example (CARBONE; RUAS-FILHO, 2006). Then for $\varepsilon$ sufficiently small the semigroup $T_{\mathcal{E}}(\cdot)$ generated by the solution of (2.13) is a Morse-Smale semigroup and there is a phase diagram isomorphism between the attractors.

\subsection{Shadowing in Dynamical Systems}

In this section we define the concept of Lipschitz Shadowing and we see an application to the convergence of attractors. All results stated here can be found in (SANTAMARIA; ARRIETA, 2014).

Definition 2.5.1. The discrete dynamical system generated by a function $T: X \rightarrow X$ is defined by

$$
\left\{\begin{array}{l}
T_{0}=I_{X}, \\
T_{n}=T^{n}, \quad n \in \mathbb{Z} .
\end{array}\right.
$$

If we just consider $n \in \mathbb{N}$ we say that $T_{n}$ is a discrete semigroup.

Definition 2.5.2. A trajectory (or global solution) of the discrete dynamical system generated by $T$ is a sequence $\left\{x_{n}\right\}_{n \in \mathbb{Z}} \subset X$, such that, $x_{n+1}=T\left(x_{n}\right)$ for all $n \in \mathbb{Z}$. 
Definition 2.5.3. An attractor for the map $T: X \rightarrow X$ is a set $\mathscr{A} \subset X$ which is compact, invariant $(T(\mathscr{A})=\mathscr{A})$ and attracts bounded sets of $X$, that is, for each bounded set $B \subset X$,

$$
\operatorname{dist}_{H}\left(T_{n} B, \mathscr{A}\right) \stackrel{n \rightarrow \infty}{\longrightarrow} 0 .
$$

The notions of hyperbolic fixed points, stable and unstable manifolds for a map $T$ are similar to the continuous case.

Matching with the Definition 2.4.3, a discrete semigroup generated by a function $T$ having an attractor $\mathscr{A}$ is Morse-smale if $T, D T$ are injective on $\mathscr{A}$, the non-wandering set is finite and consist of all hyperbolic points of $T$ and all fixed points of $T$ are hyperbolic with finite dimensional unstable manifolds.

Example 2.5.4. If $T(\cdot)$ is a gradient semigroup with attractor $\mathscr{A}$, if we define $T=T(1)$, then $T$ generates a discrete semigroup with the same attractor $\mathscr{A}$. In fact, if $\mathscr{A}^{\prime}$ is the attractor of $T$, since $\mathscr{A}^{\prime}$ attracts $\mathscr{A}$ by $T$ and $T_{n} \mathscr{A}=T(n) \mathscr{A}=\mathscr{A}$, we have $\mathscr{A} \subset \mathscr{A}^{\prime}$. On the other hand, if $z \in \mathscr{A}^{\prime}$, there are $x^{*} \in \mathscr{E}$ and a global solution $\xi: \mathbb{Z} \rightarrow \mathscr{A}^{\prime}$ such that $\xi(0)=z$ and $\xi(-n) \stackrel{n \rightarrow \infty}{\longrightarrow} x^{*}$. Now define $\varphi(-t)$ for all $t \geq 0$ by: for any $n \in \mathbb{N}$,

$$
\varphi(-t)=T(n-t) \xi(-n), \text { for all } 0 \leq t \leq n
$$

Thus $\varphi(\cdot)$ is a bounded solution of $T(\cdot)$ such that $\varphi(0)=z$, which implies $z \in \mathscr{A}$.

Definition 2.5.5. We say that a sequence $\left\{x_{n}\right\}_{n \in \mathbb{Z}}$ is a $\delta$-pseudo-trajectory of $T$ if

$$
\left\|T x_{x}-x_{k+1}\right\|_{X} \leq \delta, \quad \text { for all } n \in \mathbb{Z}
$$

Definition 2.5.6. We say that a point $x \in X \varepsilon$-shadows a $\delta$-pseudo-trajectory $\left\{x_{k}\right\}$ on $U \subset X$ if the inequality

$$
\left\|T^{k} x-x_{k}\right\|_{X} \leq \varepsilon, \quad k \in \mathbb{Z}
$$

holds.

Definition 2.5.7. The map $T$ has the Lipschitz Shadowing Property (LpSP) on $U \subset X$, if there are constants $L, \delta_{0}>0$ such that for any $0<\delta \leq \delta_{0}$, any $\delta$-pseudo-trajectory of $T$ in $U$ is $(L \delta)$-shadowed by a trajectory of $T$ in $X$, that is, for any sequence $\left\{x_{k}\right\}_{k} \subset U \subset X$ with

$$
\left\|T x_{k}-x_{k+1}\right\|_{X} \leq \delta \leq \delta_{0}, \quad k \in \mathbb{Z}
$$

there is a point $x \in X$ such that the inequality

$$
\left\|T^{k} x-x_{k}\right\|_{X} \leq L \delta, \quad k \in \mathbb{Z}
$$

holds. If the property holds with $U=X$, we say that $T$ has the LpSP. 
Example 2.5.8. Let $T: \mathbb{R}^{m} \rightarrow \mathbb{R}^{m}$ be a Morse Smale map which has an attractor $\mathscr{A}$. Then $T$ has the LpSP on a neighborhood $\mathscr{N}(\mathscr{A})$ of $\mathscr{A}$. In fact it is proved in (PILYUGUN, 1999) that a structurally stable dynamical system on a compact manifold has a LpSP, and we saw in the Section 2.4 that a Morse-Smale system is structurally stable. Putting this results together the authors in (SANTAMARIA; ARRIETA, 2014) proved that a Morse-Smale semigroup has the LpSP in a neighborhood of its attractor.

Since $\mathscr{A}$ is compact and has all dynamic of the system, we can restrict our attention on a neigborhood $\mathscr{N}(\mathscr{A})$ of $\mathscr{A}$, thus we consider the space $C^{1}\left(\mathscr{N}(\mathscr{A}), \mathbb{R}^{m}\right)$ with the $C^{1}$-topology.

Proposition 2.5.9. Let $T_{1}, T_{2}: X \rightarrow X$ be maps which have a global attractors $\mathscr{A}_{1}, \mathscr{A}_{2}$. Assume that $\mathscr{A}_{1}, \mathscr{A}_{2} \subset \mathscr{U} \subset X$, that $T_{1}, T_{2}$ have both the LpSP on $\mathscr{U}$, with parameters $L, \delta_{0}$ and $\| T_{1}-$ $T_{2} \|_{\mathscr{L}^{\infty}(\mathscr{U}, X)} \leq \delta$. Then we have

$$
\operatorname{dist}_{H}\left(\mathscr{A}_{1}, \mathscr{A}_{2}\right) \leq\left\|T_{1}-T_{2}\right\|_{\mathscr{L}^{\infty}(\mathscr{U}, X)} .
$$

Proof. Since $T_{1}$ and $T_{2}$ has the LpSP on $\mathscr{U}$. Take a trajectory $\left\{y_{n}\right\}_{n}$ of $T_{2}$ in $\mathscr{A}_{2}$, then $\left\{y_{n}\right\}_{n}$ is a $\delta$-psudo-trajectory of $T_{1}$ with $\delta=\left\|T_{1}-T_{2}\right\|_{\mathscr{L}^{\infty}(\mathscr{U}, X)} \leq \delta_{0}$. By LpSP there is a trajectory $\left\{x_{n}\right\}_{n} \subset X$ of $T_{1}$ such that $\left\|x_{n}-y_{n}\right\|_{X} \leq L \delta$ for al $n \in \mathbb{Z}$, hence $\left\{x_{n}\right\}_{n} \subset \mathscr{A}_{1}$. Since $\left\{y_{n}\right\}_{n}$ is arbitrary the result follows.

The arguments to obtain an estimate for convergence of attractor accomplished by (SANTAMARIA; ARRIETA, 2014) was inspired by the work (PILYUGUN, 1999) where a closed manifold was considered, hence it is necessary consider finite dimension. Some results about Shadowing in Banach space can be found in (HENRY, 1994a).

We are now ready to state the main result of this section.

Theorem 2.5.10. [Arrieta and Santamaría] Let $T: \mathbb{R}^{m} \rightarrow \mathbb{R}^{m}$ be a Morse-Smale function with a global attractor $\mathscr{A}$. Then there are a positive constant $L$, a neighborhood $\mathscr{N}(\mathscr{A})$ of $\mathscr{A}$ and a neighborhood $\mathscr{N}(T)$ of $T$ in the $C^{1}\left(\mathscr{N}(\mathscr{A}), \mathbb{R}^{m}\right)$ topology such that, for any $T_{1}, T_{2} \in \mathscr{N}(T)$ with attractors $\mathscr{A}_{1}, \mathscr{A}_{2}$ respectively, we have

$$
\operatorname{dist}_{H}\left(\mathscr{A}_{1}, \mathscr{A}_{2}\right) \leq L\left\|T_{1}-T_{2}\right\|_{L^{\infty}\left(\mathscr{N}(\mathscr{A}), \mathbb{R}^{m}\right)}
$$

Proof. By Proposition 2.5.9 we just need to prove that Morse-Smale system has the LpSP in a neighborhood of its attractor but, this is the main result stated in Chapter 2 of (PILYUGUN, 1999).

To end this section we present applications of Theorem 2.5.10 in order to improve the rate of convergence of attractor obtained in the Section 2.3. The next example can be found in (SANTAMARIA; ARRIETA, 2014). 
Example 2.5.11. Consider the set

$$
Q=\left\{(x, y) \in \mathbb{R} \times \mathbb{R}^{N-1}: 0 \leq x \leq 1,|y|<1\right\}
$$

The thin domain is defined by

$$
Q_{\varepsilon}=\left\{(x, \varepsilon y) \in \mathbb{R} \times \mathbb{R}^{N-1}:(x, y) \in Q\right\}, \quad \varepsilon \in(0,1] .
$$

This domain is obtained by shrinking the set $Q$ by a factor $\varepsilon$ in the $N-1$ direction given by the variable $y$. When $\varepsilon$ goes to zero, $Q_{\varepsilon}$ tends for the one dimensional segment $(0,1)$.

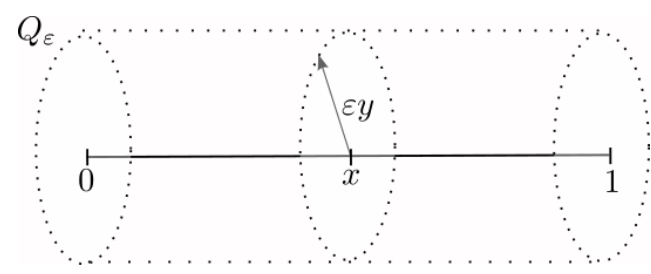

Figure 8 - Thin Domain

We consider the reaction-diffusion equation

$$
\left\{\begin{array}{l}
u_{t}-\Delta u+\alpha u=f(u), \text { in } Q_{\varepsilon} \\
\frac{\partial u}{\partial v_{\varepsilon}}=0, \text { in } \partial Q_{\varepsilon}
\end{array}\right.
$$

where $\alpha>0$ is a fixed number, $v_{\varepsilon}$ the unit outward normal to $\partial Q_{\varepsilon}$ and $f: \mathbb{R} \rightarrow \mathbb{R}$ a $C^{2}$-function satisfying

(i) $\left|f^{\prime}(s)\right| \leq C\left(1+|s|^{\rho-1}\right), s \in \mathbb{R}$, for some $\rho>1$,

(ii) $f(s) s \leq 0,|s| \geq M$, for some $M>0$.

The limiting problem of (2.14) as $\varepsilon \rightarrow 0$ is given by

$$
\left\{\begin{array}{l}
u_{t}-u_{x x}+\alpha u=f(u), \text { in }(0,1), \\
u_{x}(0)=u_{x}(1)=0 .
\end{array}\right.
$$

The equation (2.14) is well posed in $H^{1}\left(Q_{\varepsilon}\right)$ and the equation (2.15) is well posed in $H^{1}(0,1)$, that is, if we define the operators $A_{\varepsilon}=-\Delta u+\alpha u$ and $A_{0} u=-u_{x x}+\alpha u$, the solutions of $u_{t}+A_{\varepsilon} u^{\varepsilon}=f\left(u^{\varepsilon}\right)$ generate a semigroup $T_{\varepsilon}(\cdot)$, for all $\varepsilon \in[0,1]$ and this semigroups have a global attractor $\mathscr{A}_{\varepsilon}$, for all $\varepsilon \in[0,1]$. Moreover $T_{0}(\cdot)$ is a Morse-Smale semigroup.

In order to understand the attractor $\mathscr{A}_{0}$ in $H^{1}\left(Q_{\varepsilon}\right)$, we define $E_{\varepsilon}: H^{1}(0,1) \rightarrow H^{1}\left(Q_{\varepsilon}\right)$ by $\left(E_{\varepsilon} u\right)(x, y)=u(x)$, for all $u \in H^{1}(0,1)$.

Assuming that the equilibrium points of (2.15) are hyperbolic, the authors in (SANTAMARIA; ARRIETA, 2014) proved the following results 
(i) $\left\|A_{\varepsilon}^{-1}-E_{\varepsilon} A_{0}^{-1}\right\|_{\mathscr{L}\left(L^{2}, H^{1}\right)} \leq C \varepsilon$;

(ii) $\left\|T_{\varepsilon}(1)-E_{\varepsilon} T_{0}(1)\right\|_{\mathscr{L}\left(H^{1}\right)} \leq C \varepsilon|\log (\varepsilon)|$;

(iii) There is a finite dimensional invariant manifold $\mathscr{M}_{\varepsilon}$ given by graph of Lipschitz functions $s_{\varepsilon}$ such that $\mathscr{A}_{\mathcal{E}} \subset \mathscr{M}_{\varepsilon}$ and the flow can be reduced to finite dimension, that is, we can consider an ODE generating a semigroup $\bar{T}_{\varepsilon}(\cdot)$ in $\mathbb{R}^{m}$ with an attractor $\overline{\mathscr{A}}_{\varepsilon}$, where $m=\operatorname{dim}\left(\mathscr{M}_{\varepsilon}\right)$. Moreover we have the following estimates

(a) $\left\|\bar{T}_{\mathcal{E}}(1)-\bar{T}_{0}(1)\right\|_{\mathscr{L}\left(\mathbb{R}^{m}\right)} \leq C \varepsilon|\log (\varepsilon)|$;

(b) $\sup _{v \in \mathbb{R}^{m}}\left\|s_{\mathcal{\varepsilon}}(v)-s_{0}(v)\right\|_{H^{1}} \leq C \varepsilon|\log (\varepsilon)|$.

Therefore the convergence of attractors of (2.14) and (2.15) can be estimated by

$$
\mathrm{d}_{H}\left(\mathscr{A}_{\varepsilon}, E_{\varepsilon} \mathscr{A}_{0}\right) \leq C \varepsilon^{\frac{N+1}{2}}|\log (\varepsilon)|
$$

where $C$ is a constant independent of $\varepsilon$.

Example 2.5.12. Consider the problem in the Example 2.3.6 in a scalar version.

$$
\left\{\begin{array}{l}
u_{t}^{\varepsilon}-\left(p_{\varepsilon}(x) u_{x}^{\varepsilon}\right)_{x}=f\left(u^{\varepsilon}\right), x \in(0,1), t>0 \\
u^{\varepsilon}(t, x)=0, x \in\{0,1\}, t>0 \\
u^{\varepsilon}(0, x)=u_{0}^{\varepsilon}(x)
\end{array}\right.
$$

Since the problem is well posed in $H_{0}^{1}(0,1)$ and the limiting problem is Morse-Smale, we can follow the steps of the Example 2.5.11 to obtain

$$
d_{H}\left(\mathscr{A}_{\varepsilon}, \mathscr{A}_{0}\right) \leq C\left\|p_{\varepsilon}-p_{0}\right\|_{L^{\infty}}\left|\log \left(\left\|p_{\varepsilon}-p_{0}\right\|_{L^{\infty}}\right)\right|,
$$

for some constant $C$ independent of $\varepsilon$. Note that (2.16) improves (2.11). 


\section{SEMILINEAR PROBLEMS WHOSE DYNAMICS IS DICTATED BY AN ODE}

There are many parabolic problems whose asymptotic behavior is dictated by a system of Morse-Smale ordinary differential equations, for example, reaction diffusion equation where the diffusion coefficient become very large in all domain or reaction diffusion equation where the diffusion coefficient is very large except in a neighborhood of a finite number of points where it becomes small. These kind of problems was considered in the works (CARVALHO et al., 2010; CARVALHO; PEREIRA, 1994; CARVALHO; PRIMO, 2004; CONLEY; HOFF; SMOLLER, 1978; FUSCO, 1987) and (HALE, 1986), where well-posedness, functional setting and convergence of attractors were studied. In general it is considered a family of parabolic problem depending on a positive parameter $\varepsilon$ and when $\varepsilon$ converges to a value $\bar{\varepsilon}$ that could be 0 or $\infty$, it is obtained a limiting ordinary differential equation that contains all dynamics of the problem. Therefore the partial differential equation that generated an abstract parabolic problem in an infinite dimensional phase space can be considered in large time behavior as an ordinary differential equation in a finite dimensional space.

One of the most important part in these works is the study of an eigenvalue problem to determine that the first eigenvalues converge to matching eigenvalues of the limiting ODE, whereas all the others blow up, establishing the existence of a large gap between the eigenvalues as the parameter $\varepsilon$ goes to $\bar{\varepsilon}$. The large gap between the eigenvalues is known as gap condition. It is the main property that enable us construct an invariant manifold given as graph of certain Lipschitz map defined in a finite dimensional space that becomes flat, as the parameter varies. These manifolds are differentiable and since they are invariant we can restrict the flow to them and project the flow in a finite dimensional space. The theory of invariant manifold is well developed in many works, see (BERNAL, 1993; CARVALHO; PRIMO, 2004; HENRY, 1980) and (SELL; YOU, 2002).

The main property about Morse-Smale problems is structural stability under small $C^{1}$ 
perturbation, thus it is interesting treat the problems described above as singular perturbation of a Morse-Smale limiting ODE, therefore there is an isomorphism between the attractors in the sense that all equilibrium points and connections between them are preserved, see (BORTOLAN et al., 2015).

We are interested in knowing how fast the dynamics above approach each other, more precisely, we want to estimate the convergence of attractors when the parameter $\varepsilon$ converges to $\bar{\varepsilon}$. In this direction we follow the works (ARRIETA; BEZERRA; CARVALHO, 2013; BABIN; VISHIK, 1992) and (SANTAMARIA; ARRIETA, 2014), where a rate of convergence for the attractors was obtained assuming a rate of convergence for the resolvent operators. However the rates obtained in these works always show a loss with respect to rate of resolvent operators considered. In this Chapter we will show that for a class of parabolic problems that can be regarded as an ordinary differential equation we do not have loss in the process to pass the convergence of the resolvents operators to convergence of attractors.

This chapter is organized as follows. In the Section 3.1 we developed an abstract functional framework to treat the parabolic problem whose asymptotic behavior is described by a system of ordinary differential equation, we introduce some notation and define some notions of theory of attractor for semigroups. In the Section 3.2 we recall the notion of compact convergence and prove the convergence of eigenvalues and eigenfunctions, we also obtain the gap condition and estimates a priori on linear semigroups that is essential to construct the invariant manifold. In the Sections 3.3 and 3.4 we show that the rate of convergence of resolvents operators is the same rate of convergence of invariant manifolds and attractors. The Section 3.5 is devoted to comments about more general situations that can be considered.

\subsection{Functional Setting}

In this section we introduce the general framework that we use to treat parabolic problems whose asymptotic behavior is dictated by a system of Morse-Smale ordinary differential equations. We consider here only positive self-adjoint operator with compact resolvent. More general situation will be addressed in the Section 3.5.

Let $X_{0}$ be a finite dimensional Hilbert space with $\operatorname{dim}\left(X_{0}\right)=n$, for some positive integer $n$, and let $A_{0}: X_{0} \rightarrow X_{0}$ be an invertible bounded linear operator whose spectrum set $\sigma\left(A_{0}\right)$ is given by

$$
\sigma\left(A_{0}\right)=\left\{\lambda_{1}^{0}<\lambda_{2}^{0}<\cdots<\lambda_{n}^{0}\right\}, \quad 0<\lambda_{1}^{0}
$$

Consider the following system of ordinary differential equation

$$
\left\{\begin{array}{l}
\dot{u}^{0}+A_{0} u^{0}=f_{0}\left(u^{0}\right) \\
u^{0}(0)=u_{0}^{0} \in X_{0},
\end{array}\right.
$$

where $f_{0}: X_{0} \rightarrow X_{0}$ is continuously differentiable. 
Let $\left\{X_{\varepsilon}\right\}_{\varepsilon \in\left(0, \varepsilon_{0}\right]}, \varepsilon_{0}>0$, be a family of separable Hilbert spaces and let $\left\{A_{\varepsilon}\right\}_{\varepsilon \in\left(0, \varepsilon_{0}\right]}$ be a family of invertible linear operators such that, for each $\varepsilon \in\left(0, \varepsilon_{0}\right], A_{\varepsilon}: D\left(A_{\varepsilon}\right) \subset X_{\varepsilon} \rightarrow X_{\varepsilon}$ is self-adjoint, positive and has compact resolvent, hence its spectrum $\sigma\left(A_{\varepsilon}\right)$ is discrete, real and consists only eigenvalues with finite multiplicity. We denote

$$
\sigma\left(A_{\varepsilon}\right)=\left\{\lambda_{1}^{\varepsilon}<\lambda_{2}^{\varepsilon}<\cdots\right\}, \quad 0<\lambda_{1}^{\varepsilon},
$$

and we consider $\left\{\varphi_{j}^{\varepsilon}\right\}_{j=1}^{\infty}$ the associated orthonormal family of eigenfunctions such that $Q_{j}^{\varepsilon}$ is the orthogonal projection onto $\operatorname{span}\left[\varphi_{1}^{\varepsilon}, \cdots, \varphi_{j}^{\varepsilon}\right]$. According to Section 2.2 in chapter 2 we can define the fractional powers of $A_{\varepsilon}$, that is, for $\alpha \in(0,1), A^{\alpha}$ is defined by

$$
A_{\varepsilon}^{\alpha} u^{\varepsilon}=\sum_{j=1}^{\infty}\left(\lambda_{j}^{\varepsilon}\right)^{\alpha} Q_{j}^{\varepsilon} u^{\varepsilon}, \quad \forall u^{\varepsilon} \in D\left(A_{\varepsilon}^{\alpha}\right),
$$

where

$$
D\left(A_{\varepsilon}^{\alpha}\right)=\left\{u \in X_{\varepsilon}: \sum_{j=1}^{\infty}\left(\lambda_{j}^{\varepsilon}\right)^{2 \alpha}\left\|Q_{j}^{\varepsilon} u\right\|_{X_{\varepsilon}}^{2}<\infty\right\} .
$$

It is well known that $A_{\varepsilon}^{\alpha}$ is a positive self-adjoint operator and $D\left(A_{\varepsilon}^{\alpha}\right)$ becomes a Hilbert space, denoted by $X_{\varepsilon}^{\alpha}$, when equipped with the inner product $\langle u, v\rangle_{X_{\varepsilon}^{\alpha}}=\left\langle A_{\varepsilon}^{\alpha} u, A_{\varepsilon}^{\alpha} v\right\rangle_{X_{\varepsilon}}, u, v \in X_{\varepsilon}^{\alpha}$. The corresponding norm is

$$
\|u\|_{X_{\varepsilon}^{\alpha}}=\left\|A_{\varepsilon}^{\alpha} u\right\|_{X_{\varepsilon}}=\left(\sum_{j=1}^{\infty}\left(\lambda_{j}^{\varepsilon}\right)^{2 \alpha}\left\|Q_{j}^{\varepsilon} u\right\|_{X_{\varepsilon}}^{2}\right)^{\frac{1}{2}} .
$$

Consider the following abstract parabolic problem,

$$
\left\{\begin{array}{l}
u_{t}^{\varepsilon}+A_{\varepsilon} u^{\varepsilon}=f_{\varepsilon}\left(u^{\varepsilon}\right) \\
u^{\varepsilon}(0)=u_{0}^{\varepsilon} \in X_{\varepsilon}^{\alpha}
\end{array}\right.
$$

where $f_{\varepsilon}: X_{\varepsilon}^{\alpha} \rightarrow X_{\varepsilon}$ is continuously differentiable such that

$$
\sup _{\varepsilon \in\left(0, \varepsilon_{0}\right]} \sup _{u \in X_{\varepsilon}^{\alpha}}\left\|f_{\varepsilon}(u)\right\|_{X_{\varepsilon}} \leq C
$$

and

$$
\left\|f_{\varepsilon}(u)-f_{\varepsilon}(v)\right\|_{X_{\varepsilon}} \leq C\|u-v\|_{X_{\varepsilon}^{\alpha}}, \forall u, v \in X_{\varepsilon}^{\alpha},
$$

where $C$ is a constant independent of $\varepsilon$.

Well-posedness for abstract parabolic problem type (3.2) is developed in (CARVALHO; LANGA; ROBINSON, 2010) and for ordinary differential equations type (3.1) in (HALE, 2009). Basically, with some growth, signal and dissipativeness conditions, we can assume that the solution $u^{0}(\cdot)$ of (3.1) and $u^{\varepsilon}(\cdot)$ of (3.2) are uniquely determined and defined for all $t \geq 0$. The super index $\varepsilon$ makes reference to the elliptic operator $A_{\varepsilon}$ and in order to treat the problems (3.1) and (3.2) in a coupled form we will consider $\varepsilon \in\left[0, \varepsilon_{0}\right]$ with the convention $X_{0}^{\alpha}=X_{0}$. 
For each $\varepsilon \in\left[0, \varepsilon_{0}\right]$ we define $T_{\varepsilon}(t) u_{0}^{\varepsilon}=u^{\varepsilon}(t), t>0$ and $T_{\varepsilon}(0)=I_{X_{\varepsilon}^{\alpha}}$, where $I_{X_{\varepsilon}^{\alpha}}$ denotes the identity in $X_{\varepsilon}^{\alpha}$. We have $T_{\mathcal{\varepsilon}}(\cdot): X_{\mathcal{\varepsilon}}^{\alpha} \rightarrow X_{\mathcal{\varepsilon}}^{\alpha}$ a family of nonlinear semigroups satisfying the variation constants formula,

$$
T_{\varepsilon}(t) u_{0}^{\varepsilon}=e^{-A_{\varepsilon} t} u_{0}^{\varepsilon}+\int_{0}^{t} e^{-A_{\varepsilon}(t-s)} f_{\mathcal{\varepsilon}}\left(T_{\mathcal{\varepsilon}}(s) u_{0}^{\varepsilon}\right) d s, t>0, u_{0}^{\varepsilon} \in X_{\mathcal{\varepsilon}}^{\alpha}, \varepsilon \in\left[0, \varepsilon_{0}\right],
$$

where $\left\{e^{-A_{\varepsilon} t}: t \geq 0\right\}$ is the linear semigroup generated by $-A_{\varepsilon}$.

Since $A_{0}$ is a bounded operator in finite dimensional space, $e^{-A_{0} t}$ is an uniformly continuous semigroup and for $\varepsilon \in\left(0, \varepsilon_{0}\right], e^{-A_{\varepsilon} t}$ is a strongly continuous semigroup given by

$$
e^{-A_{\varepsilon} t} u_{0}^{\varepsilon}=\sum_{j=1}^{\infty} e^{-\lambda_{j}^{\varepsilon} t} Q_{j}^{\varepsilon} u_{0}^{\varepsilon}=\frac{1}{2 \pi i} \int_{\Gamma} e^{\lambda t}\left(\lambda+A_{\varepsilon}\right)^{-1} u_{0}^{\varepsilon} d \lambda, t>0,
$$

where $\Gamma$ is the boundary of the set $\{\lambda \in \mathbb{C}:|\arg (\lambda)| \leq \phi\} \backslash\{\lambda \in \mathbb{C}|\lambda| \leq r\}$, for some $\phi \in$ $(\pi / 2, \pi)$ and $r>0$, oriented in such a way that the imaginary part is increasing.

We assume that the nonlinear semigroups $T_{\varepsilon}(\cdot), \varepsilon \in\left[0, \varepsilon_{0}\right]$, satisfying (3.3) have a global attractor $\mathscr{A}_{\varepsilon}$ uniformly bounded, that is,

$$
\sup _{u \in \mathscr{A} \varepsilon}\|u\|_{X_{\varepsilon}^{\alpha}} \leq C
$$

for some constant $C$ independent of $\varepsilon$.

Finally we also assume that the system (3.1) generated a Morse-Smale semigroup in $X_{0}$. Thus, if we denote $\mathscr{E}_{0}$ the set of equilibrium points of $T_{0}(\cdot)$, then it is composed of $p$ hyperbolic points, that is,

$$
\mathscr{E}_{0}=\left\{u \in X_{0}: A_{0} u-f_{0}(u)=0\right\}=\left\{u_{1}^{0, *}, \ldots, u_{p}^{0, *}\right\},
$$

and $\sigma\left(A_{0}-f_{0}^{\prime}\left(u_{i}^{0, *}\right)\right) \cap\left\{u \in X_{0}:\|u\|_{X_{0}}=1\right\}=\emptyset$, for $i=1, \ldots, p$. Moreover $T_{0}(\cdot)$ is dynamically gradient and

$$
\mathscr{A}_{0}=\bigcup_{i=1}^{p} W^{u}\left(u_{i}^{0, *}\right),
$$

where $W^{u}\left(u_{i}^{0, *}\right)$ is the unstable manifold associated with the equilibrium point $u_{i}^{0, *} \in \mathscr{E}_{0}$ and for $i \neq j$ the unstable manifold $W^{u}\left(u_{i}^{0, *}\right)$ and the local stable manifold $W_{\text {loc }}^{s}\left(u_{j}^{0, *}\right)$ has tranversal intersection, see Section 2.4 in Chapter 2.

\subsection{Compact Convergence}

In this section we recall the notion of compact convergence and we assert the necessary assumptions for estimate the convergence of attractors of (3.1) and (3.2). In fact, we assume that the resolvents operators and the nonlinearities have a rate of convergence and we used it to estimate the convergence of the first eigenvalues and associated eigenfunctions, consequently we also estimate the convergence of spectral projections. All necessary conditions to construct the invariant manifold in the next section will be made here. 
Assume that there are two families of bounded linear operators $E_{\varepsilon}: X_{0} \rightarrow X_{\varepsilon}^{\alpha}$ and $M_{\varepsilon}: X_{\varepsilon} \rightarrow X_{0}, \varepsilon \in(0, \varepsilon]$, satisfying the following properties,

(i) $M_{\varepsilon} \circ E_{\varepsilon}=I_{X_{0}}$;

(ii) $\left\|E_{\varepsilon} u^{0}\right\|_{X_{\varepsilon}} \rightarrow\left\|u^{0}\right\|_{X_{0}}$ as $\varepsilon \rightarrow 0$;

(iii) $\left\|E_{\varepsilon}\right\|_{\mathscr{L}\left(X_{0}, X_{\varepsilon}\right)},\left\|M_{\mathcal{\varepsilon}}\right\|_{\mathscr{L}\left(X_{\varepsilon}, X_{0}\right)},\left\|E_{\varepsilon}\right\|_{\mathscr{L}\left(X_{0}, X_{\varepsilon}^{\alpha}\right)},\left\|M_{\mathcal{\varepsilon}}\right\|_{\mathscr{L}\left(X_{\varepsilon}^{\alpha}, X_{0}\right)} \leq C$, for some constant $C$ independent of $\varepsilon$.

Note that $E_{\varepsilon}$ is injective and $M_{\varepsilon}$ is surjective. Moreover the following inequalities are valid

$$
C^{-1}\left\|u^{0}\right\|_{X_{0}} \leq\left\|E_{\varepsilon} u^{0}\right\|_{X_{\varepsilon}^{\alpha}} \leq C\left\|u^{0}\right\|_{X_{0}}, \quad u^{0} \in X_{0} .
$$

Definition 3.2.1. We say that a family $\left\{u^{\varepsilon}\right\}_{\varepsilon \in\left(0, \varepsilon_{0}\right]}$, whith $u^{\varepsilon} \in X_{\mathcal{\varepsilon}}^{\alpha}$, E-converge to $u^{0} \in X_{0}$ as $\varepsilon \rightarrow 0$ if $\left\|u^{\varepsilon}-E_{\varepsilon} u^{0}\right\|_{X_{\varepsilon}^{\alpha}} \rightarrow 0$ as $\varepsilon \rightarrow 0$. In that case we denote $u^{\varepsilon} \stackrel{\mathrm{E}}{\longrightarrow} u^{0}$.

With this notion of convergence we recall the notion of compactness.

Definition 3.2.2. We say that a sequence $\left\{u^{\varepsilon_{k}}\right\}_{k \in \mathbb{N}}$, with $u^{\varepsilon_{k}} \in X_{\varepsilon_{k}}$, is relatively compact if for each subsequence $\left\{u^{\varepsilon_{k_{l}}}\right\}_{l \in \mathbb{N}}$ there is a subsequence $\left\{u^{\varepsilon_{k_{j}}}\right\}_{j \in \mathbb{N}}$ and an element $u^{0} \in X_{0}$ such that $u^{\varepsilon_{k_{j}}} \stackrel{\mathrm{E}}{\longrightarrow} u^{0}$. The family $\left\{u^{\varepsilon}\right\}_{\varepsilon \in\left(0, \varepsilon_{0}\right]}$, with $u^{\varepsilon} \in X_{\varepsilon}^{\alpha}$ is relatively compact if any subsequence $\left\{u^{\varepsilon_{k}}\right\}_{k \in \mathbb{N}}$ is relatively compact.

In what follows $\left\{A_{\varepsilon}\right\}_{\varepsilon \in\left[0, \varepsilon_{0}\right]}$ is the family of operators defined in the previous section.

Definition 3.2.3. We say that the family of bounded linear operators $\left\{A_{\varepsilon}^{-1}\right\}_{\varepsilon \in\left(0, \varepsilon_{0}\right]}$ converges compactly to $A_{0}^{-1}$ as $\varepsilon \rightarrow 0$, which we denote $A_{\varepsilon}^{-1} \stackrel{\mathrm{CC}}{\longrightarrow} A_{0}^{-1}$, if the following two conditions are satisfied:

(i) $u^{\varepsilon} \stackrel{\mathrm{E}}{\longrightarrow} u^{0} \Rightarrow A_{\varepsilon}^{-1} u^{\varepsilon} \stackrel{\mathrm{E}}{\longrightarrow} A_{0}^{-1} u^{0}$.

(ii) each family of the form $\left\{A_{\varepsilon}^{-1} u^{\varepsilon}\right\}_{\varepsilon \in\left(0, \varepsilon_{0}\right]}$, with $\left\|u^{\varepsilon}\right\|_{X_{\varepsilon}^{\alpha}}=1$, for all $\varepsilon \in\left(0, \varepsilon_{0}\right]$, is relatively compact.

Lemma 3.2.4. Assume that $A_{\varepsilon}^{-1} \stackrel{\mathrm{CC}}{\longrightarrow} A_{0}^{-1}$. The following assertions are true.

(i) $\left\|A_{\varepsilon}^{-1}\right\|_{\mathscr{L}\left(X_{\varepsilon}^{\alpha}\right)} \leq C$, for some constant $C$ independent of $\varepsilon$.

(ii) Given any compact $K \subset \rho\left(-A_{0}\right)$ we have for $\varepsilon$ sufficiently small (we still denote $\varepsilon \in$ $\left.\left(0, \varepsilon_{0}\right]\right), K \subset \rho\left(-A_{\varepsilon}\right)$ and

$$
\sup _{\varepsilon \in\left(0, \varepsilon_{0}\right]} \sup _{\lambda \in K}\left\|\left(\lambda+A_{\varepsilon}\right)^{-1}\right\|_{\mathscr{L}\left(X_{\varepsilon}, X_{\varepsilon}^{\alpha}\right)} \leq C,
$$

for some constant $C$ independent of $\varepsilon$. Moreover $\left(\lambda+A_{\varepsilon}\right)^{-1} \stackrel{\mathrm{CC}}{\longrightarrow}\left(\lambda+A_{0}\right)^{-1}$, for all $\lambda \in K$. 
Proof. If there are sequences $\varepsilon_{k} \rightarrow 0$ and $u^{\varepsilon_{k}} \in X_{\varepsilon_{k}}^{\alpha}$ such that $\left\|u^{\varepsilon_{k}}\right\|_{X_{\varepsilon_{k}}^{\alpha}}=1$ and $\left\|A_{\varepsilon_{k}}^{-1} u^{\varepsilon_{k}}\right\|_{X_{\varepsilon_{k}}^{\alpha}} \rightarrow \infty$ as $\varepsilon \rightarrow 0$, then the condition (ii) in Definition 3.2.3 cannot be valid. Hence (i) is proved.

For the proof of (ii), if $K \cap \sigma\left(-A_{\varepsilon}\right) \neq \emptyset$, then we can take sequences $\lambda_{k} \in K \cap \sigma\left(-A_{\varepsilon}\right)$ such that $\lambda_{k} \rightarrow \lambda \in K$ and $u^{\varepsilon_{k}} \in X_{\varepsilon_{k}}$ such that $\left\|u^{\varepsilon_{k}}\right\|_{X_{\varepsilon_{k}}^{\alpha}}=1$ and $u^{\varepsilon_{k}}=\lambda_{k} A_{\varepsilon_{k}}^{-1} u^{\varepsilon_{k}}$. By condition (ii) in the Definition 3.2.3 we can assume $u^{\varepsilon_{k}}=\lambda_{k} A_{\varepsilon_{k}}^{-1} u^{\varepsilon_{k}} \stackrel{E}{\longrightarrow} \lambda u^{0}$, for some $u^{0} \in X_{0}$, and by condition (i) in Definition 3.2.3 we obtain $A_{\varepsilon_{k}}^{-1} u^{\varepsilon_{k}} \stackrel{E}{\longrightarrow} A_{0}^{-1} \lambda u^{0}$. It follows from uniqueness of the limit that $A_{0} u^{0}=\lambda u^{0}$, that is, $\lambda \in \sigma\left(-A_{0}\right) \cap K$ which is a contradition. Hence $K \subset \rho\left(-A_{\varepsilon}\right)$.

Now, assume that (3.4) fails. We claim that for $\varepsilon$ sufficiently small,

$$
\sup _{\varepsilon \in\left(0, \varepsilon_{0}\right]} \sup _{\lambda \in K}\left\|\left(I+\lambda A_{\varepsilon}^{-1}\right)^{-1}\right\|_{\mathscr{L}\left(X_{\varepsilon}, X_{\varepsilon}^{\alpha}\right)} \leq C .
$$

In fact, we have $\left(\lambda+A_{\varepsilon}\right)=A_{\varepsilon}\left(I+\lambda A_{\varepsilon}^{-1}\right)$ which implies the existence of $\left(I+\lambda A_{\varepsilon}^{-1}\right)^{-1}$, and then $\operatorname{Kernel}\left(I+\lambda A_{\varepsilon}^{-1}\right)=\{0\}$. Since $A_{\varepsilon}^{-1}$ is compact, it follows from Fredholm Alternative that $\operatorname{Kernel}\left(I+\lambda A_{\mathcal{\varepsilon}}^{-1}\right)=\{0\}$ if only if, $\mathrm{R}\left(I+\lambda A_{\varepsilon}^{-1}\right)=X_{\varepsilon}^{\alpha}$. Thus (3.5) is equivalent to following assertion

$$
\left\|\left(I+\lambda A_{\varepsilon}^{-1}\right) u^{\varepsilon}\right\|_{X_{\varepsilon}^{\alpha}} \geq \frac{1}{C}, \quad u^{\varepsilon} \in X_{\varepsilon}^{\alpha}, \quad\left\|u^{\varepsilon}\right\|_{X_{\varepsilon}^{\alpha}}=1 .
$$

Assume that (3.6) is false. With the same argument above we can obtain $u^{0} \in X_{0}$ such that $\left(I+\lambda A_{0}^{-1}\right) u^{0}=0$ which is a contradition. Hence (3.5) is valid and thus (3.4) follows noticing that

$$
\left\|\left(\lambda+A_{\varepsilon}\right)^{-1}\right\|_{\mathscr{L}\left(X_{\varepsilon}, X_{\varepsilon}^{\alpha}\right)} \leq\left\|\left(I+\lambda A_{\varepsilon}^{-1}\right)^{-1}\right\|_{\mathscr{L}\left(X_{\varepsilon}, X_{\varepsilon}^{\alpha}\right)}\left\|_{A_{\varepsilon}^{-1}}\right\|_{\mathscr{L}\left(X_{\varepsilon}, X_{\varepsilon}^{\alpha}\right)}, \quad \forall \lambda \in K, \quad \varepsilon \in\left(0, \varepsilon_{0}\right] .
$$

Finally, we prove that $\left(\lambda+A_{\varepsilon}\right)^{-1}$ converges compactly to $\left(\lambda+A_{0}\right)^{-1}$, for all $\lambda \in K$. We denote $w^{\varepsilon}=\left(I+\lambda A_{\varepsilon}^{-1}\right)^{-1} u^{\varepsilon}$, where $u^{\varepsilon} \in X_{\varepsilon}^{\alpha}$ and $\left\|u^{\varepsilon}\right\|_{X_{\varepsilon}^{\alpha}}=1$, thus $\left(\lambda+A_{\varepsilon}\right)^{-1} u^{\varepsilon}=A_{\varepsilon}^{-1} w^{\varepsilon}$ and $w^{\varepsilon}$ is uniformly bounded. Hence we can assume $\left(\lambda+A_{\varepsilon}\right)^{-1} u^{\varepsilon} \stackrel{E}{\longrightarrow} v^{0} \in X_{0}$. Thus, if $u^{\varepsilon} \stackrel{E}{\longrightarrow} u^{0}$ then $A_{\varepsilon}^{-1} u^{\varepsilon} \stackrel{E}{\longrightarrow} A_{0}^{-1} u^{0}$ and

$$
z^{\varepsilon}=\left(I+\lambda A_{\varepsilon}^{-1}\right)^{-1} A_{\varepsilon}^{-1} u^{\varepsilon}=\left(\lambda+A_{\varepsilon}\right)^{-1} u^{\varepsilon} \stackrel{E}{\longrightarrow} v^{0}
$$

which implies

$$
A_{\varepsilon}^{-1} u^{\varepsilon}=\left(I+\lambda A_{\varepsilon}^{-1}\right) z^{\varepsilon} \stackrel{E}{\longrightarrow}\left(I+\lambda A_{0}^{-1}\right) v^{0} .
$$

Therefore $A_{0}^{-1} u^{0}=\left(I+\lambda A_{0}^{-1}\right) v^{0}$, that is, $v^{0}=\left(\lambda+A_{0}\right)^{-1}$.

In what follows we assume $A_{\varepsilon}^{-1} \stackrel{\mathrm{CC}}{\longrightarrow} A_{0}^{-1}$. Moreover, we assume that there are two positive increasing functions $\tau, \rho:\left[0, \varepsilon_{0}\right] \rightarrow[0, \infty)$ such that $\tau(0)=0=\rho(0)$,

$$
\left\|A_{\varepsilon}^{-1}-E_{\varepsilon} A_{0}^{-1} M_{\mathcal{\varepsilon}}\right\|_{\mathscr{L}\left(X_{\varepsilon}, X_{\varepsilon}^{\alpha}\right)} \leq \tau(\varepsilon),
$$

and

$$
\left\|f_{\varepsilon}\left(u^{\varepsilon}\right)-E_{\varepsilon} f_{0}\left(u^{0}\right)\right\|_{X_{\varepsilon}} \leq C\left\|u^{\varepsilon}-E_{\varepsilon} u^{0}\right\|_{X_{\varepsilon}^{\alpha}}+\rho(\varepsilon), u^{\varepsilon} \in X_{\varepsilon}^{\alpha}, u^{0} \in X_{0},
$$

where $C$ is a constant independent of $\varepsilon$. 
Lemma 3.2.5. If $\lambda \in \rho\left(-A_{\varepsilon}\right)$ is such that $\lambda \notin\left(-\infty,-\lambda_{1}^{0}\right]$, then there is $\phi \in\left(\frac{\pi}{2}, \pi\right)$ such that $\lambda \in \Sigma_{-\lambda_{1}^{0}, \phi} \backslash B_{r}\left(-\lambda_{1}^{0}\right)=\left\{\mu \in \mathbb{C}:\left|\arg \left(\mu+\lambda_{1}^{0}\right)\right| \leq \phi\right\} \backslash\left\{\mu \in \mathbb{C}:\left|\mu+\lambda_{1}^{0}\right| \leq r\right\}$ for some small $r>0$, and it is valid the following estimate,

$$
\left\|\left(\lambda+A_{\varepsilon}\right)^{-1}-E_{\varepsilon}\left(\lambda+A_{0}\right)^{-1} M_{\varepsilon}\right\|_{\mathscr{L}\left(X_{\varepsilon}, X_{\varepsilon}^{\alpha}\right)} \leq C \tau(\varepsilon),
$$

where $C$ is a positive constant independent of $\varepsilon$ and $\lambda$. Moreover, if a compact set $K \subset \rho\left(-A_{\mathcal{\varepsilon}}\right) \cap$ $\rho\left(-A_{0}\right)$ then (3.9) is valid for some constant $C$ independent of $\varepsilon$ and $\lambda \in K$.

Proof. We have the following identity

$$
A_{\varepsilon}^{\alpha}\left(\left(\lambda+A_{\varepsilon}\right)^{-1}-E_{\varepsilon}\left(\lambda+A_{0}\right)^{-1} M_{\varepsilon}\right)=A_{\varepsilon}\left(\lambda+A_{\varepsilon}\right)^{-1} A_{\varepsilon}^{\alpha}\left(A_{\varepsilon}^{-1}-E_{\varepsilon} A_{0}^{-1} M_{\varepsilon}\right)\left(\lambda+A_{0}\right)^{-1}
$$

and the result follows from (3.7) by noting that $A_{\mathcal{\varepsilon}}\left(\lambda+A_{\mathcal{E}}\right)^{-1}=I-\lambda\left(\lambda+A_{\mathcal{E}}\right)^{-1}$ is uniformly bounded.

Now we will see how the convergence of the resolvent operators (3.9) implies the convergence of the eigenvalues, eigenfunctions and spectral projections.

Proposition 3.2.6. The following assertions are valid.

(i) Given $\delta>0$ sufficiently small, for $j=1, \cdots, n$, the operators

$$
Q_{\varepsilon}\left(\lambda_{j}^{0}\right)=\frac{1}{2 \pi i} \int_{\left|\xi+\lambda_{j}^{0}\right|=\delta}\left(\lambda+A_{\varepsilon}\right)^{-1} d \lambda, \varepsilon \in\left[0, \varepsilon_{0}\right],
$$

are compact projections on $X_{\varepsilon}^{\alpha}$ and $Q_{\varepsilon}\left(\lambda_{j}^{0}\right) \stackrel{\mathrm{CC}}{\longrightarrow} Q_{0}\left(\lambda_{j}^{0}\right)$. Moreover it is valid the following estimate,

$$
\left\|Q_{\varepsilon}\left(\lambda_{j}^{0}\right) E_{\varepsilon}-E_{\varepsilon} Q_{0}\left(\lambda_{j}^{0}\right)\right\|_{\mathscr{L}\left(X_{0}, X_{\varepsilon}^{\alpha}\right)} \leq C \tau(\varepsilon)
$$

for each $j=1, \cdots, n$, where $C$ is a constant independent of $\varepsilon$.

(ii) All projected spaces $W_{\varepsilon}\left(\lambda_{j}^{0}\right)=Q_{\varepsilon}\left(\lambda_{j}^{0}\right) X_{\varepsilon}^{\alpha}$ have the same dimension, in fact, for $j=$ $1, \ldots, n$, we have

$$
\operatorname{rank}\left(Q_{\varepsilon}\left(\lambda_{j}^{0}\right)\right)=\operatorname{dim}\left(W_{\varepsilon}\left(\lambda_{j}^{0}\right)\right)=\operatorname{dim}\left(W_{0}\left(\lambda_{j}^{0}\right)\right)=\operatorname{rank}\left(Q_{0}\left(\lambda_{j}^{0}\right)\right), \forall \varepsilon \in\left(0, \varepsilon_{0}\right]
$$

(iii) For each $u^{0} \in W_{0}\left(\lambda_{j}^{0}\right)$ there is a sequence $\left\{u^{\varepsilon_{k}}\right\}$ such that $u^{\varepsilon_{k}} \in W_{\varepsilon}\left(\lambda_{j}^{0}\right), \varepsilon_{k} \rightarrow 0$ and $u^{\varepsilon_{k}} \stackrel{\mathrm{E}}{\longrightarrow} u^{0}$.

(iv) Given sequences $\varepsilon_{k}$ and $\left\{u^{\varepsilon_{k}}\right\}$ such that $u^{\varepsilon_{k}} \in W_{\varepsilon}\left(\lambda_{j}^{0}\right)$ and $\left\|u^{\varepsilon_{k}}\right\|_{X_{\varepsilon_{k}}^{\alpha}}=1$ for all $k \in \mathbb{N}$, each subsequence $\left\{u^{\varepsilon_{k_{l}}}\right\}$ of $\left\{u^{\varepsilon_{k}}\right\}$ has a subsequence that converges to some $u^{0} \in W_{0}\left(\lambda_{j}^{0}\right)$. 
Proof. (i) Since $A_{\varepsilon}^{-1}$ is compact we have $Q_{\varepsilon}\left(\lambda_{j}^{0}\right)$ compact projection with finite rank (see (KATO, 1980)). By assumption $A_{\varepsilon}^{-1} \stackrel{C C}{\longrightarrow} A_{0}^{-1}$ which implies $Q_{\varepsilon}\left(\lambda_{j}^{0}\right) \stackrel{\mathrm{CC}}{\longrightarrow} Q_{0}\left(\lambda_{j}^{0}\right)$. Note that for appropriated $\delta>0$ we have

$$
Q_{\varepsilon}\left(\lambda_{j}^{0}\right) E_{\varepsilon}-E_{\varepsilon} Q_{0}\left(\lambda_{j}^{0}\right)=\frac{1}{2 \pi i} \int_{\left|z+\lambda_{j}^{0}\right|=\delta}\left(z+A_{\varepsilon}\right)^{-1} E_{\varepsilon}-E_{\varepsilon}\left(z+A_{0}\right)^{-1} d z
$$

and since $M_{\varepsilon} E_{\varepsilon}=I_{X_{0}}$, using (3.9) we obtain (3.10).

(ii) If $v_{1}, \ldots, v_{l}$ is a basis for $W_{0}\left(\lambda_{j}^{0}\right)$, then for suitably small $\varepsilon$, the set

$$
\left\{Q_{\varepsilon}\left(\lambda_{j}^{0}\right) E_{\varepsilon} v_{1}, \ldots, Q_{\varepsilon}\left(\lambda_{j}^{0}\right) E_{\varepsilon} v_{l}\right\}
$$

is linearly independent in $W_{\varepsilon}\left(\lambda_{j}^{0}\right)$. Thus $\operatorname{dim}\left(W_{\varepsilon}\left(\lambda_{j}^{0}\right)\right) \geq \operatorname{dim}\left(W_{0}\left(\lambda_{j}^{0}\right)\right)$. To prove the equality, suppose that for some sequence $\varepsilon_{k} \rightarrow 0, \operatorname{dim}\left(W_{\varepsilon}\left(\lambda_{j}^{0}\right)\right)>\operatorname{dim}\left(W_{0}\left(\lambda_{j}^{0}\right)\right)$. From Lemma IV.2.3 in (KATO, 1980), for each $k \in \mathbb{N}$, there is a $u^{\varepsilon_{k}} \in W_{\varepsilon_{k}}\left(\lambda_{j}^{0}\right)$ with $\left\|u^{\varepsilon_{k}}\right\|_{X_{\varepsilon_{k}}^{\alpha}}=1$ such that $\operatorname{dist}_{\varepsilon}\left(u^{\varepsilon_{k}}, E_{\varepsilon_{k}} W_{0}\left(\lambda_{j}^{0}\right)\right)=1$, thus by item (i) above we may assume that $u^{\varepsilon_{k}}=Q_{\varepsilon_{k}} u^{\varepsilon_{k}} \stackrel{E}{\longrightarrow}$ $Q_{0}\left(\lambda_{j}^{0}\right)=u^{0}$. Thus,

$$
1 \leq\left\|u^{\varepsilon_{k}}-E_{\varepsilon} Q_{0}\left(\lambda_{j}^{0}\right) u^{0}\right\|_{X_{\varepsilon_{k}}^{\alpha}}=\left\|Q_{\varepsilon}\left(\lambda_{j}^{0}\right) u^{\varepsilon_{k}}-E_{\varepsilon} Q_{0}\left(\lambda_{j}^{0}\right) u^{0}\right\|_{X_{\varepsilon_{k}}^{\alpha}} \stackrel{\varepsilon \rightarrow 0}{\longrightarrow} 0,
$$

which is a contradiction.

(iii) If $\varepsilon_{k} \rightarrow 0$ then $E_{\varepsilon_{k}} u^{0} \stackrel{E}{\longrightarrow} u^{0}$ and then $u^{\varepsilon_{k}}=Q_{\varepsilon_{k}}\left(\lambda_{j}^{0}\right) E_{\varepsilon} u^{0} \stackrel{E}{\longrightarrow} Q_{0}\left(\lambda_{j}^{0}\right) u^{0}$. Since $Q_{0}\left(\lambda_{j}^{0}\right)$ is a projection and $u^{0} \in W_{0}\left(\lambda_{j}^{0}\right)$ we have $Q_{0}\left(\lambda_{j}^{0}\right) u^{0}=u^{0}$, which proves the result.

(iv) Since $Q_{\varepsilon_{k}}\left(\lambda_{j}^{0}\right)$ is a projection we have $Q_{\varepsilon_{k}} u^{\varepsilon_{k}}=u^{\varepsilon_{k}}$ and follows from item (i) above that

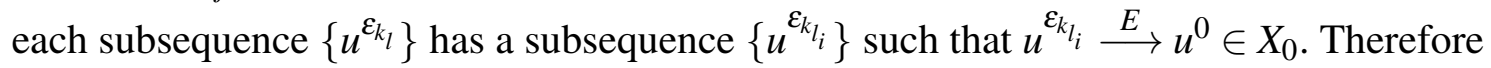

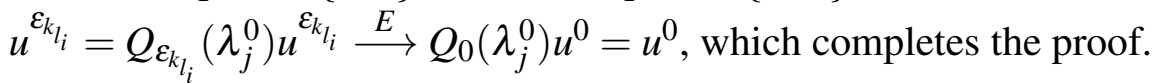

Remark 3.2.7. If we choose $\delta>0$ such that $\left\{\lambda \in \mathbb{C}:\left|\lambda+\lambda_{j}^{0}\right|=\delta\right\} \cap \sigma\left(A_{\varepsilon}\right)$ is equal the eigenvalues $\lambda_{1}^{\varepsilon}, \ldots, \lambda_{j}^{\varepsilon}$, then $Q_{\varepsilon}\left(\lambda_{j}^{0}\right)=Q_{\varepsilon}^{j}$, where $Q_{\varepsilon}^{j}$ is the orthogonal projection onto $\operatorname{span}\left[\varphi_{1}^{\varepsilon}, \ldots, \varphi_{j}^{\varepsilon}\right]$ considered in the previous section.

As a consequence of Proposition 3.2.6 we have the following result.

Corollary 3.2.8. The following convergences hold,

(i) $\lambda_{j}^{\varepsilon} \rightarrow \infty$ as $\varepsilon \rightarrow 0, j \geq n+1$ (Gap condition).

(ii) $\left|\lambda_{j}^{\varepsilon}-\lambda_{j}^{0}\right| \leq C \tau(\varepsilon), j=1, \cdots, n$, where $C$ is a constant independent of $\varepsilon$. 
Proof. We start by asserting that $\lambda_{j}^{\varepsilon} \rightarrow \lambda_{j}^{0}$ as $\varepsilon \rightarrow 0$ for $j=1, \ldots, n$. If this is not the case then there are $\varepsilon>0$ and sequence $\varepsilon_{k} \rightarrow 0$ such that

$$
\int_{\left|\lambda-\lambda_{j}^{0}\right|=\delta}\left(\lambda-\lambda_{j}^{0}\right)^{k}\left(\lambda-A_{\varepsilon_{k}}\right)^{-1} d \lambda=0, \quad \forall k \in \mathbb{N} .
$$

Since $A_{\varepsilon}^{-1}$ converges compactly to $A_{0}^{-1}$, we have

$$
\int_{\left|\lambda-\lambda_{j}^{0}\right|=\delta}\left(\lambda-\lambda_{j}^{0}\right)^{k}\left(\lambda-A_{0}\right)^{-1} d \lambda=0, \quad \forall k \in \mathbb{N}
$$

which is an absurd because since $\lambda_{j}^{0}$ is an eigenvalue of $A_{0},\left(\lambda-A_{0}\right)^{-1}$ doesn't have removable singularity at $\lambda=\lambda_{j}^{0}$.

If (i) fails, we can take $R>0$ and sequences $\varepsilon_{k} \rightarrow \infty$ and $\left\{\lambda_{j}^{\varepsilon_{k}}\right\}, j \geq n+1$ such that $\left|\lambda_{j}^{\varepsilon_{k}}\right| \leq R$. We can assume $\lambda_{j}^{\varepsilon_{k}} \rightarrow \lambda$. Let $\varphi_{j}^{\varepsilon_{k}}$ be the corresponding eigenfunction to $\lambda_{j}^{\varepsilon_{k}}$ with

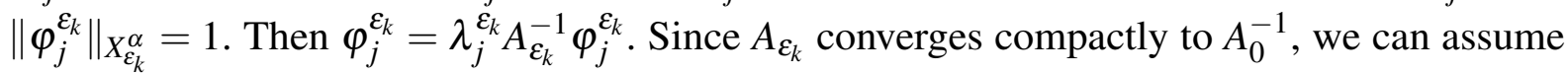
$\varphi_{j}^{\varepsilon_{k}} \rightarrow u^{0}$ as $\varepsilon_{k} \rightarrow 0$ for some $u^{0} \in X_{0}$. Thus

$$
\varphi_{j}^{\varepsilon_{k}}=\lambda_{j}^{\varepsilon_{k}} A_{\varepsilon_{k}}^{-1} \varphi_{j}^{\varepsilon_{k}} \rightarrow \lambda A_{0}^{-1} u^{0}
$$

as $\varepsilon_{k} \rightarrow 0$. Since $\varphi_{j}^{\varepsilon_{k}} \rightarrow u^{0}$, we get $u^{0}=\lambda A_{0}^{-1} u^{0}$, which implies $\lambda \in \sigma\left(A_{0}\right)$, thus $\lambda=\lambda_{j}^{0}$, for some $j=1, \ldots, n$, and $\lambda_{j}^{\varepsilon_{k}} \rightarrow \lambda_{j}^{0}$ as $\varepsilon_{k} \rightarrow 0, j \geq n+1$, which is an absurd.

Now we prove (ii). Since $\lambda_{j}^{\varepsilon} \stackrel{\varepsilon \rightarrow 0}{\longrightarrow} \lambda_{j}^{0}$, for each $j=1, \ldots, n$, then for $\varepsilon$ sufficiently small there is $u^{0} \in \operatorname{Kernel}\left(\lambda_{j}^{0}-A_{0}\right)$ with $\left\|E_{\varepsilon} u^{0}\right\|_{X_{\varepsilon}^{\alpha}}=1$ such that $Q_{\varepsilon}\left(\lambda_{j}^{0}\right) E_{\varepsilon} u^{0}$ is eigenvalue of $A_{\varepsilon}$ associated with $\lambda_{j}^{\varepsilon}$, thus

$$
\begin{aligned}
\left|\lambda_{j}^{\varepsilon}-\lambda_{j}^{0}\right| & \leq\left|\lambda_{j}^{\varepsilon} \lambda_{j}^{0}\right|\left\|E_{\varepsilon} A_{0}^{-1} Q_{0}\left(\lambda_{j}^{0}\right) u^{0}-A_{\varepsilon}^{-1} Q_{\varepsilon}\left(\lambda_{j}^{0}\right) E_{\varepsilon} u^{0}\right\|_{X_{\varepsilon}^{\alpha}} \\
& +\left|\lambda_{j}^{0}\right|\left\|\left(Q_{\varepsilon}\left(\lambda_{j}^{0}\right) E_{\varepsilon}-E_{\varepsilon} Q_{0}\left(\lambda_{j}^{0}\right)\right) u^{0}\right\|_{X_{\varepsilon}^{\alpha}},
\end{aligned}
$$

and

$$
\begin{aligned}
\left\|E_{\varepsilon} A_{0}^{-1} Q_{0}\left(\lambda_{j}^{0}\right) u^{0}-A_{\varepsilon}^{-1} Q_{\varepsilon}\left(\lambda_{j}^{0}\right) E_{\varepsilon} u^{0}\right\|_{X_{\varepsilon}^{\alpha}} & \leq\left\|\left(E_{\varepsilon} A_{0}^{-1} M_{\varepsilon}-A_{\varepsilon}^{-1}\right) E_{\varepsilon} Q_{0}\left(\lambda_{j}^{0}\right) u^{0}\right\|_{X_{\varepsilon}^{\alpha}} \\
& +\left\|A_{\varepsilon}^{-1}\left(E_{\varepsilon} Q_{0}\left(\lambda_{j}^{0}\right)-Q_{\varepsilon}\left(\lambda_{j}^{0}\right) E_{\varepsilon}\right) u^{0}\right\|_{X_{\varepsilon}^{\alpha}}
\end{aligned}
$$

The result follows from (3.7) and (3.10).

Since we have the gap condition $\lambda_{j}^{\varepsilon} \rightarrow \infty$, as $\varepsilon \rightarrow 0$ for $j \geq n+1$ proved in Corollary 3.2.8, we can take $\delta>0$ and construct, for $\varepsilon$ sufficiently small (we still denote $\varepsilon \in\left(0, \varepsilon_{0}\right]$ ), the curve $\bar{\Gamma}=\Gamma_{1}+\Gamma_{2}+\Gamma_{3}+\Gamma_{4} \subset \rho\left(-A_{\varepsilon}\right)$, where

$$
\begin{gathered}
\Gamma_{1}=\left\{\mu \in \mathbb{C} ; \operatorname{Re}(\mu)=-\lambda_{1}^{0}+\delta \text { and }|\operatorname{Im}(\mu)| \leq 1\right\}, \\
\Gamma_{2}=\left\{\mu \in \mathbb{C} ;-\lambda_{n}^{0}-\delta \leq \operatorname{Re}(\mu) \leq-\lambda_{0}^{1}+\delta \text { and } \operatorname{Im}(\mu)=1\right\}, \\
\Gamma_{3}=\left\{\mu \in \mathbb{C} ; \operatorname{Re}(\mu)=-\lambda_{n}^{0}-\delta \text { and }|\operatorname{Im}(\mu)| \leq 1\right\},
\end{gathered}
$$




$$
\Gamma_{4}=\left\{\mu \in \mathbb{C} ;-\lambda_{n}^{0}-\delta \leq \operatorname{Re}(\mu) \leq-\lambda_{1}^{0}+\delta \text { and } \operatorname{Im}(\mu)=-1\right\} .
$$

For $\varepsilon \in\left[0, \varepsilon_{0}\right]$ we define the spectral projection

$$
Q_{\varepsilon}=\frac{1}{2 \pi i} \int_{\bar{\Gamma}}\left(\lambda+A_{\varepsilon}\right)^{-1} d \lambda
$$

Note that $Q_{0}=I_{X_{0}}$ and $Q_{\varepsilon}$ coincides with the spectral projection $Q_{n}^{\varepsilon}$ previously considered and thus, the eigenspace $Q_{\varepsilon} X_{\varepsilon}^{\alpha}=\operatorname{span}\left[\varphi_{1}^{\varepsilon}, \cdots, \varphi_{n}^{\varepsilon}\right]$ is isomorphic to $\mathbb{R}^{n}$. Similarly the proof of Lemma 3.2.6 we obtain the following result.

Lemma 3.2.9. Let $Q_{\varepsilon}$ be the spectral projection defined in (3.11). There is a constant $C$ independent of $\varepsilon$ such that the following properties are valid,

(i) $\left\|Q_{\varepsilon}\right\|_{\mathscr{L}\left(X_{\varepsilon}, X_{\varepsilon}^{\alpha}\right)} \leq C$;

(ii) $\left\|Q_{\varepsilon} E_{\varepsilon}-E_{\varepsilon}\right\|_{\mathscr{L}\left(X_{0} X_{\varepsilon}^{\alpha}\right)} \leq C \tau(\varepsilon)$.

The next result play an important role in the existence of invariant manifold proved in the next section.

Lemma 3.2.10. Let $Q_{\varepsilon}$ be the spectral projection defined in (3.11). If we denote, for each $\varepsilon \in\left(0, \varepsilon_{0}\right], Y_{\varepsilon}=Q_{\varepsilon} X_{\varepsilon}^{\alpha}$ and $Z_{\varepsilon}=\left(I-Q_{\varepsilon}\right) X_{\varepsilon}^{\alpha}$ and define the projected operators

$$
A_{\varepsilon}^{+}=\left.A_{\varepsilon}\right|_{Y_{\varepsilon}} \quad \text { and } \quad A_{\varepsilon}^{-}=\left.A_{\varepsilon}\right|_{Z_{\varepsilon}}
$$

then $-A_{\varepsilon}^{-}$generates a semigroup in $Z_{\varepsilon},-A_{\varepsilon}^{+}$generates a group in $Y_{\varepsilon}$ and the following estimates are valid,

(i) $\left\|e^{-A_{\varepsilon}^{-} t} z\right\|_{X_{\varepsilon}^{\alpha}} \leq M e^{-\beta t}\|z\|_{X_{\varepsilon}^{\alpha}}, z \in Z_{\varepsilon}, t>0$;

(ii) $\left\|e^{-A_{\varepsilon}^{-} t} z\right\|_{X_{\varepsilon}^{\alpha}} \leq M e^{-\beta t} t^{-\alpha}\|z\|_{X_{\varepsilon}}, z \in Z_{\varepsilon}, t>0$;

(iii) $\left\|e^{-A_{\varepsilon}^{+} t} z\right\|_{X_{\varepsilon}^{\alpha}} \leq M e^{-\bar{\gamma} t}\|z\|_{X_{\varepsilon}^{\alpha}}, z \in Y_{\varepsilon}, t>0$;

(iv) $\left\|e^{-A_{\varepsilon}^{+} t} z\right\|_{X_{\varepsilon}^{\alpha}} \leq M e^{-\gamma t}\|z\|_{X_{\varepsilon}^{\alpha}}, z \in Y_{\varepsilon}, t<0$;

(v) $\left\|E_{\varepsilon} e^{-A_{0} t} M_{\varepsilon} z\right\|_{X_{\varepsilon}^{\alpha}} \leq M e^{-\gamma t}\|z\|_{X_{\varepsilon}^{\alpha}}, z \in X_{\varepsilon}^{\alpha}, t<0$;

(vi) $\left\|e^{-A_{\varepsilon}^{+} t} z-E_{\varepsilon} e^{-A_{0} t} M_{\varepsilon} z\right\|_{X_{\varepsilon}^{\alpha}} \leq M e^{-\gamma t} \tau(\varepsilon)\|z\|_{X_{\varepsilon}^{\alpha}}, z \in Y_{\varepsilon}, t<0$;

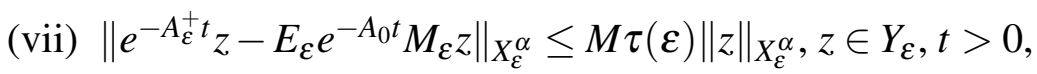

where $\beta=\lambda_{n+1}^{\varepsilon} \rightarrow \infty$ as $\varepsilon \rightarrow 0, \bar{\gamma}=\lambda_{1}^{\varepsilon}, \gamma=\lambda_{n}^{0}+\delta$ and $M$ is a constant independent of $\varepsilon$. 
Proof. (i) We have for $z \in Z_{\varepsilon}$,

$$
e^{-A_{\varepsilon}^{-} t} z=\sum_{i=n+1}^{\infty} e^{-\lambda_{i}^{\varepsilon} t}\left\langle z, \varphi_{i}^{\varepsilon}\right\rangle_{X_{\varepsilon}} \varphi_{i}^{\varepsilon}, \quad t>0,
$$

but if $i \geq n+1$, then $\lambda_{n+1}^{\varepsilon} \leq \lambda_{i}^{\varepsilon}$ which implies $e^{-\lambda_{i}^{\varepsilon} t} \leq e^{\lambda_{n+1}^{\varepsilon} t}$ for $t>0$. Thus

$$
\left\|e^{-A_{\varepsilon}^{-} t} z\right\|_{X_{\varepsilon}^{\alpha}} \leq\left(e^{-2 \lambda_{n+1}^{\varepsilon} t} \sum_{i=n+1}^{\infty}\left\langle z, \varphi_{i}^{\varepsilon}\right\rangle_{X_{\varepsilon}}^{2}\left(\lambda_{i}^{\varepsilon}\right)^{2 \alpha}\right)^{\frac{1}{2}} \leq M e^{-\lambda_{n+1}^{\varepsilon} t}\|z\|_{X_{\varepsilon}^{\alpha}}, \quad t>0 .
$$

(ii) The function $f(\mu)=e^{-\mu t} \mu^{\alpha}$ attains its maximum at $\mu=\alpha / t, t>0$. Then,

$$
\left\|e^{-A_{\varepsilon}^{-} t} z\right\|_{X_{\varepsilon}^{\alpha}} \leq\left\{\begin{array}{l}
e^{-\lambda_{n+1}^{\varepsilon} t}\left(\lambda_{n+1}^{\varepsilon}\right)^{\alpha}\|z\|_{X_{\varepsilon}}, \alpha / t<\lambda_{n+1}^{\varepsilon} \\
e^{-\lambda_{n+1}^{\varepsilon} t}(\alpha / t)^{\alpha}\|z\|_{X_{\varepsilon}}, \alpha / t>\lambda_{n+1}^{\varepsilon}
\end{array}\right.
$$

(iii) We have for $z \in Y_{\varepsilon}$,

$$
e^{-A_{\varepsilon}^{+} t} z=\sum_{i=1}^{n} e^{-\lambda_{i}^{\varepsilon} t}\left\langle z, \varphi_{i}^{\varepsilon}\right\rangle_{X_{\varepsilon}} \varphi_{i}^{\varepsilon},
$$

but if $i \leq n+1$, then $\lambda_{1}^{\varepsilon} \leq \lambda_{i}^{\varepsilon}$ which implies $e^{-\lambda_{i}^{\varepsilon} t} \leq e^{-\lambda_{1}^{\varepsilon} t}$ for $t>0$. Thus $\left\|e^{-A_{\varepsilon}^{+} t} z\right\|_{X_{\varepsilon}^{\alpha}} \leq$ $M e^{-\lambda_{1}^{\varepsilon} t}\|z\|_{X_{\varepsilon}^{\alpha}}, t>0$.

(iv) Similarly to item (iii) we obtain $\left\|e^{-A_{\varepsilon}^{+} t} z\right\|_{X_{\varepsilon}^{\alpha}} \leq M e^{-\lambda_{n}^{\varepsilon} t}\|z\|_{X_{\varepsilon}^{\alpha}}, t<0$. But $\lambda_{n}^{\varepsilon} \rightarrow \lambda_{n}^{0}<\lambda_{n}^{0}+\delta$, thus we can take $\varepsilon$ sufficiently small such that $e^{-\lambda_{n}^{\varepsilon}} \leq e^{-\left(\lambda_{n}^{0}+\delta\right) t}, t<0$.

(v) Similarly to item (iii) we also obtain $\left\|E_{\varepsilon} e^{-A_{0} t} M_{\varepsilon} z\right\|_{X_{\varepsilon}^{\alpha}} \leq M e^{-\left(\lambda_{n}^{0}+\delta\right) t}\|z\|_{X_{\varepsilon}^{\alpha}}, t<0$, where we have used that $E_{\varepsilon}$ and $M_{\varepsilon}$ are uniformly bounded.

(vi) Since $A_{\mathcal{\varepsilon}}^{+}$is a bounded operator in the finite dimensional space, for $z \in Y_{\varepsilon}$ and $t<0$, we have

$$
\begin{aligned}
\left\|e^{-A_{\varepsilon}^{+} t} z-E_{\varepsilon} e^{-A_{0} t} M_{\varepsilon} z\right\|_{X_{\varepsilon}^{\alpha}} & \leq \frac{1}{2 \pi} \int_{\bar{\Gamma}}\left\|e^{\mu t}\left(\left(\mu+A_{\varepsilon}\right)^{-1} z-E_{\varepsilon}\left(\mu+A_{0}\right)^{-1} M_{\varepsilon} z\right)\right\|_{X_{\varepsilon}^{\alpha}} d \mu \\
& \leq C \tau(\varepsilon) \int_{\bar{\Gamma}}\left|e^{\mu t}\right||d \mu|\|z\|_{X_{\varepsilon}^{\alpha}} \leq C \tau(\varepsilon) \sup _{\mu \in \bar{\Gamma}} e^{\operatorname{Re}(\mu) t}\|z\|_{X_{\varepsilon}^{\alpha}} \\
& \leq M e^{-\left(\lambda_{n}^{0}+\delta\right) t} \tau(\varepsilon)\|z\|_{X_{\varepsilon}^{\alpha}},
\end{aligned}
$$

where we have used $X_{\mathcal{\varepsilon}}^{\alpha} \subset X_{\mathcal{\varepsilon}}$ uniformly.

(vii) Analogous to item (vi) using that $\bar{\Gamma}$ is compact and $e^{\mu t}$ is continuous.

\subsection{Invariant Manifold}

In this section we use the gap condition in the Corollary 3.2.8 and the estimates in the Lemma 3.2.10 to construct an invariant manifolds for the problems (3.2). We prove that these manifolds converge to $X_{0}$ as $\varepsilon \rightarrow 0$ and this convergence can be estimate by $\tau(\varepsilon)+\rho(\varepsilon)$. 
Theorem 3.3.1. For $\varepsilon$ sufficiently small there is an invariant manifold $\mathscr{M}_{\varepsilon}$ for (3.2), which is given by graph of a certain Lipschitz continuous map $s_{*}^{\varepsilon}: Y_{\varepsilon} \rightarrow Z_{\varepsilon}$ as

$$
\mathscr{M}_{\varepsilon}=\left\{u^{\varepsilon} \in X_{\varepsilon}^{\alpha} ; u^{\varepsilon}=Q_{\varepsilon} u^{\varepsilon}+s_{*}^{\varepsilon}\left(Q_{\varepsilon} u^{\varepsilon}\right)\right\}
$$

The $\operatorname{map} s_{*}^{\varepsilon}: Y_{\varepsilon} \rightarrow Z_{\varepsilon}$ satisfies the condition

$$
\left\|s_{*}^{\varepsilon}\right\|\left\|=\sup _{v^{\varepsilon} \in Y_{\varepsilon}}\right\| s_{*}^{\varepsilon}\left(v^{\varepsilon}\right) \|_{X_{\varepsilon}^{\alpha}} \leq C(\tau(\varepsilon)+\rho(\varepsilon)),
$$

for some positive constant $C$ independent of $\varepsilon$. The invariant manifold $\mathscr{M}_{\varepsilon}$ is exponentially attracting and the global attractor $\mathscr{A}_{\varepsilon}$ of the problem (3.2) lying in $\mathscr{M}_{\varepsilon}$ and the flow on $\mathscr{A}_{\varepsilon}$ is given by

$$
u^{\varepsilon}(t)=v^{\varepsilon}(t)+s_{*}^{\varepsilon}\left(v^{\varepsilon}(t)\right), \quad t \in \mathbb{R},
$$

where $v^{\varepsilon}(t)$ satisfies

$$
\dot{v}^{\varepsilon}+A_{\varepsilon}^{+} v^{\varepsilon}=Q_{\varepsilon} f_{\varepsilon}\left(v^{\varepsilon}+s_{*}^{\varepsilon}\left(v^{\varepsilon}(t)\right)\right)
$$

Proof. Given $L, \Delta>0$ we consider the set

$$
\Sigma_{\varepsilon}=\left\{s^{\varepsilon}: Y_{\varepsilon} \rightarrow Z_{\varepsilon} ;\left\|s^{\varepsilon}\right\| \mid \leq D \text { and }\left\|s^{\varepsilon}(v)-s^{\varepsilon}(\tilde{v})\right\|_{X_{\varepsilon}^{\alpha}} \leq \Delta\|v-\tilde{v}\|_{Y_{\varepsilon}}\right\}
$$

Thus $\left(\Sigma_{\varepsilon},\|\cdot\| \|\right)$ is a complete metric space. We write the solution $u^{\varepsilon}$ of (3.2) as $u^{\varepsilon}=v^{\varepsilon}+z^{\varepsilon}$, with $v^{\varepsilon} \in Y_{\varepsilon}$ and $z^{\varepsilon} \in Z_{\varepsilon}$ and since $Q_{\varepsilon}$ and $I-Q_{\varepsilon}$ commute with $A_{\varepsilon}$, we can write (3.2) in the coupled form

$$
\left\{\begin{array}{l}
v_{t}^{\varepsilon}+A_{\varepsilon}^{+} v^{\varepsilon}=Q_{\varepsilon} f_{\varepsilon}\left(v^{\varepsilon}+z^{\varepsilon}\right):=H_{\varepsilon}\left(v^{\varepsilon}, z^{\varepsilon}\right) \\
z_{t}^{\varepsilon}+A_{\varepsilon}^{-} z^{\varepsilon}=\left(I-Q_{\varepsilon}\right) f_{\varepsilon}\left(v^{\varepsilon}+z^{\varepsilon}\right):=G_{\varepsilon}\left(v^{\varepsilon}, z^{\varepsilon}\right) .
\end{array}\right.
$$

Since $f_{\varepsilon}$ is continuously differentiable we can choose $\rho>0$ such that for all $v^{\varepsilon}, \tilde{v}^{\varepsilon} \in Y_{\varepsilon}$ and $z^{\varepsilon}, \tilde{z}^{\varepsilon} \in Z_{\varepsilon}$, we have

$$
\begin{aligned}
& \left\|H_{\varepsilon}\left(v^{\varepsilon}, z^{\varepsilon}\right)\right\|_{X_{\varepsilon}^{\alpha}} \leq \rho, \quad\left\|G_{\varepsilon}\left(v^{\varepsilon}, z^{\varepsilon}\right)\right\|_{X_{\varepsilon}^{\alpha}} \leq \rho \\
& \left\|H_{\varepsilon}\left(v^{\varepsilon}, z^{\varepsilon}\right)-H_{\varepsilon}\left(\tilde{v}^{\varepsilon}, \tilde{z}^{\varepsilon}\right)\right\|_{X_{\varepsilon}^{\alpha}} \leq \rho\left(\left\|v^{\varepsilon}-\tilde{v}_{\varepsilon}\right\|_{X_{\varepsilon}^{\alpha}}+\left\|z^{\varepsilon}-\tilde{z}_{\varepsilon}\right\|_{X_{\varepsilon}^{\alpha}}\right), \\
& \left\|G_{\varepsilon}\left(v^{\varepsilon}, z^{\varepsilon}\right)-G_{\varepsilon}\left(\tilde{v}^{\varepsilon}, \tilde{z}^{\varepsilon}\right)\right\|_{X_{\varepsilon}^{\alpha}} \leq \rho\left(\left\|v^{\varepsilon}-\tilde{v}_{\varepsilon}\right\|_{X_{\varepsilon}^{\alpha}}+\left\|z^{\varepsilon}-\tilde{z}_{\varepsilon}\right\|_{X_{\varepsilon}^{\alpha}}\right),
\end{aligned}
$$

and for $\varepsilon$ sufficiently small, we can take

$$
\begin{aligned}
& \rho M \beta^{-1} \leq D, \quad 0 \leq \beta-\gamma-\rho M(1+\Delta), \quad 0 \leq \beta-\gamma-\rho M \\
& \frac{\rho M^{2}(1+\Delta)}{\beta-\gamma-\rho M(1+\Delta)} \leq \Delta, \quad \rho M \beta^{-1}+\frac{\rho^{2} M^{2}(1+\Delta) \gamma^{-1}}{\beta-\gamma-\rho M(1+\Delta)} \leq \frac{1}{2} \\
& \rho M \beta^{-1}+\frac{\rho^{2} M^{2} \gamma^{-1}}{\beta-\gamma-\rho M}<1, \quad L=\left[\rho M+\frac{\rho^{2} M^{2}(1+\Delta)(1+M)}{\beta-\gamma-\rho M(1+\Delta)}\right], \beta-L>0,
\end{aligned}
$$

where $\beta, \gamma$ and $M$ are given in the Lemma 3.2.10. Note that, since we have the gap condition $\beta=\beta(\varepsilon)=\lambda_{n+1}^{\varepsilon} \rightarrow \infty$ as $n \rightarrow \infty$, the estimates (3.15) are satisfied. 
Let $s^{\varepsilon} \in \Sigma_{\varepsilon}$ and $v^{\varepsilon}(t)=v^{\varepsilon}\left(t, \tau, \eta, s^{\varepsilon}\right)$ be the solution of

$$
\left\{\begin{array}{l}
v_{t}^{\varepsilon}+A_{\varepsilon}^{+} v^{\varepsilon}=H_{\mathcal{\varepsilon}}\left(v^{\varepsilon}, s^{\varepsilon}\left(v^{\varepsilon}\right)\right), \quad t<\tau \\
v^{\varepsilon}(\tau)=\eta \in Y_{\varepsilon}
\end{array}\right.
$$

We define $\Phi_{\varepsilon}: \Sigma_{\varepsilon} \rightarrow \Sigma_{\varepsilon}$ given by

$$
\Phi_{\varepsilon}\left(s^{\varepsilon}\right)(\eta)=\int_{-\infty}^{\tau} e^{-A_{\varepsilon}^{-}(\tau-r)} G_{\varepsilon}\left(v^{\varepsilon}(r), s^{\varepsilon}\left(v^{\varepsilon}(r)\right)\right) d r .
$$

Note that $G_{\varepsilon}$ and $H_{\varepsilon}$ are maps acting in $X_{\mathcal{\varepsilon}}^{\alpha}$, then by (3.14), (3.15) and Lemma 3.2.10, we have

$$
\left\|\Phi_{\varepsilon}\left(s^{\varepsilon}\right)(\eta)\right\|_{X_{\varepsilon}^{\alpha}} \leq \rho M \int_{-\infty}^{\tau} e^{-\beta(\tau-r)} d r=\rho M \beta^{-1} \leq D
$$

Also for $s^{\varepsilon}, \tilde{s}^{\varepsilon} \in \Sigma_{\varepsilon}, \eta, \tilde{\eta} \in Y_{\varepsilon}, v^{\varepsilon}(t)=v^{\varepsilon}\left(t, \tau, \eta, s^{\varepsilon}\right)$ and $\tilde{v}^{\varepsilon}(t)=\tilde{v}^{\varepsilon}\left(t, \tau, \tilde{\eta}, \tilde{s}^{\varepsilon}\right)$ we have

$$
\begin{aligned}
v^{\varepsilon}(t)-\tilde{v}^{\varepsilon}(t)=e^{-A_{\varepsilon}^{+}(t-\tau)}(\eta & -\tilde{\eta}) \\
& +\int_{\tau}^{t} e^{-A_{\varepsilon}^{+}(t-r)}\left[H_{\mathcal{\varepsilon}}\left(v^{\varepsilon}(r), s^{\varepsilon}\left(v^{\varepsilon}(r)\right)\right)-H_{\mathcal{\varepsilon}}\left(\tilde{v}^{\varepsilon}(r), \tilde{s}^{\varepsilon}\left(\tilde{v}^{\varepsilon}(r)\right)\right)\right] d r,
\end{aligned}
$$

and we can prove using Gronwall's inequality that

$$
\left\|v^{\varepsilon}(t)-\tilde{v}^{\varepsilon}(t)\right\|_{X_{\varepsilon}^{\alpha}} \leq\left[M\|\eta-\tilde{\eta}\|_{X_{\varepsilon}^{\alpha}}+\rho M \gamma^{-1}\left\|s^{\varepsilon}-\tilde{s}^{\varepsilon}\right\|\right] e^{[\rho M(1+\Delta)+\gamma](\tau-t)} .
$$

From this we obtain

$$
\begin{aligned}
& \left\|\Phi_{\varepsilon}\left(s^{\varepsilon}\right)(\eta)-\Phi_{\varepsilon}\left(\tilde{s}^{\varepsilon}\right)(\tilde{\eta})\right\|_{X_{\varepsilon}^{\alpha}} \\
& \quad \leq\left[\frac{\rho M^{2}(1+\Delta)}{\beta-\gamma-\rho M(1+\Delta)}\right]\|\eta-\tilde{\eta}\|_{X_{\varepsilon}^{\alpha}}+\left[\rho M \beta^{-1}+\frac{\rho^{2} M^{2}(1+\Delta) \gamma^{-1}}{\beta-\gamma-\rho M(1+\Delta)}\right]\left\|s^{\varepsilon}-\tilde{s}^{\varepsilon}\right\| .
\end{aligned}
$$

Therefore $\Phi_{\varepsilon}$ is a contraction on $\Sigma_{\varepsilon}$ and there is a unique $s_{*}^{\varepsilon} \in \Sigma_{\varepsilon}$.

Let $\left(\bar{v}^{\varepsilon}, \bar{z}^{\varepsilon}\right) \in \mathscr{M}_{\varepsilon}, \bar{z}^{\varepsilon}=s_{*}^{\varepsilon}\left(\bar{v}^{\varepsilon}\right)$ and let $v_{s_{*}}^{\varepsilon}(t)$ be the solution of

$$
\left\{\begin{array}{l}
v_{t}^{\varepsilon}+A_{\varepsilon}^{+} v^{\varepsilon}=H_{\varepsilon}\left(v^{\varepsilon}, s_{*}^{\varepsilon}\left(v^{\varepsilon}\right)\right), \quad t<\tau \\
v^{\varepsilon}(0)=\bar{v}^{\varepsilon}
\end{array}\right.
$$

Thus, $\left\{\left(v_{s_{*}}^{\varepsilon}(t), s_{*}^{\varepsilon}\left(v_{s_{*}}^{\varepsilon}(t)\right)\right\}_{t \in \mathbb{R}}\right.$ defines a curve on $\mathscr{M}_{\varepsilon}$. But the only solution of equation

$$
z_{t}^{\varepsilon}+A_{\varepsilon}^{-} z^{\varepsilon}=G_{\varepsilon}\left(v_{s_{*}}^{\varepsilon}(t), s_{*}^{\varepsilon}\left(v_{s_{*}}^{\varepsilon}(t)\right)\right)
$$

which stay bounded when $t \rightarrow-\infty$ is given by

$$
z_{s_{*}}^{\varepsilon}=\int_{-\infty}^{t} e^{-A_{\varepsilon}^{-}(t-r)} G_{\varepsilon}\left(v_{s_{*}}^{\varepsilon}(t), s_{*}^{\varepsilon}\left(v_{s_{*}}^{\varepsilon}(t)\right)\right) d r=s_{*}^{\varepsilon}\left(v_{s_{*}}^{\varepsilon}(t)\right) .
$$

Therefore $\left(v_{s_{*}}^{\varepsilon}(t), s_{*}^{\varepsilon}\left(v_{s_{*}}^{\varepsilon}(t)\right)\right.$ is a solution of (3.2) through $\left(\bar{v}^{\varepsilon}, \bar{z}^{\varepsilon}\right)$ and thus $\mathscr{M}_{\varepsilon}$ is an invariant manifold. 
Now we will prove the estimate (3.12). We have $Q_{0}=I_{X_{0}}$ which implies $I-Q_{0}=0$, therefore we can assume $\mathscr{M}_{0}=X_{0}, s_{*}^{0}=0$ and $G_{0}\left(v^{0}, s_{*}^{0}\left(v^{0}\right)\right)=0$, where $v^{0}$ is solution of (3.1) with $v^{0}(0)=M_{\varepsilon} \eta$ and $\|\eta\|_{X_{\varepsilon}^{\alpha}} \leq C$, for some constant $C$ independent of $\varepsilon$. Thus

$$
\begin{aligned}
\left\|s_{*}^{\varepsilon}(\eta)\right\|_{X_{\varepsilon}^{\alpha}} & \leq \int_{-\infty}^{\tau}\left\|e^{-A_{\varepsilon}^{-}(\tau-r)} G_{\mathcal{\varepsilon}}\left(v^{\varepsilon}, s_{*}^{\varepsilon}\left(v^{\varepsilon}\right)\right)\right\|_{X_{\varepsilon}^{\alpha}} d r \\
& \leq \int_{-\infty}^{\tau}\left\|e^{-A_{\varepsilon}^{-}(\tau-r)} G_{\varepsilon}\left(v^{\varepsilon}, s_{*}^{\varepsilon}\left(v^{\varepsilon}\right)\right)-e^{-A_{\varepsilon}^{-}(\tau-r)} G_{\mathcal{\varepsilon}}\left(E_{\varepsilon} v^{0}, 0\right)\right\|_{X_{\varepsilon}^{\alpha}} d r \\
& +\int_{-\infty}^{\tau}\left\|e^{-A_{\varepsilon}^{-}(\tau-r)} G_{\varepsilon}\left(E_{\varepsilon} v^{0}, 0\right)\right\|_{X_{\varepsilon}^{\alpha}} d r .
\end{aligned}
$$

If we denote the last two integrals for $I_{1}$ and $I_{2}$ respectively, with the aid of (3.14), (3.15) and Lemma 3.2.10, we get

$$
\begin{aligned}
I_{1} & \leq \int_{-\infty}^{\tau} M e^{-\beta(\tau-r)} \rho\left[\left\|v^{\varepsilon}-E_{\varepsilon} v^{0}\right\|_{X_{\varepsilon}^{\alpha}}+\left\|s_{*}^{\varepsilon}\right\| \|\right] d r \\
& \leq \rho M \int_{-\infty}^{\tau} e^{-\beta(\tau-r)}\left\|v^{\varepsilon}-E_{\varepsilon} v^{0}\right\|_{X_{\varepsilon}^{\alpha}} d r \\
& +\rho M\left\|s_{*}^{\varepsilon}\right\| \int_{-\infty}^{\tau} e^{-\beta(\tau-r)} d r \\
& =\rho M \int_{-\infty}^{\tau} e^{-\beta(\tau-r)}\left\|v^{\varepsilon}-E_{\varepsilon} v^{0}\right\|_{X_{\varepsilon}^{\alpha}} d r+\rho M \beta^{-1}\left\|s_{*}^{\varepsilon}\right\| .
\end{aligned}
$$

For $I_{2}$, observe that

$$
G_{\varepsilon}\left(E_{\varepsilon} v^{0}, 0\right)=\left(I-Q_{\varepsilon}\right) f_{\varepsilon}\left(E_{\varepsilon} v^{0}\right)=\left(I-Q_{\varepsilon}\right)\left[f_{\varepsilon}\left(E_{\varepsilon} v^{0}\right)-E_{\varepsilon} f_{0}\left(v^{0}\right)\right]+\left(I-Q_{\varepsilon}\right) E_{\varepsilon} f_{0}\left(v^{0}\right),
$$

thus by (3.8) and Lemma (3.2.9), we have $I_{2} \leq C(\tau(\varepsilon)+\rho(\varepsilon))$, for some constant $C$ independent of $\varepsilon$. Therefore

$$
\left\|s_{*}^{\varepsilon}(\eta)\right\|_{X_{\varepsilon}^{\alpha}} \leq C(\tau(\varepsilon)+\rho(\varepsilon))+\rho M \beta^{-1}\left\|s_{*}^{\varepsilon}\right\|\left\|+\rho M \int_{-\infty}^{\tau} e^{-\beta(\tau-r)}\right\| v^{\varepsilon}-E_{\varepsilon} v^{0} \|_{X_{\varepsilon}^{\alpha}} d r .
$$

But, for $t<\tau$, we have

$$
\begin{gathered}
v^{\varepsilon}(t)=e^{-A_{\varepsilon}^{+}(t-\tau)} \eta+\int_{t}^{\tau} e^{-A_{\varepsilon}^{+}(t-r)} H_{\varepsilon}\left(v^{\varepsilon}(r), s_{*}^{\varepsilon}\left(v^{\varepsilon}(r)\right)\right) d r \\
v^{0}(t)=e^{-A_{0}(t-\tau)} M_{\varepsilon} \eta+\int_{t}^{\tau} e^{-A_{0}(t-r)} f_{0}\left(v^{0}(r)\right) d r
\end{gathered}
$$

then

$$
\begin{aligned}
\left\|v^{\varepsilon}(t)-E_{\varepsilon} v^{0}(t)\right\|_{X_{\varepsilon}^{\alpha}} \leq & \left\|\left(e^{-A_{\varepsilon}^{+}(t-\tau)}-E_{\varepsilon} e^{-A_{0}(t-\tau)} M_{\varepsilon}\right) \eta\right\|_{X_{\varepsilon}^{\alpha}} \\
& +\int_{t}^{\tau}\left\|e^{-A_{\varepsilon}^{+}(t-r)} H_{\varepsilon}\left(v^{\varepsilon}(r), s_{*}^{\varepsilon}\left(v^{\varepsilon}(r)\right)\right)-E_{\varepsilon} e^{-A_{0}(t-r)} f_{0}\left(v^{0}(r)\right)\right\|_{X_{\varepsilon}^{\alpha}} d r .
\end{aligned}
$$

We can write

$$
\begin{aligned}
\int_{t}^{\tau}\left\|e^{-A_{\varepsilon}^{+}(t-r)} H_{\varepsilon}\left(v^{\varepsilon}(r), s_{*}^{\varepsilon}\left(v^{\varepsilon}(r)\right)\right)-E_{\varepsilon} e^{-A_{0}(t-r)} f_{0}\left(v^{0}(r)\right)\right\|_{X_{\varepsilon}^{\alpha}} d r \\
\quad \leq \int_{t}^{\tau}\left\|e^{-A_{\varepsilon}^{+}(t-r)} H_{\varepsilon}\left(v^{\varepsilon}(r), s_{*}^{\varepsilon}\left(v^{\varepsilon}(r)\right)\right)-E_{\varepsilon} e^{-A_{0}(t-r)} M_{\varepsilon} H_{\varepsilon}\left(v^{\varepsilon}(r), s_{*}^{\varepsilon}\left(v^{\varepsilon}(r)\right)\right)\right\|_{X_{\varepsilon}^{\alpha}} d r \\
\quad+\int_{t}^{\tau}\left\|E_{\varepsilon} e^{-A_{0}(t-r)} M_{\varepsilon}\left[H_{\varepsilon}\left(v^{\varepsilon}(r), s_{*}^{\varepsilon}\left(v^{\varepsilon}(r)\right)\right)-H_{\varepsilon}\left(E_{\varepsilon} v^{0}(r), 0\right)\right]\right\|_{X_{\varepsilon}^{\alpha}} d r \\
\quad+\int_{t}^{\tau}\left\|E_{\varepsilon} e^{-A_{0}(t-r)} M_{\varepsilon}\left[H_{\varepsilon}\left(E_{\varepsilon} v^{0}(r), 0\right)-E_{\varepsilon} f_{0}\left(v^{0}(r)\right)\right]\right\|_{X_{\varepsilon}^{\alpha}} d r .
\end{aligned}
$$


If we denote $\varphi(t)=\left\|v^{\varepsilon}(t)-E_{\varepsilon} v^{0}(t)\right\|_{X_{\varepsilon}^{\alpha}} e^{\gamma(t-\tau)}$, by (3.8), (3.14), (3.15) and the Lemmas 3.2.9 and 3.2.10, we obtain

$$
\varphi(t) \leq C(\tau(\varepsilon)+\rho(\varepsilon))+\rho M \gamma^{-1}\left\|s_{*}^{\varepsilon}\right\| \mid+\rho M \int_{t}^{\tau} \varphi(r) d r,
$$

which implies by Gronwall's inequality

$$
\left\|v^{\varepsilon}(t)-E_{\varepsilon} v^{0}(t)\right\|_{X_{\varepsilon}^{\alpha}} \leq\left[C(\tau(\varepsilon)+\rho(\varepsilon))+\rho M \gamma^{-1}\left\|s_{*}^{\varepsilon}\right\| \|\right] e^{(\rho M+\gamma)(\tau-t)},
$$

thus

$$
\left\|s_{*}^{\varepsilon}(\eta)\right\|_{X_{\varepsilon}^{\alpha}} \leq C(\tau(\varepsilon)+\rho(\varepsilon))+\left[\rho M \beta^{-1}+\frac{\rho^{2} M^{2} \gamma^{-1}}{\beta-\gamma-\rho M}\right]\left\|s_{*}^{\varepsilon}\right\| .
$$

It follows from (3.15) that $\left\|s_{*}^{\varepsilon}\right\| \mid \leq C(\tau(\varepsilon)+\rho(\varepsilon))$.

It remains that $\mathscr{M}_{\varepsilon}$ is exponentially attracting and $\mathscr{A}_{\varepsilon} \subset \mathscr{M}_{\varepsilon}$. Let $\left(v^{\varepsilon}, z^{\varepsilon}\right) \in Y_{\varepsilon} \oplus Z_{\varepsilon}$ be the solution of (3.13) and define $\xi^{\varepsilon}(t)=z^{\varepsilon}-s_{*}^{\varepsilon}\left(v^{\varepsilon}(t)\right)$ and consider $y^{\varepsilon}(r, t), r \leq t, t \geq 0$, the solution of

$$
\left\{\begin{array}{l}
y_{t}^{\varepsilon}+A_{\varepsilon}^{+} y^{\varepsilon}=H_{\varepsilon}\left(y^{\varepsilon}, s_{*}^{\varepsilon}\left(y^{\varepsilon}\right)\right), \quad r \leq t \\
y^{\varepsilon}(t, t)=v^{\varepsilon}(t)
\end{array}\right.
$$

Thus,

$$
\begin{aligned}
\left\|y^{\varepsilon}(r, t)-v^{\varepsilon}(r)\right\|_{X_{\varepsilon}^{\alpha}} & \\
= & \left\|\int_{t}^{r} e^{-A_{\varepsilon}^{+}(r-\theta)}\left[H_{\mathcal{\varepsilon}}\left(y^{\varepsilon}(\theta, t), s_{*}^{\varepsilon}\left(y^{\varepsilon}(\theta, t)\right)\right)-H_{\varepsilon}\left(v^{\varepsilon}(\theta), z^{\varepsilon}(\theta)\right)\right] d \theta\right\|_{X_{\varepsilon}^{\alpha}} \\
& \leq \rho M \int_{r}^{t} e^{-\gamma(r-\theta)}\left[(1+\Delta)\left\|y^{\varepsilon}(\theta, t)-v^{\varepsilon}(\theta)\right\|_{X_{\varepsilon}^{\alpha}}+\left\|\xi^{\varepsilon}(\theta)\right\|_{X_{\varepsilon}^{\alpha}}\right] d \theta .
\end{aligned}
$$

By Gronwall's inequality

$$
\left\|y^{\varepsilon}(r, t)-v^{\varepsilon}(r)\right\|_{X_{\varepsilon}^{\alpha}} \leq \rho M \int_{r}^{t} e^{-(-\gamma-\rho M(1+\Delta))(\theta-r)}\left\|\xi^{\varepsilon}(\theta)\right\|_{X_{\varepsilon}^{\alpha}} d \theta \quad r \leq t .
$$

Now we take $t_{0} \in[r, t]$ and then

$$
\begin{aligned}
\| y^{\varepsilon}(r, t) & -y^{\varepsilon}\left(r, t_{0}\right) \|_{X_{\varepsilon}^{\alpha}} \\
& =\left\|e^{-A_{\varepsilon}^{+}\left(r-t_{0}\right)}\left[y\left(t_{0}, t\right)-v^{\varepsilon}\left(t_{0}\right)\right]\right\|_{X_{\varepsilon}^{\alpha}} \\
& +\left\|\int_{t_{0}}^{r} e^{-A_{\varepsilon}^{+}(r-\theta)}\left[H_{\varepsilon}\left(y^{\varepsilon}(\theta, t), s_{*}^{\varepsilon}\left(y^{\varepsilon}(\theta, t)\right)\right)-H_{\varepsilon}\left(y^{\varepsilon}\left(\theta, t_{0}\right), s_{*}^{\varepsilon}\left(y^{\varepsilon}\left(\theta, t_{0}\right)\right)\right)\right] d \theta\right\|_{X_{\varepsilon}^{\alpha}} \\
& \leq \rho M^{2} e^{-\gamma\left(r-t_{0}\right)} \int_{t_{0}}^{t} e^{-(-\gamma-\rho M(1+\Delta))\left(\theta-t_{0}\right)}\left\|\xi^{\varepsilon}(\theta)\right\|_{X_{\varepsilon}^{\alpha}} d \theta \\
& +\rho M \int_{r}^{t_{0}} e^{-\gamma(r-\theta)}(1+\Delta)\left\|y^{\varepsilon}(\theta, t)-y^{\varepsilon}\left(\theta, t_{0}\right)\right\|_{X_{\varepsilon}^{\alpha}} d \theta
\end{aligned}
$$

By Gronwall's inequality

$$
\left\|y^{\varepsilon}(r, t)-y^{\varepsilon}\left(r, t_{0}\right)\right\|_{X_{\varepsilon}^{\alpha}} \leq \rho M^{2} \int_{t_{0}}^{t} e^{-(-\gamma-\rho M(1+\Delta))(\theta-r)}\left\|\xi^{\varepsilon}(\theta)\right\|_{X_{\varepsilon}^{\alpha}} d \theta
$$


Since

$$
z^{\varepsilon}(t)=e^{-A_{\varepsilon}^{-}\left(t-t_{0}\right)} z^{\varepsilon}\left(t_{0}\right)+\int_{t_{0}}^{t} e^{-A_{\varepsilon}^{-}(t-r)} G_{\mathcal{\varepsilon}}\left(v^{\varepsilon}(r), z^{\varepsilon}(r)\right) d r,
$$

we can estimate $\xi^{\varepsilon}(t)$ as

$$
\begin{gathered}
e^{\beta\left(t-t_{0}\right)}\left\|\xi^{\varepsilon}(t)\right\|_{X_{\varepsilon}^{\alpha}} \leq M\left\|\xi^{\varepsilon}\left(t_{0}\right)\right\|_{X_{\varepsilon}^{\alpha}}+\left[\rho M+\frac{\rho^{2} M^{2}(1+\Delta)}{\beta-\gamma-\rho M(1+\Delta)}\right] \int_{t_{0}}^{t} e^{\beta\left(r-t_{0}\right)}\left\|\xi^{\varepsilon}(r)\right\|_{X_{\varepsilon}^{\alpha}} d r \\
+\frac{\rho^{2} M^{3}(1+\Delta)}{\beta-\gamma-\rho M(1+\Delta)} \int_{t_{0}}^{t} e^{-\left(\beta-\gamma-\rho M(1+\Delta)\left(\theta-t_{0}\right)\right.} e^{\beta\left(\theta-t_{0}\right)}\left\|\xi^{\varepsilon}(\theta)\right\|_{X_{\varepsilon}^{\alpha}} d \theta \\
\leq M\left\|\xi^{\varepsilon}\left(t_{0}\right)\right\|_{X_{\varepsilon}^{\alpha}}+\left[\rho M+\frac{\rho^{2} M^{2}(1+\Delta)(1+M)}{\beta-\gamma-\rho M(1+\Delta)}\right] \int_{t_{0}}^{t} e^{\beta\left(r-t_{0}\right)}\left\|\xi^{\varepsilon}(r)\right\|_{X_{\varepsilon}^{\alpha}} d r .
\end{gathered}
$$

By Gronwall's inequality

$$
\left\|\xi^{\varepsilon}(t)\right\|_{X_{\varepsilon}^{\alpha}} \leq M\left\|\xi^{\varepsilon}\left(t_{0}\right)\right\|_{X_{\varepsilon}^{\alpha}} e^{-(L-\beta)\left(t-t_{0}\right)}
$$

and then

$$
\left\|z^{\varepsilon}(t)-s_{*}^{\varepsilon}\left(v^{\varepsilon}(t)\right)\right\|_{X_{\varepsilon}^{\alpha}}=\left\|\xi^{\varepsilon}(t)\right\|_{X_{\varepsilon}^{\alpha}} \leq M\left\|\xi^{\varepsilon}\left(t_{0}\right)\right\|_{X_{\varepsilon}^{\alpha}} e^{-(L-\beta)\left(t-t_{0}\right)} .
$$

Now if $u^{\varepsilon}:=T_{\varepsilon}(t) u_{0}^{\varepsilon}=v^{\varepsilon}(t)+z^{\varepsilon}(t), t \in \mathbb{R}$, denotes the solution through at $u_{0}^{\varepsilon}=v_{0}^{\varepsilon}+z_{0}^{\varepsilon} \in$ $\mathscr{A}_{\varepsilon}$, then

$$
\left\|z^{\varepsilon}(t)-s_{*}^{\varepsilon}\left(v^{\varepsilon}(t)\right)\right\|_{X_{\varepsilon}^{\alpha}} \leq M\left\|z_{0}^{\varepsilon}-s_{*}^{\varepsilon}\left(v_{0}^{\varepsilon}\right)\right\|_{X_{\varepsilon}^{\alpha}} e^{-(L-\beta)\left(t-t_{0}\right)} .
$$

Since $\left\{T_{\varepsilon}(t) u_{0}^{\varepsilon} ; t \in \mathbb{R}\right\} \subset \mathscr{A}_{\varepsilon}$ is bounded, letting $t_{0} \rightarrow-\infty$ we obtain $T_{\varepsilon}(t) u_{0}^{\varepsilon}=v^{\varepsilon}(t)+s_{*}^{\varepsilon}\left(v^{\varepsilon}(t)\right) \in$ $\mathscr{M}_{\varepsilon}$. That is $\mathscr{A}_{\varepsilon} \subset \mathscr{M}_{\varepsilon}$. Moreover, if $B_{\varepsilon} \subset X_{\varepsilon}^{\alpha}$ is a bounded set and $u_{0}^{\varepsilon}=v_{0}^{\varepsilon}+z_{0}^{\varepsilon} \in B_{\varepsilon}$, and we conclude that $T_{\varepsilon}(t) u_{0}^{\varepsilon}=v^{\varepsilon}(t)+z^{\varepsilon}(t)$ satisfies

$$
\begin{aligned}
\sup _{u_{0}^{\varepsilon} \in B_{\varepsilon}} \inf _{w \in \mathscr{M}_{\varepsilon}}\left\|T_{\mathcal{\varepsilon}}(t) u_{0}^{\varepsilon}-w\right\|_{X_{\varepsilon}^{\alpha}} & \leq \sup _{u_{0}^{\varepsilon} \in B_{\varepsilon}}\left\|z^{\varepsilon}(t)-s_{*}^{\varepsilon}\left(v^{\varepsilon}(t)\right)\right\|_{X_{\varepsilon}^{\alpha}} \\
& \leq M e^{-(L-\beta)\left(t-t_{0}\right)} \sup _{u_{0}^{\varepsilon} \in B_{\varepsilon}}\left\|z_{0}^{\varepsilon}-s_{*}^{\varepsilon}\left(v_{0}^{\varepsilon}\right)\right\|_{X_{\varepsilon}^{\alpha}},
\end{aligned}
$$

which implies

$$
\operatorname{dist}_{\varepsilon}\left(T_{\varepsilon}(t) B_{\varepsilon}, \mathscr{M}_{\varepsilon}\right) \leq C e^{-(L-\beta)\left(t-t_{0}\right)},
$$

and thus the proof is complete.

Remark 3.3.2. It is well known (see (SANTAMARIA; ARRIETA, 2014) and (SELL; YOU, 2002)) the $C^{0}, C^{1}$ and $C^{1, \theta}$ convergences of invariant manifolds. That is

$$
\left\|s_{*}^{\varepsilon}\right\|_{C^{0}},\left\|s_{*}^{\varepsilon}\right\|_{C^{1}},\left\|s_{*}^{\varepsilon}\right\|_{C^{1, \theta}} \stackrel{\varepsilon \rightarrow 0}{\longrightarrow} 0 .
$$

\subsection{Rate of Convergence}

In this section we will estimate the convergence of attractors $\mathscr{A}_{\varepsilon}$ of (3.2) to the attractor $\mathscr{A}_{0}$ of the (3.1) in the Hausdorff's metric. The arguments used in this section are inspired by the 
arguments used in (SANTAMARIA; ARRIETA, 2014), where Shadowing Theory was used to estimate the convergence of attractors for semigroups in $\mathbb{R}^{n}$ see Section 2.5 in Chapter 2.

Our main result is that infinite dimensional system which can be regarded as MorseSmale ordinary differential equation, does not have loss when we pass the estimate of resolvent operators to estimate of attractors. The key idea is that we can reduce the systems to the invariant manifold and use the properties of finite dimension.

We saw in the last section that the invariant manifold $\mathscr{M}_{\varepsilon}, \varepsilon \in\left(0, \varepsilon_{0}\right]$, contains the attractor $\mathscr{A}_{\varepsilon}$ and the flow is given by

$$
u^{\varepsilon}(t)=v^{\varepsilon}(t)+s_{*}^{\varepsilon}\left(v^{\varepsilon}(t)\right), \quad t \in \mathbb{R},
$$

where $v^{\varepsilon}(t)$ satisfy the following ordinary differential equation

$$
\dot{v}^{\varepsilon}+A_{\varepsilon}^{+} v^{\varepsilon}=Q_{\varepsilon} f\left(v^{\varepsilon}+s_{*}^{\varepsilon}\left(v^{\varepsilon}(t)\right)\right)
$$

Since $v^{\varepsilon} \in Y_{\varepsilon}$ we can consider $H_{\varepsilon}\left(v^{\varepsilon}\right)=Q_{\varepsilon} f\left(v^{\varepsilon}+s_{*}^{\varepsilon}\left(v^{\varepsilon}(t)\right)\right)$ a continuously differentiable map in $Y_{\varepsilon}$. We denote $\tilde{T}_{\varepsilon}(t)$ the nonlinear semigroup generated by the solution of (3.16), $\tilde{T}_{\varepsilon}=\tilde{T}_{\varepsilon}(1)$ and $T_{0}=T_{0}(1)$.

Now we are ready to estimate the nonlinear semigroup reduced to the invariant manifold.

Theorem 3.4.1. There is a positive constant $C$ independent of $\varepsilon$ such that

$$
\left\|\tilde{T}_{\varepsilon}-E_{\varepsilon} T_{0} M_{\mathcal{\varepsilon}}\right\|_{\mathscr{L}\left(Y_{\varepsilon}, X_{\varepsilon}^{\alpha}\right)} \leq C(\tau(\varepsilon)+\rho(\varepsilon))
$$

and for each $w^{\varepsilon} \in \mathscr{A}_{\varepsilon}$ and $w^{0} \in \mathscr{A}_{0}$,

$$
\left\|E_{\varepsilon} T_{0} M_{\varepsilon} Q_{\varepsilon} w^{\varepsilon}-E_{\varepsilon} T_{0} w^{0}\right\|_{X_{\varepsilon}^{\alpha}} \leq C\left\|Q_{\varepsilon} w^{\varepsilon}-E_{\varepsilon} w^{0}\right\|_{X_{\varepsilon}^{\alpha}} .
$$

Proof. For $\bar{w}^{\varepsilon} \in Y_{\varepsilon}$ we have

$$
\begin{aligned}
\left\|\tilde{T}_{\mathcal{\varepsilon}}(t) \bar{w}^{\varepsilon}-E_{\varepsilon} T_{0}(t) M_{\mathcal{\varepsilon}} \bar{w}^{\varepsilon}\right\|_{X_{\varepsilon}^{\alpha}} \leq\left\|\left(e^{-A_{\varepsilon}^{+} t}-E_{\varepsilon} e^{-A_{0} t} M_{\mathcal{\varepsilon}}\right) \bar{w}^{\varepsilon}\right\|_{X_{\varepsilon}^{\alpha}} \\
+\int_{0}^{t}\left\|e^{-A_{\varepsilon}^{+}(t-s)} H_{\mathcal{\varepsilon}}\left(\tilde{T}_{\mathcal{\varepsilon}}(s) \bar{w}^{\varepsilon}\right)-E_{\varepsilon} e^{-A_{0}(t-s)} f_{0}\left(T_{0}(s) M_{\mathcal{E}} \bar{w}^{\varepsilon}\right)\right\|_{X_{\varepsilon}^{\alpha}} d s .
\end{aligned}
$$

But

$$
\begin{aligned}
\int_{0}^{t} \| e^{-A_{\varepsilon}^{+}(t-s)} & H_{\varepsilon}\left(\tilde{T}_{\mathcal{\varepsilon}}(s) \bar{w}^{\varepsilon}\right)-E_{\varepsilon} e^{-A_{0}(t-s)} f_{0}\left(T_{0}(s) M_{\mathcal{\varepsilon}} \bar{w}^{\varepsilon}\right) \|_{X_{\varepsilon}^{\alpha}} d s \\
& \leq \int_{0}^{t}\left\|e^{-A_{\varepsilon}^{+}(t-s)} H_{\mathcal{\varepsilon}}\left(\tilde{T}_{\mathcal{\varepsilon}}(s) \bar{w}^{\varepsilon}\right)-E_{\varepsilon} e^{-A_{0}(t-s)} M_{\mathcal{\varepsilon}} H_{\mathcal{\varepsilon}}\left(\tilde{T}_{\mathcal{\varepsilon}}(s) \bar{w}^{\varepsilon}\right)\right\|_{X_{\varepsilon}^{\alpha}} d s \\
& +\int_{0}^{t}\left\|E_{\varepsilon} e^{-A_{0}(t-s)} M_{\varepsilon}\left[H_{\varepsilon}\left(\tilde{T}_{\varepsilon}(s) \bar{w}^{\varepsilon}\right)-H_{\varepsilon}\left(E_{\varepsilon} T_{0}(s) M_{\varepsilon} \bar{w}^{\varepsilon}\right)\right]\right\|_{X_{\varepsilon}^{\alpha}} d s \\
& +\int_{0}^{t}\left\|E_{\varepsilon} e^{-A_{0}(t-s)}\left[M_{\varepsilon} H_{\varepsilon}\left(E_{\varepsilon} T_{0}(s) M_{\mathcal{E}} \bar{w}^{\varepsilon}\right)-E_{\varepsilon} f_{0}\left(T_{0}(s) M_{\mathcal{\varepsilon}} \bar{w}^{\varepsilon}\right)\right]\right\|_{X_{\varepsilon}^{\alpha}} d s
\end{aligned}
$$


If we denote the three last integrals by $I_{1}, I_{2}$ and $I_{3}$ respectively and consider $0 \leq t \leq 1$, we have from (3.8) and the Lemmas 3.2.9 and 3.2.10 that

and

$$
I_{1} \leq C \tau(\varepsilon), \quad I_{2} \leq C \int_{0}^{t}\left\|\tilde{T}_{\varepsilon}(s) \bar{w}^{\varepsilon}-E_{\varepsilon} T_{0}(s) M_{\varepsilon} \bar{w}^{\varepsilon}\right\|_{X_{\varepsilon}^{\alpha}} d s
$$

$$
\begin{aligned}
I_{3} & \leq C \int_{0}^{t}\left\|M_{\varepsilon} H_{\varepsilon}\left(E_{\varepsilon} T_{0}(s) M_{\varepsilon} \bar{w}^{\varepsilon}\right)-f_{0}\left(T_{0}(s) M_{\varepsilon} \bar{w}^{\varepsilon}\right)\right\|_{X_{0}} d s \\
& \leq C \int_{0}^{t}\left\|H_{\varepsilon}\left(E_{\varepsilon} T_{0}(s) M_{\varepsilon} \bar{w}^{\varepsilon}\right)-E_{\varepsilon} f_{0}\left(T_{0}(s) M_{\varepsilon} \bar{w}^{\varepsilon}\right)\right\|_{X_{\varepsilon}^{\alpha}} d s \\
& \leq C \int_{0}^{t}\left\|Q_{\varepsilon} f_{\varepsilon}\left(E_{\varepsilon} T_{0}(s) M_{\varepsilon} \bar{w}^{\varepsilon}+s_{*}^{\varepsilon}\left(E_{\varepsilon} T_{0}(s) M_{\varepsilon} \bar{w}^{\varepsilon}\right)\right)-E_{\varepsilon} f_{0}\left(T_{0}(s) M_{\varepsilon} \bar{w}^{\varepsilon}\right)\right\|_{X_{\varepsilon}^{\alpha}} d s \\
& \leq C \int_{0}^{t}\left\|Q_{\varepsilon} f_{\varepsilon}\left(E_{\varepsilon} T_{0}(s) M_{\varepsilon} \bar{w}^{\varepsilon}+s_{*}^{\varepsilon}\left(E_{\varepsilon} T_{0}(s) M_{\varepsilon} \bar{w}^{\varepsilon}\right)\right)-Q_{\varepsilon} E_{\varepsilon} f_{0}\left(T_{0}(s) M_{\varepsilon} \bar{w}^{\varepsilon}\right)\right\|_{X_{\varepsilon}^{\alpha}} d s \\
& +C \int_{0}^{t}\left\|Q_{\varepsilon} E_{\varepsilon} f_{0}\left(T_{0}(s) M_{\varepsilon} \bar{w}^{\varepsilon}\right)-E_{\varepsilon} f_{0}\left(T_{0}(s) M_{\varepsilon} \bar{w}^{\varepsilon}\right)\right\|_{X_{\varepsilon}^{\alpha}} d s \\
& \leq C\left(\left\|s_{*}^{\varepsilon}\right\|+\rho(\varepsilon)\right)+C \tau(\varepsilon) .
\end{aligned}
$$

Hence

$$
\left\|\tilde{T}_{\mathcal{\varepsilon}}(t) \bar{w}^{\varepsilon}-E_{\varepsilon} T_{0}(t) M_{\mathcal{\varepsilon}} \bar{w}^{\varepsilon}\right\|_{X_{\varepsilon}^{\alpha}} \leq C(\tau(\varepsilon)+\rho(\varepsilon))+C \int_{0}^{t}\left\|\tilde{T}_{\varepsilon}(s) \bar{w}^{\varepsilon}-E_{\varepsilon} T_{0}(s) M_{\varepsilon} \bar{w}^{\varepsilon}\right\|_{X_{\varepsilon}^{\alpha}} d s .
$$

By Gronwall's inequality and then taking $t=1$ we obtain (3.17).

Now, to prove (3.18), note that from variation of constants formula and the Gronwall's inequality, we have $T_{0}(\cdot)$ a Lipschitz map in $X_{0}$, thus

$$
\begin{aligned}
\left\|E_{\varepsilon} T_{0} M_{\varepsilon} Q_{\varepsilon} w^{\varepsilon}-E_{\varepsilon} T_{0} w^{0}\right\|_{X_{\varepsilon}^{\alpha}} & \leq C\left\|T_{0} M_{\varepsilon} Q_{\varepsilon} w^{\varepsilon}-T_{0} w^{0}\right\|_{X_{0}} \\
& \leq C\left\|M_{\varepsilon} Q_{\varepsilon} w^{\varepsilon}-w^{0}\right\|_{X_{0}} \\
& =C\left\|M_{\varepsilon} Q_{\varepsilon} w^{\varepsilon}-M_{\varepsilon} E_{\varepsilon} w^{0}\right\|_{X_{0}} \\
& \leq C\left\|Q_{\varepsilon} w^{\varepsilon}-E_{\varepsilon} w^{0}\right\|_{X_{\varepsilon}^{\alpha}} .
\end{aligned}
$$

As a consequence of Theorem 3.4.1 we have the following result.

Theorem 3.4.2. Let $\mathscr{A}_{\varepsilon}$ be the attractor for (3.2) and $\mathscr{A}_{0}$ the attractor of the (3.1). Then there is a positive constant $C$ independent of $\varepsilon$ such that

$$
\mathrm{d}_{\varepsilon}\left(\mathscr{A}_{\varepsilon}, E_{\varepsilon} \mathscr{A}_{0}\right) \leq C(\tau(\varepsilon)+\rho(\varepsilon))+\mathrm{d}_{\varepsilon}\left(Q_{\varepsilon} \mathscr{A}_{\varepsilon}, E_{\varepsilon} \mathscr{A}_{0}\right) .
$$

Proof. Let $u^{\varepsilon} \in \mathscr{A}_{\varepsilon}$, and $u^{0} \in \mathscr{A}_{0}$. We can write $u^{0}=T_{0} z^{0}, z^{0} \in \mathscr{A}_{0}$ and $u^{\varepsilon}=v^{\varepsilon}+s_{*}^{\varepsilon}\left(v^{\varepsilon}\right)$, with $v^{\varepsilon}=\tilde{T}_{\varepsilon} z^{\varepsilon}, z^{\varepsilon} \in Q_{\varepsilon} \mathscr{A}_{\varepsilon}$. Thus, by (3.17) and (3.18), we have

$$
\begin{aligned}
\left\|u^{\varepsilon}-E_{\varepsilon} u^{0}\right\|_{X_{\varepsilon}^{\alpha}} & =\left\|v^{\varepsilon}+s_{*}^{\varepsilon}\left(v^{\varepsilon}\right)-E_{\varepsilon} T_{0} z^{0}\right\|_{X_{\varepsilon}^{\alpha}} \\
& \leq\left\|\tilde{T}_{\varepsilon} z^{\varepsilon}+s_{*}^{\varepsilon}\left(\tilde{T}_{\varepsilon} z^{\varepsilon}\right)-E_{\varepsilon} T_{0} z^{0}\right\|_{X_{\varepsilon}^{\alpha}} \\
& \leq\left\|\tilde{T}_{\varepsilon} z^{\varepsilon}-E_{\varepsilon} T_{0} M_{\varepsilon} z^{\varepsilon}\right\|_{X_{\varepsilon}^{\alpha}}+\left\|s_{*}^{\varepsilon}\right\|+\left\|E_{\varepsilon} T_{0} M_{\varepsilon} z^{\varepsilon}-E_{\varepsilon} T_{0} z^{0}\right\|_{X_{\varepsilon}^{\alpha}} \\
& \leq C(\tau(\varepsilon)+\rho(\varepsilon))+C\left\|z^{\varepsilon}-E_{\varepsilon} z^{0}\right\|_{X_{\varepsilon}^{\alpha}} .
\end{aligned}
$$


Recall that we denoted $\left\{\varphi_{i}^{0}\right\}_{i=1}^{n}$ the eigenfunctions associated with the $n$ eigenvalues of $A_{0}$. Since $Y_{\varepsilon}$ and $X_{0}$ are $n$ dimensional, we can consider

$$
Y_{\varepsilon}=\operatorname{span}\left[Q_{\varepsilon} E_{\varepsilon} \varphi_{1}^{0}, \cdots, Q_{\varepsilon} E_{\varepsilon} \varphi_{n}^{0}\right] \quad \text { and } \quad X_{0}=\operatorname{span}\left[\varphi_{1}^{0}, \cdots, \varphi_{n}^{0}\right]
$$

and define the isomorphisms $j_{\varepsilon}: Y_{\varepsilon} \rightarrow \mathbb{R}^{n}$ and $j_{0}: X_{0} \rightarrow \mathbb{R}^{n}$ by

$$
\sum_{j=1}^{n} z_{j}^{\varepsilon} Q_{\varepsilon} E_{\varepsilon} \varphi_{j}^{0} \stackrel{j_{\varepsilon}}{\longrightarrow}\left(z_{1}^{\varepsilon}, \cdots, z_{n}^{\varepsilon}\right) \quad \text { and } \quad \sum_{j=1}^{n} z_{j}^{0} \varphi_{j}^{0} \stackrel{j_{0}}{\longrightarrow}\left(z_{1}^{0}, \cdots, z_{n}^{0}\right),
$$

where in $\mathbb{R}^{n}$ we consider the following norm

$$
\|z\|_{\mathbb{R}^{n}}=\left(\sum_{j=1}^{n} z_{i}^{2}\left(\lambda_{i}^{\varepsilon}\right)^{2 \alpha}\right)^{\frac{1}{2}}, \quad z=\left(z_{1}, \cdots, z_{n}\right) \in \mathbb{R}^{n},
$$

where $\left\{\lambda_{i}^{\varepsilon}\right\}_{j=1}^{n}$ is the first $n$ eigenvalues of $A_{\varepsilon}$.

Lemma 3.4.3. For $\bar{w}^{\varepsilon} \in Y_{\varepsilon}$ and $w^{0} \in X_{0}$ we have the following inequality

$$
\left\|j_{\varepsilon} \bar{w}^{\varepsilon}-j_{0} w^{0}\right\|_{\mathbb{R}^{n}} \leq C\left(\left\|\bar{w}^{\varepsilon}-E_{\varepsilon} w^{0}\right\|_{X_{\varepsilon}^{\alpha}}+\tau(\varepsilon)\right),
$$

where $C$ is a constant independent of $\varepsilon$.

Proof. We have,

$$
\begin{aligned}
\bar{w}^{\varepsilon}-E_{\varepsilon} w^{0} & =\sum_{j=1}^{n} z_{j}^{\varepsilon} Q_{\varepsilon} E_{\varepsilon} \varphi_{j}^{0}-E_{\varepsilon} \sum_{j=1}^{n} z_{j}^{0} \varphi_{j}^{0} \\
& =\left(Q_{\varepsilon} E_{\varepsilon}-E_{\varepsilon}\right) \sum_{j=1}^{n} z_{j}^{\varepsilon} \varphi_{j}^{0}+E_{\varepsilon} \sum_{j=1}^{n}\left(z_{j}^{\varepsilon}-z_{j}^{0}\right) \varphi_{j}^{0},
\end{aligned}
$$

since $M_{\varepsilon} E_{\varepsilon}=I_{X_{0}}$, we obtain

$$
\sum_{j=1}^{n}\left(z_{j}^{\varepsilon}-z_{j}^{0}\right) \varphi_{j}^{0}=M_{\mathcal{\varepsilon}}\left(\bar{w}^{\varepsilon}-E_{\varepsilon} w^{0}\right)-M_{\mathcal{\varepsilon}}\left(Q_{\varepsilon} E_{\varepsilon}-E_{\varepsilon}\right) \sum_{j=1}^{n} z_{j}^{\varepsilon} \varphi_{j}^{0},
$$

which implies

$$
\left\|j_{\varepsilon} \bar{w}^{\varepsilon}-j_{0} w^{0}\right\|_{\mathbb{R}^{n}} \leq C\left(\left\|\bar{w}^{\varepsilon}-E_{\varepsilon} w^{0}\right\|_{X_{\varepsilon}^{\alpha}}+\left\|Q_{\varepsilon} E_{\varepsilon}-E_{\varepsilon}\right\|_{\mathscr{L}\left(X_{0}, X_{\varepsilon}^{\alpha}\right)}\right)
$$

The result follows by Lemma 3.11.

Now we restrict our attention to dynamical systems $\tilde{T}_{\varepsilon}$ and $T_{0}$ that acts in $Y_{\varepsilon}$ and $X_{0}$ respectively. Thus we consider the systems generated by following ordinary differential equations,

$$
\left\{\begin{array}{l}
\dot{v}^{\varepsilon}(t)+A_{\varepsilon}^{+} v^{\varepsilon}(t)=H_{\varepsilon}\left(v^{\varepsilon}(t)\right), \quad t \in \mathbb{R}, \\
v^{\varepsilon}(0)=\bar{w}^{\varepsilon} \in Y_{\varepsilon}, \quad \varepsilon \in\left(0, \varepsilon_{0}\right],
\end{array}\right.
$$




$$
\left\{\begin{array}{l}
\dot{u}^{0}(t)+A_{0} u^{0}(t)=f_{0}\left(u^{0}(t)\right), \quad t \in \mathbb{R} \\
u^{0}(0)=M_{\varepsilon} \bar{w}^{\varepsilon} \in X_{0}
\end{array}\right.
$$

Let $z_{0}^{0}, z_{0}^{\varepsilon} \in \mathbb{R}^{n}$ such that $\bar{w}^{\varepsilon}=j_{\mathcal{\varepsilon}}^{-1} z_{0}^{\varepsilon}$ and $M_{\varepsilon} \bar{w}^{\varepsilon}=j_{0}^{-1} z_{0}^{0}$, then $j_{\varepsilon}\left(v^{\varepsilon}(t)\right)$ and $j_{0}\left(u^{0}(t)\right)$ satisfy the following equation in $\mathbb{R}^{n}$,

$$
\begin{aligned}
& \left\{\begin{array}{l}
\dot{z}^{\varepsilon}(t)+j_{\varepsilon} A_{\varepsilon}^{+} j_{\varepsilon}^{-1} z^{\varepsilon}(t)=j_{\varepsilon} H_{\varepsilon}\left(j_{\varepsilon}^{-1} z^{\varepsilon}(t)\right), \quad t \in \mathbb{R}, \\
z^{\varepsilon}(0)=z_{0}^{\varepsilon}, \quad \varepsilon \in\left(0, \varepsilon_{0}\right],
\end{array}\right. \\
& \left\{\begin{array}{l}
\dot{z}^{0}(t)+j_{0} A_{0} j_{0}^{-1} z^{0}(t)=j_{0} f_{0}\left(j_{0}^{-1} z^{0}(t)\right), \quad t \in \mathbb{R}, \\
z^{0}(0)=z_{0}^{0}
\end{array}\right.
\end{aligned}
$$

Since we assume that the limiting problem (3.1) generates a Morse-Smale semigroup in $X_{0}$, the perturbed problem (3.2) generate a Morse-Smale semigroup in $X_{\varepsilon}^{\alpha}$, thus if we denote $S_{0}$ and $S_{\varepsilon}$ the time one map of the systems (3.19) and (3.20), we have $S_{0}$ and $S_{\varepsilon}$ Morse-Smale semigroups and if we denote $\overline{\mathscr{A}}_{0}$ and $\overline{\mathscr{A}}_{\varepsilon}$ are its attractors respectively, by Theorem 2.5.10, we have

$$
\operatorname{dist}_{\mathbb{R}^{n}}\left(\overline{\mathscr{A}}_{\mathcal{\varepsilon}}, \overline{\mathscr{A}}_{0}\right) \leq C\left\|S_{\varepsilon}-S_{0}\right\|_{L^{\infty}\left(\mathbb{R}^{n}, \mathbb{R}^{n}\right)}
$$

where $C$ is a constant independent of $\varepsilon$.

Next we estimate the convergence of the nonlinear semigroups acting in $\mathbb{R}^{n}$.

Proposition 3.4.4. There is a constant $C$ independent of $\varepsilon$ such that

$$
\left\|S_{\varepsilon}-S_{0}\right\|_{L^{\infty}\left(\mathbb{R}^{n}, \mathbb{R}^{n}\right)} \leq C(\tau(\varepsilon)+\rho(\varepsilon))
$$

Proof. We have

$$
\left\|S_{\varepsilon}-S_{0}\right\|_{L^{\infty}\left(\mathbb{R}^{n}, \mathbb{R}^{n}\right)}=\sup _{z \in \mathbb{R}^{n}}\left\|z^{\varepsilon}(1)-z^{0}(1)\right\|_{\mathbb{R}^{n}}=\sup _{z \in \mathbb{R}^{n}}\left\|j_{\varepsilon}\left(v^{\varepsilon}(1)\right)-j_{0}\left(u^{0}(1)\right)\right\|_{\mathbb{R}^{n}}
$$

where $z^{0}(0)=z=z^{\varepsilon}(0)$, that is $j_{0}^{-1}(z)=u^{0}(0)=M_{\varepsilon} \bar{w}^{\varepsilon}$ and $j_{\varepsilon}^{-1}(z)=v^{\varepsilon}(0)=\bar{w}^{\varepsilon}$. By the Lemma 3.4.3, we have

$$
\begin{aligned}
\left\|j_{\varepsilon}\left(v^{\varepsilon}(1)\right)-j_{0}\left(u^{0}(1)\right)\right\|_{\mathbb{R}^{n}} & \leq C\left(\left\|v^{\varepsilon}(1)-E_{\varepsilon} u^{0}(1)\right\|_{X_{\varepsilon}^{\alpha}}+\tau(\varepsilon)\right) \\
& =C\left(\left\|\tilde{T}_{\varepsilon} w^{\varepsilon}-E_{\varepsilon} T_{0} M_{\varepsilon} \bar{w}^{\varepsilon}\right\|_{X_{\varepsilon}^{\alpha}}+\tau(\varepsilon)\right) .
\end{aligned}
$$

The result follows by Theorem 3.4.1.

The next result relates the projected attractors with the attractors in $\mathbb{R}^{n}$.

Theorem 3.4.5. There is a constant $C$ independent of $\varepsilon$ such that

$$
\mathrm{d}_{\varepsilon}\left(Q_{\varepsilon} \mathscr{A}_{\varepsilon}, E_{\varepsilon} \mathscr{A}_{0}\right) \leq C\left(\operatorname{dist}_{\mathbb{R}^{n}}\left(\overline{\mathscr{A}}_{\varepsilon}, \overline{\mathscr{A}}_{0}\right)+\tau(\varepsilon)\right) .
$$


Proof. Let $w^{0} \in \mathscr{A}_{0}$ and $w^{\varepsilon} \in \mathscr{A}_{\varepsilon}$, we can write

$$
w^{0}=j_{0}^{-1}\left(z^{0}\right)=\sum_{j=1}^{n} z_{j}^{0} \varphi_{j}^{0}, \quad \text { and } \quad Q_{\varepsilon} w^{\varepsilon}=j_{\varepsilon}^{-1}\left(z^{\varepsilon}\right)=\sum_{j=1}^{n} z_{j}^{\varepsilon} \psi_{j}^{\varepsilon},
$$

where $\psi_{j}^{\varepsilon}=Q_{\varepsilon} E_{\varepsilon} \varphi_{j}^{0}, j=1, \ldots, n, z^{0} \in \overline{\mathscr{A}}_{0}$ and $z^{\varepsilon} \in \overline{\mathscr{A}}_{\varepsilon}$. Thus

$$
\begin{aligned}
\left\|Q_{\varepsilon} w^{\varepsilon}-E_{\varepsilon} w^{0}\right\|_{X_{\varepsilon}^{\alpha}} & =\left\|j_{\varepsilon}^{-1}\left(z^{\varepsilon}\right)-E_{\varepsilon} j_{0}^{-1}\left(z^{0}\right)\right\|_{X_{\varepsilon}^{\alpha}}=\left\|\sum_{j=1}^{n} z_{j}^{\varepsilon} \psi_{j}^{\varepsilon}-E_{\varepsilon} \sum_{j=1}^{n} z_{j}^{0} \varphi_{j}^{0}\right\|_{X_{\varepsilon}^{\alpha}} \\
& \leq\left\|\sum_{j=1}^{n} z_{j}^{\varepsilon}\left(\psi_{j}^{\varepsilon}-E_{\varepsilon} \varphi_{j}^{0}\right)\right\|_{X_{\varepsilon}^{\alpha}}+\left\|\sum_{j=1}^{n}\left(z_{j}^{\varepsilon}-z_{j}^{0}\right) E_{\varepsilon} \varphi_{j}^{0}\right\|_{X_{\varepsilon}^{\alpha}}
\end{aligned}
$$

But

$$
\begin{aligned}
\left\|\sum_{j=1}^{n} z_{j}^{\varepsilon}\left(\psi_{j}^{\varepsilon}-E_{\varepsilon} \varphi_{j}^{0}\right)\right\|_{X_{\varepsilon}^{\alpha}}= & \left\|\sum_{j=1}^{n} z_{j}^{\varepsilon}\left(Q_{\varepsilon} E_{\varepsilon} \varphi_{j}^{0}-E_{\varepsilon} \varphi_{j}^{0}\right)\right\|_{X_{\varepsilon}^{\alpha}} \\
& \leq \sup _{1 \leq j \leq n}\left|z_{j}^{\varepsilon}\right|\left\|\left(Q_{\varepsilon} E_{\varepsilon}-E_{\varepsilon}\right) \sum_{j=1}^{n} \varphi_{j}^{0}\right\|_{X_{\varepsilon}^{\alpha}} \\
& \leq C\left\|Q_{\varepsilon} E_{\varepsilon}-E_{\varepsilon}\right\|_{\mathscr{L}\left(X_{0}, X_{\varepsilon}^{\alpha}\right)}
\end{aligned}
$$

and

$$
\begin{aligned}
\left\|\sum_{j=1}^{n}\left(z_{j}^{\varepsilon}-z_{j}^{0}\right) E_{\varepsilon} \varphi_{j}^{0}\right\|_{X_{\varepsilon}^{\alpha}}^{2} & =\sum_{j=1}^{n}\left(\lambda_{j}^{\varepsilon}\right)^{2 \alpha}\left\|Q_{j}^{\varepsilon} \sum_{i=1}^{n}\left(z_{i}^{\varepsilon}-z_{i}^{0}\right) E_{\varepsilon} \varphi_{i}^{0}\right\|_{X_{\varepsilon}}^{2} \\
& \leq \sum_{j=1}^{n}\left(\lambda_{j}^{\varepsilon}\right)^{2 \alpha}\left(z_{j}^{\varepsilon}-z_{j}^{0}\right)^{2}\left\|Q_{j}^{\varepsilon} E_{\varepsilon} \varphi_{j}^{0}\right\|_{X_{\varepsilon}}^{2} \\
& \leq C \sum_{j=1}^{n}\left(\lambda_{j}^{\varepsilon}\right)^{2 \alpha}\left(z_{j}^{\varepsilon}-z_{j}^{0}\right)^{2} \\
& =C\left\|z^{\varepsilon}-z^{0}\right\|_{\mathbb{R}^{n}}^{2} .
\end{aligned}
$$

The result now follows by Lemma 3.2.9.

Now we are ready to prove the main result of this chapter.

Theorem 3.4.6. Let $\mathscr{A}_{\varepsilon}$ be the attractor for (3.2) and $\mathscr{A}_{0}$ the attractor of the (3.1). Then there is a positive constant $C$ independent of $\varepsilon$ such that

$$
\mathrm{d}_{\varepsilon}\left(\mathscr{A}_{\varepsilon}, E_{\varepsilon} \mathscr{A}_{0}\right) \leq C(\tau(\varepsilon)+\rho(\varepsilon))
$$

Proof. The proof follows from estimate (3.21), Proposition 3.4.4 and Theorem 3.4.5.

\subsection{Further Comments}

In this section we consider a more general class of linear operators than that seen in the Section 3.1. Here the operators have spectrum set more general than operator with compact 
resolvent. We will observe that for this class of operators, that was considered in (CARVALHO et al., 2010), the theory developed in the previous section can be applied with some additional a priori estimates on the linear semigroups.

Definition 3.5.1. We say that a family of linear operators $\left\{A_{\varepsilon}\right\}_{\varepsilon \in\left(0, \varepsilon_{0}\right]}$ is in the class $G\left(X_{\varepsilon}, C, \omega\right)$ if each operator $A_{\varepsilon}: D\left(A_{\varepsilon}\right) \subset X_{\varepsilon} \rightarrow X_{\varepsilon}$ generates a strongly continuous semigroup of linear operator $\left\{e^{-A_{\varepsilon} t}: t \geq 0\right\} \subset \mathscr{L}\left(X_{\varepsilon}\right)$ and

$$
\left\|e^{-A_{\varepsilon} t}\right\|_{\mathscr{L}\left(X_{\varepsilon}, X_{\varepsilon}^{\alpha}\right)} \leq C e^{\omega t}, \quad \forall t \geq 0
$$

for some constant $C \geq 1$ and $\omega \in \mathbb{R}$ independent of $\varepsilon$.

Definition 3.5.2. We say that $\left\{A_{\varepsilon}\right\}_{\varepsilon \in\left(0, \varepsilon_{0}\right]}$ is of class $H\left(X_{\varepsilon}, M, \theta\right)$ if $\rho\left(-A_{\varepsilon}\right)$ contains the same sector

$$
\Sigma_{\theta}=\left\{\lambda \in \mathbb{C}:|\arg (\lambda)| \leq \frac{\pi}{2}+\theta\right\}
$$

and moreover

$$
\left\|\left(\lambda+A_{\varepsilon}\right)^{-1}\right\|_{\mathscr{L}\left(X_{\varepsilon}, X_{\varepsilon}^{\alpha}\right)} \leq \frac{M}{|\lambda|+1}, \quad \forall \lambda \in \Sigma_{\theta}, \varepsilon \in\left(0, \varepsilon_{0}\right]
$$

where $\theta \in(0, \pi / 2)$ and $M \geq 0$ do not dependent on $\varepsilon$.

We have $H\left(X_{\varepsilon}, M, \theta\right) \subset G\left(X_{\mathcal{\varepsilon}}, C, \omega\right)$. If we consider the problems (3.1) and (3.2) with the family $\left\{A_{\varepsilon}\right\}_{\varepsilon \in\left(0, \varepsilon_{0}\right]} \in H\left(X_{\mathcal{\varepsilon}}, M, \theta\right)$ and if we assume that we can obtain the linear estimates in the Lemma 3.2.10, then the Theorems 3.3.1 and 3.4.6 also are valid in this context. 


\section{PARABOLIC EQUATIONS WITH LARGE DIFFUSION}

Many models of chemical, biological and ecological systems are described by reactiondiffusion equations with large diffusion coefficients. These kind of problems display solutions that show no spatial dependence in large time behavior. In these cases the flow is essentially determined by a system of ordinary differential equation, see (CONLEY; HOFF; SMOLLER, 1978) and (HALE, 1986) for results concerning solutions to homogeneous Neumann boundary conditions, i.e., when there is no flux across the boundary. When for large time the solutions are almost independent of the space variable it is said to occur spatial homogenization. This is the case when the diffusion is large in all domain, in fact, it is well known that large diffusivity implies a fast redistribution of the spatial inhomogeneities, therefore the solutions approaches, as time increases, a constant function given by its average in phase space. If the physical domain of the equation is bounded we can take the average of the solution in this domain in order to get the limiting ordinary differential equation that will describe the asymptotic behavior. Indeed a singular limiting problem is obtained and in this context we can consider small singular perturbation in the sense that all equilibrium points and connections between them are preserved. Hence spatial homogenization is of great interest to identify the dynamic of infinite dimensional systems that seems more complicated than finite dimensional dynamical system.

\subsection{Spatial Homogenization I}

Using the approach developed in the Chapter 3 we consider a reaction diffusion equation with large diffusion in all parts of the domain. We start doing a analysis of the large diffusion effects in order to obtain a limiting ordinary differential equation. Here we consider the convergences in one appropriated energy space and we show that the attractors of perturbed problems are closed to the attractor for the limiting problem. Our main reference is the paper 
(RODRíGUEZ-BERNAL; WILLIE, 2005).

We consider the parabolic problem

$$
\left\{\begin{array}{l}
u_{t}^{\varepsilon}-\operatorname{div}\left(p_{\varepsilon}(x) \nabla u^{\varepsilon}\right)+\left(\lambda+V_{\varepsilon}(x)\right) u^{\varepsilon}=f\left(u^{\varepsilon}\right), \quad x \in \Omega, t>0 \\
\frac{\partial u^{\varepsilon}}{\partial \vec{n}}=0, \quad x \in \partial \Omega \\
u^{\varepsilon}(0)=u_{0}^{\varepsilon}
\end{array}\right.
$$

where $0<\varepsilon \leq \varepsilon_{0}, \Omega \subset \mathbb{R}^{n}$ is a bounded smooth open connected set, $\partial \Omega$ is the boundary of $\Omega$ and $\frac{\partial u^{\varepsilon}}{\partial \vec{n}}$ is the co-normal derivative operator with $\vec{n}$ the unit outward normal vector to $\partial \Omega$. We assume the potentials $V_{\varepsilon} \in L^{p}(\Omega)$ with

$$
p \begin{cases}\geq 1, & n=1 \\ \geq 2, & n \geq 2\end{cases}
$$

and $V_{\varepsilon}$ converges to a constant $V_{0} \in \mathbb{R}$ in $L^{p}(\Omega)$, that is, we consider $\tau(\varepsilon)$ an increasing positive function of $\varepsilon$, with $\tau(0)=0$, such that

$$
\left\|V_{\varepsilon}-V_{0}\right\|_{L^{p}(\Omega)} \leq \tau(\varepsilon) \stackrel{\varepsilon \rightarrow 0}{\longrightarrow} 0
$$

Note that (4.2) implies that the spatial average of $V_{\varepsilon}$ converges to $V_{0}$ as $\varepsilon \rightarrow 0$, that is

$$
\frac{1}{|\Omega|} \int_{\Omega} V_{\varepsilon} \stackrel{\varepsilon \rightarrow 0}{\longrightarrow} V_{0}
$$

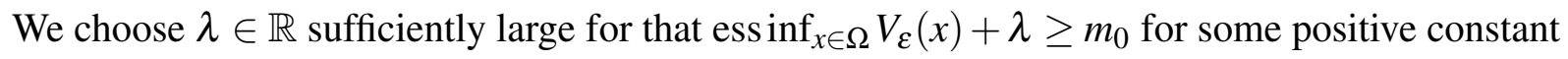
$m_{0}$. Moreover we will assume the diffusion is large in $\Omega$, that is, for each $\varepsilon \in\left(0, \varepsilon_{0}\right]$ the map $p_{\varepsilon}$ is positive smooth defined in $\bar{\Omega}$ satisfying

$$
p(\varepsilon):=\min _{x \in \bar{\Omega}}\left\{p_{\varepsilon}(x)\right\} \stackrel{\varepsilon \rightarrow 0}{\longrightarrow} \infty \quad \text { with } \quad 0<m_{0} \leq p_{\varepsilon}(x), \quad \forall x \in \Omega
$$

Since large diffusivity implies fast homogenization, we expect, for small values of $\varepsilon$, that the solution of this problem converge to a function spatially constant in $\Omega$. Indeed, we have

$$
\int_{\Omega} u_{t}^{\varepsilon}(t, x) d x-\int_{\partial \Omega} \operatorname{div}\left(p_{\varepsilon}(x) \nabla u^{\varepsilon}(t, x)\right) d x+\int_{\Omega}\left(\lambda+V_{\varepsilon}(x)\right) u^{\varepsilon}(t, x) d x=\int_{\Omega} f\left(u^{\varepsilon}(t, x)\right) d x,
$$

it follows from Divergence Theorem that

$$
\int_{\Omega} u_{t}^{\varepsilon}(t, x) d x+\int_{\partial \Omega} p_{\varepsilon}(x) \frac{\partial u^{\varepsilon}}{\partial \vec{n}} d x+\int_{\Omega}\left(\lambda+V_{\varepsilon}(x)\right) u^{\varepsilon}(t, x) d x=\int_{\Omega} f\left(u^{\varepsilon}(t, x)\right) d x .
$$

Taking the average on $\Omega$, the limiting problem as $\varepsilon$ goes to zero is given by ordinary differential equation

$$
\left\{\begin{array}{l}
\dot{u}^{0}+\left(\lambda+V_{0}\right) u^{0}=f\left(u^{0}\right), \quad t>0 \\
u^{0}(0)=u_{0}^{0}
\end{array}\right.
$$


which (RODRíGUEZ-BERNAL; WILLIE, 2005) proves to determine the asymptotic behavior of (4.1).

In this chapter we are concerning in how fast the dynamics of the problem (4.1) approaches the dynamics of the problem (4.3). Following the results on the previous chapter we estimate this convergence by functions $\tau(\varepsilon)$ and $p(\varepsilon)$.

Since we have established the limit problem we need to study the well posedness of (4.1) and (4.3) as abstract parabolic equation in appropriated Banach spaces. To this end, we define the operator $A_{\varepsilon}: \mathscr{D}\left(A_{\varepsilon}\right) \subset L^{2}(\Omega) \rightarrow L^{2}(\Omega)$ by

$$
\mathscr{D}\left(A_{\varepsilon}\right)=\left\{u \in H^{2}(\Omega): \frac{\partial u^{\varepsilon}}{\partial \vec{n}}=0\right\}, \quad A_{\varepsilon} u=-\operatorname{div}\left(p_{\varepsilon} \nabla u\right)+\left(\lambda+V_{\varepsilon}\right) u .
$$

We denote $L_{\Omega}^{2}=\left\{u \in H^{1}(\Omega): \nabla u=0\right.$ in $\left.\Omega\right\}$ and we define the operator $A_{0}: L_{\Omega}^{2} \subset L^{2}(\Omega) \rightarrow$ $L^{2}(\Omega)$ by $A_{0} u=\left(\lambda+V_{0}\right) u$. Note that $L_{\Omega}^{2}$ is the set of all almost everywhere constant function in $\Omega$.

It is well known that $A_{\varepsilon}$ is a positive invertible operator with compact resolvent for each $\varepsilon \in\left[0, \varepsilon_{0}\right]$ see Section 2.2 in Chapter 2. Hence we define in the usual way (see(HENRY, 1980)), the fractional power space $X_{\varepsilon}^{\frac{1}{2}}=H^{1}(\Omega), \varepsilon \in\left(0, \varepsilon_{0}\right]$, and $X_{0}^{\frac{1}{2}}=L_{\Omega}^{2}$ with the scalar products

$$
\begin{gathered}
\langle u, v\rangle_{X_{\varepsilon}^{\frac{1}{2}}}=\int_{\Omega} p_{\varepsilon} \nabla u \nabla v d x+\int_{\Omega}\left(\lambda+V_{\varepsilon}\right) u v d x, \quad u, v \in X_{\varepsilon}^{\frac{1}{2}}, \varepsilon \in\left(0, \varepsilon_{0}\right] ; \\
\langle u, v\rangle_{X_{0}^{\frac{1}{2}}}=|\Omega|^{-1}\left(\lambda+V_{0}\right) u v, \quad u, v \in X_{0}^{\frac{1}{2}} .
\end{gathered}
$$

The space $X_{0}^{\frac{1}{2}}$ is a one dimensional closed subspace of $X_{\mathcal{\varepsilon}}^{\frac{1}{2}}, \varepsilon \in\left(0, \varepsilon_{0}\right]$ and $X_{\mathcal{\varepsilon}}^{\frac{1}{2}} \subset H^{1}(\Omega)$ with injection constant independent of $\varepsilon$, but the injection $H^{1}(\Omega) \subset X_{\varepsilon}^{\frac{1}{2}}$ is not uniform, in fact is valid

$$
m_{0}\|u\|_{H^{1}}^{2} \leq\|u\|_{X_{\mathcal{\varepsilon}}^{\frac{1}{2}}}^{2} \leq M(\varepsilon)\|u\|_{H^{1}}^{2}
$$

with $M(\varepsilon) \rightarrow \infty$ as $\varepsilon \rightarrow 0$ and we will show in the Corollary 4.1.2 that there is no positive constant $C$ independent of $\varepsilon$ such that $\|u\|_{X_{\varepsilon}^{\frac{1}{2}}}^{2} \leq C\|u\|_{H^{1}}^{2}$. Therefore bounds for solutions in the Sobolev spaces does not give suitable estimates in the fractional power space, even though we consider $X_{\mathcal{\varepsilon}}^{\frac{1}{2}}$ as phase space.

If we denote the Nemitskii functional of $f$ by the same notation $f$, then (4.1) and (4.3) can be written as

$$
\left\{\begin{array}{l}
u_{t}^{\varepsilon}+A_{\varepsilon} u^{\varepsilon}=f\left(u^{\varepsilon}\right), \\
u^{\varepsilon}(0)=u_{0}^{\varepsilon} \in X_{\varepsilon}^{\frac{1}{2}}, \quad \varepsilon \in\left[0, \varepsilon_{0}\right]
\end{array}\right.
$$

We assume $f$ is continuously differentiable and the equilibrium set of (4.4) for $\varepsilon=0$ is composed of a finite number of hyperbolic equilibrium points. That is

$$
\mathscr{E}_{0}:=\left\{u \in D\left(A_{0}\right): A_{0} u-f(u)=0\right\}=\left\{x_{*}^{1,0}<x_{*}^{2,0} \leq \ldots \leq x_{*}^{m, 0}\right\}
$$


and $\sigma\left(A_{0}-f^{\prime}\left(u_{*}^{i, 0}\right)\right) \cap\{\mu: \operatorname{Re}(\mu)=0\}=\emptyset$, for $i \in\{1, \ldots, m\}$.

In order to ensure that all solution of (4.4) are globally defined, and there is a global attractor for the nonlinear semigroup given by theses solutions, we assume the following conditions.

(i) If $n=2$, for all $\eta>0$, there is a constant $C_{\eta}>0$ such that

$$
|f(u)-f(v)| \leq C_{\eta}\left(e^{\eta|u|^{2}}+e^{\eta|v|^{2}}\right)|u-v|, \quad \forall u, v \in \mathbb{R},
$$

and if $n \geq 3$, there is a constant $\tilde{C}>0$ such that

$$
|f(u)-f(v)| \leq \tilde{C}|u-v|\left(|u|^{\frac{4}{n-2}}+|v|^{\frac{4}{n-2}}+1\right), \quad \forall u, v \in \mathbb{R} .
$$

(ii)

$$
\limsup _{|u| \rightarrow \infty} \frac{f(u)}{u}<0
$$

Under theses assumptions (ARRIETA; CARVALHO; RODRíGUEZ-BERNAL, 1999; ARRIETA; CARVALHO; RODRíGUEZ-BERNAL, 2000a) and (HALE, 1988) ensure that the problem (4.4) is globally well posed and generate a nonlinear semigroup satisfying

$$
T_{\varepsilon}(t) u_{0}^{\varepsilon}=e^{-A t} u_{0}^{\varepsilon}+\int_{0}^{t} e^{-A(t-s)} f\left(T_{\varepsilon}(s) u_{0}^{\varepsilon}\right) d s, \quad t \geq 0 .
$$

Moreover there is a global attractor $\mathscr{A}_{\mathcal{E}}$ for $T_{\mathcal{E}}(\cdot)$ uniformly bounded in $X_{\mathcal{\varepsilon}}^{\frac{1}{2}}$, that is

$$
\sup _{\varepsilon \in\left[0, \varepsilon_{0}\right]} \sup _{w \in \mathscr{A} \varepsilon}\|w\|_{X_{\varepsilon}^{\frac{1}{2}}}<\infty .
$$

We also have $T_{0}(\cdot)$ is a Morse-Smale semigroup and $\mathscr{A}_{0}=\left[x_{*}^{1,0}, x_{*}^{m, 0}\right]$.

In order to find a rate of convergence for the resolvent operators we consider the projection

$$
P u=\frac{1}{|\Omega|} \int_{\Omega} u d x, \quad u \in L^{2}(\Omega) \quad \text { or } \quad u \in X_{\varepsilon}^{\frac{1}{2}}
$$

Thus $P$ is an orthogonal projection acting on $L^{2}$ onto $L_{\Omega}^{2}$ or $X_{\mathcal{\varepsilon}}^{\frac{1}{2}}$ onto $X_{0}^{\frac{1}{2}}$.

Lemma 4.1.1. For $g \in L^{2}(\Omega)$ with $\|g\|_{L^{2}} \leq 1$ and $\varepsilon \in\left(0, \varepsilon_{0}\right]$, let $u^{\varepsilon}$ be the solution of elliptic problem

$$
\left\{\begin{array}{l}
-\operatorname{div}\left(p_{\varepsilon}(x) \nabla u^{\varepsilon}\right)+\left(\lambda+V_{\varepsilon}(x)\right) u^{\varepsilon}=g, \quad x \in \Omega \\
\frac{\partial u^{\varepsilon}}{\partial \vec{n}}=0, \quad x \in \partial \Omega
\end{array}\right.
$$

Then there is a constant $C>0$, independent of $\varepsilon$, such that

$$
\left\|u^{\varepsilon}-u^{0}\right\|_{X_{\varepsilon}^{\frac{1}{2}}} \leq C\left(\tau(\varepsilon)+p(\varepsilon)^{-\frac{1}{2}}\right)
$$

where $u^{0}=\frac{P g}{\lambda+V_{0}}$. 
Proof. The weak solution $u^{\varepsilon}$ satisfies

$$
\begin{gathered}
\int_{\Omega} p_{\varepsilon} \nabla u^{\varepsilon} \nabla \varphi d x+\int_{\Omega}\left(\lambda+V_{\varepsilon}\right) u^{\varepsilon} \varphi d x=\int_{\Omega} g \varphi d x, \quad \forall \varphi \in X_{\varepsilon}^{\frac{1}{2}}, \varepsilon \in\left(0, \varepsilon_{0}\right] ; \\
\int_{\Omega}\left(\lambda+V_{0}\right) u^{0} \varphi d x=\int_{\Omega} \operatorname{Pg} \varphi d x, \quad \forall \varphi \in X_{0}^{\frac{1}{2}} .
\end{gathered}
$$

With the aid of the projection $P$ we have

$$
\begin{gathered}
\int_{\Omega} p_{\varepsilon}\left|\nabla u^{\varepsilon}\right|^{2} d x+\int_{\Omega}\left(\lambda+V_{\varepsilon}\right) u^{\varepsilon}\left(u^{\varepsilon}-u^{0}\right) d x=\int_{\Omega} g\left(u^{\varepsilon}-u^{0}\right) d x \\
\int_{\Omega}\left(\lambda+V_{0}\right) u^{0}\left(P u^{\varepsilon}-u^{0}\right) d x=\int_{\Omega} P g\left(P u^{\varepsilon}-u^{0}\right) d x
\end{gathered}
$$

which implies

$$
\int_{\Omega} g\left(u^{\varepsilon}-u^{0}\right) d x-\int_{\Omega} P g\left(P u^{\varepsilon}-u^{0}\right) d x=\int_{\Omega} g(I-P) u^{\varepsilon} d x
$$

and

$$
\begin{gathered}
\int_{\Omega} p_{\varepsilon}\left|\nabla u^{\varepsilon}\right|^{2} d x+\int_{\Omega}\left(\lambda+V_{\varepsilon}\right) u^{\varepsilon}\left(u^{\varepsilon}-u^{0}\right) d x-\int_{\Omega}\left(\lambda+V_{0}\right) u^{0}\left(P u^{\varepsilon}-u^{0}\right) d x \\
=\left\|u^{\varepsilon}-u^{0}\right\|_{X_{\varepsilon}^{\frac{1}{2}}}^{2}+\int_{\Omega}\left(V_{\varepsilon}-V_{0}\right) u^{0}\left(u^{\varepsilon}-u^{0}\right) d x .
\end{gathered}
$$

Therefore

$$
\left\|u^{\varepsilon}-u^{0}\right\|_{X_{\varepsilon}^{\frac{1}{2}}}^{2} \leq \int_{\Omega}\left|V_{\varepsilon}-V_{0} \| u^{0}\right|\left|u^{\varepsilon}-u^{0}\right| d x+\int_{\Omega}\left|g(I-P) u^{\varepsilon}\right| d x .
$$

If $n=1$, we have $X_{\varepsilon}^{\frac{1}{2}} \subset H^{1} \subset L^{\infty}$, thus

$$
\int_{\Omega}\left|V_{\varepsilon}-V_{0}\left\|u^{0}\right\| u^{\varepsilon}-u^{0}\right| d x \leq C\left\|u^{\varepsilon}-u^{0}\right\|_{L^{\infty}}\left\|V^{\varepsilon}-V_{0}\right\|_{L^{1}} \leq C\left\|u^{\varepsilon}-u^{0}\right\|_{X_{\varepsilon}^{\frac{1}{2}}} \tau(\varepsilon) .
$$

If $n \geq 2$, we have $L^{p} \subset L^{2}$, thus

$$
\int_{\Omega}\left|V_{\varepsilon}-V_{0}\right|\left|u^{0}\left\|u^{\varepsilon}-u^{0} \mid d x \leq C\right\| u^{\varepsilon}-u^{0}\left\|_{L^{2}}\right\| V^{\varepsilon}-V_{0}\left\|_{L^{2}} \leq C\right\| u^{\varepsilon}-u^{0} \|_{X_{\varepsilon}^{\frac{1}{2}}} \tau(\varepsilon) .\right.
$$

By Poincaré's inequality for average, we have

$$
\int_{\Omega}\left|g(I-P) u^{\varepsilon}\right| d x \leq\|g\|_{L^{2}}\left(\int_{\Omega}\left|\nabla u^{\varepsilon}\right|^{2} d x\right)^{\frac{1}{2}}
$$

but

$$
p(\varepsilon) \int_{\Omega}\left|\nabla u^{\varepsilon}\right|^{2} d x \leq \int_{\Omega} p_{\varepsilon}\left|\nabla u^{\varepsilon}-\nabla u^{0}\right|^{2} d x \leq\left\|u^{\varepsilon}-u^{0}\right\|_{X_{\varepsilon}^{\frac{1}{2}}}^{2}
$$

Putting these estimates together the result follows.

With the aid of the Lemma 4.1.1 we can prove that the constant of immersion of $H^{1}(\Omega) \subset X_{\varepsilon}^{\frac{1}{2}}$ is not uniform in $\varepsilon$. 
Corollary 4.1.2. There is no positive constant $C$ independent of $\varepsilon$ such that

$$
\|u\|_{X_{\varepsilon}^{\frac{1}{2}}}^{2} \leq C\|u\|_{H^{1}}^{2} \quad \forall u \in X_{\varepsilon}^{\frac{1}{2}}
$$

Proof. If there is such a constant $C$ take $v^{\varepsilon}=(I-P) u^{\varepsilon}$ as given by the Lemma 4.1.1, thus by Poincaré's inequality for average, we have

$$
\begin{aligned}
p(\varepsilon)\left\|v^{\varepsilon}\right\|_{H^{1}}^{2} & \leq p(\varepsilon) \int_{\Omega}\left|\nabla v^{\varepsilon}\right|^{2} d x+\int_{\Omega}\left|v^{\varepsilon}\right|^{2} d x \\
& \leq C p(\varepsilon) \int_{\Omega}\left|\nabla v^{\varepsilon}\right|^{2} d x \\
& \leq C\left\|v^{\varepsilon}\right\|_{X_{\varepsilon}^{\frac{1}{2}}}^{2} \leq C\left\|v^{\varepsilon}\right\|_{H^{1}}^{2} .
\end{aligned}
$$

The convergence of the resolvent operators can be stated as follows.

Corollary 4.1.3. There is a positive constant $C$ independent of $\varepsilon$ such that

$$
\left\|A_{\varepsilon}^{-1}-A_{0}^{-1} P\right\|_{\mathscr{L}\left(L^{2}, X_{\varepsilon}^{\frac{1}{2}}\right)} \leq C\left(\tau(\varepsilon)+p(\varepsilon)^{-\frac{1}{2}}\right) .
$$

Proof. Let $g \in L^{2}(\Omega)$ such that $\|g\|_{L^{2}} \leq 1$ and define $u^{\varepsilon}=A_{\mathcal{\varepsilon}}^{-1} g$ for $\varepsilon \in\left(0, \varepsilon_{0}\right]$ and $u^{0}=P g /(\lambda+$ $\left.V_{0}\right)$. Now the result follows from Lemma 4.1.1.

We are now in position to state the main result in this section.

Theorem 4.1.4. There is an one dimensional invariant manifold $\mathscr{M}_{\varepsilon}$ for (4.4) such that $\mathscr{A}_{\varepsilon} \subset \mathscr{M}_{\varepsilon}$ and the flow on $\mathscr{A}_{\varepsilon}$ can be reduced to an ordinary differential equation. Moreover the convergence of attractors of (4.5) can be estimated by

$$
\mathrm{d}_{\varepsilon}\left(\mathscr{A}_{\varepsilon}, \mathscr{A}_{0}\right) \leq C\left(\tau(\varepsilon)+p(\varepsilon)^{-\frac{1}{2}}\right) .
$$

Proof. If we define $X_{0}=\mathbb{R}=X_{0}^{\frac{1}{2}}, X_{\varepsilon}^{\alpha}=X_{\varepsilon}^{\frac{1}{2}}$ and $X_{\varepsilon}=L^{2}(\Omega), \varepsilon \in\left(0, \varepsilon_{0}\right]$, we have $\left\{A_{\varepsilon}\right\}_{\varepsilon \in\left(0, \varepsilon_{0}\right]}$ a family of operators satisfying the assumptions of the Chapter 3 , where $A_{0}$ has spectrum set given by $\sigma\left(A_{0}\right)=\left\{\lambda+V_{0}\right\}, \lambda+V_{0}>0$ and $A_{\varepsilon}$ has spectrum set given by $\sigma\left(A_{\varepsilon}\right)=\left\{\lambda_{1}^{\varepsilon}, \lambda_{2}^{\varepsilon}, \ldots\right\}$, $\lambda_{1}^{\varepsilon} \geq m_{0}>0$. Note that we have $\operatorname{dim}\left(X_{0}\right)=1$ which implies the limiting ODE (4.3) is one dimensional. Finally we define $E_{\varepsilon}$ by the inclusion $X_{0}^{\frac{1}{2}} \subset X_{\varepsilon}^{\frac{1}{2}}$ and $M_{\varepsilon}=P$, where $P$ is defined in (4.6). Hence the assumptions of the Theorem 3.4.6 are satisfied. The proof is completed.

Due to the simplicity of the dynamics of the Problem (4.1) we can prove the Theorem 4.1.4 without using the Theorem 2.5.10, that is, without Shadowing Theory. In fact, we explore the geometry offer by the finite dimension, see Figure 9. This geometric argument that motivated us to investigate the rate of convergence of attractors for problems which the asymptotic behavior can be described by a system of ordinary differential equation.

First we estimate the convergence of equilibrium points. 
Theorem 4.1.5. Let $u_{*}^{0} \in \mathscr{E}_{0}$. Then for $\varepsilon$ sufficiently small (we still denote $\varepsilon \in\left(0, \varepsilon_{0}\right]$ ), there is $\delta>0$ such that the equation $A_{\varepsilon} u-f(u)=0$ has the only solution $u_{*}^{\varepsilon} \in\left\{u \in X_{\varepsilon}^{\frac{1}{2}} ;\left\|u-u_{*}^{0}\right\|_{X_{\varepsilon}^{\frac{1}{2}}} \leq \delta\right\}$. Moreover

$$
\left\|u_{*}^{\varepsilon}-u_{*}^{0}\right\|_{X_{\varepsilon}^{\frac{1}{2}}} \leq C\left(\tau(\varepsilon)+p(\varepsilon)^{-\frac{1}{2}}\right)
$$

Proof. The proof is the same as given in (ARRIETA; BEZERRA; CARVALHO, 2013) and (CARBONE; CARVALHO; SCHIABEL-SILVA, 2008). Here we just need to proof the estimates (4.7). We have $u_{*}^{\varepsilon}$ and $u_{*}^{0}$ given by

$$
u_{*}^{0}=\left(A_{0}+V_{0}\right)^{-1}\left[f\left(u_{*}^{0}\right)+V_{0} u_{*}^{0}\right] \quad \text { and } \quad u_{*}^{\varepsilon}=\left(A_{\varepsilon}+V_{0}\right)^{-1}\left[f\left(u_{*}^{\varepsilon}\right)+V_{0} u_{*}^{\varepsilon}\right]
$$

where $V_{0}=-f^{\prime}\left(u_{*}^{0}\right)$. Thus

$$
\begin{aligned}
\left\|u_{*}^{\varepsilon}-u_{*}^{0}\right\|_{X_{\varepsilon}^{\frac{1}{2}}} & \leq\left\|\left(A_{\varepsilon}+V_{0}\right)^{-1}\left[f\left(u_{*}^{\varepsilon}\right)+V_{0} u_{*}^{\varepsilon}\right]-\left(A_{0}+V_{0}\right)^{-1}\left[f\left(u_{*}^{0}\right)+V_{0} u_{*}^{0}\right]\right\|_{X_{\varepsilon}^{\frac{1}{2}}} \\
& \leq\left\|\left[\left(A_{\varepsilon}+V_{0}\right)^{-1}-\left(A_{0}+V_{0}\right)^{-1} P\right]\left[f\left(u_{*}^{\varepsilon}\right)+V_{0} u_{*}^{\varepsilon}\right]\right\|_{X_{\varepsilon}^{\frac{1}{2}}} \\
& +\left\|\left(A_{0}+V_{0}\right)^{-1} P\left[f\left(u_{*}^{\varepsilon}\right)-f\left(u_{*}^{0}\right)+V_{0}\left(u_{*}^{\varepsilon}-u_{*}^{0}\right)\right]\right\|_{X_{\varepsilon}^{\frac{1}{2}}}
\end{aligned}
$$

We have the following equality

$$
\left(A_{\varepsilon}+V_{0}\right)^{-1}-\left(A_{0}+V_{0}\right)^{-1} P=\left[I-\left(A_{\varepsilon}+V_{0}\right)^{-1} V_{0}\right]\left(A_{\varepsilon}^{-1}-A_{0}^{-1} P\right)\left[I-V_{0}\left(A_{0}+V_{0}\right)^{-1}\right] .
$$

And then $\left\|\left[\left(A_{\varepsilon}+V_{0}\right)^{-1}-\left(A_{0}+V_{0}\right)^{-1} P\right]\left[f\left(u_{*}^{\varepsilon}\right)+V_{0} u_{*}^{\varepsilon}\right]\right\|_{X_{\varepsilon}^{\frac{1}{2}}} \leq C\left(\tau(\varepsilon)+p(\varepsilon)^{-\frac{1}{2}}\right)$.

If we denote $z^{\varepsilon}=f\left(u_{*}^{\varepsilon}\right)-f\left(u_{*}^{0}\right)+V_{0}\left(u_{*}^{\varepsilon}-u_{*}^{0}\right)$, since $f$ is continuously differentiable, for all $\delta>0$ there is $\varepsilon$ sufficiently small such that $\left\|z^{\varepsilon}\right\|_{X_{\varepsilon}^{\frac{1}{2}}} \leq \delta\left\|u_{*}^{\varepsilon}-u_{*}^{0}\right\|_{X_{\varepsilon}^{\frac{1}{2}}}$. Thus

$$
\left\|\left(A_{0}+V_{0}\right)^{-1} P z^{\varepsilon}\right\|_{X_{\varepsilon}^{\frac{1}{2}}} \leq \delta\left\|\left(A_{0}+V_{0}\right)^{-1} P\right\|_{\mathscr{L}\left(L^{2}, X_{\varepsilon}^{\frac{1}{2}}\right)}\left\|u_{*}^{\varepsilon}-u_{*}^{0}\right\|_{X_{\varepsilon}^{\frac{1}{2}}} \cdot
$$

We choice $\delta$ sufficiently small such that $\delta\left\|\left(A_{0}+V_{0}\right)^{-1} P\right\|_{\mathscr{L}\left(L^{2}, X_{\varepsilon}^{\frac{1}{2}}\right)} \leq \frac{1}{2}$, and then

$$
\left\|u_{*}^{\varepsilon}-u_{*}^{0}\right\|_{X_{\varepsilon}^{\frac{1}{2}}} \leq C\left(\tau(\varepsilon)+p(\varepsilon)^{-\frac{1}{2}}\right)+\frac{1}{2}\left\|u_{*}^{\varepsilon}-u_{*}^{0}\right\|_{X_{\varepsilon}^{\frac{1}{2}}} .
$$

We now give a geometric prove of the Theorem 4.1.4.

Proof. By triangle inequality, we have

$$
\begin{aligned}
\mathrm{d}_{\varepsilon}\left(\mathscr{A}_{\varepsilon}, \mathscr{A}_{0}\right) & \leq \mathrm{d}_{\varepsilon}\left(\mathscr{A}_{\varepsilon}, Q_{\varepsilon} \mathscr{A}_{\varepsilon}\right)+\mathrm{d}_{\varepsilon}\left(Q_{\varepsilon} \mathscr{A}_{\varepsilon}, \mathscr{A}_{0}\right) \\
& \leq \mathrm{d}_{\varepsilon}\left(\mathscr{A}_{\varepsilon}, Q_{\varepsilon} \mathscr{A}_{\varepsilon}\right)+\mathrm{d}_{\varepsilon}\left(Q_{\varepsilon} \mathscr{A}_{\varepsilon}, P Q_{\varepsilon} \mathscr{A}_{\varepsilon}\right)+\mathrm{d}_{\varepsilon}\left(P Q_{\varepsilon} \mathscr{A}_{\varepsilon}, \mathscr{A}_{0}\right)
\end{aligned}
$$

We estimate each part. 
Let $z \in \mathscr{A}_{\varepsilon}$, since $\mathscr{A}_{\varepsilon} \subset \mathscr{M}_{\varepsilon}, z=z^{\varepsilon}+s_{*}^{\varepsilon}\left(z^{\varepsilon}\right)$ for some $z^{\varepsilon} \in Q_{\varepsilon} \mathscr{A}_{\varepsilon}$, then

$$
\begin{aligned}
\operatorname{dist}\left(z, Q_{\varepsilon} \mathscr{A}_{\varepsilon}\right) & =\inf _{x \in Q_{\varepsilon}(\bar{\lambda}) \mathscr{A} \mathcal{\varepsilon}}\|z-x\|_{X_{\varepsilon}^{\frac{1}{2}}} \leq\left\|z^{\varepsilon}+s_{*}^{\varepsilon}\left(z^{\varepsilon}\right)-z^{\varepsilon}\right\|_{X_{\varepsilon}^{\frac{1}{2}}}=\left\|s_{*}^{\varepsilon}\left(z^{\varepsilon}\right)\right\|_{X_{\varepsilon}^{\frac{1}{2}}} \\
& \leq\left\|s_{*}^{\varepsilon}\right\| \| C\left(\tau(\varepsilon)+p(\varepsilon)^{-\frac{1}{2}}\right), \\
\operatorname{dist}\left(z^{\varepsilon}, \mathscr{A}_{\varepsilon}\right) & =\inf _{x \in \mathscr{A}_{\varepsilon}}\left\|z^{\varepsilon}-x\right\|_{X_{\varepsilon}^{\frac{1}{2}}} \leq\left\|z^{\varepsilon}-\left(z^{\varepsilon}+s_{*}^{\varepsilon}\left(z^{\varepsilon}\right)\right)\right\|_{X_{\varepsilon}^{\frac{1}{2}}}=\left\|s_{*}^{\varepsilon}\left(z^{\varepsilon}\right)\right\|_{X_{\varepsilon}^{\frac{1}{2}}} \\
& \leq\left\|s_{*}^{\varepsilon}\right\| \leq C\left(\tau(\varepsilon)+p(\varepsilon)^{-\frac{1}{2}}\right),
\end{aligned}
$$

which implies

$$
\mathrm{d}_{\varepsilon}\left(\mathscr{A}_{\varepsilon}, Q_{\varepsilon} \mathscr{A}_{\varepsilon}\right)=\max \left\{\operatorname{dist}_{\varepsilon}\left(\mathscr{A}_{\varepsilon}, Q_{\varepsilon} \mathscr{A}_{\varepsilon}\right), \operatorname{dist}_{\varepsilon}\left(Q_{\varepsilon} \mathscr{A}_{\varepsilon}, \mathscr{A}_{\varepsilon}\right)\right\} \leq C\left(\tau(\varepsilon)+p(\varepsilon)^{-\frac{1}{2}}\right) .
$$

Let $z^{\varepsilon} \in Q_{\varepsilon} \mathscr{A}_{\varepsilon}$ then $z^{\varepsilon}=Q_{\varepsilon} w^{\varepsilon}$ for some $w^{\varepsilon} \in \mathscr{A}_{\varepsilon}$. Since $\bigcup_{\varepsilon \in\left[0, \varepsilon_{0}\right]} \mathscr{A}_{\varepsilon}$ is uniformly bounded in $X_{\varepsilon}^{\frac{1}{2}}$ we can assume $\left\|w^{\varepsilon}\right\|_{X_{\varepsilon}^{\frac{1}{2}}} \leq C$. Thus

$$
\begin{aligned}
\operatorname{dist}\left(z^{\varepsilon}, P Q_{\varepsilon} \mathscr{A}_{\varepsilon}\right) & =\inf _{x \in P Q_{\varepsilon} \mathscr{A}_{\varepsilon}}\left\|z^{\varepsilon}-x\right\|_{X_{\varepsilon}^{\frac{1}{2}}} \leq\left\|z^{\varepsilon}-P z^{\varepsilon}\right\|_{X_{\varepsilon}^{\frac{1}{2}}}=\left\|Q_{\varepsilon} w^{\varepsilon}-P Q_{\varepsilon} w^{\varepsilon}\right\|_{X_{\varepsilon}^{\frac{1}{2}}} \\
& =\left\|\left(Q_{\varepsilon}-P\right) Q_{\varepsilon} w^{\varepsilon}\right\|_{X_{\varepsilon}^{\frac{1}{2}}} \leq C\left(\tau(\varepsilon)+p(\varepsilon)^{-\frac{1}{2}}\right), \\
\operatorname{dist}\left(P z^{\varepsilon}, Q_{\varepsilon} \mathscr{A}_{\varepsilon}\right) & =\inf _{x \in Q_{\varepsilon} \mathscr{A}_{\varepsilon}}\left\|P z^{\varepsilon}-x\right\|_{X_{\varepsilon}^{\frac{1}{2}}} \leq\left\|P z^{\varepsilon}-z^{\varepsilon}\right\|_{X_{\varepsilon}^{\frac{1}{2}}}=\left\|P Q_{\varepsilon} w^{\varepsilon}-Q_{\varepsilon} w^{\varepsilon}\right\|_{X_{\varepsilon}^{\frac{1}{2}}} \\
& =\left\|\left(P-Q_{\varepsilon}\right) Q_{\varepsilon} w^{\varepsilon}\right\|_{X_{\varepsilon}^{\frac{1}{2}}} \leq C\left(\tau(\varepsilon)+p(\varepsilon)^{-\frac{1}{2}}\right),
\end{aligned}
$$

which implies

$$
\begin{aligned}
\mathrm{d}_{\varepsilon}\left(Q_{\varepsilon} \mathscr{A}_{\varepsilon}, P Q_{\varepsilon} \mathscr{A}_{\varepsilon}\right) & =\max \left\{\operatorname{dist}_{\varepsilon}\left(Q_{\varepsilon} \mathscr{A}_{\varepsilon}, P Q_{\varepsilon} \mathscr{A}_{\varepsilon}\right), \operatorname{dist}_{\varepsilon}\left(P Q_{\varepsilon} \mathscr{A}_{\varepsilon}, Q_{\varepsilon} \mathscr{A}_{\varepsilon}\right)\right\} \\
& \leq C\left(\tau(\varepsilon)+p(\varepsilon)^{-\frac{1}{2}}\right) .
\end{aligned}
$$

Finally we have $\mathscr{A}_{0}=\left[x_{*}^{1,0}, x_{*}^{m, 0}\right]$ and $T_{0}(\cdot)$ is a Morse-Smale semigroup. Since MorseSmale semigroup are stable (see (BORTOLAN et al., 2015)) we can assume $\mathscr{A}_{\varepsilon}=\left[x_{*}^{1, \varepsilon}, x_{*}^{m, \varepsilon}\right]$ which implies $Q_{\varepsilon} \mathscr{A}_{\varepsilon}=\left[Q_{\varepsilon} x_{*}^{1, \varepsilon}, Q_{\varepsilon} x_{*}^{m, \varepsilon}\right]$ and $P Q_{\varepsilon} \mathscr{A}_{\varepsilon}=\left[P Q_{\varepsilon} x_{*}^{1, \varepsilon}, P Q_{\varepsilon} x_{*}^{m, \varepsilon}\right]$. Without loss of generality we can assume $P Q_{\varepsilon} x_{*}^{1, \varepsilon} \leq x_{*}^{1,0}$ and $P Q_{\varepsilon} x_{*}^{m, \varepsilon} \leq x_{*}^{m, 0}$. Thus

$$
\mathrm{d}_{\varepsilon}\left(P Q_{\varepsilon} \mathscr{A}_{\varepsilon}, \mathscr{A}_{0}\right)=\left\|P Q_{\varepsilon} x_{*}^{1, \varepsilon}-x_{*}^{1,0}\right\|_{X_{\varepsilon}^{\frac{1}{2}}}+\left\|P Q_{\varepsilon} x_{*}^{m, \varepsilon}-x_{*}^{m, 0}\right\|_{X_{\varepsilon}^{\frac{1}{2}}} \cdot
$$

But

$$
\begin{aligned}
\left\|P Q_{\varepsilon} x_{*}^{1, \varepsilon}-x_{*}^{1,0}\right\|_{X_{\varepsilon}^{\frac{1}{2}}} & =\left\|P Q_{\varepsilon} x_{*}^{1, \varepsilon}-P x_{*}^{1,0}\right\|_{X_{\varepsilon}^{\frac{1}{2}}} \leq\|P\|_{\mathscr{L}\left(L^{2}\right)}\left\|Q_{\varepsilon} x_{*}^{1, \varepsilon}-P x_{*}^{1,0}\right\|_{X_{\varepsilon}^{\frac{1}{2}}} \\
& \leq\|P\|_{\mathscr{L}\left(L^{2}\right)}\left\|Q_{\varepsilon} x_{*}^{1, \varepsilon}-P x_{*}^{1, \varepsilon}\right\|_{X_{\varepsilon}^{\frac{1}{2}}}+\|P\|_{\mathscr{L}\left(L^{2}\right)}\left\|P x_{*}^{1, \varepsilon}-P x_{*}^{1,0}\right\|_{X_{\varepsilon}^{\frac{1}{2}}} \\
& \leq C\left(\tau(\varepsilon)+p(\varepsilon)^{-\frac{1}{2}}\right) .
\end{aligned}
$$

In the same way $\left\|P Q_{\varepsilon} x_{*}^{m, \varepsilon}-x_{*}^{m, 0}\right\|_{X_{\varepsilon}^{\frac{1}{2}}} \leq C\left(\tau(\varepsilon)+p(\varepsilon)^{-\frac{1}{2}}\right)$. 


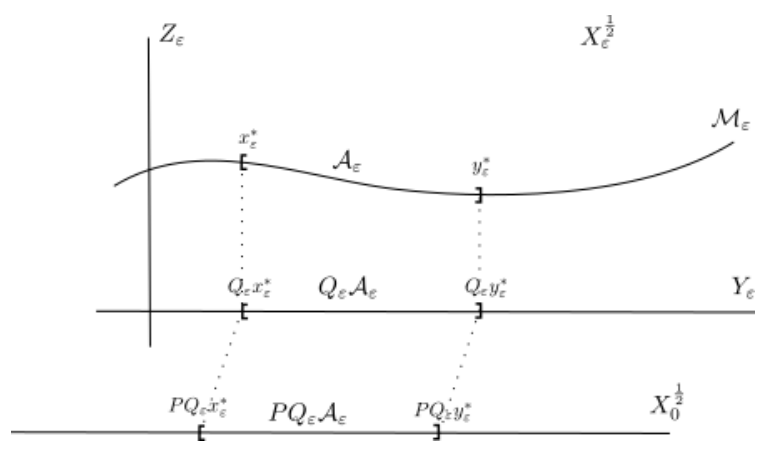

Figure 9 - Geometry of the Phase Space

\subsection{Spatial Homogenization II}

Here we consider a parabolic equation with large diffusion in all domain which the limiting problem is an ordinary differential equation in $\mathbb{R}^{N}$. Again we use the theory developed in Chapter 3 to estimate the convergence of attractors. Our main reference is (CARVALHO et al., 2010).

Let $\Omega$ be a bounded open set in $\mathbb{R}^{N}$ with $\Gamma=\partial \Omega$ smooth. Consider the system of reaction-diffusion equations of the form

$$
\left\{\begin{array}{l}
u_{t}^{\varepsilon}-E \Delta u^{\varepsilon}+u^{\varepsilon}=F\left(u^{\varepsilon}\right), t>0, x \in \Omega \\
E \frac{\partial u^{\varepsilon}}{\partial \vec{n}}=0, t>0, x \in \Gamma
\end{array}\right.
$$

where $u^{\varepsilon}=\left(u_{1}^{\varepsilon}, \ldots, u_{n}^{\varepsilon}\right) \in \mathbb{R}^{n}, E=\operatorname{diag}\left(\varepsilon_{1}, \ldots, \varepsilon_{n}\right)$, with $\varepsilon_{i}>0, i=1, \ldots, n, \vec{n}$ is the outward normal vector to the $\Gamma$ and $\frac{\partial u^{\varepsilon}}{\partial \vec{n}}=\left(\left\langle\nabla u_{1}^{\varepsilon}, \vec{n}\right\rangle, \ldots,\left\langle\nabla u_{n}^{\varepsilon}, \vec{n}\right\rangle\right)$. We assume that the nonlinearity $F: \mathbb{R}^{n} \rightarrow \mathbb{R}^{n}$ is continuous with Lipschitz continuous first derivative and satisfies other hypotheses stated later.

To study (4.8) we need an appropriated functional setting as follows.

Consider the operator $A_{\varepsilon}=\operatorname{diag}\left(A_{1}, \ldots, A_{n}\right)$, where $A_{i}: D\left(A_{i}\right) \subset L^{2}(\Omega) \rightarrow L^{2}(\Omega), i=$ $1, \ldots, n$, is given by

$$
\left\{\begin{array}{l}
D\left(A_{i}\right)=\left\{\varphi \in H^{1}(\Omega): \frac{\partial u}{\partial \vec{n}}=0, \text { in } \Gamma\right\} \\
A_{i} \varphi=-\varepsilon_{i} \Delta \varphi+\varphi
\end{array}\right.
$$

The phase space for partial differential equation of the above type is generally the Sobolev space $H^{1}\left(\Omega, \mathbb{R}^{n}\right)$ (see (BREZIS, 2010)). But since we have the diffusion coefficient as the parameter $\varepsilon$ is natural to consider in $H^{1}\left(\Omega, \mathbb{R}^{n}\right)$ a metric with weight, in this way we will use fractional power spaces of sectorial operators (see (HENRY, 1980)). They play a key role in the theory of the existence of solutions to nonlinear partial differential equations of the parabolic type and in the analysis of asymptotic behavior of solutions for these problems, see Section 2.2 in the Chapter 2. We will require special symmetries and uniformity in the phenomena being modelled in Hilbert spaces. This framework is used frequently because their analysis is relatively 
simple. It is worth mentioning that the above problem can be formulated in more general spaces than $H^{1}(\Omega, \mathbb{R})$, see (CARVALHO; PRIMO, 2004) for a formulation in scale of Banach spaces.

For $i=1, \ldots, n$, let $A_{i}^{\frac{1}{2}}$ denote the fractional power space of $A_{i}$ and $X_{i}^{\frac{1}{2}}$ denote $D\left(A_{i}^{\frac{1}{2}}\right)$ endowed with the graph norm according to (HENRY, 1980), we have

$$
D\left(A_{\varepsilon}^{\frac{1}{2}}\right)=D\left(A_{1}^{\frac{1}{2}}\right) \times \cdots \times D\left(A_{n}^{\frac{1}{2}}\right)=H^{1}\left(\Omega, \mathbb{R}^{n}\right) .
$$

Thus we denote $X_{\varepsilon}^{\frac{1}{2}}=H^{1}\left(\Omega, \mathbb{R}^{n}\right)$ with the inner product given by

$$
\langle\varphi, \psi\rangle_{X_{\varepsilon}^{\frac{1}{2}}}=\int_{\Omega} \varepsilon \nabla \varphi \nabla \psi d x+\int_{\Omega} \varphi \psi d s, \quad \varphi, \psi \in X_{\varepsilon}^{\frac{1}{2}}
$$

and we write (4.8) in the abstract parabolic form

$$
\left\{\begin{array}{l}
u_{t}^{\varepsilon}+A_{\varepsilon} u^{\varepsilon}=f\left(u^{\varepsilon}\right), t>0 \\
u^{\varepsilon}(0)=u_{0}^{\varepsilon} \in X_{\varepsilon}^{\frac{1}{2}}
\end{array}\right.
$$

where $f: X_{\mathcal{\varepsilon}}^{\frac{1}{2}} \rightarrow L^{2}\left(\Omega, \mathbb{R}^{n}\right)$ denotes the Neminskii's functional of $F$.

To obtain the well posedness of the reaction-diffusion equation (4.8) and the existence of global attractors for parabolic equation (4.12), we need to impose some growth and dissipativeness conditions, these conditions are statement in (ARRIETA; CARVALHO; RODRíGUEZBERNAL, 1999; ARRIETA; CARVALHO; RODRíGUEZ-BERNAL, 2000a) and (CARVALHO; LANGA; ROBINSON, 2010).

(i) Growth condition. If $n=2$, for all $\eta>0$, there is a constant $C_{\eta}>0$ such that

$$
|f(u)-f(v)| \leq C_{\eta}\left(e^{\eta|u|^{2}}+e^{\eta|v|^{2}}\right)|u-v|, \quad \forall u, v \in \mathbb{R}
$$

and if $n \geq 3$, there is a constant $\tilde{C}>0$ such that

$$
|f(u)-f(v)| \leq \tilde{C}|u-v|\left(|u|^{\frac{4}{n-2}}+|v|^{\frac{4}{n-2}}+1\right), \quad \forall u, v \in \mathbb{R} .
$$

(ii) Dissipativeness condition

$$
\limsup _{|u| \rightarrow \infty} \frac{f(u)}{u}<0 .
$$

The theory of well-posedness of abstract parabolic problems that enable us to study (4.12) is developed in (CARVALHO; LANGA; ROBINSON, 2010). Results in local well-posedness in the energy space $X_{\varepsilon}^{\frac{1}{2}}$ are obtained due the fact that $A_{\varepsilon}$ generates a strong continuous semigroup and in addition $f$ is continuously differentiable satisfying the above growth condition (i). To show that all solutions of (4.12) are globally defined, we need to impose the above dissipativeness condition (ii). Thus, for each initial date $u_{0}^{\varepsilon}$ in $X_{\varepsilon}^{\frac{1}{2}}$, the equation (4.12) has a global solution through $u_{0}^{\varepsilon}$. This solution is continuously differentiable with respect to the initial data and it is a 
classical solution for $t>0$ satisfying the variation of constants formula. Moreover (4.12) has a global attractor uniformly bounded.

Thus we assume the existence of the solutions $u^{\varepsilon}\left(t, u_{0}^{\varepsilon}\right)$ of (4.12) through $u_{0}^{\varepsilon} \in X_{\varepsilon}^{\frac{1}{2}}$ for positive time and the nonlinear semigroup $T_{\mathcal{\varepsilon}}(t) u_{0}^{\varepsilon}=u^{\varepsilon}\left(t, u_{0}^{\varepsilon}\right)$ satisfies the variation of constants formula given by

$$
T_{\varepsilon}(t) u_{0}^{\varepsilon}=e^{-A_{\varepsilon} t} u_{0}^{\varepsilon}+\int_{0}^{t} e^{-A_{\varepsilon}(t-s)} f\left(T_{\varepsilon}(s) u_{0}^{\varepsilon}\right) d s, t>0
$$

and has a global attractor $\mathscr{A}_{\varepsilon} \subset X_{\varepsilon}^{\frac{1}{2}}$ uniformly bounded, that is,

$$
\sup _{u \in \mathscr{A}_{\mathcal{E}}}\|u\|_{L^{\infty}\left(\Omega, \mathbb{R}^{n}\right)} \leq K
$$

for some constant $K$ independent of $\varepsilon$ (see (ARRIETA; CARVALHO; RODRíGUEZ-BERNAL, 2000a)).

In what follows we denote $L^{2}=L^{2}\left(\Omega, \mathbb{R}^{n}\right)$.

Once the problem is is well posed in the energy space $X_{\mathcal{E}}^{\frac{1}{2}}$, we start to obtain the ordinary differential equation that will describe the asymptotic behaviour of (4.8). For the end we take $\delta>0$ sufficiently small and define the spectral projection $Q_{\varepsilon}: L^{2} \rightarrow L^{2}$, given by

$$
Q_{\varepsilon}=\frac{1}{2 \pi i} \int_{|\xi+1|=\delta}\left(\xi+A_{\varepsilon}\right)^{-1} d \xi
$$

where the eigenspace $Q_{\varepsilon} X_{\varepsilon}^{\frac{1}{2}}$ is isomorphic to $\mathbb{R}^{n}$. The operator $A_{\varepsilon}$ has compact resolvent and $1 \in \sigma\left(A_{\varepsilon}\right)$ is its first eigenvalue, thus $Q_{\varepsilon}$ is well defined projection with finit rank (see (KATO, 1980)). In fact $Q_{\varepsilon} X_{\varepsilon}^{\frac{1}{2}}=\operatorname{span}\left[\varphi_{1}^{\varepsilon}\right]$, where $\varphi_{1}^{\varepsilon}$ is the first eigenfunction of $A_{\varepsilon}$.

With the aid of the projection $Q_{\varepsilon}$ we can decompose the phase space $X_{\varepsilon}^{\frac{1}{2}}$ in a finitedimensional subspace and its complement. This decomposition will allow us to decompose the operator $A_{\varepsilon}$ in order to obtain estimates for the linear semigroup $e^{-A_{\varepsilon} t}$ restricted to these spaces in the decomposition.

Lemma 4.2.1. Let $Q_{\varepsilon}$ be the spectral projection defined in (4.13). If we denote $Y_{\varepsilon}=Q_{\varepsilon} X_{\varepsilon}^{\frac{1}{2}}$ and $Z_{\varepsilon}=\left(I-Q_{\varepsilon}\right) X_{\varepsilon}^{\frac{1}{2}}$ and define the projected operators

$$
A_{\varepsilon}^{+}=\left.A_{\varepsilon}\right|_{Y_{\varepsilon}} \quad \text { and } \quad A_{\varepsilon}^{-}=\left.A_{\varepsilon}\right|_{Z_{\varepsilon}}
$$

then the following estimates are valid,

(i) $\left\|e^{-A_{\varepsilon}^{-} t} z\right\|_{X_{\varepsilon}^{\frac{1}{2}}} \leq M e^{-\left(\varepsilon \lambda_{1}+1\right) t}\|z\|_{X_{\varepsilon}^{\frac{1}{2}}}, \quad t>0, \quad z \in Z_{\varepsilon}$,

(ii) $\left\|e^{-A_{\varepsilon}^{-} t} z\right\|_{X_{\varepsilon}^{\frac{1}{2}}} \leq M e^{-\left(\varepsilon \lambda_{1}+1\right) t} t^{-\frac{1}{2}}\|z\|_{L^{2}}, \quad t>0, \quad z \in Z_{\varepsilon}$ 
where $-\lambda_{1}$ is the first nonzero eigenvalue of the Laplacian with homogeneous Neumann boundary conditions on $\Omega$ and $M$ is a constant independent of $\varepsilon$.

Proof. The operator $A_{\varepsilon}$ is positive self adjoint operator. If we denote its ordered spectrum $\sigma\left(A_{\varepsilon}\right)=\left\{1<\lambda_{2}^{\varepsilon}<\ldots\right\}$ and $\left\{\varphi_{1}^{\varepsilon}, \varphi_{2}^{\varepsilon}, \ldots\right\}$ the associated eigenfunctions, for $z \in Z_{\varepsilon}$ we have

$$
e^{-A_{\varepsilon}^{-} t} z=e^{-A_{\varepsilon} t}\left(I-Q_{\varepsilon}\right) z=\sum_{i=2}^{\infty} e^{-\lambda_{i}^{\varepsilon} t}\left\langle z, \varphi_{i}^{\varepsilon}\right\rangle_{L^{2}} \varphi_{i}^{\varepsilon}, \quad t>0
$$

but $\lambda_{2}^{\varepsilon}<\lambda_{i}^{\varepsilon}$ implies $e^{-\lambda_{i}^{\varepsilon} t}<e^{\lambda_{2}^{\varepsilon} t}$ for $t>0$. Thus

$$
\left\|e^{-A_{\varepsilon}^{-} t} z\right\|_{X_{\varepsilon}^{\frac{1}{2}}} \leq\left(e^{-2 \lambda_{2}^{\varepsilon} t} \sum_{i=2}^{\infty}\left\langle z, \varphi_{i}^{\varepsilon}\right\rangle_{L^{2}}^{2} \lambda_{i}^{\varepsilon}\right)^{\frac{1}{2}} \leq M e^{-\lambda_{2}^{\varepsilon} t}\|z\|_{X_{\varepsilon}^{\frac{1}{2}}}, \quad t>0 .
$$

The function $f(\mu)=e^{-2 \mu t} \mu$ attains its maximum at $\mu=1 / 2 t, t>0$. Then,

$$
\left\|e^{-A_{\varepsilon}^{-} t} z\right\|_{X_{\varepsilon}^{\frac{1}{2}}} \leq\left\{\begin{array}{l}
e^{-\lambda_{2}^{\varepsilon} t}\left(\lambda_{2}^{\varepsilon}\right)^{\frac{1}{2}}\|z\|_{L^{2}}, 1 / 2 t<\lambda_{2}^{\varepsilon} \\
e^{-\lambda_{2}^{\varepsilon} t} 2^{-\frac{1}{2}} t^{-\frac{1}{2}}\|z\|_{L^{2}}, 1 / 2 t>\lambda_{2}^{\varepsilon}
\end{array}\right.
$$

The result follows by noting that $\lambda_{2}^{\varepsilon}=\varepsilon \lambda_{1}+1$.

We can assume, without loss of generality, that $|\Omega|=1$. For the decomposition $X_{\varepsilon}^{\frac{1}{2}}=$ $Y_{\varepsilon} \oplus Z_{\varepsilon}$ we have $Y_{\varepsilon} \approx \mathbb{R}^{n}$ and $Z_{\varepsilon}=\left\{\varphi \in X_{\varepsilon}^{\frac{1}{2}}:\langle\psi, \varphi\rangle_{L^{2}}=0, \psi \in Y_{\varepsilon}\right\}$, with

$$
\langle\psi, \varphi\rangle_{L^{2}}=\int_{\Omega} \varphi(x) \psi(x) d x, \quad \varphi \in Y_{\varepsilon}, \psi \in Z_{\varepsilon} .
$$

Since $\psi$ is a constant map, then $\psi \in L^{\infty}\left(\Omega, \mathbb{R}^{n}\right)$ and thus the above integral is well defined for $\varphi \in X_{\varepsilon}^{\frac{1}{2}}$. Hence if $u(t, \cdot) \in X_{\mathcal{\varepsilon}}^{\frac{1}{2}}$, it can be written as $u(t, x)=v(t)+w(t, x)$, where $v \in Y_{\varepsilon}$ and $w \in Z_{\varepsilon}$ satisfy

$$
v(t)=\int_{\Omega} u(t, x) d x \quad \text { and } \quad \int_{\Omega} w(t, x) d x=0, t>0 .
$$

Thus

$$
\begin{aligned}
\dot{v}(t) & =\int_{\Omega} u_{t}(t, x) d x=\int_{\Omega} E \Delta u(t, x)-u(t, x) d x+\int_{\Omega} F(u(t, x)) d x \\
& =-v(t)+\int_{\Omega} F(v(t)+w(t, x)) d x
\end{aligned}
$$

and

$$
\begin{aligned}
w_{t}(t, x) & =u_{t}(t, x)-\dot{v}(t) \\
& =E \Delta u(t, x)-u(t, x)+F(u(t, x))-\int_{\Omega} F(v(t)+w(t, x)) d x+v(t) \\
& =E \Delta w(t, x)-w(t, x)+F(v(t)+w(t, x))-\int_{\Omega} F(v(t)+w(t, x)) d x .
\end{aligned}
$$


Therefore we can write every solution of (4.8) as a solution of the problem

$$
\left\{\begin{array}{l}
\dot{v}+v=S(v, w), t>0 \\
w_{t}-E \Delta w+w=Q(v, w), t>0, x \in \Omega \\
E \frac{\partial w}{\partial \vec{n}}=0, t>0, x \in \Gamma \\
w(0)=w_{0} \in Z_{\varepsilon}
\end{array}\right.
$$

where

$$
\left\{\begin{array}{l}
S(v, w)=\int_{\Omega} F(v+w) d x, \quad v \in Y_{\varepsilon}, w \in Z_{\varepsilon}, \\
Q(v, w)=F(v+w)-\int_{\Omega} F(v+w) d x, \quad v \in Y_{\varepsilon}, w \in Z_{\varepsilon} .
\end{array}\right.
$$

It is expected that for $\varepsilon$ sufficiently large the part $w(t, x)$ in (4.14) will not play an important role in the asymptotic behavior and in that case the limiting equation should simply be

$$
\dot{u}^{\infty}(t)+u^{\infty}(t)=F\left(u^{\infty}(t)\right)
$$

In fact, the next Theorem inspired by the Theorem 1.1 in (HALE, 1986) shows that $w(t, x)$ and $g(t, v+w)=F(v+w)-S(v, w)=Q(v, w)$ goes to zero exponentially as $t$ goes to infinity in the energy space $X_{\varepsilon}^{\frac{1}{2}}$ when $\varepsilon$ is sufficiently large.

Theorem 4.2.2. Let $S$ and $Q$ as in the definition (4.15). Then there is a positive constant $C$ independent of $\varepsilon$ such that

$$
\|Q(v(t), w(t))\|_{L^{2}} \leq C e^{-\left(\varepsilon \lambda_{1}+1-\mu\right) t} \quad \text { and } \quad\|w(t)\|_{Z_{\varepsilon}} \leq C e^{-\left(\varepsilon \lambda_{1}+1-\mu\right) t},
$$

where $\mu=\left(2 M \Gamma\left(\frac{1}{2}\right)\right)^{\frac{1}{2}}$ and $M$ is given by the Lemma 4.2.1.

Proof. Note that $S(v, 0)=F(v), Q(v, 0)=0$ and $S, Q$ are continuously differentiable with $Q_{v}(0,0)=0=S_{v}(0,0)$, thus there is $\rho>0$ such that for $v_{\varepsilon}, \tilde{v}_{\varepsilon} \in Y_{\varepsilon}$ and $w_{\varepsilon}, \tilde{w}_{\varepsilon} \in Z_{\varepsilon}$,

$$
\begin{aligned}
& \|Q(v, w)\|_{L^{2}} \leq \rho \\
& \|Q(v, w)-Q(\tilde{v}, \tilde{w})\|_{L^{2}} \leq \rho\left(\|z-\tilde{z}\|_{Y_{\varepsilon}}+\|w-\tilde{w}\|_{Z_{\varepsilon}}\right) .
\end{aligned}
$$

Thus

$$
\|Q(v, w)\|_{L^{2}} \leq \rho\|w\|_{Z_{\varepsilon}}, \quad v \in Y_{\varepsilon}, w_{\varepsilon} \in Z_{\varepsilon} .
$$

Hence we just need to estimate $\|w\|_{Z_{\varepsilon}}$.

We use the variation of constants formula to write

$$
w(t)=e^{-A_{\varepsilon}^{-} t} w_{0}+\int_{0}^{t} e^{-A_{\varepsilon}^{-}(t-s)} Q(v(s), w(s)) d s .
$$

Using the estimates from the Lemma 4.2.1, we have

$$
e^{\left(\varepsilon \lambda_{1}+1\right) t}\|w(t)\|_{Z_{\varepsilon}} \leq M\left\|w_{0}\right\|_{Z_{\varepsilon}}+M \int_{0}^{t}(t-s)^{-\frac{1}{2}} e^{\left(\varepsilon \lambda_{1}+1\right) s}\|w(s)\|_{Z_{\varepsilon}} d s,
$$


and by Gronwall's inequality (see (CARVALHO; LANGA; ROBINSON, 2010) pag 168), we obtain for $\mu=\left(2 M \Gamma\left(\frac{1}{2}\right)\right)^{\frac{1}{2}}$,

$$
\|w(t)\|_{Z_{\varepsilon}} \leq 2 M\left\|w_{0}\right\|_{Z_{\varepsilon}} e^{-\left(\varepsilon \lambda_{1}+1-\mu\right) t}
$$

Now we rewrite the ordinay differential equation in (4.14) as $\dot{v}+v=F(v)+[S(v, w)-$ $F(v)$ ], it follows from Theorem 4.2.2 that for $\varepsilon=\min \left\{\varepsilon_{1}, \ldots, \varepsilon_{n}\right\}$ sufficiently large, the asymptotic behavior of (4.8) is determined by the ordinary differential equation (4.16). That is, if $\varepsilon \lambda_{1}>\mu-1$ then the solution $u\left(t, u_{0}\right)$ of the problem (4.8) through $u_{0} \in X_{\varepsilon}^{\frac{1}{2}}$ at $t=0$ satisfies

$$
\left\|u\left(t, u_{0}\right)-v(t)\right\|_{X_{\varepsilon}^{\frac{1}{2}}} \leq K e^{-\left(\varepsilon \lambda_{1}+1-\mu\right) t} \stackrel{t \rightarrow \infty}{\longrightarrow} 0
$$

where $v(t)$ is the average of $u\left(t, u_{0}\right)$ in $\Omega$. Thus, if we assume that the equation (4.16) has a global attractor $\mathscr{A}_{\infty} \subset \mathbb{R}^{n}$ and understand $\mathbb{R}^{n}$ as the subspace of constant functions in $X_{\varepsilon}^{\frac{1}{2}}$, we have $A_{\infty}$ a compact subset in $X_{\varepsilon}^{\frac{1}{2}}$ invariant under $T_{\mathcal{E}}(\cdot)$ and it follows from (4.17) that $\mathscr{A}_{\infty}$ attracts under $T_{\varepsilon}(\cdot)$ bounded set in $X_{\mathcal{\varepsilon}}^{\frac{1}{2}}$, hence $\mathscr{A}_{\infty}=\mathscr{A}_{\varepsilon}$, when $\varepsilon \lambda_{1}>\mu-1$.

In what folows we will consider $\varepsilon \in\left[m_{0}, \bar{\mu}\right]$, for some $m_{0}>0$ and $\bar{\mu}>(\mu-1) \lambda_{1}^{-1}$. It is clear that $\mathscr{A}_{\varepsilon}=\mathscr{A}_{\infty}$ for $\varepsilon \geq \bar{\mu}$, thus we are concerning in what happens when $\varepsilon$ approaches $\bar{\mu}$ to the left. We will see that the family of attractors $\left\{\mathscr{A}_{\varepsilon}\right\}$ with $\varepsilon \in\left[m_{0}, \bar{\mu}\right]$ is continuous as $\varepsilon \rightarrow \bar{\mu}$ and this continuity can be estimated by a rate of convergence given by $\varepsilon^{-\frac{1}{2}}$ that goes to zero when $\varepsilon$ goes to infinity. Since $Y_{\varepsilon}$ is isomorphic to $\mathbb{R}^{n}$ and their norms are uniformly equivalent (by (4.11)) we will consider $Y_{\varepsilon}=\mathbb{R}^{n}$.

In order to obtain estimate to the convergence of the attractor $\mathscr{A}_{\varepsilon}$ of the equation (4.12) to the attractor $\mathscr{A}_{\infty}$ of the (4.16) as $\varepsilon \rightarrow \bar{\mu}$ following the results of the (SANTAMARIA; ARRIETA, 2014), we assume the nonlinear semigroup $T_{\infty}(\cdot)$ generated by solutions of the (4.16) is a Morse-Smale semigroup in $\mathbb{R}^{n}$. More precisely,

$$
T_{\infty}(t) u_{0}^{\infty}=e^{-A_{\infty} t} u_{0}^{\infty}+\int_{0}^{t} e^{-A_{\infty}(t-s)} F\left(T_{\infty}(s) u_{0}^{\infty}\right) d s, t>0, u_{0}^{\infty} \in \mathbb{R}^{n},
$$

where $A_{\infty}=I$ denote the identity in $\mathbb{R}^{n}$ and if we denote $\mathscr{E}_{\infty}$ the set of its equilibrium points, then it is composed of $p$ hyperbolic points, that is,

$$
\mathscr{E}_{\infty}=\left\{\varphi \in \mathbb{R}^{n}: A_{\infty} \varphi-F(\varphi)=0\right\}=\left\{u_{1}^{\infty, *}, \ldots, u_{p}^{\infty, *}\right\}
$$

where the spectrum set $\sigma\left(A_{\infty}-F^{\prime}\left(u_{i}^{\infty, *}\right)\right) \cap\left\{\varphi \in \mathbb{R}^{n}:\|\varphi\|_{\mathbb{R}}=1\right\}=\emptyset, i=1, \ldots, p$. Moreover, $T_{\infty}(\cdot)$ is dynamically gradient (see (CARVALHO; LANGA; ROBINSON, 2010)),

$$
\mathscr{A}_{\infty}=\bigcup_{i=1}^{p} W^{u}\left(u_{i}^{\infty, *}\right),
$$


where $W^{u}\left(u_{i}^{\infty, *}\right)$ is the unstable manifold associated with the equilibrium point in $\mathscr{E}_{\infty}$ and for $i \neq j$ the unstable manifold $W^{u}\left(u_{i}^{\infty, *}\right)$ and the local stable manifold $W_{\text {loc }}^{s}\left(u_{j}^{\infty, *}\right)$ has transversal intersection, see (BORTOLAN et al., 2015).

Similarly to hyperbolicity the assumption that $T_{\infty}(\cdot)$ is a Morse-Smale semigroup is not too restrictive, in fact is proved in (BRUNOVSKÝ; POLÁCIK, 1997) that this situation is generic.

We will study the problem (4.8) as a small perturbation of (4.16) and the continuity of attractors will be considered, in fact, the assumptions above enable us to obtain the geometric equivalence of phase diagrams when $\varepsilon$ approaches $\bar{\mu}$. This property is known as geometric structural stability and it is the main feature of Morse-Smale systems. In this way we are under the conditions described in the Chapter 3 where results about rate of convergence of attractor for Morse-Smale problems were obtained.

In what follows we prove the convergence of the resolvent operators and we obtain estimates for the convergence of the analitic linear semigroup generated by $-A_{\varepsilon}$. We establish that the rate for these convergence is $\varepsilon^{-\frac{1}{2}}$.

We saw that the operators $A_{\varepsilon}$ and $A_{\infty}$ work in different spaces. In fact the operator $A_{\infty}$ is the identity in $\mathbb{R}^{n}$ that can be understood as the space of constant functions in $X_{\mathcal{\varepsilon}}^{\frac{1}{2}}$. Thus we need to find a way to compare functions between these spaces. The abstract theory that can be used to compare linear problems in different spaces is developed in (CARVALHO; PISKAREV, 2006) and named E-convergence, see Chapter 3. In this context we consider, as in the previous section, the inclusion operator $i: \mathbb{R}^{n} \rightarrow X_{\mathcal{\varepsilon}}^{\frac{1}{2}}$ and the projection $P: X_{\mathcal{\varepsilon}}^{\frac{1}{2}} \rightarrow \mathbb{R}^{n}$ given by

$$
P u=\frac{1}{|\Omega|} \int_{\Omega} u d x, \quad u \in X_{\varepsilon}^{\frac{1}{2}}
$$

Not that $P$ can also be considered as an orthogonal projection acting on $L^{2}$ onto $\mathbb{R}^{n}$.

The operator $A_{\varepsilon}$ is, for each $\varepsilon$, an invertible operator with compact resolvent. The next result ensure that the resolvent operator approaches the projection $P$ uniformly in the operator norm.

Lemma 4.2.3. For $g \in L^{2}\left(\Omega, \mathbb{R}^{n}\right)$ such that $\|g\|_{L^{2}\left(\Omega, \mathbb{R}^{n}\right)} \leq 1$, let $u^{\varepsilon}$ be the weak solution of the elliptic problem $A_{\varepsilon} u^{\varepsilon}=g$. Then there is a positive constant $C$ independent of $\varepsilon$ such that

$$
\left\|u^{\varepsilon}-u^{\infty}\right\|_{X_{\varepsilon}^{\frac{1}{2}}} \leq C \varepsilon^{-\frac{1}{2}}
$$

where $u^{\infty}=P g$.

Proof. We denote $u^{\varepsilon}=\left(u_{1}^{\varepsilon}, \ldots, u_{n}^{\varepsilon}\right)$ and $g=\left(g_{1}, \ldots, g_{n}\right)$, for $i=1, \ldots, n$. Since (4.10) holds we can only consider one component $u_{i}^{\varepsilon}$. Then

$$
\int_{\Omega} \varepsilon_{i} \nabla u_{i}^{\varepsilon} \nabla \varphi d x+\int_{\Omega} u_{i}^{\varepsilon} \varphi d x=\int_{\Omega} g_{i} \varphi d x, \quad \varphi \in H^{1}(\Omega) ;
$$




$$
\int_{\Omega} u_{i}^{\infty} \psi d x=\int_{\Omega} P g_{i} \psi d x, \quad \psi \in \mathbb{R}
$$

Thus

$$
\begin{gathered}
\int_{\Omega} \varepsilon_{i}\left|\nabla u_{i}^{\varepsilon}\right|^{2} d x+\int_{\Omega} u_{i}^{\varepsilon}\left(u_{i}^{\varepsilon}-u_{i}^{\infty}\right) d x=\int_{\Omega} g_{i}\left(u_{i}^{\varepsilon}-u_{i}^{\infty}\right) d x \\
\int_{\Omega} u_{i}^{\infty}\left(P u_{i}^{\varepsilon}-u_{i}^{\infty}\right) d x=\int_{\Omega} P g_{i}\left(P u_{i}^{\varepsilon}-u_{i}^{\infty}\right) d x
\end{gathered}
$$

which implies

$$
\int_{\Omega} g_{i}\left(u_{i}^{\varepsilon}-u_{i}^{\infty}\right) d x-\int_{\Omega} P g_{i}\left(P u_{i}^{\varepsilon}-u_{i}^{\infty}\right) d x=\int_{\Omega} g_{i}(I-P) u_{i}^{\varepsilon} d x
$$

and

$$
\int_{\Omega} \varepsilon_{i}\left|\nabla u_{i}^{\varepsilon}\right|^{2} d x+\int_{\Omega} u_{i}^{\varepsilon}\left(u_{i}^{\varepsilon}-u_{i}^{\infty}\right) d x-\int_{\Omega} u_{i}^{\infty}\left(P u_{i}^{\varepsilon}-u_{i}^{\infty}\right) d x=\left\|u_{i}^{\varepsilon}-u_{i}^{\infty}\right\|_{X_{i}^{\frac{1}{2}}}^{2}
$$

Therefore

$$
\left\|u_{i}^{\varepsilon}-u_{i}^{\infty}\right\|_{X_{i}^{\frac{1}{2}}}^{2} \leq \int_{\Omega}\left|g_{i}(I-P) u_{i}^{\varepsilon}\right| d x .
$$

By Poincaré's inequality for average, we have

$$
\int_{\Omega}\left|g_{i}(I-P) u_{i}^{\varepsilon}\right| d x \leq\left\|g_{i}\right\|_{L^{2}}\left(\int_{\Omega}\left|\nabla u_{i}^{\varepsilon}\right|^{2} d x\right)^{\frac{1}{2}},
$$

but

$$
\varepsilon \int_{\Omega}\left|\nabla u_{i}^{\varepsilon}\right|^{2} d x \leq\left\|u_{i}^{\varepsilon}-u_{i}^{\infty}\right\|_{X_{i}^{\frac{1}{2}}}^{2}
$$

Put this estimates together we obtain (4.18).

Remark 4.2.4. When we work with large diffusion the norm in $X_{\varepsilon}^{\frac{1}{2}}$ in general is equivalent to the norm of $H^{1}$ but this equivalence is not uniform, indeed it follows from (4.11) the inequalities

$$
m_{0}\|u\|_{H^{1}}^{2} \leq\|u\|_{X_{\varepsilon}^{\frac{1}{2}}}^{2} \leq \varepsilon\|u\|_{H^{1}}^{2}
$$

Hence estimates in the Sobolev spaces $H^{1}$ does not give suitable estimates in the half fractional power space $X_{\varepsilon}^{\frac{1}{2}}$. Of course, as we are considering $\varepsilon \in\left[m_{0}, \bar{\mu}\right]$ we can obtain uniform estimate in the inequality above by limiting $\varepsilon \leq \bar{\mu}$, but we do not do this here because there are many problems, as we saw in the previous section, where the diffusion runs an unbounded interval and the limiting problem is obtained in the limit process $\varepsilon \rightarrow \infty$.

Note that by Poincare's inequality we can obtain a better estimate if we work in $H^{1}$, that is, $\left\|u^{\varepsilon}-u^{\infty}\right\|_{H^{1}} \leq C \varepsilon^{-1}$, for some constant $C$ independent of $\varepsilon$.

Hence it is clear that due the non-uniformity in the norms we have some lost when we consider $X_{\mathcal{E}}^{\frac{1}{2}}$-norm than $H^{1}$-norm. This can be seen in the following example. 
Example 4.2.5. Consider the one-dimensional elliptic problem

$$
\left\{\begin{array}{l}
-\varepsilon u_{x x}=\cos (2 \pi x), x \in(0,1), \\
u_{x}(0)=0=u_{x}(1)
\end{array}\right.
$$

We have $u^{\varepsilon}(x)=\frac{1}{\varepsilon} \frac{\cos (2 \pi x)}{4 \pi^{2}}$ and $u^{\infty}=0$. Thus

$$
\left\|u^{\varepsilon}-u^{\infty}\right\|_{X_{\varepsilon}^{\frac{1}{2}}}^{2}=\int_{0}^{1} \varepsilon\left|\frac{1}{\varepsilon} \frac{\sin (2 \pi x)}{4 \pi^{2}}\right|^{2} d x=C \varepsilon^{-1},
$$

where $C$ is a constant independent of $\varepsilon$.

The Lemma 4.2.3 determines the natural quantity that will be used to study the convergence of the dynamic of the problem (4.8) when $\varepsilon$ approaches $\bar{\mu}$. The rate of convergence is given by $\varepsilon^{-\frac{1}{2}}$ that goes to zero as $\varepsilon$ goes to infinity. In fact, if we denote $u^{\varepsilon}=A_{\varepsilon}^{-1} g$ then $u^{\varepsilon}$ is the weak solution of the elliptic problem $A_{\varepsilon} u^{\varepsilon}=g$ and since $g$ is an arbitrary map in $L^{2}$, we obtain

$$
\left\|A_{\varepsilon}^{-1}-P\right\|_{\mathscr{L}\left(L^{2}, X_{\varepsilon}^{\frac{1}{2}}\right)} \leq C \varepsilon^{\frac{1}{2}}
$$

This estimate agrees with the compact convergence in (CARVALHO; PISKAREV, 2006) and (CARVALHO et al., 2010), that is the operator $A_{\varepsilon}^{-1}$ converges compactly to $A_{\infty}^{-1} P=P$.

Note that, if we take $\varphi=1$ as a test function in (4.19), we have $u^{\infty}=P u^{\varepsilon}$, hence (4.18) ensures that $u^{\varepsilon}$ converge for its average in $X_{\mathcal{\varepsilon}}^{\frac{1}{2}}$ and this rate of convergence is $\varepsilon^{-\frac{1}{2}}$.

Now we will see how the converge of the resolvent operators implies the convergence of the eigenvalues and spectral projections defined in (4.13). We have

$$
\left\|Q_{\varepsilon}-P\right\|_{\mathscr{L}\left(L^{2}, X_{\varepsilon}^{\frac{1}{2}}\right)} \leq \frac{1}{2 \pi} \int_{|\xi+1|=\delta}\left\|\left(\xi+A_{\varepsilon}\right)^{-1}-(\xi+I)^{-1} P\right\|_{\mathscr{L}\left(L^{2}, X_{\varepsilon}^{\frac{1}{2}}\right)} d \xi \leq C \varepsilon^{-\frac{1}{2}}
$$

Since $A_{\infty}=I$ in $\mathbb{R}^{n}$ we can denote $Q_{\infty}=I$, in other words, $Q_{\varepsilon}$ converges compactly to $Q_{\infty}^{-1} P=P$. Note that since the operator $A_{\varepsilon}$ has compact resolvent, the spectral projection $Q_{\varepsilon}$ is a compact operator. Thus, for $\varepsilon$ sufficiently large, the eigenspace $W_{\varepsilon}=Q_{\varepsilon} X_{\varepsilon}^{\frac{1}{2}}$ has dimension $\operatorname{dim}\left(W_{\varepsilon}\right)=$ $\operatorname{dim}\left(\mathbb{R}^{n}\right)=n$ (see (KATO, 1980)). Moreover the eigenvalues $\lambda_{i}^{2}, i \geq 2$ goes to infinity as $\varepsilon$ goes to infinity. The last property was used implicitly in the last section when we guessed the limiting ordinary differential equation.

Lemma 4.2.6. Let $A_{\varepsilon}$ the operator defined in (4.9) and let $\sigma\left(A_{\varepsilon}\right)=\left\{1<\lambda_{2}^{\varepsilon}<\lambda_{3}^{\varepsilon}, \ldots\right\}$ its ordered spectrum. Then $\lambda_{j}^{\varepsilon} \rightarrow \infty$ as $\varepsilon \rightarrow \infty$ and $j \geq 2$.

Proof. Assume that there is $R>0$ and there are sequences $\varepsilon_{k} \rightarrow \infty$ as $k \rightarrow \infty$ and $\left\{\lambda_{j}^{\varepsilon_{k}}\right\}_{k}, j \geq 2$, such that, $\lambda_{j}^{\varepsilon_{k}} \in \sigma\left(A_{\varepsilon_{k}}\right)$ and $\left|\lambda_{j}^{\varepsilon_{k}}\right| \leq R$. We can assume $\lambda_{j}^{\varepsilon_{k}} \rightarrow \lambda$. Let $u_{j}^{\varepsilon_{k}}$ be the corresponding eigenfunction to $\lambda_{j}^{\varepsilon_{k}}$ with $\left\|u_{j}^{\varepsilon_{k}}\right\|_{X_{\varepsilon_{k}}^{\frac{1}{2}}}=1$. Then $u_{j}^{\varepsilon_{k}}=\lambda_{j}^{\varepsilon_{k}} A_{\varepsilon_{k}}^{-1} u_{j}^{\varepsilon_{k}}$. Since $A_{\varepsilon_{k}}$ converges compactly 
to $A_{\infty}^{-1} P$, we can assume, see (CARVALHO; LANGA; ROBINSON, 2010), $u_{j}^{\varepsilon_{k}} \rightarrow u$ as $\varepsilon_{k} \rightarrow \infty$ for some $u \in \mathbb{R}^{n}$. Thus

$$
u_{j}^{\varepsilon_{k}}=\lambda_{j}^{\varepsilon_{k}} A_{\varepsilon_{k}}^{-1} u_{j}^{\varepsilon_{k}} \rightarrow \lambda A_{\infty}^{-1} u,
$$

as $\varepsilon_{k} \rightarrow \infty$. Since $u_{j}^{\varepsilon_{k}} \rightarrow u$, we get $u=\lambda A_{\infty}^{-1} u$, which implies $\lambda \in \sigma\left(A_{\infty}\right)$, thus $\lambda=1$ and $\lambda_{j}^{\varepsilon_{k}} \rightarrow 1$ as $\varepsilon_{k} \rightarrow \infty, j \geq 2$, which is absurd.

Now we can state the main result of this section.

Theorem 4.2.7. For $\varepsilon$ sufficiently large there is an invariant manifold $\mathscr{M}_{\varepsilon}$ for (4.12), which is given by graph of a certain Lipschitz continuous map $s_{*}^{\varepsilon}: \mathbb{R}^{n} \rightarrow Z_{\varepsilon}$ as

$$
\mathscr{M}_{\varepsilon}=\left\{u^{\varepsilon} \in X_{\varepsilon}^{\frac{1}{2}} ; u^{\varepsilon}=Q_{\varepsilon} u^{\varepsilon}+s_{*}^{\varepsilon}\left(Q_{\varepsilon} u^{\varepsilon}\right)\right\}
$$

The map $s_{*}^{\varepsilon}: \mathbb{R}^{n} \rightarrow Z_{\varepsilon}$ satisfies the condition

$$
\left\|s_{*}^{\varepsilon}\right\|\left\|=\sup _{v^{\varepsilon} \in \mathbb{R}^{n}}\right\| s_{*}^{\varepsilon}\left(v^{\varepsilon}\right) \|_{X_{\varepsilon}^{\frac{1}{2}}} \leq C \varepsilon^{-\frac{1}{2}},
$$

for some positive constant $C$ independent of $\varepsilon$. The invariant manifold $\mathscr{M}_{\varepsilon}$ is exponentially attracting and the global attractor $\mathscr{A}_{\varepsilon}$ of the problem (4.12) lying in $\mathscr{M}_{\varepsilon}$ and the flow on $\mathscr{A}_{\varepsilon}$ is given by

$$
u^{\varepsilon}(t)=v^{\varepsilon}(t)+s_{*}^{\varepsilon}\left(v^{\varepsilon}(t)\right), \quad t \in \mathbb{R},
$$

where $v^{\varepsilon}(t)$ satisfy

$$
\dot{v}^{\varepsilon}+A_{\varepsilon}^{+} v^{\varepsilon}=Q_{\varepsilon} f\left(v^{\varepsilon}+s_{*}^{\varepsilon}\left(v^{\varepsilon}(t)\right)\right) .
$$

Moreover, the continuity of the attractors can be estimated by

$$
\mathrm{d}_{\varepsilon}\left(\mathscr{A}_{\varepsilon}, \mathscr{A}_{\infty}\right) \leq C \varepsilon^{-\frac{1}{2}} .
$$

Proof. All conditions of the Chapter 3 are satisfied. 


\section{LARGE DIFFUSION EXCEPT IN A NEIGHBORHOOD OF A POINT}

There are some applications where the diffusion coefficient is large in parts of the domain and becomes small in another parts. In this chapter we consider a class of diffusion coefficients $p_{\varepsilon} \in C^{1}([0,1])$ that are large except in a neighborhood of a finite number of point where they become small. This kind of problem has been studied in the works (CARVALHO; PEREIRA, 1994) and (FUSCO, 1987) where it has proved that the dynamics is dictated by a ordinary differential equation. We obtain a rate of convergence that enable us knowing how fast the dynamics described above approaches an ordinary differential equation when the parameter $\varepsilon$ varies.

\subsection{Functional Setting}

Consider the scalar parabolic problem

$$
\left\{\begin{array}{l}
u_{t}^{\varepsilon}-\left(p_{\varepsilon} u_{x}^{\varepsilon}\right)_{x}+\lambda u^{\varepsilon}=f\left(u^{\varepsilon}\right), \quad 0<x<1, t>0 \\
u_{x}(0)=0=u_{x}(1), \quad t>0
\end{array}\right.
$$

where $\varepsilon \in\left(0, \varepsilon_{0}\right], 0<\varepsilon_{0} \leq 1, \lambda>0$ and $f: \mathbb{R} \rightarrow \mathbb{R}$ is continuously differentiable and satisfies the following dissipativeness condition

$$
\limsup _{|u| \rightarrow \infty} \frac{f(u)}{u} \leq-r
$$


for some $r>0$. Assume that the diffusion is large except in a neighborhood of a point where it becomes small, that is, $p_{\varepsilon} \in C^{2}([0,1])$ and for positive constants $e_{1}, e_{2}, l_{1}$ and $a_{1}$, is valid

$$
\begin{cases}p_{\varepsilon}(x) \geq \frac{e_{1}}{\varepsilon}, & x \in\left[0, x_{1}-\varepsilon l_{1}^{\prime}\right], \\ p_{\varepsilon}(x) \geq \frac{e_{2}}{\varepsilon}, & x \in\left[x_{1}+\varepsilon l_{1}^{\prime}, 1\right], \\ p_{\varepsilon}(x) \geq \varepsilon a_{1}, & x \in\left[x_{1}-\varepsilon l_{1}^{\prime}, x_{1}+\varepsilon l_{1}^{\prime}\right], \\ p_{\varepsilon}(x) \leq \varepsilon a_{1}^{\prime} & x \in\left[x_{1}-\varepsilon l_{1}, x_{1}+\varepsilon l_{1}\right],\end{cases}
$$

where $0<x_{1}<1$ and $l_{1}^{\prime}, a_{1}^{\prime}$ are functions of $\varepsilon$ that approach $l_{1}, a_{1}$ respectively from above as $\varepsilon \rightarrow 0$ (see Figure (10)).

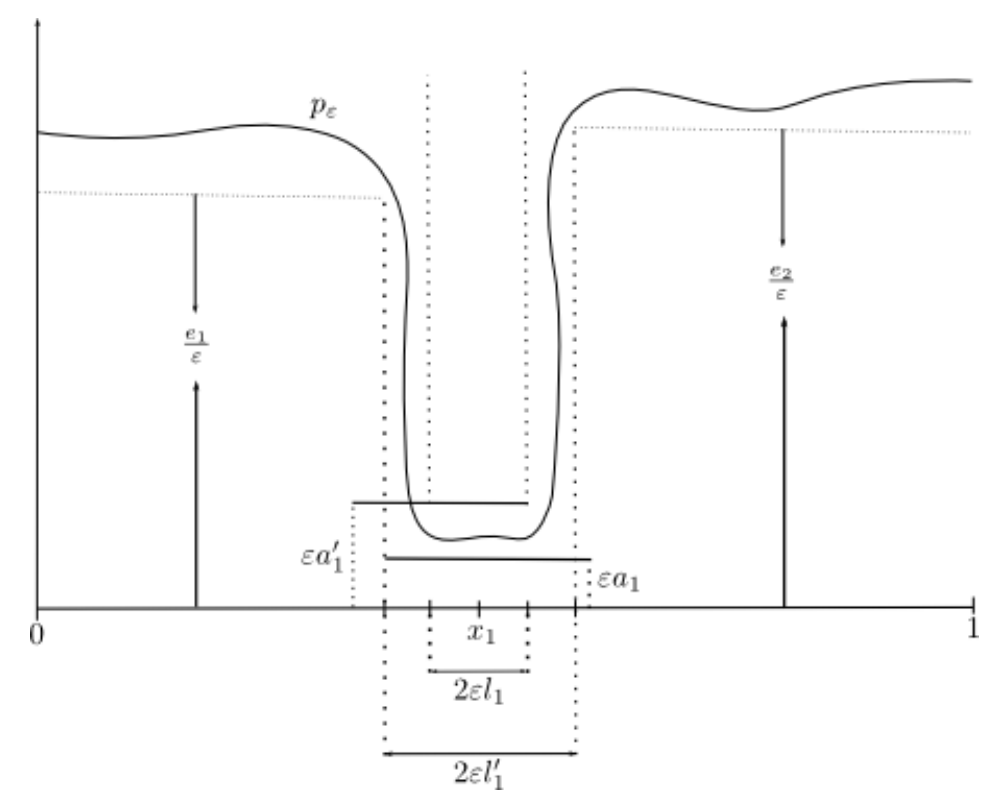

Figure 10 - Diffusion

A more general situation where the diffusion is large except in the neighborhood of $n$ points can be found in (CARVALHO; PEREIRA, 1994). Here, for simplicity, we consider the diffusion large except in the neighborhood of one point. The same approach can be carried out in the general case.

We follow (CARVALHO; PEREIRA, 1994) and (FUSCO, 1987) in order to determine the asymptotic behavior of (5.1). It is expect that the solutions converge to a constant (possibly different) on each of the interval $\left(0, x_{1}\right)$ and $\left(x_{1}, 1\right)$, which implies that the equation (5.1) has the same dynamic as an ordinary differential equation in $\mathbb{R}^{2}$.

We start by defining the operator $A_{\varepsilon}: D\left(A_{\varepsilon}\right) \subset L^{2}(0,1) \rightarrow L^{2}(0,1)$ by

$$
\mathscr{D}\left(A_{\varepsilon}\right)=\left\{u \in H^{2}(0,1) ; u_{x}(0)=u_{x}(1)=0\right\} \quad \text { and } \quad A_{\varepsilon} u=-\left(p_{\varepsilon} u_{x}\right)_{x}+\lambda u .
$$

We have $A_{\varepsilon}$ is a self-adjoint invertible operator with compact resolvent for each $\varepsilon \in\left(0, \varepsilon_{0}\right]$, thus we can define, in the usual way (see(HENRY, 1980)), the fractional power space $X_{\varepsilon}^{\frac{1}{2}}=H^{1}(0,1)$, $\varepsilon \in\left(0, \varepsilon_{0}\right]$. 
The proof of the next Lemma follows the same arguments in the Section 2 in (CARVALHO; PEREIRA, 1994).

Lemma 5.1.1. Let $\sigma\left(A_{\varepsilon}\right)=\left\{\lambda, \lambda_{2}^{\varepsilon}, \lambda_{3}^{\varepsilon}, \ldots\right\}$ be the sequence of eigenvalues of operator $A_{\varepsilon}$ and let $\left\{1, \varphi_{2}^{\varepsilon}, \varphi_{3}^{\varepsilon}, \ldots\right\}$ be the corresponding sequence of normalized eigenfunctions. Then

$$
\lambda_{2}^{\varepsilon} \stackrel{\varepsilon \rightarrow 0}{\longrightarrow} \frac{a_{1}}{2 l_{1} x_{1}\left(1-x_{1}\right)}+\lambda, \quad \lambda \stackrel{\varepsilon}{\stackrel{\varepsilon \rightarrow 0}{\longrightarrow} \infty}, j \geq 3
$$

and

$$
\varphi_{2}^{\varepsilon} \stackrel{\varepsilon \rightarrow 0}{\longrightarrow}\left\{\begin{array}{l}
-\sqrt{\frac{1-x_{1}}{x_{1}}} x \in\left(0, x_{1}\right) \\
\sqrt{\frac{x_{1}}{1-x_{1}}}, x \in\left(x_{1}, 1\right)
\end{array}\right.
$$

We can decompose the fractional power space $X_{\mathcal{\varepsilon}}^{\frac{1}{2}}=\operatorname{span}\left[1, \varphi_{2}^{\varepsilon}\right] \oplus\left(\operatorname{span}\left[1, \varphi_{2}^{\varepsilon}\right]\right)^{\perp}$ which implies that the limiting problem of (5.1) is given by following ordinary differential equation in $\mathbb{R}^{2}$

$$
\left[\begin{array}{c}
\dot{u}_{1}^{0} \\
\dot{u}_{2}^{0}
\end{array}\right]+\left[\begin{array}{cc}
\frac{a_{1}}{2 l_{1} x_{1}}+\lambda & -\frac{a_{1}}{2 l_{1} x_{1}} \\
-\frac{a_{1}}{2 l_{1}\left(1-x_{1}\right)} & \frac{a_{1}}{2 l_{1}\left(1-x_{1}\right)}+\lambda
\end{array}\right]\left[\begin{array}{l}
u_{1}^{0} \\
u_{2}^{0}
\end{array}\right]=\left[\begin{array}{c}
f\left(u_{1}^{0}\right) \\
f\left(u_{2}^{0}\right)
\end{array}\right] \text {. }
$$

Proof. If $\lambda \in \sigma\left(A_{\varepsilon}\right)$ and $\varphi$ is the matching eigenfunction, then

$$
\lambda=\int_{0}^{1} p_{\varepsilon}\left|\varphi^{\prime}\right|^{2} d x+\int_{0}^{1} \lambda|\varphi|^{2} d x
$$

Assume that there is a constant $m$ such that $\lambda \leq m$ uniformly in $\varepsilon$. Thus

$$
\int_{0}^{x_{1}-\varepsilon l_{1}^{\prime}} \frac{e_{1}}{\varepsilon}\left|\varphi^{\prime}\right|^{2} d x \leq \int_{0}^{x_{1}-\varepsilon l_{1}^{\prime}} p_{\varepsilon}|\varphi|^{2} d x \leq m
$$

and

$$
\int_{0}^{x_{1}-\varepsilon l_{1}^{\prime}}\left|\varphi^{\prime}\right|^{2} d x \leq \frac{m}{e_{1}} \varepsilon
$$

This inequality implies that, for $x \in\left[0, x_{1}-\varepsilon l_{1}^{\prime}\right]$, we have

$$
|\varphi(x)-\varphi(0)|=\left|\int_{0}^{x} \varphi^{\prime}(s) d s\right| \leq\left(\int_{0}^{x_{1}-\varepsilon l_{1}^{\prime}}\left|\varphi^{\prime}\right|^{2} d x\right)^{\frac{1}{2}} \leq\left(\frac{m}{e_{1}}\right)^{\frac{1}{2}} \varepsilon^{\frac{1}{2}}
$$

which implies

$$
\varphi(x)=\varphi(0)+O\left(\varepsilon^{\frac{1}{2}}\right), \quad x \in\left[0, x_{1}-\varepsilon l_{1}^{\prime}\right] .
$$

Similarly

$$
\varphi(x)=\varphi(1)+O\left(\varepsilon^{\frac{1}{2}}\right), \quad x \in\left[x_{1}+\varepsilon l_{1}^{\prime}, 1\right] .
$$

For the interval $\left[x_{1}-\varepsilon l_{1}^{\prime}, x_{1}-\varepsilon l_{1}\right]$, we have

$$
\int_{x_{1}-\varepsilon l_{1}^{\prime}}^{x_{1}-\varepsilon l_{1}}\left|\varphi^{\prime}\right|^{2} d x \leq \frac{m}{\varepsilon a_{1}}
$$


therefore

$$
\begin{aligned}
\left|\varphi(x)-\varphi\left(x_{1}-\varepsilon l_{1}^{\prime}\right)\right| & =\left|\int_{x_{1}-\varepsilon l_{1}^{\prime}}^{x} \varphi^{\prime}(s) d s\right| \\
& \leq\left(\int_{x_{1}-\varepsilon l_{1}^{\prime}}^{x_{1}-\varepsilon l_{1}}\left|\varphi^{\prime}\right|^{2} d x\right)^{\frac{1}{2}}\left(\varepsilon l_{1}^{\prime}-\varepsilon l_{1}\right)^{\frac{1}{2}} \\
& \leq\left(\frac{m}{a_{1}}\right)^{\frac{1}{2}}\left(l_{1}-l_{1}^{\prime}\right)^{\frac{1}{2}}
\end{aligned}
$$

and

$$
\varphi(x)=\varphi\left(x_{1}-\varepsilon l_{1}^{\prime}\right)+\left(\frac{m}{a_{1}}\right)^{\frac{1}{2}}\left(l_{1}-l_{1}^{\prime}\right)^{\frac{1}{2}} .
$$

By (5.4) it follows that

$$
\varphi(x)=\varphi(0)+O\left(\left(l_{1}-l_{1}^{\prime}\right)^{\frac{1}{2}}\right), \quad x \in\left[x_{1}-\varepsilon l_{1}^{\prime}, x_{1}-\varepsilon l_{1}\right] .
$$

In the same way,

$$
\varphi(x)=\varphi(1)+O\left(\varepsilon^{\frac{1}{2}}\right)+O\left(\left(l_{1}-l_{1}^{\prime}\right)^{\frac{1}{2}}\right), \quad x \in\left[x_{1}+\varepsilon l_{1}, x_{1}+\varepsilon l_{1}^{\prime}\right] .
$$

Finally, for $x \in\left[x_{1}-\varepsilon l_{1}, x_{1}+\varepsilon l_{1}\right]$, we have

$$
\begin{aligned}
\left|\varphi(x)-\varphi\left(x_{1}-\varepsilon l_{1}\right)\right| & =\left|\int_{x_{1}-\varepsilon l_{1}}^{x} \varphi^{\prime}(s) d s\right| \leq\left(\int_{x_{1}-\varepsilon l_{1}}^{x_{1}+\varepsilon l_{1}}\left|\varphi^{\prime}(x)\right|^{2} d x\right)^{\frac{1}{2}}\left(2 \varepsilon l_{1}\right)^{\frac{1}{2}} \\
& \leq\left(\frac{m}{a_{1}}\right)^{\frac{1}{2}}\left(2 \varepsilon l_{1}\right)^{\frac{1}{2}}
\end{aligned}
$$

since

$$
\int_{x_{1}-\varepsilon l_{1}}^{x_{1}+\varepsilon l_{1}}\left|\varphi^{\prime}(x)\right|^{2} d x \leq \frac{m}{\varepsilon a_{1}} .
$$

This implies that

$$
\left|\varphi(x)-\varphi\left(x_{1}-\varepsilon l_{1}\right)\right| \leq\left(\frac{2 m l_{1}}{a_{1}}\right)^{\frac{1}{2}}
$$

thus

$$
\varphi(x)=\varphi(0)+O(1), \quad x \in\left[x_{1}-\varepsilon l_{1}, x_{1}+\varepsilon l_{1}\right] .
$$

We now prove that $\varphi(0)$ and $\varphi(1)$ are uniformly bounded. Using (5.4) and $\|\varphi\|_{L^{2}}=1$, we obtain

$$
\int_{0}^{x_{1}-\varepsilon l_{1}^{\prime}}\left(\varphi(0)+O\left(\varepsilon^{\frac{1}{2}}\right)\right)^{2} d x \leq 1
$$

hence,

$$
0 \leq \varphi(0)^{2}+\varphi(0) O\left(\varepsilon^{\frac{1}{2}}\right)+O(\varepsilon) \leq \frac{1}{x_{1}-\varepsilon l_{1}^{\prime}},
$$

which proves $\varphi(0)$ is uniformly bounded.

In a similar way, using (5.5) and $\|\varphi\|_{L^{2}}=1$, we obtain $\varphi(1)$ uniformly bounded 
Using the estimate above and the fact that $\varphi$ has average zero, we obtain,

$$
\varphi(0) x_{1}+\varphi(1)\left(1-x_{1}\right)+O\left(\varepsilon^{\frac{1}{2}}\right)=0
$$

and

$$
(\varphi(0))^{2} x_{1}+(\varphi(1))^{2}\left(1-x_{1}\right)+O\left(\varepsilon^{\frac{1}{2}}\right)=1
$$

Solving (5.6) and (5.7), we get

$$
\begin{aligned}
& (\varphi(0))^{2}=\frac{1-x_{1}}{x_{1}}+O\left(\varepsilon^{\frac{1}{2}}\right), \\
& (\varphi(1))^{2}=\frac{x_{1}}{1-x_{1}}+O\left(\varepsilon^{\frac{1}{2}}\right) .
\end{aligned}
$$

By (5.6), $\varphi(0)$ and $\varphi(1)$ have opposite signs, proving the estimates for the eigenfunction.

We proved the estimate for the eigenfunction assuming the existence of an eigenvalue that stays bounded as $\varepsilon \rightarrow 0$. Next we prove that there is at most one eigenvalue different from $\lambda$ that stay bounded as $\varepsilon \rightarrow 0$.

Suppose that there are two eigenvalues different from $\lambda$ that stay bounded as $\varepsilon \rightarrow 0$ and let $\varphi$ and $\psi$ be the corresponding normalized eigenfunctions. Then, in addition to the above estimates, these two eigenfunctions must satisfy an orthogonality condition, which is a contradiction since

$$
\left|\int_{0}^{1} \varphi \psi d x\right|=\frac{1-x_{1}}{x_{1}} x_{1}+\frac{x_{1}}{1-x_{1}}\left(1-x_{1}\right)+O\left(\varepsilon^{\frac{1}{2}}\right)=1+O\left(\varepsilon^{\frac{1}{2}}\right) .
$$

This prove that there is at most one eigenvalue different from $\lambda$ that stay bounded as $\varepsilon \rightarrow 0$.

The next step is to prove that there is a eigenvalue different from $\lambda$ that stays bounded as $\varepsilon \rightarrow 0$. For this purpose, we exhibit a family of test functions $\psi_{\varepsilon} \in X_{\varepsilon}^{\frac{1}{2}}$, satisfying $\left\|\psi_{\varepsilon}\right\|_{L^{2}}=1$, for which the bilinear for $\left\langle A_{\varepsilon} \psi_{\varepsilon}, \psi_{\varepsilon}\right\rangle_{L^{2}}$ stays bounded as $\varepsilon \rightarrow 0$. Let $\psi_{\varepsilon}$ be such that

$$
\psi_{\varepsilon}(x)=\left\{\begin{array}{l}
-K_{1}, \quad x \in\left[0, x_{1}-\varepsilon l_{1}\right] \\
\text { linear, } \quad x \in\left[x_{1}-\varepsilon l_{1}, x_{1}+\varepsilon l_{1}\right] \\
K_{2}, \quad x \in\left[x_{1}+\varepsilon l_{1}, 1\right]
\end{array}\right.
$$

where $K_{1}, K_{2}$ are the solutions of

$$
\int_{0}^{1} \psi_{\varepsilon}(x) d x=0, \quad\left\|\psi_{\varepsilon}\right\|_{L^{2}}=1
$$

In this case

$$
K_{1}=\sqrt{\frac{1-x_{1}}{x_{1}}}+O\left(\varepsilon^{\frac{1}{2}}\right), \quad K_{2}=\sqrt{\frac{x_{1}}{1-x_{1}}}+O\left(\varepsilon^{\frac{1}{2}}\right)
$$

and

$$
\int_{0}^{1} p_{\varepsilon}(x)\left|\psi_{\varepsilon}\right|^{2} d x \leq \frac{a_{1}}{2 l_{1} x_{1}\left(1-x_{1}\right)}+\lambda+O\left(\varepsilon^{\frac{1}{2}}\right)+O\left(l_{1}-l_{1}^{\prime}\right) .
$$


Proving that $\lambda_{2}^{\varepsilon}$ stays bounded. In addition, the above inequality proves that

$$
\limsup _{\varepsilon \rightarrow 0} \lambda_{2}^{\varepsilon} \leq \frac{a_{1}}{2 l_{1} x_{1}\left(1-x_{1}\right)}+\lambda
$$

We now prove that $\lambda_{2}^{\varepsilon} \rightarrow a_{1} /\left(2 l_{1} x_{1}\left(1-x_{1}\right)\right)+\lambda$. From the estimates on the eigenfunction $\varphi_{2}$ we have

$$
\begin{aligned}
\lambda_{2}^{\varepsilon} & =\int_{0}^{1} p_{\varepsilon}\left|\varphi_{2}^{\prime}\right|^{2} d x+\int_{0}^{1} \lambda\left|\varphi_{2}\right|^{2} d x \\
& \geq \int_{x_{1}-\varepsilon l_{1}}^{x_{1}+\varepsilon l_{1}} \varepsilon a_{1}\left|\varphi_{2}^{\prime}\right|^{2} d x+\lambda \\
& \geq \frac{a_{1}}{2 l_{1}}\left(\int_{x_{1}-\varepsilon l_{1}}^{x_{1}+\varepsilon l_{1}} \varphi_{2}^{\prime} d x\right)^{2}+\lambda \\
& \geq \frac{a_{1}}{2 l_{1}}\left(\varphi_{2}\left(x_{1}+\varepsilon l_{1}\right)-\varphi_{2}\left(x_{1}-\varepsilon l_{1}\right)\right)^{2}+\lambda \\
& =\frac{a_{1}}{2 l_{1}}\left(\sqrt{\frac{1-x_{1}}{x_{1}}}+\sqrt{\frac{x_{1}}{1-x_{1}}}+O\left(\varepsilon^{\frac{1}{2}}\right)+O\left(\left(l_{1}^{\prime}-l_{1}\right)^{\frac{1}{2}}\right)\right)^{2}+\lambda \\
& =\frac{a_{1}}{2 l_{1} x_{1}\left(1-x_{1}\right)}+\lambda+O\left(\varepsilon^{\frac{1}{2}}\right)+O\left(\left(l_{1}^{\prime}-l_{1}\right)^{\frac{1}{2}}\right) .
\end{aligned}
$$

The above estimate prove that

$$
\liminf _{\varepsilon \rightarrow 0} \lambda_{2}^{\varepsilon} \geq \frac{a_{1}}{2 l_{1} x_{1}\left(1-x_{1}\right)}+\lambda
$$

The estimates (5.8) and (5.9) imply that

$$
\lim _{\varepsilon \rightarrow 0} \lambda_{2}^{\varepsilon}=\frac{a_{1}}{2 l_{1} x_{1}\left(1-x_{1}\right)}+\lambda
$$

Next, we prove that there is $\mu>0$ such that $\lambda_{3}^{\varepsilon} \geq \mu / \varepsilon$.

Let $\varphi_{3}$ be the normalized eigenfunction associated with the eigenvalue $\lambda_{3}^{\varepsilon}$ and assume that there are sequences $\varepsilon_{j} \rightarrow 0$ and $m_{j} \rightarrow 0$ as $j \rightarrow \infty$ such that

$$
\lambda_{3}^{\varepsilon_{j}} \leq \frac{m_{j}}{\varepsilon_{j}}
$$

The eigenfunction $\lambda_{3}^{\varepsilon_{j}}$ and $\varphi_{3}$ are related by

$$
\lambda_{3}^{\varepsilon_{j}}=\int_{0}^{1} p_{\varepsilon}(x)\left|\varphi_{3}^{\prime}\right|^{2} d x+\int_{0}^{1} \lambda\left|\varphi_{3}\right|^{2} d x \leq \frac{m_{j}}{\varepsilon_{j}} .
$$

Thus,

$$
\int_{0}^{x_{1}-\varepsilon l_{1}^{\prime}} \frac{e_{1}}{\varepsilon_{j}}\left|\varphi_{3}^{\prime}\right|^{2} d x \leq \int_{0}^{x_{1}-\varepsilon l_{1}^{\prime}} p_{\varepsilon}(x)\left|\varphi_{3}^{\prime}\right|^{2} d x \leq \frac{m_{j}}{\varepsilon_{j}}
$$

and

$$
\int_{0}^{x_{1}-\varepsilon l_{1}^{\prime}}\left|\varphi_{3}^{\prime}\right|^{2} d x \leq \frac{m_{j}}{e_{1}}
$$


This inequality implies that, for $x \in\left[0, x_{1}-\varepsilon_{j} l_{1}^{\prime}\right]$, we have

$$
\left|\varphi_{3}(x)-\varphi_{3}(0)\right|=\left|\int_{0}^{x} \varphi_{3}^{\prime}(s) d s\right| \leq\left(\int_{0}^{x_{1}-\varepsilon l_{1}^{\prime}}\left|\varphi_{3}^{\prime}\right|^{2} d x\right)^{\frac{1}{2}} \leq\left(\frac{m_{j}}{e_{1}}\right)^{\frac{1}{2}}
$$

which implies

$$
\varphi_{3}(x)=\varphi_{3}(0)+o(1), \quad x \in\left[0, x_{1}-\varepsilon_{j} l_{1}^{\prime}\right]
$$

Similarly

$$
\varphi_{3}(x)=\varphi_{3}(1)+o(1), \quad x \in\left[x_{1}+\varepsilon_{j} l_{1}^{\prime}, 1\right] .
$$

For the interval $\left[x_{1}-\varepsilon_{j} l_{1}^{\prime}, x_{1}-\varepsilon_{j} l_{1}\right]$, we have

$$
\int_{x_{1}-\varepsilon_{j} l_{1}^{\prime}}^{x_{1}-\varepsilon_{j} l_{1}}\left|\varphi_{3}^{\prime}\right|^{2} d x \leq \frac{m_{j}}{\varepsilon_{j}^{2} a_{1}}
$$

therefore,

$$
\begin{aligned}
\left|\varphi_{3}(x)-\varphi_{3}\left(x_{1}-\varepsilon_{j} l_{1}^{\prime}\right)\right| & =\left|\int_{x_{1}-\varepsilon_{j} l_{1}^{\prime}}^{x} \varphi_{3}^{\prime}(s) d s\right| \\
& \leq\left(\int_{x_{1}-\varepsilon_{j} l_{1}^{\prime}}^{x_{1}-\varepsilon_{j} l_{1}}\left|\varphi_{3}^{\prime}\right|^{2} d x\right)^{\frac{1}{2}}\left(\varepsilon_{j} l_{1}^{\prime}-\varepsilon_{j} l_{1}\right)^{\frac{1}{2}} \\
& \leq\left(\frac{m_{j}}{\varepsilon_{j} a_{1}}\right)^{\frac{1}{2}}\left(l_{1}^{\prime}-l_{1}\right)^{\frac{1}{2}}
\end{aligned}
$$

and

$$
\varphi_{3}(x)=\varphi_{3}\left(x_{1}-\varepsilon_{j} l_{1}^{\prime}\right)+o\left(\varepsilon_{j}^{-\frac{1}{2}}\left(l_{1}^{\prime}-l_{1}\right)^{\frac{1}{2}}\right),
$$

it follows from (5.10) that

$$
\varphi_{3}(x)=\varphi_{3}(0)+o\left(\varepsilon_{j}^{-\frac{1}{2}}\left(l_{1}^{\prime}-l_{1}\right)^{\frac{1}{2}}\right), \quad x \in\left[x_{1}-\varepsilon_{j} l_{1}^{\prime}, x_{1}-\varepsilon_{j} l_{1}\right] .
$$

In the same way

$$
\varphi_{3}(x)=\varphi_{3}(1)+o\left(\varepsilon_{j}^{-\frac{1}{2}}\left(l_{1}^{\prime}-l_{1}\right)^{\frac{1}{2}}\right), \quad x \in\left[x_{1}+\varepsilon_{j} l_{1}, x_{1}+\varepsilon_{j} l_{1}^{\prime}\right] .
$$

Finally, for $\left[x_{1}-\varepsilon_{j} l_{1}, x_{1}+\varepsilon_{j} l_{1}\right]$, we have

$$
\begin{aligned}
\left|\varphi_{3}(x)-\varphi_{3}\left(x_{1}-\varepsilon_{j} l_{1}\right)\right| & =\left|\int_{x_{1}-\varepsilon_{j} l_{1}}^{x} \varphi_{3}^{\prime}(s) d s\right| \\
& \leq\left(\int_{x_{1}-\varepsilon_{j} l_{1}}^{x_{1}+\varepsilon_{j} l_{1}}\left|\varphi_{3}^{\prime}(x)\right|^{2} d x\right)^{\frac{1}{2}}\left(2 \varepsilon_{j} l_{1}\right)^{\frac{1}{2}} \\
& \leq\left(\frac{m_{j}}{\varepsilon_{j} a_{1}}\right)^{\frac{1}{2}}\left(2 l_{1}\right)^{\frac{1}{2}}
\end{aligned}
$$

since

$$
\int_{x_{1}-\varepsilon_{j} l_{1}}^{x_{1}+\varepsilon_{j} l_{1}}\left|\varphi_{3}^{\prime}(x)\right|^{2} d x \leq \frac{m_{j}}{a_{1} \varepsilon_{j}^{2}}
$$


This implies that

$$
\left|\varphi_{3}(x)-\varphi_{3}\left(x_{1}-\varepsilon_{j} l_{1}\right)\right| \leq\left(\frac{2 m_{j} l_{1}}{\varepsilon_{j} a_{1}}\right)^{\frac{1}{2}}=o\left(\varepsilon^{-\frac{1}{2}}\right)
$$

thus,

$$
\varphi_{3}(x)=\varphi_{3}(0)+o\left(\varepsilon^{-\frac{1}{2}}\right), \quad x \in\left[x_{1}-\varepsilon_{j} l_{1}, x_{1}+\varepsilon_{j} l_{1}\right] .
$$

We now prove that $\varphi_{3}(0)$ and $\varphi_{3}(1)$ are uniformly bounded. Using estimate (5.10) and $\left\|\varphi_{3}\right\|_{L^{2}}=1$, we obtain

$$
\int_{0}^{x_{1}-\varepsilon_{j} l_{1}^{\prime}}\left(\varphi_{3}(0)+o(1)\right)^{2} \leq 1
$$

hence,

$$
0 \leq \varphi_{3}(0)^{2}+\varphi_{3}(0) o(1)+o(1) \leq \frac{1}{x_{1}-\varepsilon_{j} l_{1}^{\prime}},
$$

which proves that

$$
\varphi_{3}(0)=O(1)
$$

In a similar way we obtain

$$
\varphi_{3}(1)=O(1)
$$

In what follows we use the above estimates and the fact that $\varphi_{3}$ has average zero to obtain estimates for $\varphi_{3}(0)$ and $\varphi_{3}(1)$. In fact, we have

$$
\varphi_{3}(0) x_{1}+\varphi_{3}(1)\left(1-x_{1}\right)+o(1)=0 .
$$

Using that $\left\|\varphi_{3}\right\|_{L^{2}}=1$, we obtain

$$
\varphi_{3}(0)^{2} x_{1}+\varphi_{3}(1)^{2}\left(1-x_{1}\right)+o(1)=1 .
$$

Solving this two equations we obtain

$$
\begin{aligned}
\varphi_{3}(0)^{2} & =\frac{1-x_{1}}{x_{1}}+o(1), \\
\varphi_{3}(1)^{2} & =\frac{x_{1}}{1-x_{1}}+o(1) .
\end{aligned}
$$

In addition $\varphi_{3}(0)$ and $\varphi_{3}(1)$ have opposite sign. from the fact that $\varphi_{3}$ must be orthogonal to $\varphi_{2}$, we get a contradiction.

We now prove that the dynamic of (5.1) can be considered as a ordinary differential equation in $\mathbb{R}^{2}$.

Consider the following decomposition of $X_{\varepsilon}^{\frac{1}{2}}$. Let $Y_{\varepsilon}=\operatorname{span}\left[1, \varphi_{2}^{\varepsilon}\right]$ and $Z_{\varepsilon}=Y_{\varepsilon}^{\perp}$ its orthogonal complement in $X_{\mathcal{\varepsilon}}^{\frac{1}{2}}$. Then if $u \in X_{\mathcal{\varepsilon}}^{\frac{1}{2}}$ it can be written as

$$
u=v_{1}+v_{2} \varphi+w,
$$


where hereafter we denote $\varphi_{2}$ by $\varphi$, thus

$$
\begin{gathered}
v_{1}=\int_{0}^{1} u(x) d x, \\
v_{2}=\int_{0}^{1} u(x) \varphi(x) d x, \\
w=u-v_{1}-v_{2} \varphi .
\end{gathered}
$$

This decomposition of the space induces a decomposition in Equation (5.1) as follows. Suppose $u(t, x)$ is a solution of $(5.1)$; then,

$$
u(t, x)=v_{1}(t)+v_{2}(t) \varphi(x)+w(t, x)
$$

and

$$
\begin{gathered}
\dot{v}_{1}(t)=\int_{0}^{1} u_{t}(t, y) d y=-\lambda v_{1}(t)+\int_{0}^{1} f(u(t, y)) d y \\
\dot{v}_{2}(t)=\int_{0}^{1} u_{t}(t, y) \varphi(y) d y=-\lambda_{2} v_{2}(t)+\int_{0}^{1} f(u(t, y)) \varphi(y) d y \\
\left\{\begin{array}{l}
w_{t}=\left(p_{\varepsilon} w_{x}\right)_{x}-\lambda w+f(u(t, x))-\int_{0}^{1} f(u(t, y)) d y-\int_{0}^{1} f(u(t, y)) \varphi(y) d y \varphi(x) \\
w_{x}(0)=w_{x}(1)=0 .
\end{array}\right.
\end{gathered}
$$

We know that

$$
\varphi_{2}^{\varepsilon} \stackrel{\varepsilon \rightarrow 0}{\longrightarrow}\left\{\begin{array}{l}
-\sqrt{\frac{1-x_{1}}{x_{1}}} x \in\left(0, x_{1}\right) \\
\sqrt{\frac{x_{1}}{1-x_{1}}}, x \in\left(x_{1}, 1\right)
\end{array}\right.
$$

and $\lambda_{3}^{\varepsilon} \rightarrow \infty$ as $\varepsilon \rightarrow 0$. Therefore, one expect that the component $w$ of $u$ does not play any role in the large time behavior. Thus, the limiting equation should be

$$
\begin{aligned}
\dot{v}_{1}(t) & \approx-\lambda v_{1}(t)+\int_{0}^{1} f\left(v_{1}(t)+v_{2}(t) \varphi(y)\right) d y \\
& \approx-\lambda v_{1}(t)+x_{1} f\left(v_{1}(t)+K_{1} v_{2}(t)\right)+\left(1-x_{1}\right) f\left(v_{1}(t)+K_{2} v_{2}(t)\right), \\
\dot{v_{2}}(t) & \approx-\xi v_{2}(t)+\int_{0}^{1} f\left(v_{1}(t)+v_{2}(t) \varphi(y)\right) d y \\
& \approx-\xi v_{2}(t)-x_{1} K_{1} f\left(v_{1}(t)-K_{1} v_{2}(t)\right)+\left(1-x_{1}\right) K_{2} f\left(v_{1}(t)+K_{2} v_{2}(t)\right),
\end{aligned}
$$

where

$$
K_{1}=\sqrt{\frac{1-x_{1}}{x_{1}}}, \quad K_{2}=\sqrt{\frac{x_{1}}{1-x_{1}}} \text { and } \xi=\frac{a_{1}}{2 l_{1} x_{1}\left(1-x_{1}\right)}+\lambda .
$$

Therefore, we guess that the limiting ordinary differential equations are

$$
\left\{\begin{array}{l}
\dot{v}_{1}(t)=-\lambda v_{1}(t)+f_{1}\left(v_{1}(t), v_{2}(t)\right), \\
\dot{v}_{2}(t)=-\xi v_{2}(t)+f_{2}\left(v_{1}(t), v_{2}(t)\right)
\end{array}\right.
$$


where

$$
f_{1}\left(v_{1}(t), v_{2}(t)\right)=x_{1} f\left(v_{1}(t)+K_{1} v_{2}(t)\right)+\left(1-x_{1}\right) f\left(v_{1}(t)+K_{2} v_{2}(t)\right)
$$

and

$$
f_{2}\left(v_{1}(t), v_{2}(t)\right)=-x_{1} K_{1} f\left(v_{1}(t)-K_{1} v_{2}(t)\right)+\left(1-x_{1}\right) K_{2} f\left(v_{1}(t)+K_{2} v_{2}(t)\right)
$$

Now, consider the following change of variables,

$$
\begin{gathered}
z_{1}(t)=\frac{1}{x_{1}} \int_{0}^{x_{1}} u(t, x) d x, \\
z_{2}(t)=\frac{1}{1-x_{1}} \int_{x_{1}}^{1} u(t, x) d x
\end{gathered}
$$

where the variables in (5.11) are

$$
\begin{gathered}
v_{1}(t)=x_{1} z_{1}(t)+\left(1-x_{1}\right) z_{2}(t), \\
v_{2}(t)=-K_{1} x_{1} z_{1}(t)+K_{2}\left(1-x_{1}\right) z_{2}(t) .
\end{gathered}
$$

In the variables $z_{1}$ and $z_{2}$ we have

$$
\left[\begin{array}{c}
\dot{z}_{1} \\
\dot{z}_{2}
\end{array}\right]+\left[\begin{array}{cc}
\frac{a_{1}}{2 l_{1} x_{1}}+\lambda & -\frac{a_{1}}{2 l_{1} x_{1}} \\
-\frac{a_{1}}{2 l_{1}\left(1-x_{1}\right)} & \frac{a_{1}}{2 l_{1}\left(1-x_{1}\right)}+\lambda
\end{array}\right]\left[\begin{array}{c}
z_{1} \\
z_{2}
\end{array}\right]=\left[\begin{array}{l}
f\left(z_{1}\right) \\
f\left(z_{2}\right)
\end{array}\right] .
$$

Remark 5.1.2. From the expression of $\xi$ in the Lemma above and from the fact that $\lambda_{2}^{\varepsilon} \rightarrow \xi$ as $\varepsilon \rightarrow 0$ we observe that if one of the intervals is much smaller than the other, for $\varepsilon$ small, then there is a large gap between $\lambda$ and $\lambda_{2}^{\varepsilon}$, hence is expected that $v_{2}(t)$ will not play an important role $n$ the asymptotic behavior and in that case the limiting equations should simply be

$$
\dot{v}_{1}(t)=\lambda v_{1}(t)+f\left(v_{1}(t)\right)
$$

This is in agreement with Chapter 4, where we considered the diffusion coefficient to be large everywhere.

In what follows we denote $X_{0}$ or $X_{0}^{\frac{1}{2}}$ as $\mathbb{R}^{2}$ with the norm

$$
\left\|u^{0}\right\|_{X_{0}}^{2}=\left\langle u_{1}^{0}, u_{2}^{0}\right\rangle_{X_{0}}=\left|u_{1}^{0}\right|^{2} x_{1}+\left|u_{2}^{0}\right|^{2}\left(1-x_{1}\right)
$$

and we define the operator $A_{0}: X_{0} \rightarrow X_{0}$ given by

$$
A_{0} u^{0}=\left[\begin{array}{cc}
\frac{a_{1}}{2 l_{1} x_{1}}+\lambda & -\frac{a_{1}}{2 l_{1} x_{1}} \\
-\frac{a_{1}}{2 l_{1}\left(1-x_{1}\right)} & \frac{a_{1}}{2 l_{1}\left(1-x_{1}\right)}+\lambda
\end{array}\right]\left[\begin{array}{l}
u_{1}^{0} \\
u_{2}^{0}
\end{array}\right] .
$$

If we denote the Nemitskii functional of $f$ by the same notation $f$, then (5.1) and (5.3) can be written as

$$
\left\{\begin{array}{l}
u_{t}^{\varepsilon}+A_{\varepsilon} u^{\varepsilon}=f\left(u^{\varepsilon}\right), \\
u^{\varepsilon}(0)=u_{0}^{\varepsilon} \in X_{\mathcal{\varepsilon}}^{\frac{1}{2}}, \quad \varepsilon \in\left[0, \varepsilon_{0}\right] .
\end{array}\right.
$$


Since we have the condition (5.2) it follows from (ARRIETA; CARVALHO; RODRíGUEZBERNAL, 2000b; ARRIETA; CARVALHO; RODRíGUEZ-BERNAL, 1999; ARRIETA; CARVALHO; RODRíGUEZ-BERNAL, 2000a) and standard arguments of ordinary differential equations that we can consider $f$ bounded globally Lipschitz such that the problem (5.12), for each $\varepsilon \in\left[0, \varepsilon_{0}\right]$, is well posed for positive time and the solutions are continuously differentiable with respect to the initial data. Hence we are able to consider in $X_{\mathcal{\varepsilon}}^{\frac{1}{2}}$ the family of nonlinear semigroups $\left\{T_{\varepsilon}(\cdot)\right\}_{\varepsilon \in\left[0, \varepsilon_{0}\right]}$ defined by $T_{\varepsilon}(t)=u^{\varepsilon}\left(t, u_{0}^{\varepsilon}\right), t \geq 0$, where $u^{\varepsilon}\left(t, u_{0}^{\varepsilon}\right)$ is the solution of (5.12) through $u_{0}^{\varepsilon} \in X_{\varepsilon}^{\frac{1}{2}}$ and

$$
T_{\varepsilon}(t) u_{0}^{\varepsilon}=e^{-A_{\varepsilon} t} u_{0}^{\varepsilon}+\int_{0}^{t} e^{-A_{\varepsilon}(t-s)} f\left(T_{\varepsilon}(s)\right) d s, \quad t \geq 0,
$$

has a global attractor $\mathscr{A}_{\varepsilon}$, for each $\varepsilon \in\left(0, \varepsilon_{0}\right]$ such that $\overline{\bigcup_{\varepsilon \in\left(0, \varepsilon_{0}\right]} \mathscr{A}_{\varepsilon}}$ is compact and uniformly bounded.

We assume that (5.12) with $\varepsilon=0$ has a global attractor $\mathscr{A}_{0} \subset X_{0}^{\frac{1}{2}}$ and $T_{0}(\cdot)$ is a MorseSmale semigroup in $X_{0}^{\frac{1}{2}}$.

\subsection{Elliptic Problem}

In order to understand the problems in the same space we define the operators $E_{\varepsilon}: X_{0} \rightarrow$ $X_{\varepsilon}^{\frac{1}{2}}$ and $M_{\varepsilon}: L^{2} \rightarrow X_{0}$ given by

$$
\begin{gathered}
E_{\varepsilon}\left(u_{1}, u_{2}\right)=\left\{\begin{array}{l}
u_{1}, \quad x \in\left[0, x_{1}-\varepsilon l_{1}\right], \\
u_{1}+\frac{u_{2}-u_{1}}{\varepsilon 2 l_{1}}\left(x-x_{1}+\varepsilon l_{1}\right), x \in\left[x_{1}-\varepsilon l_{1}, x_{1}+\varepsilon l_{1}\right], \\
u_{2}, \quad x \in\left[x_{1}+\varepsilon l_{1}, 1\right],
\end{array}\right. \\
M_{\varepsilon} u=\left(u_{1}, u_{2}\right), u_{1}=\frac{1}{x_{1}-\varepsilon l_{1}} \int_{0}^{x_{1}-\varepsilon l_{1}} u d x, u_{2}=\frac{1}{1-\left(x_{1}+\varepsilon l_{1}\right)} \int_{x_{1}+\varepsilon l_{1}}^{1} u d x .
\end{gathered}
$$

It is valid the following assertions.

(i) $M_{\varepsilon} \circ E_{\varepsilon}=I_{X_{0}}$;

(ii) $\left\|E_{\varepsilon} u^{0}\right\|_{X_{\varepsilon}} \rightarrow\left\|u^{0}\right\|_{X_{0}}$ as $\varepsilon \rightarrow 0$;

(iii) $\left\|E_{\varepsilon}\right\|_{\mathscr{L}\left(X_{0}, X_{\varepsilon}\right)},\left\|M_{\mathcal{E}}\right\|_{\mathscr{L}\left(X_{\mathcal{\varepsilon}}, X_{0}\right)},\left\|E_{\mathcal{\varepsilon}}\right\|_{\mathscr{L}\left(X_{0}, X_{\varepsilon}^{\alpha}\right)},\left\|M_{\mathcal{E}}\right\|_{\mathscr{L}\left(X_{\varepsilon}^{\alpha}, X_{0}\right)} \leq C$, for some constant $C$ independent of $\varepsilon$.

Note that $E_{\varepsilon}$ is injective and $M_{\varepsilon}$ is surjective. Moreover the following inequalities are valid

$$
C^{-1}\left\|u^{0}\right\|_{X_{0}} \leq\left\|E_{\varepsilon} u^{0}\right\|_{X_{\varepsilon}^{\alpha}} \leq C\left\|u^{0}\right\|_{X_{0}}, \quad u^{0} \in X_{0} .
$$

The next lemma is essential to obtain the rate of convergence of the resolvent operators. 
Lemma 5.2.1. For $g \in L^{2}$ with $\|g\|_{L^{2}} \leq 1$ and $\varepsilon \in\left(0, \varepsilon_{0}\right]$, let $u^{\varepsilon}$ be the solution of elliptic problem $A_{\varepsilon} u^{\varepsilon}=g$ and let $u^{0}=\left(u_{1}^{0}, u_{2}^{0}\right)$ be the solution of $A_{0} u^{0}=M g$. Then there is a constant $C>0$, independent of $\varepsilon$, such that

$$
\left\|u^{\varepsilon}-E_{\varepsilon} u^{0}\right\|_{X_{\varepsilon}^{\frac{1}{2}}}^{2} \leq C\left(\left|a_{1}^{\prime}-a_{1}\right|+\left|l_{1}^{\prime}-l_{1}\right|+\varepsilon^{\frac{1}{2}}\right) .
$$

Proof. The arguments used here were inspired in (CARVALHO et al., 2010).

We denote $E=E_{\varepsilon}$. The weak solution $u^{\varepsilon}$ satisfies,

$$
\int_{0}^{1} p_{\varepsilon} u_{x}^{\varepsilon} \varphi_{x} d x+\int_{0}^{1} \lambda u^{\varepsilon} \varphi d x=\int_{0}^{1} E g u^{\varepsilon} d x, \quad \forall \varphi \in X_{\varepsilon}^{\frac{1}{2}}, \quad \varepsilon \in\left(0, \varepsilon_{0}\right]
$$

and

$\frac{a_{1}}{2 l_{1}} u_{1}^{0} \varphi_{1}^{0}+\lambda u_{1}^{0} \varphi_{1}^{0} x_{1}-\frac{a_{1}}{2 l_{1}} u_{2}^{0} \varphi_{1}^{0}-\frac{a_{1}}{2 l_{1}} u_{1}^{0} \varphi_{2}^{0}+\frac{a_{1}}{2 l_{1}} u_{2}^{0} \varphi_{1}^{2}+\lambda u_{2}^{0} \varphi_{2}^{0}\left(1-x_{1}\right)=g_{1}^{0} u_{1}^{0} x_{1}+g_{2}^{0} u_{2}^{0}\left(1-x_{1}\right)$,

for all $\varphi^{0}=\left(\varphi_{1}^{0}, \varphi_{2}^{0}\right) \in \mathbb{R}^{2}$.

We denote

$$
\begin{gathered}
\mu_{\varepsilon}=\min _{u \in X_{\varepsilon}^{\frac{1}{2}}}\left\{\frac{1}{2} \int_{0}^{1} p_{\varepsilon}\left|u_{x}\right|^{2} d x+\frac{1}{2} \int_{0}^{1} \lambda|u|^{2} d x-\int_{0}^{1} g u d x\right\} \quad \varepsilon \in\left(0, \varepsilon_{0}\right] \\
\mu_{0}=\min _{u \in \mathbb{R}^{2}}\left\{\frac{1}{2} \frac{a_{1}}{2 l_{1}}\left(u_{2}-u_{1}\right)^{2}+\frac{1}{2} \lambda\left|u_{1}\right|^{2} x_{1}+\frac{1}{2} \lambda\left|u_{2}\right|^{2}\left(1-x_{1}\right)-g_{1} u_{1} x_{1}-g_{2} u_{2}\left(1-x_{1}\right)\right\} .
\end{gathered}
$$

Then

$$
\begin{aligned}
\mu_{\varepsilon} & =\frac{1}{2} \int_{0}^{1} p_{\varepsilon}\left|u_{x}^{\varepsilon}\right|^{2} d x+\frac{1}{2} \int_{0}^{1} \lambda\left|u^{\varepsilon}\right|^{2} d x-\int_{0}^{1} g u^{\varepsilon} d x \\
& =\frac{1}{2} \int_{0}^{1} p_{\varepsilon}\left|u_{x}^{\varepsilon}-\left(E u^{0}\right)_{x}+\left(E u^{0}\right)_{x}\right|^{2} d x+\frac{1}{2} \int_{0}^{1} \lambda\left|u^{\varepsilon}-E u^{0}+E u^{0}\right|^{2} d x \\
& -\int_{0}^{1} g\left(u^{\varepsilon}-E u^{0}+E u^{0}\right) d x \\
& =\frac{1}{2} \int_{0}^{1} p_{\varepsilon}\left|u_{x}^{\varepsilon}-\left(E u^{0}\right)_{x}\right|^{2} d x+\int_{0}^{1} p_{\varepsilon}\left(u_{x}^{\varepsilon}-\left(E u^{0}\right)_{x}\right)\left(\left(E u^{0}\right)_{x}\right) d x+\frac{1}{2} \int_{0}^{1} p_{\varepsilon}\left|\left(E u^{0}\right)_{x}\right|^{2} d x \\
& +\frac{1}{2} \int_{0}^{1} \lambda\left|u^{\varepsilon}-E u^{0}\right|^{2} d x+\int_{0}^{1} \lambda\left(u^{\varepsilon}-E u^{0}\right)\left(E u^{0}\right) d x+\frac{1}{2} \int_{0}^{1} \lambda\left|E u^{0}\right|^{2} d x \\
& -\int_{0}^{1} g\left(u^{\varepsilon}-E u^{0}\right) d x-\int_{0}^{1} g E u^{0} d x
\end{aligned}
$$

We can write

$$
\int_{0}^{1} p_{\varepsilon}\left(u_{x}^{\varepsilon}-\left(E u^{0}\right)_{x}\right)\left(\left(E u^{0}\right)_{x}\right) d x=-\frac{1}{2} \int_{0}^{1} p_{\varepsilon}\left|u_{x}^{\varepsilon}-\left(E u^{0}\right)_{x}\right|^{2} d x+\frac{1}{2} \int_{0}^{1} p_{\varepsilon}\left(u_{x}^{\varepsilon}-\left(E u^{0}\right)_{x}\right) u_{x}^{\varepsilon} d x
$$




$$
\int_{0}^{1} \lambda\left(u^{\varepsilon}-E u^{0}\right)\left(E u^{0}\right) d x=-\frac{1}{2} \int_{0}^{1} \lambda\left|u^{\varepsilon}-E u^{0}\right|^{2} d x+\frac{1}{2} \int_{0}^{1} \lambda\left(u^{\varepsilon}-E u^{0}\right) u^{\varepsilon} d x .
$$

Taking $\varphi=u^{\varepsilon}-E u^{0}$ in (5.14), we have

$$
\frac{1}{2} \int_{0}^{1} p_{\varepsilon}\left(u_{x}^{\varepsilon}-\left(E u^{0}\right)_{x}\right) u_{x}^{\varepsilon} d x+\frac{1}{2} \int_{0}^{1} \lambda\left(u^{\varepsilon}-E u^{0}\right) u^{\varepsilon} d x=\int_{0}^{1} g\left(u^{\varepsilon}-E u^{0}\right) d x .
$$

Thus

$$
\mu_{\varepsilon}=-\frac{1}{2}\left\|u^{\varepsilon}-E u^{0}\right\|_{X_{\varepsilon}^{\frac{1}{2}}}^{2}+\frac{1}{2} \int_{0}^{1} p_{\varepsilon}\left|\left(E u^{0}\right)_{x}\right|^{2} d x+\frac{1}{2} \int_{0}^{1} \lambda\left|E u^{0}\right|^{2} d x-\int_{0}^{1} g E u^{0} d x .
$$

But

$$
\begin{aligned}
I_{1} & :=\frac{1}{2} \int_{0}^{1} p_{\varepsilon}\left|\left(E u^{0}\right)_{x}\right|^{2} d x+\frac{1}{2} \int_{0}^{1} \lambda\left|E u^{0}\right|^{2} d x-\int_{0}^{1} g E u^{0} d x \\
& =\frac{1}{2} \int_{x_{1}-\varepsilon l_{1}}^{x_{1}+\varepsilon l_{1}} p_{\varepsilon}\left[\frac{u_{2}^{0}-u_{1}^{0}}{\varepsilon 2 l_{1}}\right]^{2} d x+\frac{1}{2} \int_{0}^{x_{1}-\varepsilon l_{1}} \lambda\left|u_{1}^{0}\right|^{2} d x+\frac{1}{2} \int_{x_{1}-\varepsilon l_{1}}^{x_{1}+\varepsilon l_{1}} \lambda\left|E u^{0}\right|^{2} d x \\
& +\frac{1}{2} \int_{x_{1}+\varepsilon l_{1}}^{1} \lambda\left|u_{2}^{0}\right|^{2} d x-\int_{0}^{x_{1}-\varepsilon l_{1}} g_{1} u_{1}^{0} d x+\int_{0}^{x_{1}-\varepsilon l_{1}}\left(g_{1}-g\right) u_{1}^{0} d x-\int_{x_{1}-\varepsilon l_{1}}^{x_{1}+\varepsilon l_{1}} g E u^{0} d x \\
& -\int_{x_{1}+\varepsilon l_{1}}^{1} g_{2} u_{2}^{0} d x+\int_{x_{1}+\varepsilon l_{1}}^{1}\left(g_{2}-g\right) u_{2}^{0} d x
\end{aligned}
$$

and we can write

$$
\begin{aligned}
& \int_{0}^{x_{1}-\varepsilon l_{1}}\left(g_{1}-g\right) u_{1}^{0} d x=u_{1}^{0} \int_{0}^{x_{1}}\left(g_{1}-g\right) d x-u_{1}^{0} \int_{x_{1}-\varepsilon l_{1}}^{x_{1}}\left(g_{1}-g\right) d x=-\int_{x_{1}-\varepsilon l_{1}}^{x_{1}}\left(g_{1}-g\right) u_{1}^{0} d x, \\
& \int_{x_{1}+\varepsilon l_{1}}^{1}\left(g_{2}-g\right) u_{2}^{0} d x=u_{2}^{0} \int_{x_{1}}^{1}\left(g_{2}-g\right) d x-u_{2}^{0} \int_{x_{1}}^{x_{1}+\varepsilon l_{1}}\left(g_{2}-g\right) d x=-\int_{x_{1}}^{x_{1}+\varepsilon l_{1}}\left(g_{2}-g\right) u_{2}^{0} d x .
\end{aligned}
$$

Thus

$$
\begin{aligned}
I_{1} & \leq \frac{1}{2} \frac{a_{1}^{\prime}\left|u_{2}^{0}-u_{1}^{0}\right|^{2}}{\left(2 l_{1}\right)^{2} \varepsilon} \int_{x_{1}-\varepsilon l_{1}}^{x_{1}+\varepsilon l_{1}} d x+\frac{1}{2} \lambda\left|u_{1}^{0}\right|^{2}\left(x_{1}-\varepsilon l_{1}\right)+\frac{1}{2} \int_{x_{1}-\varepsilon l_{1}}^{x_{1}+\varepsilon l_{1}} \lambda\left|E u^{0}\right|^{2} d x \\
& +\frac{1}{2} \lambda\left|u_{2}^{0}\right|^{2}\left(1-x_{1}-\varepsilon l_{1}\right)-g_{1}^{0} u_{1}^{0}\left(x_{1}-\varepsilon l_{1}\right)-\int_{x_{1}-\varepsilon l_{1}}^{x_{1}+\varepsilon l_{1}} g E u^{0} d x-g_{2}^{0} u_{2}^{0}\left(1-x_{1}-\varepsilon l_{1}\right) \\
& -\int_{x_{1}-\varepsilon l_{1}}^{x_{1}}\left(g_{1}-g\right) u_{1}^{0} d x-\int_{x_{1}}^{x_{1}+\varepsilon l_{1}}\left(g_{2}-g\right) u_{2}^{0} d x \\
& \leq \mu_{0}+\frac{1}{2} \frac{\left(a_{1}-a_{1}^{\prime}\right)\left|u_{2}^{0}-u_{1}^{0}\right|^{2}}{2 l_{1}}+\frac{1}{2} \int_{x_{1}-\varepsilon l_{1}}^{x_{1}+\varepsilon l_{1}} \lambda\left|E u^{0}\right|^{2} d x-\frac{1}{2} \lambda\left|u_{1}^{0}\right|^{2} \varepsilon l_{1}-\frac{1}{2} \lambda\left|u_{2}^{0}\right|^{2} \varepsilon l_{1} \\
& +g_{1}^{0} u_{1}^{0} \varepsilon l_{1}-\int_{x_{1}-\varepsilon l_{1}}^{x_{1}+\varepsilon l_{1}} g E u^{0} d x+g_{2}^{0} u_{2}^{0} \varepsilon l_{1}-\int_{x_{1}-\varepsilon l_{1}}^{x_{1}}\left(g_{1}-g\right) u_{1}^{0} d x-\int_{x_{1}}^{x_{1}+\varepsilon l_{1}}\left(g_{2}-g\right) u_{2}^{0} d x .
\end{aligned}
$$


Therefore

$$
\frac{1}{2}\left\|u^{\varepsilon}-E u^{0}\right\|_{X_{\varepsilon}^{\frac{1}{2}}}^{2} \leq \mu_{0}-\mu_{\varepsilon}+C\left(\left|a_{1}^{\prime}-a_{1}\right|+\varepsilon^{\frac{1}{2}}\right)
$$

On the other hand,

$$
\begin{aligned}
\mu_{\varepsilon} & =\frac{1}{2} \int_{0}^{1} p_{\varepsilon}\left|u_{x}^{\varepsilon}\right|^{2} d x+\frac{1}{2} \int_{0}^{1} \lambda\left|u^{\varepsilon}\right|^{2} d x-\int_{0}^{1} g u^{\varepsilon} d x \\
& =\frac{1}{2} \int_{0}^{x_{1}-\varepsilon l_{1}^{\prime}} p_{\varepsilon}\left|u_{x}^{\varepsilon}\right|^{2} d x+\frac{1}{2} \int_{x_{1}-\varepsilon l_{1}^{\prime}}^{x_{1}+\varepsilon l_{1}^{\prime}} p_{\varepsilon}\left|u_{x}^{\varepsilon}\right|^{2} d x+\int_{x_{1}+\varepsilon l_{1}^{\prime}}^{1} p_{\varepsilon}\left|u_{x}^{\varepsilon}\right|^{2} d x \\
& +\frac{1}{2} \int_{0}^{x_{1}-\varepsilon l_{1}^{\prime}} \lambda\left|u^{\varepsilon}\right|^{2} d x+\frac{1}{2} \int_{x_{1}-\varepsilon l_{1}^{\prime}}^{x_{1}+\varepsilon l_{1}^{\prime}} \lambda\left|u^{\varepsilon}\right|^{2} d x+\frac{1}{2} \int_{x_{1}+\varepsilon l_{1}^{\prime}}^{1} \lambda\left|u^{\varepsilon}\right|^{2} d x \\
& -\int_{0}^{x_{1}-\varepsilon l_{1}^{\prime}} g u^{\varepsilon} d x-\int_{x_{1}-\varepsilon l_{1}^{\prime}}^{x_{1}+\varepsilon l_{1}^{\prime}} g u^{\varepsilon} d x-\int_{x_{1}+\varepsilon l_{1}^{\prime}}^{1} g u^{\varepsilon} d x .
\end{aligned}
$$

By Hölder's inequality we have

$$
\frac{1}{2} \frac{a_{1}}{2 l_{1}^{\prime}}\left(\int_{x_{1}-\varepsilon l_{1}^{\prime}}^{x_{1}+\varepsilon l_{1}^{\prime}} u_{x}^{\varepsilon} d x\right)^{2} \leq \frac{1}{2} \frac{a_{1}}{2 l_{1}^{\prime}} \int_{x_{1}-\varepsilon l_{1}^{\prime}}^{x_{1}+\varepsilon l_{1}^{\prime}}\left|u_{x}^{\varepsilon}\right|^{2} d x 2 \varepsilon l_{1}^{\prime} \leq \frac{1}{2} \int_{x_{1}-\varepsilon l_{1}^{\prime}}^{x_{1}+\varepsilon l_{1}^{\prime}} p_{\varepsilon}\left|u_{x}^{\varepsilon}\right|^{2} d x
$$

and we also have

$$
\begin{aligned}
& \frac{1}{2} \int_{0}^{x_{1}-\varepsilon l_{1}^{\prime}} \lambda\left|u^{\varepsilon}\right|^{2} d x \geq \int_{0}^{x_{1}-\varepsilon l_{1}^{\prime}} \lambda\left(u^{\varepsilon}-u_{1}^{\varepsilon}\right) u_{1}^{\varepsilon} d x+\frac{1}{2} \int_{0}^{x_{1}-\varepsilon l_{1}^{\prime}} \lambda\left|u_{1}^{\varepsilon}\right|^{2} d x \\
& \frac{1}{2} \int_{x_{1}+\varepsilon l_{1}^{\prime}}^{1} \lambda\left|u^{\varepsilon}\right|^{2} d x \geq \int_{x_{1}+\varepsilon l_{1}^{\prime}}^{1} \lambda\left(u^{\varepsilon}-u_{2}^{\varepsilon}\right) u_{2}^{\varepsilon} d x+\frac{1}{2} \int_{x_{1}+\varepsilon l_{1}^{\prime}}^{1} \lambda\left|u_{2}^{\varepsilon}\right|^{2} d x
\end{aligned}
$$

Thus

$$
\begin{aligned}
\mu_{\varepsilon} & \geq \frac{1}{2} \frac{a_{1}}{2 l_{1}^{\prime}}\left(\int_{x_{1}-\varepsilon l_{1}^{\prime}}^{x_{1}+\varepsilon l_{1}^{\prime}} u_{x}^{\varepsilon} d x\right)^{2}+\int_{0}^{x_{1}-\varepsilon l_{1}^{\prime}} \lambda\left(u^{\varepsilon}-u_{1}^{\varepsilon}\right) u_{1}^{\varepsilon} d x+\frac{1}{2} \int_{0}^{x_{1}-\varepsilon l_{1}^{\prime}} \lambda\left|u_{1}^{\varepsilon}\right|^{2} d x \\
& +\int_{x_{1}+\varepsilon l_{1}^{\prime}}^{1} \lambda\left(u^{\varepsilon}-u_{2}^{\varepsilon}\right) u_{2}^{\varepsilon} d x+\frac{1}{2} \int_{x_{1}+\varepsilon l_{1}^{\prime}}^{1} \lambda\left|u_{2}^{\varepsilon}\right|^{2} d x \\
& -\int_{0}^{x_{1}-\varepsilon l_{1}^{\prime}} g u^{\varepsilon} d x+\int_{0}^{x_{1}-\varepsilon l_{1}^{\prime}} g_{1} u_{1}^{\varepsilon} d x-\int_{0}^{x_{1}-\varepsilon l_{1}^{\prime}} g_{1} u_{1}^{\varepsilon} d x-\int_{x_{1}-\varepsilon l_{1}^{\prime}}^{x_{1}+\varepsilon l_{1}^{\prime}} g u^{\varepsilon} d x \\
& -\int_{x_{1}+\varepsilon l_{1}^{\prime}}^{1} g u^{\varepsilon} d x+\int_{x_{1}+\varepsilon l_{1}^{\prime}}^{1} g_{2} u_{2}^{\varepsilon} d x-\int_{x_{1}+\varepsilon l_{1}^{\prime}}^{1} g_{2} u_{2}^{\varepsilon} d x .
\end{aligned}
$$

We have 


$$
\begin{aligned}
-\int_{0}^{x_{1}-\varepsilon l_{1}^{\prime}} g u^{\varepsilon} d x & +\int_{0}^{x_{1}-\varepsilon l_{1}^{\prime}} g_{1} u_{1}^{\varepsilon} d x=\int_{0}^{x_{1}-\varepsilon l_{1}^{\prime}} g\left(u_{1}^{\varepsilon}-u^{\varepsilon}\right) d x+\int_{0}^{x_{1}-\varepsilon l_{1}^{\prime}}\left(g_{1}-g\right) u_{1}^{\varepsilon} d x \\
& =\int_{0}^{x_{1}-\varepsilon l_{1}^{\prime}} g\left(u_{1}^{\varepsilon}-u^{\varepsilon}\right) d x-\int_{x_{1}-\varepsilon l_{1}^{\prime}}^{x_{1}}\left(g_{1}-g\right) u_{1}^{\varepsilon} d x+u_{1}^{\varepsilon} \int_{0}^{x_{1}}\left(g_{1}-g\right) d x \\
& =\int_{0}^{x_{1}-\varepsilon l_{1}^{\prime}} g\left(u_{1}^{\varepsilon}-u^{\varepsilon}\right) d x-\int_{x_{1}-\varepsilon l_{1}^{\prime}}^{x_{1}}\left(g_{1}-g\right) u_{1}^{\varepsilon} d x .
\end{aligned}
$$

In the same way,

$$
-\int_{x_{1}+\varepsilon l_{1}^{\prime}}^{1} g u^{\varepsilon} d x+\int_{x_{1}+\varepsilon l_{1}^{\prime}}^{1} g_{2} u_{2}^{\varepsilon} d x=\int_{x_{1}+\varepsilon l_{1}^{\prime}}^{1} g\left(u_{2}^{\varepsilon}-u^{\varepsilon}\right) d x-\int_{x_{1}}^{x_{1}+\varepsilon l_{1}^{\prime}}\left(g_{2}-g\right) u_{2}^{\varepsilon} d x .
$$

Therefore

$$
\begin{aligned}
& \mu_{\varepsilon} \geq \frac{1}{2} \frac{a_{1}}{2 l_{1}^{\prime}}\left[u^{\varepsilon}\left(x_{1}+\varepsilon l_{1}^{\prime}\right)-u^{\varepsilon}\left(x_{1}-\varepsilon l_{1}^{\prime}\right)\right]^{2}+\frac{1}{2} \lambda\left|u_{1}^{\varepsilon}\right|^{2}\left(x_{1}-\varepsilon l_{1}^{\prime}\right)+\frac{1}{2} \lambda\left|u_{2}^{\varepsilon}\right|^{2}\left(1-x_{1}-\varepsilon l_{1}^{\prime}\right) \\
& +\int_{0}^{x_{1}-\varepsilon l_{1}^{\prime}} \lambda\left(u^{\varepsilon}-u_{1}^{\varepsilon}\right) u_{1}^{\varepsilon} d x+\int_{x_{1}+\varepsilon l_{1}^{\prime}}^{1} \lambda\left(u^{\varepsilon}-u_{2}^{\varepsilon}\right) u_{2}^{\varepsilon} d x-g_{1} u_{1}^{\varepsilon}\left(x_{1}-\varepsilon l_{1}^{\prime}\right)-g_{2} u_{2}^{\varepsilon}\left(1-x_{1}-\varepsilon l_{1}^{\prime}\right) \\
& +\int_{0}^{x_{1}-\varepsilon l_{1}^{\prime}} g\left(u_{1}^{\varepsilon}-u^{\varepsilon}\right) d x-\int_{x_{1}-\varepsilon l_{1}^{\prime}}^{x_{1}}\left(g_{1}-g\right) u_{1}^{\varepsilon} d x-\int_{x_{1}-\varepsilon l_{1}^{\prime}}^{x_{1}+\varepsilon l_{1}^{\prime}} g u^{\varepsilon} d x \\
& +\int_{x_{1}+\varepsilon l_{1}^{\prime}}^{1} g\left(u_{2}^{\varepsilon}-u^{\varepsilon}\right) d x-\int_{x_{1}}^{x_{1}+\varepsilon l_{1}^{\prime}}\left(g_{2}-g\right) u_{2}^{\varepsilon} d x \\
& =\frac{1}{2} \frac{a_{1}}{2 l_{1}}\left(u_{2}^{\varepsilon}-u_{1}^{\varepsilon}\right)^{2}+\frac{1}{2} \lambda\left|u_{1}^{\varepsilon}\right|^{2} x_{1}+\frac{1}{2} \lambda\left|u_{2}^{\varepsilon}\right|^{2}\left(1-x_{1}\right)-g_{1} u_{1}^{\varepsilon} x_{1}-g_{2} u_{2}^{\varepsilon}\left(1-x_{1}\right) \\
& -\frac{1}{2} \frac{a_{1}}{2 l_{1}}\left(u_{2}^{\varepsilon}-u_{1}^{\varepsilon}\right)^{2}+\frac{1}{2} \frac{a_{1}}{2 l_{1}^{\prime}}\left[u^{\varepsilon}\left(x_{1}+\varepsilon l_{1}^{\prime}\right)-u^{\varepsilon}\left(x_{1}-\varepsilon l_{1}^{\prime}\right)\right]^{2}-\frac{1}{2} \lambda\left|u_{1}^{\varepsilon}\right|^{2} \varepsilon l_{1}^{\prime}-\frac{1}{2} \lambda\left|u_{2}^{\varepsilon}\right|^{2} \varepsilon l_{1}^{\prime} \\
& +\int_{0}^{x_{1}-\varepsilon l_{1}^{\prime}} \lambda\left(u^{\varepsilon}-u_{1}^{\varepsilon}\right) u_{1}^{\varepsilon} d x+\int_{x_{1}+\varepsilon l_{1}^{\prime}}^{1} \lambda\left(u^{\varepsilon}-u_{2}^{\varepsilon}\right) u_{2}^{\varepsilon} d x+g_{1} u_{1}^{\varepsilon} \varepsilon l_{1}^{\prime}+g_{2} u_{2}^{\varepsilon} \varepsilon l_{1}^{\prime} \\
& +\int_{0}^{x_{1}-\varepsilon l_{1}^{\prime}} g\left(u_{1}^{\varepsilon}-u^{\varepsilon}\right) d x-\int_{x_{1}-\varepsilon l_{1}^{\prime}}^{x_{1}}\left(g_{1}-g\right) u_{1}^{\varepsilon} d x-\int_{x_{1}-\varepsilon l_{1}^{\prime}}^{x_{1}+\varepsilon l_{1}^{\prime}} g u^{\varepsilon} d x \\
& +\int_{x_{1}+\varepsilon l_{1}^{\prime}}^{1} g\left(u_{2}^{\varepsilon}-u^{\varepsilon}\right) d x-\int_{x_{1}}^{x_{1}+\varepsilon l_{1}^{\prime}}\left(g_{2}-g\right) u_{2}^{\varepsilon} d x
\end{aligned}
$$

But

$$
\begin{aligned}
\frac{1}{2} \frac{a_{1}}{2 l_{1}^{\prime}}\left[u ^ { \varepsilon } \left(x_{1}+\right.\right. & \left.\left.\varepsilon l_{1}^{\prime}\right)-u^{\varepsilon}\left(x_{1}-\varepsilon l_{1}^{\prime}\right)\right]^{2} \\
& \geq \frac{1}{2} \frac{a_{1}}{2 l_{1}^{\prime}}\left[2\left(u^{\varepsilon}\left(x_{1}+\varepsilon l_{1}^{\prime}\right)-u_{2}^{\varepsilon}+u_{1}^{\varepsilon}-u^{\varepsilon}\left(x_{1}-\varepsilon l_{1}^{\prime}\right)\right)\left(u_{2}^{\varepsilon}-u_{1}^{\varepsilon}\right)\right] \\
& +\frac{1}{2} \frac{a_{1}}{2 l_{1}^{\prime}}\left(u_{2}^{\varepsilon}-u_{1}^{\varepsilon}\right)^{2} .
\end{aligned}
$$


Thus

$$
\begin{aligned}
\mu_{0}-\mu_{\varepsilon} & \leq C\left[\left|l_{1}-l_{1}^{\prime}\right|+\varepsilon^{\frac{1}{2}}+\left|u^{\varepsilon}\left(x_{1}+\varepsilon l_{1}^{\prime}\right)-u_{2}^{\varepsilon}\right|+\left|u_{1}^{\varepsilon}-u^{\varepsilon}\left(x_{1}-\varepsilon l_{1}^{\prime}\right)\right|\right] \\
& +C\left(\left\|u_{1}^{\varepsilon}-u^{\varepsilon}\right\|_{L^{\infty}\left(0, x_{1}\right)}+\left\|u_{2}^{\varepsilon}-u^{\varepsilon}\right\|_{L^{\infty}\left(x_{1}, 1\right)}\right) .
\end{aligned}
$$

By Poincaré's inequality for average, we have

$$
\left\|u_{1}^{\varepsilon}-u^{\varepsilon}\right\|_{L^{\infty}\left(0, x_{1}\right)} \leq\left\|u_{x}^{\varepsilon}\right\|_{L^{1}\left(0, x_{1}\right)}=\int_{0}^{x_{1}}\left|u_{x}^{\varepsilon}\right| d x=\int_{0}^{x_{1}-\varepsilon l_{1}^{\prime}}\left|u_{x}^{\varepsilon}\right| d x+\int_{x_{1}-\varepsilon l_{1}^{\prime}}^{x_{1}}\left|u_{x}^{\varepsilon}\right| d x \leq C \varepsilon^{\frac{1}{2}},
$$

where we have used the Hölder's inequality and the estimate

$$
\frac{e_{1}}{\varepsilon} \int_{0}^{x_{1}-\varepsilon l_{1}^{\prime}}\left|u_{x}^{\varepsilon}\right|^{2} d x \leq \int_{0}^{x_{1}-\varepsilon l_{1}^{\prime}} p_{\varepsilon}\left|u_{x}^{\varepsilon}\right|^{2} d x \leq C
$$

In the same way, $\left\|u_{1}^{\varepsilon}-u^{\varepsilon}\right\|_{L^{\infty}\left(x_{1}, 1\right)} \leq C \varepsilon^{\frac{1}{2}}$.

Now note that

$$
\begin{aligned}
\left|u_{1}^{\varepsilon}-u^{\varepsilon}\left(x_{1}-\varepsilon l_{1}^{\prime}\right)\right| & \leq \frac{1}{x_{1}} \int_{0}^{x_{1}}\left|u^{\varepsilon}-u^{\varepsilon}\left(x_{1}-\varepsilon l_{1}^{\prime}\right)\right| d x \\
& =\frac{1}{x_{1}}\left[\int_{0}^{x_{1}-\varepsilon l_{1}^{\prime}}+\int_{x_{1}-\varepsilon l_{1}^{\prime}}^{x_{1}}\left|u^{\varepsilon}-u^{\varepsilon}\left(x_{1}-\varepsilon l_{1}^{\prime}\right)\right| d x\right] \\
& \leq \int_{0}^{x_{1}-\varepsilon l_{1}^{\prime}}\left|u^{\varepsilon}-u^{\varepsilon}\left(x_{1}-\varepsilon l_{1}^{\prime}\right)\right| d x+C \varepsilon^{\frac{1}{2}} .
\end{aligned}
$$

But for $x \in\left[0, x_{1}-\varepsilon l_{1}^{\prime}\right]$, it follows by (5.15) that

$$
\left|u^{\varepsilon}(x)-u^{\varepsilon}\left(x_{1}-\varepsilon l_{1}^{\prime}\right)\right| \leq \int_{0}^{x_{1}-\varepsilon l_{1}^{\prime}}\left|u_{x}^{\varepsilon}\right| d x \leq\left(\int_{0}^{x_{1}-\varepsilon l_{1}^{\prime}}\left|u_{x}^{\varepsilon}\right|^{2} d x\right)^{\frac{1}{2}} \leq C \varepsilon^{\frac{1}{2}} .
$$

Therefore $\left|u^{\varepsilon}(x)-u^{\varepsilon}\left(x_{1}-\varepsilon l_{1}^{\prime}\right)\right| \leq C \varepsilon^{\frac{1}{2}}$. In the same way $\left|u_{2}^{\varepsilon}-u^{\varepsilon}\left(x_{1}+\varepsilon l_{1}^{\prime}\right)\right| \leq C \varepsilon^{\frac{1}{2}}$.

Putting all estimates together we obtain (5.13).

As a consequence of the previous result, we have the following result.

Corollary 5.2.2. There is a positive constant $C$ independent of $\varepsilon$ such that

$$
\left\|A_{\mathcal{\varepsilon}}^{-1}-E_{\varepsilon} A_{0}^{-1} M_{\mathcal{\varepsilon}}\right\|_{\mathscr{L}\left(L^{2}, X_{\varepsilon}^{\frac{1}{2}}\right)}^{2} \leq C\left(\left|a_{1}^{\prime}-a_{1}\right|+\left|l_{1}^{\prime}-l_{1}\right|+\varepsilon^{\frac{1}{2}}\right) .
$$

Moreover $A_{\varepsilon}^{-1} \stackrel{\mathrm{CC}}{\longrightarrow} A_{0}^{-1}$.

Now we reassert the results of Lemmas 3.2.4 and 3.2.5.

(i) $\left\|A_{\varepsilon}^{-1}\right\|_{\mathscr{L}\left(X_{\varepsilon}^{\frac{1}{2}}\right)} \leq C$, for some constant $C$ independent of $\varepsilon$. 
(ii) Given any compact $K \subset \rho\left(A_{0}\right)$ we have for $\varepsilon$ sufficiently small (we still denote $\varepsilon \in\left(0, \varepsilon_{0}\right]$ ), $K \subset \rho\left(A_{\varepsilon}\right)$ and

$$
\sup _{\varepsilon \in\left(0, \varepsilon_{0}\right]} \sup _{\lambda \in K}\left\|\left(\lambda+A_{\varepsilon}\right)^{-1}\right\|_{\mathscr{L}\left(X_{\varepsilon}, X_{\varepsilon}^{\frac{1}{2}}\right)} \leq C,
$$

for some constant $C$ independent of $\varepsilon$. Moreover $\left(\lambda+A_{\varepsilon}\right)^{-1} \stackrel{\mathrm{CC}}{\longrightarrow}\left(\lambda+A_{0}\right)^{-1}$, for all $\lambda \in K$.

(iii) If $\mu \in \rho\left(-A_{\varepsilon}\right)$ is such that $\mu \notin\left(-\infty,-\lambda_{1}^{0}\right]$, then there is $\phi \in\left(\frac{\pi}{2}, \pi\right)$ such that $\mu \in \Sigma_{-\lambda_{1}^{0}, \phi} \backslash$ $B_{r}\left(-\lambda_{1}^{0}\right)=\left\{\mu \in \mathbb{C}\left|\arg \left(\mu+\lambda_{1}^{0}\right)\right| \leq \phi\right\} \backslash\left\{\mu \in \mathbb{C}\left|\mu+\lambda_{1}^{0}\right| \leq r\right\}$ for some small $r>0$, and it is valid the following estimate,

$$
\left\|\left(\mu+A_{\varepsilon}\right)^{-1}-E_{\varepsilon}\left(\mu+A_{0}\right)^{-1} M_{\varepsilon}\right\|_{\mathscr{L}\left(X_{\varepsilon}, X_{\varepsilon}^{\frac{1}{2}}\right)}^{2} \leq C\left(\left|a_{1}^{\prime}-a_{1}\right|+\left|l_{1}^{\prime}-l_{1}\right|+\varepsilon^{\frac{1}{2}}\right),
$$

where $C$ is a positive constant independent of $\varepsilon$ and $\mu$. Moreover, if a compact set $K \subset$ $\rho\left(A_{\varepsilon}\right) \cap \rho\left(A_{0}\right)$ then (5.16) is valid for some constant $C$ independent of $\varepsilon$ and $\mu \in K$.

\subsection{Invariant Manifold}

This section matches Section 3.3 in Chapter 3. We will estimate the convergence of invariant manifolds. This convergence can be estimated by

$$
\tau(\varepsilon)=\left(\left|a_{1}^{\prime}(\varepsilon)-a_{1}\right|+\left|l_{1}^{\prime}(\varepsilon)-l_{1}\right|+\varepsilon^{\frac{1}{2}}\right)^{\frac{1}{2}} .
$$

We have $\sigma\left(A_{0}\right)=\{\lambda, \xi\}$, where

$$
\xi=\frac{a_{1}}{2 l_{1} x_{1}\left(1-x_{1}\right)}+\lambda .
$$

Since we have the gap condition $\lambda_{j}^{\varepsilon} \rightarrow \infty$, as $\varepsilon \rightarrow 0$ for $j \geq 3$ proved in the previous section, we can take $\delta>0$ and construct, for $\varepsilon$ sufficiently small (we still denote $\varepsilon \in\left(0, \varepsilon_{0}\right]$ ), the curve $\bar{\Gamma}=\Gamma_{1}+\Gamma_{2}+\Gamma_{3}+\Gamma_{4} \subset \rho\left(-A_{\varepsilon}\right)$, where

$$
\begin{gathered}
\Gamma_{1}=\{\mu \in \mathbb{C} ; \operatorname{Re}(\mu)=-\lambda+\delta \text { and }|\operatorname{Im}(\mu)| \leq 1\}, \\
\Gamma_{2}=\{\mu \in \mathbb{C} ;-\xi-\delta \leq \operatorname{Re}(\mu) \leq-\lambda+\delta \text { and } \operatorname{Im}(\mu)=1\}, \\
\Gamma_{3}=\{\mu \in \mathbb{C} ; \operatorname{Re}(\mu)=-\xi-\delta \text { and }|\operatorname{Im}(\mu)| \leq 1\}, \\
\Gamma_{4}=\{\mu \in \mathbb{C} ;-\xi-\delta \leq \operatorname{Re}(\mu) \leq-\lambda+\delta \text { and } \operatorname{Im}(\mu)=-1\} .
\end{gathered}
$$

For $\varepsilon \in\left[0, \varepsilon_{0}\right]$ we define the spectral projection

$$
Q_{\varepsilon}=\frac{1}{2 \pi i} \int_{\bar{\Gamma}}\left(\mu+A_{\varepsilon}\right)^{-1} d \mu
$$

Note that $Q_{0}=I_{X_{0}}$ and $Q_{\varepsilon}$ is the spectral projection such that the eigenspace $Q_{\varepsilon} X_{\varepsilon}^{\frac{1}{2}}=$ $\operatorname{span}\left[\varphi_{1}^{\varepsilon}, \varphi_{2}^{\varepsilon}\right]$ is isomorphic to $\mathbb{R}^{2}$. Moreover there is a constant $C$ independent of $\varepsilon$ such that the following properties are valid, 
(i) $\left\|Q_{\varepsilon}\right\|_{\mathscr{L}\left(X_{\varepsilon}, X_{\varepsilon}^{\frac{1}{2}}\right)} \leq C$

(ii) $\left\|Q_{\varepsilon} E_{\varepsilon}-E_{\varepsilon}\right\|_{\mathscr{L}\left(X_{0} X_{\varepsilon}^{\frac{1}{2}}\right)} \leq C\left(\left|a_{1}^{\prime}-a_{1}\right|+\left|l_{1}^{\prime}-l_{1}\right|+\varepsilon^{\frac{1}{2}}\right)^{\frac{1}{2}}$.

Following the Chapter 3 we decompose $X_{\varepsilon}^{\frac{1}{2}}=Y_{\varepsilon} \oplus Z_{\varepsilon}$, where $Y_{\varepsilon}=Q_{\varepsilon} X_{\varepsilon}^{\frac{1}{2}}$ and $Z_{\varepsilon}=\left(I-Q_{\varepsilon}\right) X_{\varepsilon}^{\frac{1}{2}}$ and define the projected operators

$$
A_{\varepsilon}^{+}=\left.A_{\varepsilon}\right|_{Y_{\varepsilon}} \quad \text { and } \quad A_{\varepsilon}^{-}=\left.A_{\varepsilon}\right|_{Z_{\varepsilon}}
$$

We know that $-A_{\varepsilon}^{-}$generates a semigroup in $Z_{\varepsilon},-A_{\varepsilon}^{+}$generates a group in $Y_{\varepsilon}$ and the following estimates in the Lemma 3.2.10 are valid,

(i) $\left\|e^{-A_{\varepsilon}^{-} t} z\right\|_{X_{\varepsilon}^{\frac{1}{2}}} \leq M e^{-\beta t}\|z\|_{X_{\varepsilon}^{\frac{1}{2}}}, z \in Z_{\varepsilon}, t>0$,

(ii) $\left\|e^{-A_{\varepsilon}^{-} t} z\right\|_{X_{\varepsilon}^{\frac{1}{2}}} \leq M e^{-\beta t} t^{-\frac{1}{2}}\|z\|_{L^{2}}, z \in Z_{\varepsilon}, t>0$,

(iii) $\left\|e^{-A_{\varepsilon}^{+} t} z\right\|_{X_{\varepsilon}^{\frac{1}{2}}} \leq M e^{-\bar{\gamma} t}\|z\|_{X_{\varepsilon}^{\frac{1}{2}}}, z \in Y_{\varepsilon}, t>0$,

(iv) $\left\|e^{-A_{\varepsilon}^{+} t} z\right\|_{X_{\varepsilon}^{\frac{1}{2}}} \leq M e^{-\gamma t}\|z\|_{X_{\varepsilon}^{\frac{1}{2}}}, z \in Y_{\varepsilon}, t<0$,

(v) $\left\|E_{\varepsilon} e^{-A_{0} t} M_{\mathcal{E}} z\right\|_{X_{\varepsilon}^{\frac{1}{2}}} \leq M e^{-\gamma t}\|z\|_{X_{\varepsilon}^{\frac{1}{2}}}, z \in X_{\mathcal{\varepsilon}}^{\frac{1}{2}}, t<0$,

(vi) $\left\|e^{-A_{\varepsilon}^{+} t} z-E_{\varepsilon} e^{-A_{0} t} M_{\varepsilon} z\right\|_{X_{\varepsilon}^{\frac{1}{2}}} \leq M e^{-\gamma t}\left(\left|a_{1}^{\prime}-a_{1}\right|+\left|l_{1}^{\prime}-l_{1}\right|+\varepsilon^{\frac{1}{2}}\right)^{\frac{1}{2}}\|z\|_{X_{\varepsilon}^{\frac{1}{2}}}, t<0$,

(vii) $\left\|e^{-A_{\varepsilon}^{+} t} z-E_{\varepsilon} e^{-A_{0} t} M_{\varepsilon} z\right\|_{X_{\varepsilon}^{\frac{1}{2}}} \leq M\left(\left|a_{1}^{\prime}-a_{1}\right|+\left|l_{1}^{\prime}-l_{1}\right|+\varepsilon^{\frac{1}{2}}\right)^{\frac{1}{2}}\|z\|_{X_{\varepsilon}^{\frac{1}{2}}}, z \in Y_{\varepsilon}, t>0$,

where $\beta=\lambda_{3}^{\varepsilon} \rightarrow \infty$ as $\varepsilon \rightarrow 0, \bar{\gamma}=\lambda, \gamma=\xi+\delta$ and $M$ is a constant independent of $\varepsilon$.

Theorem 5.3.1. For $\varepsilon$ sufficiently small there is an invariant manifold $\mathscr{M}_{\varepsilon}$ for (5.12), which is given by graph of a certain Lipschitz continuous map $s_{*}^{\varepsilon}: Y_{\varepsilon} \rightarrow Z_{\varepsilon}$ as

$$
\mathscr{M}_{\varepsilon}=\left\{u^{\varepsilon} \in X_{\varepsilon}^{\frac{1}{2}} ; u^{\varepsilon}=Q_{\varepsilon} u^{\varepsilon}+s_{*}^{\varepsilon}\left(Q_{\varepsilon} u^{\varepsilon}\right)\right\} .
$$

The map $s_{*}^{\varepsilon}: Y_{\varepsilon} \rightarrow Z_{\varepsilon}$ satisfies the condition

$$
\left\|s_{*}^{\varepsilon}\right\|\left\|=\sup _{v^{\varepsilon} \in Y_{\varepsilon}}\right\| s_{*}^{\varepsilon}\left(v^{\varepsilon}\right) \|_{X_{\varepsilon}^{\frac{1}{2}}} \leq C\left(\left|a_{1}^{\prime}-a_{1}\right|+\left|l_{1}^{\prime}-l_{1}\right|+\varepsilon^{\frac{1}{2}}\right)^{\frac{1}{2}},
$$

for some positive constant $C$ independent of $\varepsilon$. The invariant manifold $\mathscr{M}_{\varepsilon}$ is exponentially attracting and the global attractor $\mathscr{A}_{\varepsilon}$ of the problem (5.12) lying in $\mathscr{M}_{\varepsilon}$ and the flow on $\mathscr{A}_{\varepsilon}$ is given by

$$
u^{\varepsilon}(t)=v^{\varepsilon}(t)+s_{*}^{\varepsilon}\left(v^{\varepsilon}(t)\right), \quad t \in \mathbb{R}
$$

where $v^{\varepsilon}(t)$ satisfy

$$
\dot{v}^{\varepsilon}+A_{\varepsilon}^{+} v^{\varepsilon}=Q_{\varepsilon} f_{\varepsilon}\left(v^{\varepsilon}+s_{*}^{\varepsilon}\left(v^{\varepsilon}(t)\right)\right)
$$

Proof. The proof is analogous to the proof of the Theorem 3.3.1. 


\subsection{Rate of Attraction}

This section matches Section 3.4 in Chapter 3. We will estimate the convergence of attractors in the Hausdorff's metric.

The invariant manifold $\mathscr{M}_{\varepsilon}, \varepsilon \in\left(0, \varepsilon_{0}\right]$, is such that $\mathscr{A}_{\varepsilon} \subset \mathscr{M}_{\varepsilon}$ and the flow is given by

$$
u^{\varepsilon}(t)=v^{\varepsilon}(t)+s_{*}^{\varepsilon}\left(v^{\varepsilon}(t)\right), \quad t \in \mathbb{R},
$$

where $v^{\varepsilon}(t)$ satisfy the following ordinary differential equation

$$
v^{\dot{\varepsilon}}+A_{\varepsilon}^{+} v^{\varepsilon}=Q_{\varepsilon} f\left(v^{\varepsilon}+s_{*}^{\varepsilon}\left(v^{\varepsilon}(t)\right)\right), \quad v^{\varepsilon} \in Y_{\varepsilon}
$$

We have

(i) $H_{\mathcal{\varepsilon}}\left(v^{\varepsilon}\right)=Q_{\varepsilon} f\left(v^{\varepsilon}+s_{*}^{\varepsilon}\left(v^{\varepsilon}(t)\right)\right)$ a continuously differentiable map in $Y_{\mathcal{\varepsilon}}$.

(ii) $\tilde{T}_{\mathcal{E}}(t)=$ the nonlinear semigroup generated by the solution of (5.17).

(iii) $\tilde{T}_{\mathcal{E}}=\tilde{T}_{\mathcal{E}}(1)$ and $T_{0}=T_{0}(1)$.

By the Chapter 3 we have the following estimates for the nonlinear semigroup reduced to the invariant manifold.

(i) For each $w^{\varepsilon} \in \mathscr{A}_{\varepsilon}$ and $w^{0} \in \mathscr{A}_{0}$, there is a positive constant $C$ independent of $\varepsilon$ such that

$$
\left\|\tilde{T}_{\varepsilon}-E_{\varepsilon} T_{0} M_{\varepsilon}\right\|_{\mathscr{L}\left(Y_{\varepsilon}, X_{\varepsilon}^{\frac{1}{2}}\right)} \leq C\left(\left|a_{1}^{\prime}-a_{1}\right|+\left|l_{1}^{\prime}-l_{1}\right|+\varepsilon^{\frac{1}{2}}\right)^{\frac{1}{2}}
$$

and

$$
\left\|E_{\varepsilon} T_{0} M_{\varepsilon} Q_{\varepsilon} w^{\varepsilon}-E_{\varepsilon} T_{0} w^{0}\right\|_{X_{\varepsilon}^{\frac{1}{2}}} \leq C\left\|Q_{\varepsilon} w^{\varepsilon}-E_{\varepsilon} w^{0}\right\|_{X_{\varepsilon}^{\frac{1}{2}}}
$$

Therefore we can reassert the Theorem 3.4.5.

Theorem 5.4.1. Let $\mathscr{A}_{\varepsilon}$ be the attractor for (5.12) and $\mathscr{A}_{0}$ the attractor of the (5.12) with $\varepsilon=0$. Then there is a positive constant $C$ independent of $\varepsilon$ such that

$$
\mathrm{d}_{\varepsilon}\left(\mathscr{A}_{\varepsilon}, E_{\varepsilon} \mathscr{A}_{0}\right) \leq C\left(\left|a_{1}^{\prime}-a_{1}\right|+\left|l_{1}^{\prime}-l_{1}\right|+\varepsilon^{\frac{1}{2}}\right)^{\frac{1}{2}}+\mathrm{d}_{\varepsilon}\left(Q_{\varepsilon} \mathscr{A}_{\varepsilon}, E_{\varepsilon} \mathscr{A}_{0}\right) .
$$

We want to estimate

$$
\mathrm{d}_{\varepsilon}\left(Q_{\varepsilon} \mathscr{A}_{\varepsilon}, E_{\varepsilon} \mathscr{A}_{0}\right) .
$$

Recall that we denoted $\left\{\varphi_{1}^{0}=1, \varphi_{2}^{0}\right\}$ the eigenfunctions associated with the two eigenvalues of $A_{0}$. Since $Y_{\varepsilon}$ and $X_{0}$ are two dimensional, we can consider

$$
Y_{\varepsilon}=\operatorname{span}\left[Q_{\varepsilon} E_{\varepsilon} 1, Q_{\varepsilon} E_{\varepsilon} \varphi_{2}^{0}\right] \quad \text { and } \quad X_{0}=\operatorname{span}\left[1, \varphi_{2}^{0}\right]
$$


and define the isomorphisms $j_{\varepsilon}: Y_{\varepsilon} \rightarrow \mathbb{R}^{2}$ and $j_{0}: X_{0} \rightarrow \mathbb{R}^{2}$ by

$$
\sum_{j=1}^{2} z_{j}^{\varepsilon} Q_{\varepsilon} E_{\varepsilon} \varphi_{j}^{0} \stackrel{j_{\varepsilon}}{\longrightarrow}\left(z_{1}^{\varepsilon}, z_{2}^{\varepsilon}\right) \quad \text { and } \quad \sum_{j=1}^{2} z_{j}^{0} \varphi_{j}^{0} \stackrel{j_{0}}{\longrightarrow}\left(z_{1}^{0}, z_{2}^{0}\right)
$$

where in $\mathbb{R}^{2}$ we consider the following norm

$$
\|z\|_{\mathbb{R}^{2}}=\left(\sum_{j=1}^{2} z_{i}^{2}\left(\lambda_{i}^{\varepsilon}\right)\right)^{\frac{1}{2}}, \quad z=\left(z_{1}, z_{2}\right) \in \mathbb{R}^{2},
$$

where $\left\{\lambda_{i}^{\varepsilon}\right\}_{j=1}^{2}$ is the first two eigenvalues of $A_{\varepsilon}$.

Now we can reassert the Lemma 3.4.3

Lemma 5.4.2. For $\bar{w}^{\varepsilon} \in Y_{\varepsilon}$ and $w^{0} \in X_{0}$ we have the following inequality

$$
\left\|j_{\varepsilon} \bar{w}^{\varepsilon}-j_{0} w^{0}\right\|_{\mathbb{R}^{2}} \leq C\left(\left\|\bar{w}^{\varepsilon}-E_{\varepsilon} w^{0}\right\|_{X_{\varepsilon}^{\alpha}}+\left(\left|a_{1}^{\prime}-a_{1}\right|+\left|l_{1}^{\prime}-l_{1}\right|+\varepsilon^{\frac{1}{2}}\right)^{\frac{1}{2}}\right),
$$

where $C$ is a constant independent of $\varepsilon$.

Following the Chapter 3, we consider the systems generated by following ordinary differential equations,

$$
\begin{aligned}
& \left\{\begin{array}{l}
\dot{v}^{\varepsilon}(t)+A_{\varepsilon}^{+} v^{\varepsilon}(t)=H_{\mathcal{\varepsilon}}\left(v^{\varepsilon}(t)\right), \quad t \in \mathbb{R}, \\
v^{\varepsilon}(0)=\bar{w}^{\varepsilon} \in Y_{\mathcal{E}}, \quad \varepsilon \in\left(0, \varepsilon_{0}\right],
\end{array}\right. \\
& \left\{\begin{array}{l}
\dot{u}^{0}(t)+A_{0} u^{0}(t)=f_{0}\left(u^{0}(t)\right), \quad t \in \mathbb{R}, \\
u^{0}(0)=M_{\mathcal{\varepsilon}} \bar{w}^{\varepsilon} \in X_{0} .
\end{array}\right.
\end{aligned}
$$

Let $z_{0}^{0}, z_{0}^{\varepsilon} \in \mathbb{R}^{2}$ such that $\bar{w}^{\varepsilon}=j_{\varepsilon}^{-1} z_{0}^{\varepsilon}$ and $M_{\mathcal{\varepsilon}} \bar{w}^{\varepsilon}=j_{0}^{-1} z_{0}^{0}$, then $j_{\varepsilon}\left(v^{\varepsilon}(t)\right)$ and $j_{0}\left(u^{0}(t)\right)$ satisfy the following equation in $\mathbb{R}^{2}$,

$$
\begin{aligned}
& \left\{\begin{array}{l}
\dot{z}^{\varepsilon}(t)+j_{\varepsilon} A_{\varepsilon}^{+} j_{\varepsilon}^{-1} z^{\varepsilon}(t)=j_{\varepsilon} H_{\varepsilon}\left(j_{\varepsilon}^{-1} z^{\varepsilon}(t)\right), \quad t \in \mathbb{R}, \\
z^{\varepsilon}(0)=z_{0}^{\varepsilon}, \quad \varepsilon \in\left(0, \varepsilon_{0}\right],
\end{array}\right. \\
& \left\{\begin{array}{l}
\dot{z}^{0}(t)+j_{0} A_{0} j_{0}^{-1} z^{0}(t)=j_{0} f_{0}\left(j_{0}^{-1} z^{0}(t)\right), \quad t \in \mathbb{R}, \\
z^{0}(0)=z_{0}^{0} .
\end{array}\right.
\end{aligned}
$$

Since we assume that the limiting problem (5.3) generates a Morse-Smale semigroup in $X_{0}$, the perturbed problem (5.1) generate a Morse-Smale semigroup in $X_{\varepsilon}^{\frac{1}{2}}$, thus if we denote $S_{0}$ and $S_{\varepsilon}$ the time one map of the systems (5.18) and (5.19), we have $S_{0}$ and $S_{\varepsilon}$ Morse-Smale semigroups and if we denote $\overline{\mathscr{A}}_{0}$ and $\overline{\mathscr{A}}_{\varepsilon}$ are its attractors respectively and we have

$$
\operatorname{dist}_{\mathbb{R}^{n}}\left(\overline{\mathscr{A}}_{\mathcal{E}}, \overline{\mathscr{A}}_{0}\right) \leq C\left\|S_{\varepsilon}-S_{0}\right\|_{L^{\infty}\left(\mathbb{R}^{2}, \mathbb{R}^{2}\right)},
$$

where $C$ is a constant independent of $\varepsilon$.

We also have the following result. 
Proposition 5.4.3. There is a constant $C$ independent of $\varepsilon$ such that

$$
\left\|S_{\varepsilon}-S_{0}\right\|_{L^{\infty}\left(\mathbb{R}^{2}, \mathbb{R}^{2}\right)} \leq C\left(\left|a_{1}^{\prime}-a_{1}\right|+\left|l_{1}^{\prime}-l_{1}\right|+\varepsilon^{\frac{1}{2}}\right)^{\frac{1}{2}} .
$$

Proof. The proof is analogous to the proof of the Theorem 3.4.4.

Finally we can estimate the continuity of attractor by rate of convergence of resolvent operators.

Theorem 5.4.4. There is a constant $C$ independent of $\varepsilon$ such that

$$
\mathrm{d}_{\mathcal{\varepsilon}}\left(Q_{\varepsilon} \mathscr{A}_{\mathcal{E}}, E_{\varepsilon} \mathscr{A}_{0}\right) \leq C\left(\operatorname{dist}_{\mathbb{R}^{2}}\left(\overline{\mathscr{A}}_{\varepsilon}, \overline{\mathscr{A}}_{0}\right)+\left(\left|a_{1}^{\prime}-a_{1}\right|+\left|l_{1}^{\prime}-l_{1}\right|+\varepsilon^{\frac{1}{2}}\right)^{\frac{1}{2}}\right) .
$$

Proof. The proof is analogous to the proof of the Theorem 3.4.5.

Theorem 5.4.5. There is a positive constant $C$ independent of $\varepsilon$ such that

$$
\mathrm{d}_{\varepsilon}\left(\mathscr{A}_{\varepsilon}, E_{\varepsilon} \mathscr{A}_{0}\right) \leq C\left(\left|a_{1}^{\prime}-a_{1}\right|+\left|l_{1}^{\prime}-l_{1}\right|+\varepsilon^{\frac{1}{2}}\right)^{\frac{1}{2}} .
$$

Proof. Since all conditions of the Chapter 3 are satisfied, the result follows by Theorem 3.4.6. 

CHAPTER

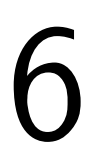

(1)

\section{SCALAR PARABOLIC EQUATIONS WITH LOCALIZED LARGE DIFFUSION}

In this chapter we study the asymptotic nonlinear dynamics of scalar semilinear parabolic problems reaction-diffusion type when the diffusion coefficient becomes large in a subregion which is interior to the domain. We obtain, under suitable assumptions, that the family of attractors behaves continuously and we exhibit the rate of convergence. An accurate description of localized large diffusion is necessary.

\subsection{Introduction}

Local spatial homogenization is a natural feature that appears in several physical phenomena, it is often present in heat conduction in composite materials for which the heat may be conducted much more quickly in some regions than other where the heat can be more slow. Reaction-diffusion problems for which the diffusivity vary considerably one region to another have solutions that tend to become spatially homogeneous in the regions where the diffusion is large. There are many results available for mathematical models with such properties (see, for example, (ARRIETA; CARVALHO; RODRíGUEZ-BERNAL, 2000b; CARBONE; CARVALHO; SCHIABEL-SILVA, 2008) and (RODRíGUEZ-BERNAL, 1998)). In (CARVALHO; PEREIRA, 1994) the authors considered a scalar parabolic problem where the diffusion coefficient is large except in a neighborhood of a finite number of point where it becomes small, see Chapter 5 and (FUSCO, 1987) where it was shown that the asymptotic behavior is described by a system of linearly coupled ordinary differential equations. The analisys in (CARVALHO; PEREIRA, 1994) requires detailed description of the transition of diffusion between the large and small parts.

In this chapter we discuss scalar parabolic problems with localized large diffusion, that is, the situation when the difusivity becomes large in a subregion which is interior of the domain. In order to obtain a rate of convergence of attractors we will make an accurate description of 
localized large diffusion.

To better present the main ideas while avoiding excessive notation, we consider the case where the diffusion is large only in a part of the domain, leaving the case where the diffusion is large in a finite number of part of the domain implicit.

Consider the scalar parabolic problem

$$
\left\{\begin{array}{l}
u_{t}^{\varepsilon}-\left(p_{\varepsilon}(x) u_{x}^{\varepsilon}\right)_{x}+(\lambda+c(x)) u^{\varepsilon}=f\left(u^{\varepsilon}\right), \quad 0<x<1, t>0 \\
u_{x}^{\varepsilon}(0)=u_{x}^{\varepsilon}(1)=0, \quad t>0 \\
u^{\varepsilon}(0)=u_{0}^{\varepsilon}
\end{array}\right.
$$

where $\varepsilon \in\left(0, \varepsilon_{0}\right]$ is a parameter $\left(0<\varepsilon_{0}<1\right), c \in C^{1}([0,1])$ is such that $c(x)=c_{\Omega_{0}}$ for all $x \in\left(x_{1}, x_{2}\right)$ where $c_{\Omega_{0}}$ is a constant, $f \in C^{2}(\mathbb{R})$ and $\lambda \in \mathbb{R}$ is such that

$$
0<m_{0} \leq \min _{x \in[0,1]} c(x)+\lambda
$$

for some positive constant $m_{0}$.

To describe the coefficients $p_{\varepsilon}$, let $0=x_{0}<x_{1}<x_{2}<x_{3}=1$ be a partition of the interval $\Omega=(0,1)$. We assume the diffusion is very large in the open interval $\Omega_{0}=\left(x_{1}, x_{2}\right)$ and converges uniformly to $p_{0} \in C^{2}\left(\Omega_{1}\right)$ in the $\Omega_{1}=\left[0, x_{1}\right] \cup\left[x_{2}, 1\right]$ as $\varepsilon$ approaches to zero. More precisely, for $\varepsilon \in\left(0, \varepsilon_{0}\right], p_{\varepsilon} \in C^{2}([0,1])$ satisfies the following conditions (see Fig 11).

$$
\left\{\begin{array}{l}
p_{\varepsilon} \stackrel{\varepsilon \rightarrow 0}{\longrightarrow} p_{0}, \quad \text { uniformly in } \Omega_{1} \\
p_{\varepsilon}(x) \geq \frac{1}{\varepsilon} \quad \text { in }\left[x_{1}+\varepsilon, x_{2}-\varepsilon\right] \\
m_{0} \leq p_{\varepsilon} \quad \text { in } \Omega \quad \text { and } \quad m_{0} \leq p_{0} \quad \text { in } \Omega_{1}
\end{array}\right.
$$

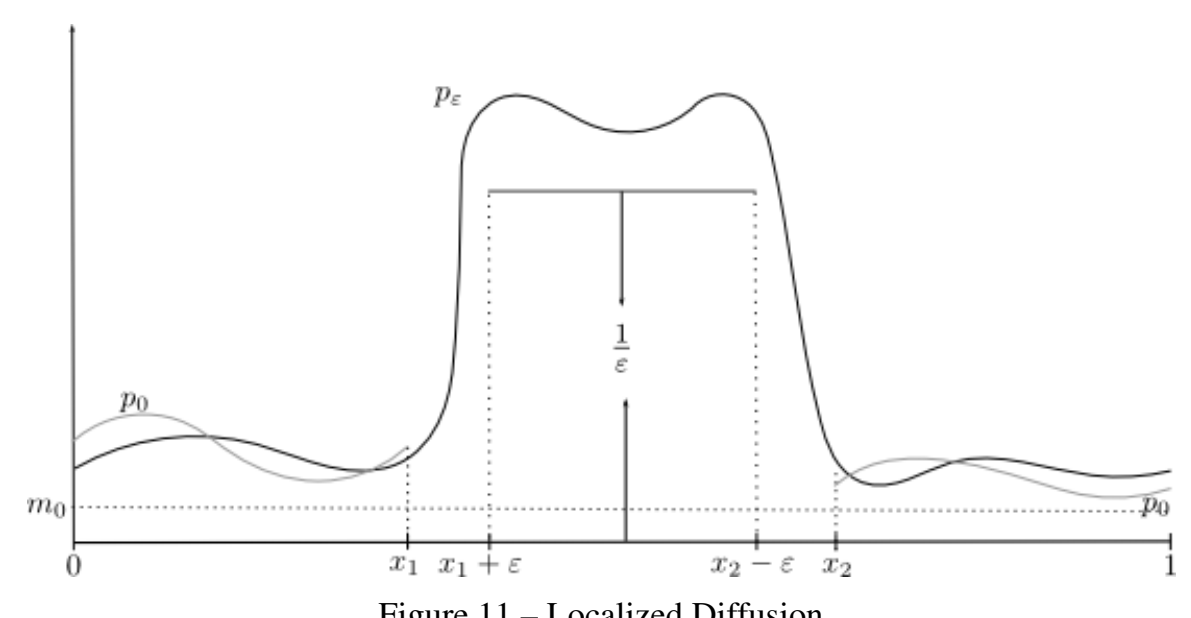

Figure 11 - Localized Diffusion

Due to the large diffusion it is natural to expect that the solutions $u^{\varepsilon}$ of the (6.1) become approximately spatially constant on $\Omega_{0}$. Thus by assuming that the solutions $u^{\varepsilon}$ exist and 
converge as $\varepsilon \rightarrow 0$, in some sense, for a function $u^{0}(t, x)$ which is spatially constant on $\left(x_{1}, x_{2}\right)$ that we denote $u^{0}(t, x)=u_{\Omega_{0}}(t)$ for all $x \in \Omega_{0}$, we expect that on $\Omega_{1}$ the solution $u^{0}$ satisfy

$$
\left\{\begin{array}{l}
u_{t}^{0}(t, x)-\operatorname{div}\left(p_{0}(x) u^{0}(t, x)\right)_{x}+(\lambda+c(x)) u^{0}(t, x)=f\left(u^{0}(t, x)\right), x \in \Omega_{1}, t>0 \\
u_{x}^{0}(0)=0=u_{x}^{0}(1)
\end{array}\right.
$$

Integrating on $\Omega_{0}$ the first equation in (6.1), we obtain

$$
\int_{\Omega_{0}} u_{t}^{\varepsilon}(t, x) d x+\int_{\Omega_{0}}\left(\lambda+c_{\Omega_{0}}\right) u^{\varepsilon}(t, x) d x=\int_{\Omega_{0}} f\left(u^{\varepsilon}(t, x)\right) d x
$$

Making $\varepsilon \rightarrow 0$ and dividing by $\left|\Omega_{0}\right|$, we have the following ordinary differential equation

$$
\dot{u}_{\Omega_{0}}^{0}+\left(\lambda+c_{\Omega_{0}}\right) u_{\Omega_{0}}^{0}=f\left(u_{\Omega_{0}}^{0}\right), x \in \Omega_{0} .
$$

We note that this equation relates the total heat flow of $\Omega_{1}$ to $\Omega_{0}$ across the boundary $\partial \Omega_{0}=$ $\left\{x_{1}, x_{2}\right\}$ with total heat in $\Omega_{1}$. Also relates the value of $u_{\Omega_{0}}^{0}$ with the value of $u^{0}$ in $\Omega_{1}$ through the integral over $\partial \Omega_{0}$.

Therefore the limiting problem of (6.1) as $\varepsilon \rightarrow 0$ is expected to be given by

$$
\left\{\begin{array}{l}
u_{t}^{0}-\left(p_{0}(x) u_{x}^{0}\right)_{x}+(\lambda+c(x)) u^{0}=f\left(u^{0}\right), \quad x \in \Omega_{1}, t>0 \\
u_{x}^{0}(0)=u_{x}^{0}(1)=0, \quad t>0 \\
\left.u^{0}\right|_{\Omega_{0}}=u_{\Omega_{0}}^{0} \\
\dot{u}_{\Omega_{0}}^{0}+\left(\lambda+c_{\Omega_{0}}\right) u_{\Omega_{0}}^{0}=f\left(u_{\Omega_{0}}^{0}\right), x \in \Omega_{0} \\
u^{0}(0)=u_{0}^{0}
\end{array}\right.
$$

where $u_{0}^{0}=\lim _{\varepsilon \rightarrow 0} u_{0}^{\varepsilon}$ is constant on $\Omega_{0}$. In (RODRíGUEZ-BERNAL, 1998) it was formally proved that (6.3) determines the asymptotic behavior of (6.1).

In order to write (6.1) and (6.3) abstractly in a suitable natural energy space we introduce some more terminology. We define the operator $A_{\varepsilon}: \mathscr{D}\left(A_{\varepsilon}\right) \subset L^{2}(0,1) \rightarrow L^{2}(0,1)$ by

$$
\mathscr{D}\left(A_{\varepsilon}\right)=\left\{u \in H^{2}(0,1) ; u_{x}(0)=u_{x}(1)=0\right\} \quad \text { and } \quad A_{\varepsilon} u=-\left(p_{\varepsilon} u_{x}\right)_{x}+(\lambda+c) u .
$$

We denote

$$
\begin{gathered}
L_{\Omega_{0}}^{2}(0,1)=\left\{u \in L^{2}(0,1) ; u \text { is constant a.e. in } \Omega_{0}\right\}, \\
H_{\Omega_{0}}^{1}(0,1)=\left\{u \in H^{1}(0,1) ; u_{x}=0 \text { in } \Omega_{0}\right\}
\end{gathered}
$$

and we define the operator $A_{0}: \mathscr{D}\left(A_{0}\right) \subset L_{\Omega_{0}}^{2}(0,1) \rightarrow L_{\Omega_{0}}^{2}(0,1)$ by

$$
\begin{gathered}
\mathscr{D}\left(A_{0}\right)=\left\{u \in H_{\Omega_{0}}^{1}(0,1) ;-\left(p_{0} u_{x}\right)_{x} \in L^{2}\left(\Omega_{1}\right), u_{x}(0)=u_{x}(1)=0\right\} ; \\
A_{0} u=\left[-\left(p_{0} u_{x}\right)_{x}+(\lambda+c) u\right] \chi_{\Omega_{1}}+\left[\left(\lambda+c_{\Omega_{0}}\right) u_{\Omega_{0}}\right] \chi_{\Omega_{0}} .
\end{gathered}
$$

It is well known (see Proposition 2.2.8) that $A_{\varepsilon}$ is a self-adjoint invertible operator with compact resolvent for each $\varepsilon \in\left[0, \varepsilon_{0}\right]$. Moreover, if $\lambda_{0}^{\varepsilon}$ is the first eigenvalue of $A_{\varepsilon}$ and $\xi>\lambda_{0}^{\varepsilon}$, then the 
operator $A_{\varepsilon}-\xi I$ is positive and hence we can define, in the usual way (see (HENRY, 1980)), the fractional power space $X_{\varepsilon}^{\frac{1}{2}}=H^{1}(0,1), \varepsilon \in\left(0, \varepsilon_{0}\right]$, and $X_{0}^{\frac{1}{2}}=H_{\Omega_{0}}^{1}(0,1)$ with the scalar products

$$
\begin{gathered}
\langle u, v\rangle_{X_{\varepsilon}^{\frac{1}{2}}}=\int_{0}^{1} p_{\varepsilon} u_{x} v_{x} d x+\int_{0}^{1}(\lambda+c) u v d x, \quad u, v \in X_{\varepsilon}^{\frac{1}{2}}, \varepsilon \in\left(0, \varepsilon_{0}\right] ; \\
\langle u, v\rangle_{X_{0}^{\frac{1}{2}}}=\int_{\Omega_{1}} p_{0} u_{x} v_{x} d x+\int_{0}^{1}(\lambda+c) u v d x, \quad u, v \in X_{0}^{\frac{1}{2}} .
\end{gathered}
$$

The space $X_{0}^{\frac{1}{2}}$ is a closed subspace of $X_{\mathcal{\varepsilon}}^{\frac{1}{2}}, \varepsilon \in\left(0, \varepsilon_{0}\right]$ and $X_{\mathcal{\varepsilon}}^{\frac{1}{2}} \subset H^{1}(0,1)$ with injection constant independent of $\varepsilon$. However a delicate issue here is that the injection $H^{1}(0,1) \subset X_{\mathcal{\varepsilon}}^{\frac{1}{2}}$ has not constant independent of $\varepsilon$, indeed is valid

$$
\|u\|_{H^{1}} \leq C\|u\|_{X_{\varepsilon}^{\frac{1}{2}}} \leq M_{\mathcal{E}}\|u\|_{H^{1}}, \quad u \in H^{1},
$$

where $M_{\varepsilon} \rightarrow \infty$ as $\varepsilon \rightarrow 0$. Actually, it can be proved that there is no possible uniform choice for $M_{\varepsilon}$ (see (CARVALHO; PIRES, 2016)), therefore estimates in the space $H^{1}$ does not guarantee estimates in $X_{\varepsilon}^{\frac{1}{2}}$. We will consider $X_{\varepsilon}^{\frac{1}{2}}$ as the phase space for the problems (6.1) and (6.3) that is, if we denote the Nemitskii functional of $f$ by the same notation $f$, then (6.1) and (6.3) can be written as

$$
\left\{\begin{array}{l}
u_{t}^{\varepsilon}+A_{\varepsilon} u^{\varepsilon}=f\left(u^{\varepsilon}\right), \\
u^{\varepsilon}(0)=u_{0}^{\varepsilon} \in X_{\mathcal{\varepsilon}}^{\frac{1}{2}}, \quad \varepsilon \in\left[0, \varepsilon_{0}\right] .
\end{array}\right.
$$

Since $X_{\mathcal{\varepsilon}}^{\frac{1}{2}} \subset C([0,1])$, we assume that the nonlinearity $f$ satisfies the following dissipativeness condition

$$
\limsup _{|x| \rightarrow \infty} \frac{f(x)}{x}<0
$$

It follows from (ARRIETA; CARVALHO; RODRíGUEZ-BERNAL, 2000b; ARRIETA; CARVALHO; RODRíGUEZ-BERNAL, 1999) and (ARRIETA; CARVALHO; RODRíGUEZBERNAL, 2000a) that we can consider $f$ bounded globally Lipschitz and the problem (6.4), for each $\varepsilon \in\left[0, \varepsilon_{0}\right]$, is well posed for positive time and the solutions are continuously differentiable with respect to the initial data. Thus we are able to consider in $X_{\varepsilon}^{\frac{1}{2}}$ the family of nonlinear semigroups $\left\{T_{\mathcal{\varepsilon}}(\cdot)\right\}_{\varepsilon \in\left[0, \varepsilon_{0}\right]}$ defined by $T_{\mathcal{\varepsilon}}(t)=u^{\varepsilon}\left(t, u_{0}^{\varepsilon}\right), t \geq 0$, where $u^{\varepsilon}\left(t, u_{0}^{\varepsilon}\right)$ is the solution of (6.4) through $u_{0}^{\varepsilon} \in X_{\mathcal{\varepsilon}}^{\frac{1}{2}}$ and

$$
T_{\mathcal{\varepsilon}}(t) u_{0}^{\varepsilon}=e^{-A_{\varepsilon} t} u_{0}^{\varepsilon}+\int_{0}^{t} e^{-A_{\varepsilon}(t-s)} f\left(T_{\mathcal{\varepsilon}}(s)\right) d s, \quad t \geq 0
$$

has a global attractor $\mathscr{A}_{\varepsilon}$, for each $\varepsilon \in\left[0, \varepsilon_{0}\right]$ such that $\overline{\bigcup_{\varepsilon \in\left[0, \varepsilon_{0}\right]} \mathscr{A}_{\varepsilon}}$ is compact and uniformly bounded.

We recall the Definition 2.3.1. A family $\left\{\mathscr{A}_{\varepsilon}\right\}_{\varepsilon \in\left[0, \varepsilon_{0}\right]}$ is continuous at $\varepsilon=0$ if

$$
\mathrm{d}_{H}\left(\mathscr{A}_{\varepsilon}, \mathscr{A}_{0}\right)=\max \left\{\operatorname{dist}_{H}\left(\mathscr{A}_{\varepsilon}, \mathscr{A}_{0}\right), \operatorname{dist}_{H}\left(\mathscr{A}_{0}, \mathscr{A}_{\varepsilon}\right)\right\} \rightarrow 0 \quad \text { as } \quad \varepsilon \rightarrow 0
$$


where

$$
\operatorname{dist}_{H}(A, B)=\sup _{a \in A} \inf _{b \in B}\|a-b\|_{X_{\varepsilon}^{\frac{1}{2}}}, \quad A, B \subset X_{\varepsilon}^{\frac{1}{2}} .
$$

We also recall that the equilibria solutions of (6.4) are those which are independent of time, that is, for $\varepsilon \in\left[0, \varepsilon_{0}\right]$, they are the solutions of the elliptic problem $A_{\varepsilon} u^{\varepsilon}-f\left(u^{\varepsilon}\right)=0$. We denote by $\mathscr{E}_{\mathcal{E}}$ the set of the equilibria solutions of $A_{\mathcal{E}}$ and we say that $u_{*}^{\varepsilon} \in \mathscr{E}_{\mathcal{E}}$ is an hyperbolic solution if $\sigma\left(A_{\varepsilon}-f^{\prime}\left(u_{*}^{\varepsilon}\right)\right) \cap\{\mu \in \mathbb{C} ; \operatorname{Re}(\mu)=0\}=\emptyset$. We assume $\mathscr{E}_{0}$ is composed of hyperbolic solutions, then $\mathscr{E}_{0}$ is finite and the family $\left\{\mathscr{E}_{\varepsilon}\right\}_{\varepsilon \in\left[0, \varepsilon_{0}\right]}$ is continuous at $\varepsilon=0$ (see (CARBONE; CARVALHO; SCHIABEL-SILVA, 2008)), thus for $\varepsilon$ sufficiently small, $\mathscr{E}_{\varepsilon}$ is composed of a finite number of hyperbolic solutions and the semigroups in (6.5) are dynamically gradient with respect to $\mathscr{E}_{\varepsilon}$, for each $\varepsilon \in\left[0, \varepsilon_{0}\right]$. Moreover in (CARBONE; RUAS-FILHO, 2006) the authors showed that the semigroup $T_{0}(\cdot)$ is Morse-Smale and the main result for Morse-Smale semigroups is stability of the phase diagram under perturbation (see (BORTOLAN et al., 2015)). In (CARBONE; RUAS-FILHO, 2006) the authors also proved the gap condition for eigenvalues of the operators $A_{\varepsilon}, \varepsilon \in\left[0, \varepsilon_{0}\right]$, and then the existence of exponential attracting finite dimensional inertial manifolds $\mathscr{M}_{\varepsilon}$ containing $\mathscr{A}_{\varepsilon}$ is ensured. Thus we can restrict the semigroups $T_{\varepsilon}$ to these inertial manifolds in order to obtain a finite dimensional problem where the robustness these inertial manifolds also ensure the geometric and topological equivalence of the attractors.

Under these assumptions, the authors in (ARRIETA; CARVALHO; RODRíGUEZBERNAL, 2000b) and (CARBONE; CARVALHO; SCHIABEL-SILVA, 2008) proved the continuity of attractors of the problem (6.4) in the phase space $X_{\varepsilon}^{\frac{1}{2}}$ however rate of attraction was not considered. The main result about rate of attraction of Morse-Smale problem is due to (SANTAMARIA; ARRIETA, 2014). They obtained an almost optimal rate of convergence of attractors involving the compact convergence of the resolvent operators $\left\|A_{\varepsilon}^{-1}-A_{0}^{-1}\right\|_{\mathscr{L}\left(L_{\Omega_{0}}^{2}, X_{\varepsilon}^{\frac{1}{2}}\right)}$. Following these ideas in this chapter we will exhibit a rate of convergence for the continuity of attractors of the problem (6.4) in the phase space $X_{\varepsilon}^{\frac{1}{2}}$ depending on the diffusion coefficients $p_{\varepsilon}$ and the parameter $\varepsilon$.

\subsection{Elliptic Problem}

In this section we analyze the solvability of the elliptic problem associated with (6.4) in order to obtain the rate of convergence of the resolvent operators. As a consequence we will estimate the convergence of the time one map $T_{\mathcal{E}}(1)$ associated with (6.5). Later we will transfer such estimate for the convergence of the time one map restricted to finite dimensional invariant manifold.

Next result establishes the convergence of the resolvent operator $\left.A_{\varepsilon}^{-1}\right|_{L_{\Omega_{0}}^{2}}$ to $A_{0}^{-1}$ and ensures that the rate of this convergence is $\left(\left\|p_{\varepsilon}-p_{0}\right\|_{L^{\infty}\left(\Omega_{1}\right)}+\varepsilon\right)^{\frac{1}{2}}$. Since we have a singular perturbation we can consider the nonlinearity on the limit space $L_{\Omega_{0}}^{2}(0,1)$. 
Lemma 6.2.1. For $g \in L_{\Omega_{0}}^{2}$ with $\|g\|_{L^{2}} \leq 1$ and $\varepsilon \in\left[0, \varepsilon_{0}\right]$, let $u^{\varepsilon}$ be the solution of elliptic problem

$$
\left\{\begin{array}{l}
A_{\varepsilon} u^{\varepsilon}=g, \quad x \in(0,1), \\
u_{x}^{\varepsilon}(0)=u_{x}^{\varepsilon}(1)=0 .
\end{array}\right.
$$

Then there is a constant $C>0$ independent of $\varepsilon$ such that

$$
\left\|u^{\varepsilon}-u^{0}\right\|_{X_{\varepsilon}^{\frac{1}{2}}} \leq C\left(\left\|p_{\varepsilon}-p_{0}\right\|_{L^{\infty}\left(\Omega_{1}\right)}+\varepsilon\right)^{\frac{1}{2}}
$$

Proof. First note that the weak solution $u^{\varepsilon}$ satisfies

$$
\begin{gathered}
\int_{0}^{1} p_{\varepsilon} u_{x}^{\varepsilon} \varphi_{x} d x+\int_{0}^{1}(\lambda+c) u^{\varepsilon} \varphi d x=\int_{0}^{1} g \varphi d x, \quad \forall \varphi \in X_{\varepsilon}^{\frac{1}{2}}, \varepsilon \in\left(0, \varepsilon_{0}\right] ; \\
\int_{\Omega_{1}} p_{0} u_{x}^{0} \varphi_{x} d x+\int_{0}^{1}(\lambda+c) u^{0} \varphi d x=\int_{0}^{1} g \varphi d x, \quad \forall \varphi \in X_{0}^{\frac{1}{2}} .
\end{gathered}
$$

If we take $\varphi=u^{\varepsilon}$ as a test function we get reach uniform bound for weak solution $u^{\varepsilon}$ in the spaces $H^{1}$ and $X_{\mathcal{\varepsilon}}^{\frac{1}{2}}$ for $\varepsilon \in\left[0, \varepsilon_{0}\right]$. Also the embedding $H^{1} \subset L^{\infty}$ gives us an uniform bound for $u^{\varepsilon}$ in the space $L^{\infty}$. Moreover we have $\left\|u_{x}^{\varepsilon}\right\|_{L^{\infty}} \leq m_{0}^{-1}\left(1+\lambda+\max _{x \in[0,1]} c(x)\right)$.

In what follows we denote by $C$ any positive constant independent of $\varepsilon$.

We define the linear operator $E: X_{\varepsilon}^{\frac{1}{2}} \rightarrow X_{0}^{\frac{1}{2}}$ by

$$
E u=\left\{\begin{array}{l}
u \text { in } \Omega_{1 \varepsilon}=\left[0, x_{1}-\varepsilon\right] \cup\left[x_{2}+\varepsilon, 1\right] \\
\text { linear in } \Omega_{\varepsilon}=\left[x_{1}-\varepsilon, x_{1}\right] \cup\left[x_{2}, x_{2}+\varepsilon\right] \\
\bar{u}:=\frac{1}{x_{2}-x_{1}} \int_{x_{1}}^{x_{2}} u d x \text { in } \Omega_{0}
\end{array}\right.
$$

for all $u \in X_{\varepsilon}^{\frac{1}{2}}$. If we let $u^{\varepsilon}-u^{0}$ as a test function in (6.6) and if we let $E\left(u^{\varepsilon}-u^{0}\right)$ as a test function in (6.7), we have

$$
\begin{aligned}
& \left\|u^{\varepsilon}-u^{0}\right\|_{X_{\varepsilon}^{\frac{1}{2}}}^{2} \leq \int_{\Omega_{\varepsilon}}|g|\left|u^{\varepsilon}-u^{0}\right| d x+\int_{\Omega_{\varepsilon}}|g|\left|E\left(u^{\varepsilon}-u^{0}\right)\right| d x+\int_{\Omega_{1 \varepsilon}}\left|p_{\varepsilon}-p_{0}\right|\left|u_{x}^{0}\right|\left|u_{x}^{\varepsilon}-u_{x}^{0}\right| d x \\
& \quad+\int_{\Omega_{\varepsilon}}\left|p_{\varepsilon}\right|\left|u_{x}^{\varepsilon}-u_{x}^{0}\right|^{2} d x+\int_{\Omega_{\varepsilon}}\left|p_{\varepsilon}\right|\left|u_{x}^{\varepsilon}\right|\left|u_{x}^{\varepsilon}-u_{x}^{0}\right| d x+\int_{\Omega_{\varepsilon}}\left|p_{0}\right|\left|u_{x}^{0}\right|\left|E\left(u^{\varepsilon}-u^{0}\right)_{x}\right| d x \\
& \quad+\int_{\Omega_{\varepsilon}}|\bar{\lambda}|\left|u^{\varepsilon}\right|\left|u^{\varepsilon}-u^{0}\right| d x+\int_{\Omega_{\varepsilon}}|\bar{\lambda}|\left|u^{0}\right|\left|E\left(u^{\varepsilon}-u^{0}\right)\right| d x+\int_{\Omega_{\varepsilon}}|\bar{\lambda}|\left|u^{\varepsilon}-u^{0}\right|^{2} d x,
\end{aligned}
$$

where $\bar{\lambda}=\lambda+c$. Since $p_{\varepsilon}$ converges uniformly to $p_{0}$ in $\Omega_{1}$, we have $p_{\varepsilon}$ uniformly bounded in $\Omega_{1}$. Thus by Hölder inequality, (6.2) and the uniform bound for weak solution $u^{\varepsilon}$ and $u_{x}^{\varepsilon}$, the right hand side of the above expression can be estimated by

$$
C\left\|u^{\varepsilon}-u^{0}\right\|_{X_{\varepsilon}^{\frac{1}{2}}}\left(\left\|p_{\varepsilon}-p_{0}\right\|_{L^{\infty}\left(\Omega_{1}\right)}+\varepsilon^{\frac{1}{2}}\right) .
$$


For terms that evolve the operator $E$ we have used

$$
\int_{\Omega_{\varepsilon}}\left|E\left(u^{\varepsilon}-u^{0}\right)_{x}\right| d x \leq C\left\|u^{\varepsilon}-u^{0}\right\|_{X_{\varepsilon}^{\frac{1}{2}}} \varepsilon^{\frac{1}{2}} \text { and }\left\|E\left(u^{\varepsilon}-u^{0}\right)\right\|_{L^{\infty}\left(\Omega_{\varepsilon}\right)} \leq C\left\|u^{\varepsilon}-u^{0}\right\|_{X_{\varepsilon}^{\frac{1}{2}}} .
$$

We will prove the first one. For this we denote $v^{\varepsilon}=u^{\varepsilon}-u^{0}$ and, just to short, we assume $\Omega_{\varepsilon}=\left[x_{1}-\varepsilon, x_{1}\right]$. In this case we have

$$
\left|E\left(v^{\varepsilon}\right)_{x}\right|=\left|\frac{\bar{v}^{\bar{\varepsilon}}-v^{\varepsilon}\left(x_{1}-\varepsilon\right)}{\varepsilon}\right| \leq\left|\frac{\bar{v}^{\varepsilon}-v^{\varepsilon}\left(x_{1}+\varepsilon\right)}{\varepsilon}\right|+\left|\frac{v^{\varepsilon}\left(x_{1}+\varepsilon\right)-v^{\varepsilon}\left(x_{1}-\varepsilon\right)}{\varepsilon}\right|,
$$

thus

$$
\begin{aligned}
\left|\bar{v}^{\varepsilon}-v^{\varepsilon}\left(x_{1}+\varepsilon\right)\right| & \leq \frac{1}{x_{2}-x_{1}} \int_{x_{1}}^{x_{2}}\left|v^{\varepsilon}-v^{\varepsilon}\left(x_{1}+\varepsilon\right)\right| d x \\
& =\frac{1}{x_{2}-x_{1}}\left[\int_{x_{1}}^{x_{1}+\varepsilon}+\int_{x_{1}+\varepsilon}^{x_{2}-\varepsilon}+\int_{x_{2}-\varepsilon}^{x_{2}}\left|v^{\varepsilon}-v^{\varepsilon}\left(x_{1}+\varepsilon\right)\right| d x\right]
\end{aligned}
$$

We have

$$
\int_{x_{1}}^{x_{1}+\varepsilon}+\int_{x_{2}-\varepsilon}^{x_{2}}\left|v^{\varepsilon}-v^{\varepsilon}\left(x_{1}+\varepsilon\right)\right| d x \leq C\left\|v^{\varepsilon}\right\|_{L^{\infty}} \varepsilon \leq C\left\|v^{\varepsilon}\right\|_{X_{\varepsilon}^{\frac{1}{2}}} \varepsilon^{\frac{1}{2}}
$$

and for $x \in\left[x_{1}+\varepsilon, x_{2}-\varepsilon\right]$,

$$
\left|v^{\varepsilon}(x)-v^{\varepsilon}\left(x_{1}+\varepsilon\right)\right| \leq \int_{x_{1}+\varepsilon}^{x_{2}-\varepsilon}\left|v_{x}^{\varepsilon}\right| d x \leq\left(\int_{x_{1}+\varepsilon}^{x_{2}-\varepsilon}\left|v_{x}^{\varepsilon}\right|^{2} d x\right)^{\frac{1}{2}}
$$

but

$$
\frac{1}{\varepsilon} \int_{x_{1}+\varepsilon}^{x_{2}-\varepsilon}\left|v_{x}^{\varepsilon}\right|^{2} d x \leq \int_{x_{1}+\varepsilon}^{x_{2}-\varepsilon} p_{\varepsilon}\left|v_{x}^{\varepsilon}\right|^{2} d x \leq\left\|v^{\varepsilon}\right\|_{X_{\varepsilon}^{\frac{1}{2}}}^{2}
$$

and then

$$
\int_{x_{1}+\varepsilon}^{x_{2}-\varepsilon}\left|v^{\varepsilon}(x)-v^{\varepsilon}\left(x_{1}+\varepsilon\right)\right| d x \leq C\left\|v^{\varepsilon}\right\|_{X_{\varepsilon}^{\frac{1}{2}}} \varepsilon^{\frac{1}{2}}
$$

We also have

$$
\left|v^{\varepsilon}\left(x_{1}+\varepsilon\right)-v^{\varepsilon}\left(x_{1}-\varepsilon\right)\right| \leq \int_{x_{1}-\varepsilon}^{x_{1}+\varepsilon}\left|v_{x}^{\varepsilon}\right| d x \leq\left(\int_{x_{1}-\varepsilon}^{x_{1}+\varepsilon}\left|v_{x}^{\varepsilon}\right|^{2} d x\right)^{\frac{1}{2}}(2 \varepsilon)^{\frac{1}{2}} \leq C\left\|v^{\varepsilon}\right\|_{X_{\varepsilon}^{\frac{1}{2}}} \varepsilon^{\frac{1}{2}} .
$$

As a consequence of the previous lemma, we have the following result.

Corollary 6.2.2. There is a positive constant $C$ independent of $\varepsilon$ such that

$$
\left\|A_{\varepsilon}^{-1}-A_{0}^{-1}\right\|_{\mathscr{L}\left(L_{\Omega_{0}}^{2}, X_{\varepsilon}^{\frac{1}{2}}\right)} \leq C\left(\left\|p_{\varepsilon}-p_{0}\right\|_{L^{\infty}\left(\Omega_{1}\right)}+\varepsilon\right)^{\frac{1}{2}} .
$$

Furthermore, there is $\phi \in\left(\frac{\pi}{2}, \pi\right)$ such that for all $\mu \in \Sigma_{\bar{\lambda}, \phi}=\{\mu \in \mathbb{C}:|\arg (\mu+\bar{\lambda})| \leq \phi\} \backslash\{\mu \in$ $\mathbb{C}:|\mu+\bar{\lambda}| \leq r\}$, where $\bar{\lambda}=\lambda+c_{\Omega_{0}}$ and $r>0$,

$$
\left\|\left(\mu+A_{\varepsilon}\right)^{-1}-\left(\mu+A_{0}\right)^{-1}\right\|_{\mathscr{L}\left(L_{\Omega_{0}}^{2}, X_{\varepsilon}^{\frac{1}{2}}\right)} \leq C\left(\left\|p_{\varepsilon}-p_{0}\right\|_{L^{\infty}\left(\Omega_{1}\right)}+\varepsilon\right)^{\frac{1}{2}} .
$$


Proof. The first part is an immediate consequence of Lemma 6.2.1. Let $\rho\left(A_{\varepsilon}\right)$ be the resolvent set of the operator $A_{\varepsilon}, \varepsilon \in\left[0, \varepsilon_{0}\right]$. If $\mu \in \rho\left(-A_{\varepsilon}\right) \cap \rho\left(-A_{0}\right)$, we choose $\varphi \in\left(\frac{\pi}{2}, \pi\right)$ suitable in order to get the sectorial estimates

$$
\left\|\left(\mu+A_{\varepsilon}\right)^{-1}\right\|_{\mathscr{L}\left(L^{2}\right)} \leq \frac{M_{\varphi}}{|\mu|}, \quad \varepsilon \in\left(0, \varepsilon_{0}\right], \quad \text { and } \quad\left\|\left(\mu+A_{0}\right)^{-1}\right\|_{\mathscr{L}\left(L_{\Omega_{0}}^{2}\right)} \leq \frac{M_{\varphi}}{|\mu|} .
$$

Therefore, $\left\|A_{\varepsilon}\left(\mu+A_{\varepsilon}\right)^{-1}\right\|_{\mathscr{L}\left(L^{2}\right)} \leq 1+M_{\varphi}$ for $\varepsilon \in\left(0, \varepsilon_{0}\right]$ and $\left\|A_{0}\left(\mu+A_{0}\right)^{-1}\right\|_{\mathscr{L}\left(L_{\Omega_{0}}^{2}\right)} \leq 1+M_{\varphi}$. But if $g \in L_{\Omega_{0}}^{2}$, we can write

$$
A_{\varepsilon}^{\frac{1}{2}}\left(\left(\mu+A_{\varepsilon}\right)^{-1}-\left(\mu+A_{0}\right)^{-1}\right) g=A_{\varepsilon}\left(\mu+A_{\varepsilon}\right)^{-1} A_{\varepsilon}^{\frac{1}{2}}\left(A_{\varepsilon}^{-1}-A_{0}^{-1}\right) A_{0}\left(\mu+A_{0}\right)^{-1} g
$$

and thus

$$
\begin{aligned}
\|\left(\mu+A_{\varepsilon}\right)^{-1}- & \left(\mu+A_{0}\right)^{-1} \|_{\mathscr{L}\left(L_{\Omega_{0}}^{2}, X_{\varepsilon}^{\frac{1}{2}}\right)} \\
& \leq\left\|A_{\mathcal{\varepsilon}}\left(\mu+A_{\mathcal{\varepsilon}}\right)^{-1}\right\|_{\mathscr{L}\left(L_{\Omega_{0}}^{2}\right)}\left\|A_{\varepsilon}^{\frac{1}{2}}\left(A_{\varepsilon}^{-1}-A_{0}^{-1}\right)\right\|_{\mathscr{L}\left(L_{\Omega_{0}}^{2}\right)}\left\|A_{0}\left(\mu+A_{0}\right)^{-1}\right\|_{\mathscr{L}\left(L_{\Omega_{0}}^{2}\right)} \\
& \leq\left\|A_{\mathcal{\varepsilon}}\left(\mu+A_{\mathcal{\varepsilon}}\right)^{-1}\right\|_{\mathscr{L}\left(L^{2}\right)}\left\|A_{\mathcal{\varepsilon}}^{\frac{1}{2}}\left(A_{\varepsilon}^{-1}-A_{0}^{-1}\right)\right\|_{\mathscr{L}\left(L_{\Omega_{0}}^{2}\right)}\left\|A_{0}\left(\mu+A_{0}\right)^{-1}\right\|_{\mathscr{L}\left(L_{\Omega_{0}}^{2}\right)} \\
& \leq C\left(\left\|p_{\varepsilon}-p_{0}\right\|_{L^{\infty}\left(\Omega_{1}\right)}+\varepsilon\right)^{\frac{1}{2}}
\end{aligned}
$$

for some constant $C=C(\varphi)>0$ independent of $\mu$ and $\varepsilon$.

In the next theorem we will obtain the rate of convergence of nonlinear semigroup. We will follow (SANTAMARIA; ARRIETA, 2014) that improves (for the Morse-Smale case) the results presented by (ARRIETA; BEZERRA; CARVALHO, 2013). For our purposes we just need to consider the time $t=1$.

Theorem 6.2.3. For each $w_{0} \in \mathscr{A}_{0}$, there is a positive constant $C$ independent of $\varepsilon$ such that

$$
\left\|T_{\mathcal{\varepsilon}}(1) w_{0}-T_{0}(1) w_{0}\right\|_{X_{\mathcal{\varepsilon}}^{\frac{1}{2}}} \leq C\left(\left\|p_{\varepsilon}-p_{0}\right\|_{L^{\infty}\left(\Omega_{1}\right)}+\varepsilon\right)^{\frac{1}{2}}\left|\log \left(\left\|p_{\varepsilon}-p_{0}\right\|_{L^{\infty}\left(\Omega_{1}\right)}+\varepsilon\right)\right| .
$$

Proof. For each $\varepsilon \in\left[0, \varepsilon_{0}\right]$ the operator $A_{\varepsilon}$ generates an analytic semigroup $\left\{e^{-A_{\varepsilon} t} ; t \geq 0\right\}$ which is given by

$$
e^{-A_{\varepsilon} t}=\frac{1}{2 \pi i} \int_{\Gamma} e^{\mu t}\left(\mu+A_{\varepsilon}\right)^{-1} d \mu
$$

where $\Gamma$ is the boundary of $\Sigma_{-\alpha, v} \backslash\{\mu \in \mathbb{C} ;|\mu+\alpha| \leq r\}$ for some small $r, v \in\left(\phi, \frac{\pi}{2}\right)$ and $\alpha>0$, oriented towards increasing of the imaginary part with $\Sigma_{-\alpha, v}=\{\mu \in \mathbb{C} ;|\arg (\mu+\alpha)| \leq v\}$. It follows that there is $0<\gamma=\gamma(\varepsilon) \rightarrow \infty$ as $\varepsilon \rightarrow 0$ such that,

$$
\begin{gathered}
\left\|e^{-A_{\varepsilon} t}\right\|_{\mathscr{L}\left(L^{2}, X_{\varepsilon}^{\frac{1}{2}}\right)} \leq M t^{-\frac{1}{2}} e^{-\gamma t}, \quad t>0, \quad \varepsilon \in\left(0, \varepsilon_{0}\right] ; \\
\left\|e^{-A_{0} t}\right\|_{\mathscr{L}\left(L_{\Omega_{0}}^{2}, X_{\varepsilon}^{\frac{1}{2}}\right)} \leq M t^{-\frac{1}{2}} e^{-\gamma t}, \quad t>0,
\end{gathered}
$$


where $M$ is a positive constant independent of $\varepsilon$. Thus for $t>0$,

$$
\begin{aligned}
\left\|e^{-A_{\varepsilon} t}-e^{-A_{0} t}\right\|_{\mathscr{L}\left(L_{\Omega_{0}}^{2}, X_{\varepsilon}^{\frac{1}{2}}\right)} & \leq\left\|e^{-A_{\varepsilon} t}\right\|_{\mathscr{L}\left(L_{\Omega_{0}}^{2}, X_{\varepsilon}^{\frac{1}{2}}\right)}+\left\|e^{-A_{0} t}\right\|_{\mathscr{L}\left(L_{\Omega_{0}}^{2}, X_{\varepsilon}^{\frac{1}{2}}\right)} \\
& \leq M t^{-\frac{1}{2}} e^{-\gamma t}+M t^{-\frac{1}{2}} e^{-\gamma t} \\
& \leq 2 M t^{-\frac{1}{2}}
\end{aligned}
$$

Moreover, using the Corollary 6.2.2, we have

$$
\begin{aligned}
\left\|e^{-A_{\varepsilon} t}-e^{-A_{0} t}\right\|_{\mathscr{L}\left(L_{\Omega_{0}}^{2}, X_{\varepsilon}^{\frac{1}{2}}\right)} & \leq \frac{1}{2 \pi} \int_{\Gamma}\left|e^{\mu t}\right|\left\|\left(\mu+A_{\varepsilon}\right)^{-1}-\left(\mu+A_{0}\right)^{-1}\right\|_{\mathscr{L}\left(L_{\Omega_{0}}^{2}, X_{\varepsilon}^{\frac{1}{2}}\right)}|d \mu| \\
& \leq C\left(\left\|p_{\varepsilon}-p_{0}\right\|_{L^{\infty}\left(\Omega_{1}\right)}+\varepsilon\right)^{\frac{1}{2}} t^{-1} .
\end{aligned}
$$

We denote $l_{\varepsilon}(t)=\max \left\{t^{-\frac{1}{2}},\left(\left\|p_{\varepsilon}-p_{0}\right\|_{L^{\infty}\left(\Omega_{1}\right)}+\varepsilon\right)^{\frac{1}{2}} t^{-1}\right\}$. Since the nonlinear semigroup is given by (6.5), then for $0<t \leq 1$, we have

$$
\begin{aligned}
\left\|T_{\varepsilon}(t) w_{0}-T_{0}(t) w_{0}\right\|_{X_{\varepsilon}^{\frac{1}{2}}} \leq\left\|\left(e^{-A_{\varepsilon} t}-e^{-A_{0} t}\right) w_{0}\right\|_{X_{\varepsilon}^{\frac{1}{2}}} & \\
& +\int_{0}^{t}\left\|e^{-A_{\varepsilon}(t-s)} f\left(T_{\varepsilon}(s) w_{0}\right)-e^{-A_{0}(t-s)} f\left(T_{0}(s) w_{0}\right)\right\|_{X_{\varepsilon}^{\frac{1}{2}}} d s,
\end{aligned}
$$

but

$$
\begin{aligned}
\int_{0}^{t} \| e^{-A_{\varepsilon}(t-s)} f\left(T_{\mathcal{E}}(s) w_{0}\right)- & e^{-A_{0}(t-s)} f\left(T_{0}(s) w_{0}\right) \|_{X_{\varepsilon}^{\frac{1}{2}}} d s \\
& \leq \int_{0}^{t}\left\|e^{-A_{\mathcal{E}}(t-s)}\left[f\left(T_{\mathcal{\varepsilon}}(s) w_{0}\right)-f\left(T_{0}(s) w_{0}\right)\right]\right\|_{X_{\varepsilon}^{\frac{1}{2}}} d s \\
& +\int_{0}^{t}\left\|e^{-A_{\varepsilon}(t-s)} f\left(T_{0}(s) w_{0}\right)-e^{-A_{0}(t-s)} f\left(T_{0}(s) w_{0}\right)\right\|_{X_{\varepsilon}^{\frac{1}{2}}} d s \\
& \left.\leq C \int_{0}^{t}(t-s)^{-\frac{1}{2}} \| T_{\varepsilon}(s) w_{0}-T_{0}(s) w_{0}\right] \|_{X_{\varepsilon}^{\frac{1}{2}}} d s \\
& +C \int_{0}^{t} l_{\varepsilon}(t-s)\left\|f\left(T_{0}(s) w_{0}\right)\right\|_{X_{\varepsilon}^{\frac{1}{2}}} d s
\end{aligned}
$$

If we used the the Lemma 3.10 in (SANTAMARIA; ARRIETA, 2014) the result follows by Gronwall's inequality (see (HENRY, 1980)) and taking $t=1$.

\subsection{Rate of Convergence of Eigenvalues and Equilibria}

In this section we will obtain the rate of convergence of eigenvalues, spectral projection and equilibrium points.

The convergence of eigenvalues and eigenfunctions of the linear operators was proved in (RODRíGUEZ-BERNAL, 1998) and the properties about the compact convergence of the spectral projections was studied in details in several works (see, for example, (CARBONE; CARVALHO; 
SCHIABEL-SILVA, 2008; CARVALHO; PISKAREV, 2006) and (RODRíGUEZ-BERNAL, 1998)).

In the next result we will follow (ARRIETA; BEZERRA; CARVALHO, 2013), where it was considered rate of convergence for the eigenvalues and spectral projections. We also state the gap condition proved in (CARBONE; RUAS-FILHO, 2006).

Lemma 6.3.1. If $\lambda^{\varepsilon} \in \sigma\left(A_{\varepsilon}\right), \varepsilon \in\left[0, \varepsilon_{0}\right]$, and $\lambda^{\varepsilon} \stackrel{\varepsilon \rightarrow 0}{\longrightarrow} \lambda^{0}$, then

$$
\left|\lambda^{\varepsilon}-\lambda^{0}\right| \leq C\left(\left\|p_{\varepsilon}-p_{0}\right\|_{L^{\infty}\left(\Omega_{1}\right)}+\varepsilon\right)^{\frac{1}{2}} .
$$

Moreover, if we denote $\sigma\left(A_{\varepsilon}\right)=\left\{\lambda_{i}^{\varepsilon}\right\}_{i=0}^{\infty}$ (ordered and counting multiplicity), we have the following gap condition

$$
\lambda_{i+1}^{\varepsilon}-\lambda_{i}^{\varepsilon} \stackrel{i \rightarrow \infty}{\longrightarrow} \infty
$$

Proof. Let $\lambda^{0} \in \sigma\left(A_{0}\right)$ be an isolated eigenvalue. We consider an appropriated closed curve $\Gamma$ in $\rho\left(-A_{0}\right)$ around $\lambda^{0}$ and define the spectral projection

$$
Q_{\varepsilon}=\frac{1}{2 \pi i} \int_{\Gamma}\left(\mu+A_{\varepsilon}\right)^{-1} d \mu, \quad \varepsilon \in\left[0, \varepsilon_{0}\right]
$$

It follows from Corollary 6.2.2 that

$$
\left\|Q_{\varepsilon}-Q_{0}\right\|_{\mathscr{L}\left(L_{\Omega_{0}}^{2}, X_{\varepsilon}^{\frac{1}{2}}\right)} \leq C\left(\left\|p_{\varepsilon}-p_{0}\right\|_{L^{\infty}\left(\Omega_{1}\right)}+\varepsilon\right)^{\frac{1}{2}}
$$

If we have $\lambda^{\varepsilon} \in \sigma\left(A_{\varepsilon}\right)$ such that $\lambda^{\varepsilon} \stackrel{\varepsilon \rightarrow 0}{\longrightarrow} \lambda^{0}$ then for $\varepsilon$ sufficiently small there is $u^{0} \in \operatorname{Ker}\left(\lambda_{0}-A_{0}\right)$ with $\left\|u^{0}\right\|_{X_{\varepsilon}^{\frac{1}{2}}}=1$ such that $Q_{\varepsilon} u^{0}$ is eigenvalue of $A_{\varepsilon}$ associated with $\lambda^{\varepsilon}$, thus

$$
\left|\lambda^{\varepsilon}-\lambda^{0}\right| \leq\left\|\left(\lambda^{\varepsilon}-\lambda^{0}\right) u^{0}\right\|_{X_{\varepsilon}^{\frac{1}{2}}}=\left\|\lambda^{\varepsilon} Q_{0} u^{0}-\lambda^{0} u^{0}\right\|_{X_{\varepsilon}^{\frac{1}{2}}}
$$

but

$$
\begin{aligned}
\left\|\lambda^{\varepsilon} Q_{0} u^{0}-\lambda^{0} u^{0}\right\|_{X_{\varepsilon}^{\frac{1}{2}}} & \leq\left\|\lambda^{\varepsilon} Q_{0} u^{0}-\lambda^{0} Q_{\varepsilon} u^{0}+\lambda^{0} Q_{\varepsilon} u^{0}-\lambda^{0} u^{0}\right\|_{X_{\varepsilon}^{\frac{1}{2}}} \\
& \leq\left|\lambda^{\varepsilon} \lambda^{0}\right|\left\|\frac{1}{\lambda^{0}} Q_{0} u^{0}-\frac{1}{\lambda^{\varepsilon}} Q_{\varepsilon} u^{0}\right\|_{X_{\varepsilon}^{\frac{1}{2}}}+\left\|\lambda^{0}\left(Q_{\varepsilon}-Q_{0}\right) u^{0}\right\|_{X_{\varepsilon}^{\frac{1}{2}}} \\
& \leq\left|\lambda^{\varepsilon} \lambda^{0}\right|\left\|A_{0}^{-1} Q_{0} u^{0}-A_{\varepsilon}^{-1} Q_{\varepsilon} u^{0}\right\|_{X_{\varepsilon}^{\frac{1}{2}}}+\left|\lambda^{0}\right| \|\left(Q_{\varepsilon}-Q_{0}\right) u_{X_{\varepsilon}^{0} \|_{X^{\frac{1}{2}}}}
\end{aligned}
$$

and

$$
\begin{aligned}
\left\|A_{0}^{-1} Q_{0} u^{0}-A_{\varepsilon}^{-1} Q_{\varepsilon} u^{0}\right\|_{X_{\varepsilon}^{\frac{1}{2}}} & =\left\|A_{0}^{-1} Q_{0} u^{0}-A_{\varepsilon}^{-1} Q_{0} u^{0}+A_{\varepsilon}^{-1} Q_{0} u^{0}-A_{\varepsilon}^{-1} Q_{\varepsilon} u^{0}\right\|_{X_{\varepsilon}^{\frac{1}{2}}} \\
& \leq\left\|\left(A_{0}^{-1}-A_{\varepsilon}^{-1}\right) Q_{0} u^{0}\right\|_{X_{\varepsilon}^{\frac{1}{2}}+\left\|A_{\varepsilon}^{-1}\left(Q_{0}-Q_{\varepsilon}\right) u^{0}\right\|_{X_{\varepsilon}^{\frac{1}{2}}}}
\end{aligned}
$$

Hence the estimate for $\left|\lambda^{\varepsilon}-\lambda^{0}\right|$ follows. 
In (CARBONE; RUAS-FILHO, 2006) it was proved that the eigenvalues of $-A_{\varepsilon}$ has a gap condition by characterizing these eigenvalues, that is, if $\sigma\left(-A_{\varepsilon}\right)=\left\{\mu_{i}^{\varepsilon}\right\}_{i=0}^{\infty}$ then

$$
\mu_{i}^{\varepsilon}=-\frac{1}{l^{2}} i^{2} \pi^{2}+o(i)
$$

as $i \rightarrow \infty$, where $l=\int_{0}^{1} p_{\varepsilon}(s)^{-\frac{1}{2}} d s$. Consequently $\lambda_{i}^{\varepsilon}-\lambda_{i+1}^{\varepsilon} \stackrel{i \rightarrow \infty}{\longrightarrow} \infty$.

Recall that we denote $\mathscr{E}_{\varepsilon}$ the set of the equilibria solutions of the $A_{\varepsilon}$ and we assume that $\mathscr{E} 0$ is composed of hyperbolic solutions, thus for $\varepsilon$ sufficiently small $\mathscr{E}_{\varepsilon}$ is composed of finite number of hyperbolic solutions. The rate of convergence of equilibrium points can be obtained as follows.

Theorem 6.3.2. Let $u_{*}^{0} \in \mathscr{E}_{0}$. Then for $\varepsilon$ sufficiently small (we still denote $\varepsilon \in\left(0, \varepsilon_{0}\right]$ ), there is $\delta>0$ such that the equation $A_{\varepsilon} u-f(u)=0$ has only solution $u_{*}^{\varepsilon} \in\left\{u \in X_{\mathcal{\varepsilon}}^{\frac{1}{2}} ;\left\|u-u_{*}^{0}\right\|_{X_{\varepsilon}^{\frac{1}{2}}} \leq \delta\right\}$. Moreover

$$
\left\|u_{*}^{\varepsilon}-u_{*}^{0}\right\|_{X_{\varepsilon}^{\frac{1}{2}}} \leq C\left(\left\|p_{\varepsilon}-p_{0}\right\|_{L^{\infty}\left(\Omega_{1}\right)}+\varepsilon\right)^{\frac{1}{2}}
$$

Proof. The proof is the same as given in (CARBONE; CARVALHO; SCHIABEL-SILVA, 2008). Here we just need to prove the estimates (6.8). We have $u_{*}^{\varepsilon}$ and $u_{*}^{0}$ given by

$$
u_{*}^{0}=\left(A_{0}+V_{0}\right)^{-1}\left[f\left(u_{*}^{0}\right)+V_{0} u_{*}^{0}\right] \quad \text { and } \quad u_{*}^{\varepsilon}=\left(A_{\varepsilon}+V_{0}\right)^{-1}\left[f\left(u_{*}^{\varepsilon}\right)+V_{0} u_{*}^{\varepsilon}\right],
$$

where $V_{0}=-f^{\prime}\left(u_{*}^{0}\right)$. Thus

$$
\begin{aligned}
\left\|u_{*}^{\varepsilon}-u_{*}^{0}\right\|_{X_{\varepsilon}^{\frac{1}{2}}} & \leq\left\|\left(A_{\varepsilon}+V_{0}\right)^{-1}\left[f\left(u_{*}^{\varepsilon}\right)+V_{0} u_{*}^{\varepsilon}\right]-\left(A_{0}+V_{0}\right)^{-1}\left[f\left(u_{*}^{0}\right)+V_{0} u_{*}^{0}\right]\right\|_{X_{\varepsilon}^{\frac{1}{2}}} \\
& \leq\left\|\left(A_{\varepsilon}+V_{0}\right)^{-1}\left[f\left(u_{*}^{\varepsilon}\right)-f\left(u_{*}^{0}\right)+V_{0}\left(u_{*}^{\varepsilon}-u_{*}^{0}\right)\right]\right\|_{X_{\varepsilon}^{\frac{1}{2}}} \\
& +\left\|\left[\left(A_{\varepsilon}+V_{0}\right)^{-1}-\left(A_{0}+V_{0}\right)^{-1}\right]\left[f\left(u_{*}^{0}\right)+V_{0} u_{*}^{0}\right]\right\|_{X_{\varepsilon}^{\frac{1}{2}}}
\end{aligned}
$$

We can prove that

$$
\left(A_{\varepsilon}+V_{0}\right)^{-1}-\left(A_{0}+V_{0}\right)^{-1}=\left[I-\left(A_{\varepsilon}+V_{0}\right)^{-1} V_{0}\right]\left(A_{\varepsilon}^{-1}-A_{0}^{-1}\right)\left[I-V_{0}\left(A_{0}+V_{0}\right)^{-1}\right],
$$

which implies $\left\|\left[\left(A_{\varepsilon}+V_{0}\right)^{-1}-\left(A_{0}+V_{0}\right)^{-1}\right]\left[f\left(u_{*}^{0}\right)+V_{0} u_{*}^{0}\right]\right\|_{X_{\varepsilon}^{\frac{1}{2}}} \leq C\left(\left\|p_{\varepsilon}-p_{0}\right\|_{L^{\infty}\left(\Omega_{1}\right)}+\varepsilon\right)^{\frac{1}{2}}$.

Now we denote $z^{\varepsilon}=f\left(u_{*}^{\varepsilon}\right)-f\left(u_{*}^{0}\right)+V_{0}\left(u_{*}^{\varepsilon}-u_{*}^{0}\right)$. Since $f$ is continuously differentiable, for all $\delta>0$ there is $\varepsilon$ sufficiently small such that $\left\|z^{\varepsilon}\right\|_{X_{\varepsilon}^{\frac{1}{2}}} \leq \delta\left\|u_{*}^{\varepsilon}-u_{*}^{0}\right\|_{X_{\varepsilon}^{\frac{1}{2}}}$, thus

$$
\left\|\left(A_{\varepsilon}+V_{0}\right)^{-1} z^{\varepsilon}\right\|_{X_{\varepsilon}^{\frac{1}{2}}} \leq \delta\left\|\left(A_{\varepsilon}+V_{0}\right)^{-1}\right\|_{\mathscr{L}\left(L_{\Omega_{0}}^{2}, X_{\varepsilon}^{\frac{1}{2}}\right)}\left\|u_{*}^{\varepsilon}-u_{*}^{0}\right\|_{X_{\varepsilon}^{\frac{1}{2}}} \cdot
$$

We choose $\delta$ sufficiently small such that $\delta\left\|\left(A_{\varepsilon}+V_{0}\right)^{-1}\right\|_{\mathscr{L}\left(L_{\Omega_{0}}^{2}, X_{\varepsilon}^{\frac{1}{2}}\right)} \leq \frac{1}{2}$, thus

$$
\left\|u_{*}^{\varepsilon}-u_{*}^{0}\right\|_{X_{\varepsilon}^{\frac{1}{2}}} \leq C\left(\left\|p_{\varepsilon}-p_{0}\right\|_{L^{\infty}\left(\Omega_{1}\right)}+\varepsilon\right)^{\frac{1}{2}}+\frac{1}{2}\left\|u_{*}^{\varepsilon}-u_{*}^{0}\right\|_{X_{\varepsilon}^{\frac{1}{2}}} .
$$


Corollary 6.3.3. The family $\left\{\mathscr{E}_{\varepsilon}\right\}_{\varepsilon \in\left(0, \varepsilon_{0}\right]}$ is continuous at $\varepsilon=0$. Moreover if $\mathscr{E}_{0}=\left\{u_{*}^{0,1}, \ldots, u_{*}^{0, k}\right\}$ then for $\varepsilon$ sufficiently small, $\mathscr{E}_{\varepsilon}=\left\{u_{*}^{\varepsilon, 1}, \ldots, u_{*}^{\varepsilon, k}\right\}$ and

$$
\left\|u_{*}^{\varepsilon, i}-u_{*}^{0, i}\right\|_{X_{\varepsilon}^{\frac{1}{2}}} \leq C\left(\left\|p_{\varepsilon}-p_{0}\right\|_{L^{\infty}\left(\Omega_{1}\right)}+\varepsilon\right)^{\frac{1}{2}}, \quad i=1, \ldots, k .
$$

\subsection{Rate of Convergence of Invariant Manifolds}

In this section we characterize the invariant manifolds $\mathscr{M}_{\varepsilon}$ locally as a graph of a Lipschitz function, and we guarantee that $\mathscr{M}_{\varepsilon}$ approaches to the invariant manifold $\mathscr{M}_{0}$ when the parameter $\varepsilon$ goes to zero. This result will be fundamental to reduce the study of the asymptotic dynamics of the problem (6.4) to a finite dimension.

We will follows the works (CARBONE; RUAS-FILHO, 2006) and (CARVALHO et al., 2010).

The spectrum of $-A_{\varepsilon}, \varepsilon \in\left[0, \varepsilon_{0}\right]$, ordered and counting multiplicity is given by

$$
\ldots-\lambda_{m}^{\varepsilon}<0<-\lambda_{m-1}^{\varepsilon}<\ldots<-\lambda_{0}^{\varepsilon}
$$

with $\left\{\varphi_{i}^{\varepsilon}\right\}_{i=0}^{\infty}$ the eigenfunctions related. We consider the spectral projection onto the space generated by the first $m$ eigenvalues, that is, if $\Gamma$ is an appropriated closed curve in $\rho\left(-A_{0}\right)$ around $\left\{-\lambda_{0}^{0}, \ldots,-\lambda_{m-1}^{0}\right\}$, then

$$
Q_{\varepsilon}=\frac{1}{2 \pi i} \int_{\Gamma}\left(\mu+A_{\varepsilon}\right)^{-1} d \mu, \quad \varepsilon \in\left[0, \varepsilon_{0}\right] .
$$

We observe that $Q_{\varepsilon}$ is a projection of finite rank and then there is an isomorphism from $Q_{\varepsilon} X_{\mathcal{\varepsilon}}^{\frac{1}{2}}=$ $\operatorname{span}\left[\varphi_{0}^{\varepsilon}, \ldots, \varphi_{m-1}^{\varepsilon}\right]$ onto $\mathbb{R}^{m}$. Thus we can decompose $X_{\varepsilon}^{\frac{1}{2}}=Y_{\varepsilon} \oplus Z_{\varepsilon}$, where $Y_{\varepsilon}=Q_{\varepsilon} X_{\varepsilon}^{\frac{1}{2}}$ and $Z_{\varepsilon}=\left(I-Q_{\varepsilon}\right) X_{\varepsilon}^{\frac{1}{2}}$ and we define $A_{\varepsilon}^{+}=\left.A_{\varepsilon}\right|_{Y_{\varepsilon}}$ and $A_{\varepsilon}^{-}=\left.A_{\varepsilon}\right|_{Z_{\varepsilon}} \varepsilon \in\left[0, \varepsilon_{0}\right]$. The following estimates are valid.

(i) $\left\|e^{-A_{\varepsilon}^{+} t} z\right\|_{X_{\varepsilon}^{\frac{1}{2}}} \leq M e^{-\beta t}\|z\|_{X_{\varepsilon}^{\frac{1}{2}}}, \quad t \leq 0, \quad z \in Y_{\varepsilon}$,

(ii) $\left\|e^{-A_{\varepsilon}^{-} t} z\right\|_{X_{\varepsilon}^{\frac{1}{2}}} \leq M e^{-\gamma t}\|z\|_{X_{\varepsilon}^{\frac{1}{2}}}, \quad t>0, \quad z \in Z_{\varepsilon}$,

(iii) $\left\|e^{-A_{\varepsilon}^{+} t}-e^{A_{0}^{+} t}\right\|_{\mathscr{L}\left(L_{\Omega_{0}}^{2}, X_{\varepsilon}^{\frac{1}{2}}\right)} \leq M e^{-\beta t}\left(\left\|p_{\varepsilon}-p_{0}\right\|_{L^{\infty}\left(\Omega_{1}\right)}+\varepsilon\right)^{\frac{1}{2}}, \quad t \leq 0$,

(iv) $\left\|e^{-A_{\varepsilon}^{-} t}-e^{A_{0}^{-} t}\right\|_{\mathscr{L}\left(L_{\Omega_{0}}^{2}, X_{\varepsilon}^{\frac{1}{2}}\right)} \leq M e^{-\gamma t} l_{\varepsilon}(t), \quad t>0$.

where $l_{\varepsilon}(t)=\max \left\{t^{-\frac{1}{2}},\left(\left\|p_{\varepsilon}-p_{0}\right\|_{L^{\infty}\left(\Omega_{1}\right)}+\varepsilon\right)^{\frac{1}{2}} t^{-1}\right\}, \gamma=\gamma(\varepsilon)=\lambda_{m+1}^{\varepsilon}, \beta=\lambda_{m}^{0}+1$, and $M$ is a positive constant independent of $\varepsilon$. 
Theorem 6.4.1. For $\varepsilon$ sufficiently small there is an invariant manifold $\mathscr{M}_{\varepsilon}$ for (6.4) given by

$$
\mathscr{M}_{\varepsilon}=\left\{u^{\varepsilon} \in X_{\varepsilon}^{\frac{1}{2}} ; u^{\varepsilon}=Q_{\varepsilon} u^{\varepsilon}+s_{*}^{\varepsilon}\left(Q_{\varepsilon} u^{\varepsilon}\right)\right\}, \quad \varepsilon \in\left[0, \varepsilon_{0}\right]
$$

where $s_{*}^{\varepsilon}: Y_{\varepsilon} \rightarrow Z_{\varepsilon}$ is a Lipschitz continuous map satisfying

$$
\left\|s_{*}^{\varepsilon}-s_{*}^{0}\right\|\left\|=\sup _{v \in Y_{0}}\right\| s_{*}^{\varepsilon}(v)-s_{*}^{0}(v) \|_{X_{\varepsilon}^{\frac{1}{2}}} \leq C\left(\left\|p_{\varepsilon}-p_{0}\right\|_{L^{\infty}\left(\Omega_{1}\right)}+\varepsilon\right)^{\frac{1}{2}}\left|\log \left(\left\|p_{\varepsilon}-p_{0}\right\|_{L^{\infty}\left(\Omega_{1}\right)}+\varepsilon\right)\right|
$$

for some constant $C$ independent of $\varepsilon$. The invariant manifold $\mathscr{M}_{\varepsilon}$ is exponentially attracting and the global attractor $\mathscr{A}_{\varepsilon}$ of the problem (6.4) lies in $\mathscr{M}_{\varepsilon}$. The flow on $\mathscr{A}_{\varepsilon}$ is given by

$$
u^{\varepsilon}(t)=v^{\varepsilon}(t)+s_{*}^{\varepsilon}\left(v^{\varepsilon}(t)\right), \quad t \in \mathbb{R},
$$

where $v^{\varepsilon}(t)$ satisfies

$$
\dot{v}^{\varepsilon}+A_{\varepsilon}^{+} v^{\varepsilon}=Q_{\varepsilon} f\left(v^{\varepsilon}+s_{*}^{\varepsilon}\left(v^{\varepsilon}(t)\right)\right)
$$

Proof. Given $L, \Delta>0$ we consider the set

$$
\Sigma_{\varepsilon}=\left\{s^{\varepsilon}: Y_{\varepsilon} \rightarrow Z_{\varepsilon} ;\left\|s^{\varepsilon}\right\| \leq D \text { and }\left\|s^{\varepsilon}(v)-s^{\varepsilon}(\tilde{v})\right\|_{X_{\varepsilon}^{\frac{1}{2}}} \leq \Delta\|v-\tilde{v}\|_{X_{\varepsilon}^{\frac{1}{2}}}\right\} .
$$

It is not difficult to see that $\left(\Sigma_{\varepsilon},\|\mid\| \|\right)$ is a complete metric space. We write the solution $u^{\varepsilon}$ of (6.4) as $u^{\varepsilon}=v^{\varepsilon}+z^{\varepsilon}$, with $v^{\varepsilon} \in Y_{\varepsilon}$ and $z^{\varepsilon} \in Z_{\varepsilon}$ and since $Q_{\varepsilon}$ and $I-Q_{\varepsilon}$ commute with $A_{\varepsilon}$, we can write

$$
\left\{\begin{array}{l}
v_{t}^{\varepsilon}+A_{\mathcal{\varepsilon}}^{+} v^{\varepsilon}=Q_{\varepsilon} f\left(v^{\varepsilon}+z^{\varepsilon}\right):=H_{\varepsilon}\left(v^{\varepsilon}, z^{\varepsilon}\right) \\
z_{t}^{\varepsilon}+A_{\varepsilon}^{-} z^{\varepsilon}=\left(I-Q_{\varepsilon}\right) f\left(v^{\varepsilon}+z^{\varepsilon}\right):=G_{\varepsilon}\left(v^{\varepsilon}, z^{\varepsilon}\right) .
\end{array}\right.
$$

By assumption there is a certain $\rho>0$ such that for all $v^{\varepsilon}, \tilde{v}^{\varepsilon} \in Y_{\varepsilon}$ and $z^{\varepsilon}, \tilde{z}^{\varepsilon} \in Z_{\varepsilon}$,

$$
\begin{aligned}
& \left\|H_{\varepsilon}\left(v^{\varepsilon}, z^{\varepsilon}\right)\right\|_{X_{\varepsilon}^{\frac{1}{2}}} \leq \rho,\left\|G_{\varepsilon}\left(v^{\varepsilon}, z^{\varepsilon}\right)\right\|_{X_{\varepsilon}^{\frac{1}{2}}} \leq \rho, \\
& \left\|H_{\varepsilon}\left(v^{\varepsilon}, z^{\varepsilon}\right)-H_{\varepsilon}\left(\tilde{v}^{\varepsilon}, \tilde{z}^{\varepsilon}\right)\right\|_{X_{\varepsilon}^{\frac{1}{2}}} \leq \rho\left(\left\|v^{\varepsilon}-\tilde{v}_{\varepsilon}\right\|_{X_{\varepsilon}^{\frac{1}{2}}}+\left\|z^{\varepsilon}-\tilde{z}_{\varepsilon}\right\|_{X_{\varepsilon}^{\frac{1}{2}}}\right), \\
& \left\|G_{\varepsilon}\left(v^{\varepsilon}, z^{\varepsilon}\right)-G_{\varepsilon}\left(\tilde{v}^{\varepsilon}, \tilde{z}^{\varepsilon}\right)\right\|_{X_{\varepsilon}^{\frac{1}{2}}} \leq \rho\left(\left\|v^{\varepsilon}-\tilde{v}_{\varepsilon}\right\|_{X_{\varepsilon}^{\frac{1}{2}}}+\left\|z^{\varepsilon}-\tilde{z}_{\varepsilon}\right\|_{X_{\varepsilon}^{\frac{1}{2}}}\right) .
\end{aligned}
$$

Also, for $\varepsilon$ sufficiently small, we can choose $\rho$ such that

$$
\begin{aligned}
& \rho M \gamma^{-1} \leq D, 0 \leq \gamma-\beta-\rho M(1+\Delta), \frac{\rho M^{2}(1+\Delta)}{\gamma-\beta-\rho M(1+\Delta)} \leq \Delta, \\
& \rho M \gamma^{-1}+\frac{\rho^{2} M^{2}(1+\Delta) \beta^{-1}}{\gamma-\beta-\rho M(1+\Delta)} \leq \frac{1}{2}, L=\left[\rho M+\frac{\rho^{2} M^{2}(1+\Delta)(1+M)}{\gamma-\beta-\rho M(1+\Delta)}\right], \gamma-L>0 .
\end{aligned}
$$

We will divide the proof in three parts. 
Part 1(Existence) Let $s^{\varepsilon} \in \Sigma_{\varepsilon}$ and $v^{\varepsilon}(t)=v^{\varepsilon}\left(t, \tau, \eta, s^{\varepsilon}\right)$ be the solution of

$$
\left\{\begin{array}{l}
v_{t}^{\varepsilon}+A_{\mathcal{\varepsilon}}^{+} v^{\varepsilon}=H_{\varepsilon}\left(v^{\varepsilon}, s^{\varepsilon}\left(v^{\varepsilon}\right)\right), \quad t<\tau \\
v^{\varepsilon}(\tau)=\eta
\end{array}\right.
$$

We define $\Phi_{\varepsilon}: \Sigma_{\varepsilon} \rightarrow \Sigma_{\varepsilon}$ by

$$
\Phi_{\varepsilon}\left(s^{\varepsilon}\right)(\eta)=\int_{-\infty}^{\tau} e^{-A_{\varepsilon}^{-}(\tau-r)} G_{\mathcal{\varepsilon}}\left(v^{\varepsilon}(r), s^{\varepsilon}\left(v^{\varepsilon}(r)\right)\right) d r
$$

Then

$$
\left\|\Phi_{\varepsilon}\left(s^{\varepsilon}\right)(\eta)\right\|_{X_{\varepsilon}^{\frac{1}{2}}} \leq \rho M \int_{-\infty}^{\tau} e^{-\gamma(\tau-r)} d r=\rho M \gamma^{-1} \leq D .
$$

For $s^{\varepsilon}, \tilde{s}^{\varepsilon} \in \Sigma_{\varepsilon}, \eta, \tilde{\eta} \in Y_{\mathcal{\varepsilon}}, v^{\varepsilon}(t)=v^{\varepsilon}\left(t, \tau, \eta, s^{\varepsilon}\right)$ and $\tilde{v}^{\varepsilon}(t)=\tilde{v}^{\varepsilon}\left(t, \tau, \tilde{\eta}, \tilde{s}^{\varepsilon}\right)$ we have

$$
\begin{aligned}
v^{\varepsilon}(t)-\tilde{v}^{\varepsilon}(t)=e^{-A_{\varepsilon}^{+}(t-\tau)}(\eta & -\tilde{\eta}) \\
& +\int_{\tau}^{t} e^{-A_{\varepsilon}^{+}(t-r)}\left[H_{\varepsilon}\left(v^{\varepsilon}(r), s^{\varepsilon}\left(v^{\varepsilon}(r)\right)\right)-H_{\varepsilon}\left(\tilde{v}^{\varepsilon}(r), \tilde{s}^{\varepsilon}\left(\tilde{v}^{\varepsilon}(r)\right)\right)\right] d r .
\end{aligned}
$$

Thus,

$$
\begin{aligned}
& \left\|v^{\varepsilon}(t)-\tilde{v}^{\varepsilon}(t)\right\|_{X_{\varepsilon}^{\frac{1}{2}}} \leq M e^{-\beta(t-\tau)}\|\eta-\tilde{\eta}\|_{X_{\varepsilon}^{\frac{1}{2}}} \\
& +M \int_{t}^{\tau} e^{-\beta(t-r)}\left\|H_{\mathcal{E}}\left(v^{\varepsilon}(r), s^{\varepsilon}\left(v^{\varepsilon}(r)\right)\right)-H_{\mathcal{E}}\left(\tilde{v}^{\varepsilon}(r), \tilde{s}^{\varepsilon}\left(\tilde{v}^{\varepsilon}(r)\right)\right)\right\|_{X_{\varepsilon}^{\frac{1}{2}}} d r \\
& \leq M e^{-\beta(t-\tau)}\|\eta-\tilde{\eta}\|_{X_{\varepsilon}^{\frac{1}{2}}} \\
& +\rho M \int_{t}^{\tau} e^{-\beta(t-r)}\left[\left\|v^{\varepsilon}(r)-\tilde{v}^{\varepsilon}(r)\right\|_{X_{\varepsilon}^{\frac{1}{2}}}+\left\|s^{\varepsilon}\left(v^{\varepsilon}(r)\right)-\tilde{s}^{\varepsilon}\left(\tilde{v}^{\varepsilon}(r)\right)\right\|_{X_{\varepsilon}^{\frac{1}{2}}}\right] d r \\
& \leq M e^{-\beta(t-\tau)}\|\eta-\tilde{\eta}\|_{X_{\varepsilon}^{\frac{1}{2}}} \\
& +\rho M \int_{t}^{\tau} e^{-\beta(t-r)}\left[(1+\Delta)\left\|v^{\varepsilon}(r)-\tilde{v}^{\varepsilon}(r)\right\|_{X_{\varepsilon}^{\frac{1}{2}}}+\left\|s^{\varepsilon}\left(\tilde{v}^{\varepsilon}(r)\right)-\tilde{s}^{\mathcal{E}}\left(\tilde{v}^{\varepsilon}(r)\right)\right\|_{X_{\varepsilon}^{\frac{1}{2}}}\right] d r \\
& \leq M e^{-\beta(t-\tau)}\|\eta-\tilde{\eta}\|_{X_{\varepsilon}^{\frac{1}{2}}} \\
& +\rho M(1+\Delta) \int_{t}^{\tau} e^{-\beta(t-r)}\left\|v^{\varepsilon}(r)-\tilde{v}^{\varepsilon}(r)\right\|_{X_{\varepsilon}^{\frac{1}{2}}} d r+\rho M\left\|s^{\varepsilon}-\tilde{s}^{\varepsilon}\right\| \int_{t}^{\tau} e^{-\beta(t-r)} d r \\
& \leq M e^{-\beta(t-\tau)}\|\eta-\tilde{\eta}\|_{X_{\varepsilon}^{\frac{1}{2}}} \\
& +\rho M(1+\Delta) \int_{t}^{\tau} e^{-\beta(t-r)}\left\|v^{\varepsilon}(r)-\tilde{v}^{\varepsilon}(r)\right\|_{X_{\varepsilon}^{\frac{1}{2}}} d r+\rho M \beta^{-1}\left\|s^{\varepsilon}-\tilde{s}^{\varepsilon}\right\| e^{-\beta(t-\tau)} .
\end{aligned}
$$

By Gronwall's inequality,

$$
\left\|v^{\varepsilon}(t)-\tilde{v}^{\varepsilon}(t)\right\|_{X_{\varepsilon}^{\frac{1}{2}}} \leq\left[M\|\eta-\tilde{\eta}\|_{X_{\varepsilon}^{\frac{1}{2}}}+\rho M \beta^{-1}\left\|s^{\varepsilon}-\tilde{s}^{\varepsilon}\right\| \|\right] e^{[\rho M(1+\Delta)+\beta](\tau-t)}
$$


Thus

$$
\begin{aligned}
\| \Phi_{\varepsilon}\left(s^{\varepsilon}\right)(\eta) & -\Phi_{\varepsilon}\left(\tilde{s}^{\varepsilon}\right)(\tilde{\eta}) \|_{X_{\varepsilon}^{\frac{1}{2}}} \\
& \leq \int_{-\infty}^{\tau}\left\|e^{-\bar{A}_{\varepsilon}^{-}(\tau-r)}\left[G_{\varepsilon}\left(v^{\varepsilon}(r), s^{\varepsilon}\left(v^{\varepsilon}(r)\right)\right)-G_{\varepsilon}\left(\tilde{v}^{\varepsilon}(r), \tilde{s}^{\varepsilon}\left(\tilde{v}^{\varepsilon}(r)\right)\right)\right]\right\|_{X_{\varepsilon}^{\frac{1}{2}}} d r \\
& \leq \frac{\rho M^{2}(1+\Delta)}{\gamma-\beta-\rho M(1+\Delta)}\|\eta-\tilde{\eta}\|_{X_{\varepsilon}^{\frac{1}{2}}}+\frac{\rho^{2} M^{2}(1+\Delta) \beta^{-1}}{\gamma-\beta-\rho M(1+\Delta)}\left\|s^{\varepsilon}-\tilde{s}^{\varepsilon}\right\| .
\end{aligned}
$$

Therefore $\Phi_{\varepsilon}$ is a contraction on $\Sigma_{\varepsilon}$, hence there is a unique $s_{*}^{\varepsilon} \in \Sigma_{\varepsilon}$ which is a fixed point of $\Phi_{\varepsilon}$.

Now, let $\left(\bar{v}^{\varepsilon}, \bar{z}^{\varepsilon}\right) \in \mathscr{M}_{\varepsilon}, \bar{z}^{\varepsilon}=s_{*}^{\varepsilon}\left(\bar{v}^{\varepsilon}\right)$ and let $v_{s_{*}}^{\varepsilon}(t)$ be the solution of

$$
\left\{\begin{array}{l}
v_{t}^{\varepsilon}+A_{\varepsilon}^{+} v^{\varepsilon}=H_{\varepsilon}\left(v^{\varepsilon}, s_{*}^{\varepsilon}\left(v^{\varepsilon}\right)\right), \quad t<\tau \\
v^{\varepsilon}(0)=\bar{v}^{\varepsilon}
\end{array}\right.
$$

Thus, $\left\{\left(v_{s_{*}}^{\varepsilon}(t), s_{*}^{\varepsilon}\left(v_{s_{*}}^{\varepsilon}(t)\right)\right\}_{t \in \mathbb{R}}\right.$ defines a curve on $\mathscr{M}_{\varepsilon}$. But the only solution of equation

$$
z_{t}^{\varepsilon}+A_{\varepsilon}^{-} z^{\varepsilon}=G_{\varepsilon}\left(v_{s_{*}}^{\varepsilon}(t), s_{*}^{\varepsilon}\left(v_{s_{*}}^{\varepsilon}(t)\right)\right)
$$

which stays bounded when $t \rightarrow-\infty$ is given by

$$
z_{s_{*}}^{\varepsilon}=\int_{-\infty}^{t} e^{-A_{\varepsilon}^{-}(t-r)} G_{\varepsilon}\left(v_{s_{*}}^{\varepsilon}(t), s_{*}^{\varepsilon}\left(v_{s_{*}}^{\varepsilon}(t)\right)\right) d r=s_{*}^{\varepsilon}\left(v_{s_{*}}^{\varepsilon}(t)\right) .
$$

Therefore $\left(v_{s_{*}}^{\varepsilon}(t), s_{*}^{\varepsilon}\left(v_{s_{*}}^{\varepsilon}(t)\right)\right.$ is a solution of (6.10) through $\left(\bar{v}^{\varepsilon}, \bar{z}^{\varepsilon}\right)$ and thus $\mathscr{M}_{\varepsilon}$ is a invariant manifold for (6.4).

Part 2(Estimate) Now we will prove the estimate (6.9). For $\eta \in Y_{0}$, we have

$$
\begin{aligned}
\left\|s_{*}^{\varepsilon}(\eta)-s_{*}^{0}(\eta)\right\|_{X_{\varepsilon}^{\frac{1}{2}}} & \leq \int_{-\infty}^{\tau}\left\|e^{-A_{\varepsilon}^{-}(\tau-r)} G_{\mathcal{\varepsilon}}\left(v^{\varepsilon}, s_{*}^{\varepsilon}\left(v^{\varepsilon}\right)\right)-e^{-A_{0}^{-}(\tau-r)} G_{0}\left(v^{0}, s_{*}^{0}\left(v^{0}\right)\right)\right\|_{X_{\varepsilon}^{\frac{1}{2}}} d r \\
& \leq \int_{-\infty}^{\tau}\left\|e^{-A_{\varepsilon}^{-}(\tau-r)} G_{\mathcal{\varepsilon}}\left(v^{\varepsilon}, s_{*}^{\varepsilon}\left(v^{\varepsilon}\right)\right)-e^{-A_{\varepsilon}^{-}(\tau-r)} G_{\mathcal{\varepsilon}}\left(v^{0}, s_{*}^{0}\left(v^{0}\right)\right)\right\|_{X_{\varepsilon}^{\frac{1}{2}}} d r \\
& +\int_{-\infty}^{\tau}\left\|e^{-A_{\varepsilon}^{-}(\tau-r)} G_{\varepsilon}\left(v^{0}, s_{*}^{0}\left(v^{0}\right)\right)-e^{-A_{\varepsilon}^{-}(\tau-r)} G_{0}\left(v^{0}, s_{*}^{0}\left(v^{0}\right)\right)\right\|_{X_{\varepsilon}^{\frac{1}{2}}} d r \\
& +\int_{-\infty}^{\tau}\left\|e^{-A_{\varepsilon}^{-}(\tau-r)} G_{0}\left(v^{0}, s_{*}^{0}\left(v^{0}\right)\right)-e^{-A_{0}^{-}(\tau-r)} G_{0}\left(v^{0}, s_{*}^{0}\left(v^{0}\right)\right)\right\|_{X_{\varepsilon}^{\frac{1}{2}}} d r .
\end{aligned}
$$

If we denote the last three integrals for $I_{1}, I_{2}$ and $I_{3}$ respectively, we have

$$
\begin{aligned}
I_{1} & \leq \rho M \int_{-\infty}^{\tau} e^{-\gamma(\tau-r)}\left[(1+\Delta)\left\|v^{\varepsilon}-v^{0}\right\|_{X_{\varepsilon}^{\frac{1}{2}}}+\left\|s_{*}^{\varepsilon}-s_{*}^{0}\right\|\right] d r \\
& \leq \rho M(1+\Delta) \int_{-\infty}^{\tau} e^{-\gamma(\tau-r)}\left\|v^{\varepsilon}-v^{0}\right\|_{X_{\varepsilon}^{\frac{1}{2}}} d r+\rho M\left\|s_{*}^{\varepsilon}-s_{*}^{0}\right\| \int_{-\infty}^{\tau} e^{-\gamma(\tau-r)} d r \\
& =\rho M \gamma^{-1}\left\|s_{*}^{\varepsilon}-s_{*}^{0}\right\|+\rho M(1+\Delta) \int_{-\infty}^{\tau} e^{-\gamma(\tau-r)}\left\|v^{\varepsilon}-v^{0}\right\|_{X_{\varepsilon}^{\frac{1}{2}}} d r .
\end{aligned}
$$

For $I_{2}$ we have

$$
G_{\varepsilon}\left(v^{0}, s_{*}^{0}\left(v^{0}\right)\right)-G_{0}\left(v^{0}, s_{*}^{0}\left(v^{0}\right)\right)=\left(Q_{\varepsilon}-Q_{0}\right) f\left(v^{0}+s_{*}^{0}\left(v^{0}\right)\right)
$$


and if we denote $\tau(\varepsilon)=\left(\left\|p_{\varepsilon}-p_{0}\right\|_{L^{\infty}\left(\Omega_{1}\right)}+\varepsilon\right)^{\frac{1}{2}}$, then $I_{2} \leq C \tau(\varepsilon)$. And for $I_{3}$, we have

$$
I_{3} \leq \int_{-\infty}^{\tau} l_{\varepsilon}(\tau-r) e^{-\bar{\gamma}(\tau-r)} d r \leq C \tau(\varepsilon)|\log (\tau(\varepsilon))|
$$

where we have used the Lemma 3.10 in (SANTAMARIA; ARRIETA, 2014). Thus

$$
\begin{aligned}
\left\|s_{*}^{\varepsilon}(\eta)-s_{*}^{0}(\eta)\right\|_{X_{\varepsilon}^{\frac{1}{2}}} \leq C \tau(\varepsilon) & |\log (\tau(\varepsilon))| \\
& +\rho M \gamma^{-1}\left\|s_{*}^{\varepsilon}-s_{*}^{0}\right\|+\rho M(1+\Delta) \int_{-\infty}^{\tau} e^{-\gamma(\tau-r)}\left\|v^{\varepsilon}-v^{0}\right\|_{X_{\varepsilon}^{\frac{1}{2}}} d r .
\end{aligned}
$$

But,

$$
\begin{aligned}
\left\|v^{\varepsilon}(t)-v^{0}(t)\right\|_{X_{\varepsilon}^{\frac{1}{2}}} & \leq\left\|\left(e^{-A_{\varepsilon}^{+}(t-\tau)}-e^{-A_{0}^{+}(t-\tau)}\right) \eta\right\| \\
& +\int_{t}^{\tau}\left\|e^{-A_{\varepsilon}^{+}(t-r)} H_{\mathcal{\varepsilon}}\left(v^{\varepsilon}, s_{*}^{\varepsilon}\left(v^{\varepsilon}\right)\right)-e^{-A_{\varepsilon}^{+}(t-r)} H_{\mathcal{\varepsilon}}\left(v^{0}, s_{*}^{0}\left(v^{0}\right)\right)\right\|_{X_{\varepsilon}^{\frac{1}{2}}} d r \\
& +\int_{t}^{\tau}\left\|e^{-A_{\varepsilon}^{+}(t-r)} H_{\mathcal{\varepsilon}}\left(v^{0}, s_{*}^{0}\left(v^{0}\right)\right)-e^{-A_{\varepsilon}^{+}(t-r)} H_{0}\left(v^{0}, s_{*}^{0}\left(v^{0}\right)\right)\right\|_{X_{\varepsilon}^{\frac{1}{2}}} d r \\
& +\int_{t}^{\tau}\left\|e^{-A_{\varepsilon}^{+}(t-r)} H_{0}\left(v^{0}, s_{*}^{0}\left(v^{0}\right)\right)-e^{-A_{0}^{+}(t-r)} H_{0}\left(v^{0}, s_{*}^{0}\left(v^{0}\right)\right)\right\|_{X_{\varepsilon}^{\frac{1}{2}}} d r
\end{aligned}
$$

With the same argument used earlier, we have

$$
\begin{aligned}
\left\|v^{\varepsilon}(t)-v^{0}(t)\right\|_{X_{\varepsilon}^{\frac{1}{2}}} & \leq C \tau(\varepsilon) \int_{t}^{\tau} e^{-\beta(t-r)} d r \\
& +\rho M\left\|s_{*}^{\varepsilon}-s_{*}^{0}\right\| \int_{t}^{\tau} e^{-\beta(t-r)} d r+\rho M(1+\Delta) \int_{t}^{\tau} e^{-\beta(t-r)}\left\|v^{\varepsilon}-v^{0}\right\|_{X_{\varepsilon}^{\frac{1}{2}}} d r .
\end{aligned}
$$

By Gronwall's inequality,

$$
\left\|v^{\varepsilon}(t)-v^{0}(t)\right\|_{X_{\varepsilon}^{\frac{1}{2}}} \leq\left[C \tau(\varepsilon)|\log (\tau(\varepsilon))|+\rho M \beta^{-1}\left\|s_{*}^{\varepsilon}-s_{*}^{0}\right\|\right] \mid e^{[\rho M(1+\Delta)+\beta](\tau-t)},
$$

thus

$$
\begin{aligned}
& \left\|s_{*}^{\varepsilon}(\eta)-s_{*}^{0}(\eta)\right\|_{X_{\varepsilon}^{\frac{1}{2}}} \leq C \tau(\varepsilon)|\log (\tau(\varepsilon))|+\rho M \gamma^{-1}\left\|s_{*}^{\varepsilon}-s_{*}^{0}\right\| \| \\
& +\rho M(1+\Delta) \int_{-\infty}^{\tau} e^{-\gamma(\tau-r)} e^{[\rho M(1+\Delta)+\beta](\tau-r)} d r\left[C \tau(\varepsilon)|\log (\tau(\varepsilon))|+\rho M \beta^{-1}\left\|s_{*}^{\varepsilon}-s_{*}^{0}\right\| \|\right] \\
& \leq C \tau(\varepsilon)|\log (\tau(\varepsilon))|+\left[\rho M \gamma^{-1}+\frac{\rho^{2} M^{2}(1+\Delta) \beta^{-1}}{\gamma-\beta-\rho M(1+\Delta)}\right]\left\|s_{*}^{\varepsilon}-s_{*}^{0}\right\| \|
\end{aligned}
$$

which implies $\left\|s_{*}^{\varepsilon}-s_{*}^{0}\right\||\leq C \tau(\varepsilon)| \log (\tau(\varepsilon)) \mid$.

Part 3(Exponential attraction) It remains shown that $\mathscr{M}_{\varepsilon}$ is exponentially attracting and $\mathscr{A}_{\mathcal{\varepsilon}} \subset \mathscr{M}_{\varepsilon}$. Let $\left(v^{\varepsilon}, z^{\varepsilon}\right) \in Y_{\varepsilon} \oplus Z_{\varepsilon}$ be the solution of (6.10) and define $\xi^{\varepsilon}(t)=z^{\varepsilon}-s_{*}^{\varepsilon}\left(v^{\varepsilon}(t)\right)$ and consider $y^{\varepsilon}(r, t), r \leq t, t \geq 0$, the solution of

$$
\left\{\begin{array}{l}
y_{t}^{\varepsilon}+A_{\varepsilon}^{+} y^{\varepsilon}=H_{\varepsilon}\left(y^{\varepsilon}, s_{*}^{\varepsilon}\left(y^{\varepsilon}\right)\right), \quad r \leq t \\
y^{\varepsilon}(t, t)=v^{\varepsilon}(t) .
\end{array}\right.
$$


Thus,

$$
\begin{aligned}
\left\|y^{\varepsilon}(r, t)-v^{\varepsilon}(r)\right\|_{X_{\varepsilon}^{\frac{1}{2}}} & \\
& =\left\|\int_{t}^{r} e^{-A_{\varepsilon}^{+}(r-\theta)}\left[H_{\varepsilon}\left(y^{\varepsilon}(\theta, t), s_{*}^{\varepsilon}\left(y^{\varepsilon}(\theta, t)\right)\right)-H_{\varepsilon}\left(v^{\varepsilon}(\theta), z^{\varepsilon}(\theta)\right)\right] d \theta\right\|_{X_{\varepsilon}^{\frac{1}{2}}} \\
& \leq \rho M \int_{r}^{t} e^{-\beta(r-\theta)}\left[(1+\Delta)\left\|y^{\varepsilon}(\theta, t)-v^{\varepsilon}(\theta)\right\|_{X_{\varepsilon}^{\frac{1}{2}}}+\left\|\xi^{\varepsilon}(\theta)\right\|_{X_{\varepsilon}^{\frac{1}{2}}}\right] d \theta .
\end{aligned}
$$

By Gronwall's inequality

$$
\left\|y^{\varepsilon}(r, t)-v^{\varepsilon}(r)\right\|_{X_{\varepsilon}^{\frac{1}{2}}} \leq \rho M \int_{r}^{t} e^{-(-\beta-\rho M(1+\Delta))(\theta-r)}\left\|\xi^{\varepsilon}(\theta)\right\|_{X_{\varepsilon}^{\frac{1}{2}}} d \theta \quad r \leq t .
$$

Now we take $t_{0} \in[r, t]$ and then

$$
\begin{aligned}
\| y^{\varepsilon}(r, t) & -y^{\varepsilon}\left(r, t_{0}\right) \|_{X_{\varepsilon}^{\frac{1}{2}}} \\
& =\left\|e^{-A_{\varepsilon}^{+}\left(r-t_{0}\right)}\left[y\left(t_{0}, t\right)-v^{\varepsilon}\left(t_{0}\right)\right]\right\|_{X_{\varepsilon}^{\frac{1}{2}}} \\
& +\left\|\int_{t_{0}}^{r} e^{-A_{\varepsilon}^{+}(r-\theta)}\left[H_{\varepsilon}\left(y^{\varepsilon}(\theta, t), s_{*}^{\varepsilon}\left(y^{\varepsilon}(\theta, t)\right)\right)-H_{\varepsilon}\left(y^{\varepsilon}\left(\theta, t_{0}\right), s_{*}^{\varepsilon}\left(y^{\varepsilon}\left(\theta, t_{0}\right)\right)\right)\right] d \theta\right\|_{X_{\varepsilon}^{\frac{1}{2}}} \\
& \leq \rho M^{2} e^{-\beta\left(r-t_{0}\right)} \int_{t_{0}}^{t} e^{-(-\beta-\rho M(1+\Delta))\left(\theta-t_{0}\right)}\left\|\xi^{\varepsilon}(\theta)\right\|_{X_{\varepsilon}^{\frac{1}{2}}} d \theta \\
& +\rho M \int_{r}^{t_{0}} e^{-\beta(r-\theta)}(1+\Delta)\left\|y^{\varepsilon}(\theta, t)-y^{\varepsilon}\left(\theta, t_{0}\right)\right\|_{X_{\varepsilon}^{\frac{1}{2}}} d \theta .
\end{aligned}
$$

By Gronwall's inequality

$$
\left\|y^{\varepsilon}(r, t)-y^{\varepsilon}\left(r, t_{0}\right)\right\|_{X_{\varepsilon}^{\frac{1}{2}}} \leq \rho M^{2} \int_{t_{0}}^{t} e^{-(-\beta-\rho M(1+\Delta))(\theta-r)}\left\|\xi^{\varepsilon}(\theta)\right\|_{X_{\varepsilon}^{\frac{1}{2}}} d \theta .
$$

In what follows we estimate $\xi^{\varepsilon}(t)$. Since

$$
z^{\varepsilon}(t)=e^{-A_{\varepsilon}^{-}\left(t-t_{0}\right)} z^{\varepsilon}\left(t_{0}\right)+\int_{t_{0}}^{t} e^{-A_{\varepsilon}^{-}(t-r)} G_{\varepsilon}\left(v^{\varepsilon}(r), z^{\varepsilon}(r)\right) d r
$$

we have

$$
\begin{aligned}
\xi^{\varepsilon}(t)- & e^{-A_{\varepsilon}^{-}\left(t-t_{0}\right)} \xi^{\varepsilon}\left(t_{0}\right)=z^{\varepsilon}(t)-s_{*}^{\varepsilon}\left(v^{\varepsilon}(t)\right)-e^{-A_{\varepsilon}^{-}\left(t-t_{0}\right)}\left[z^{\varepsilon}\left(t_{0}\right)-s_{*}^{\varepsilon}\left(v^{\varepsilon}\left(t_{0}\right)\right)\right] \\
& =\int_{t_{0}}^{t} e^{-A_{\varepsilon}^{-}(t-r)} G_{\varepsilon}\left(v^{\varepsilon}(r), z^{\varepsilon}(r)\right) d r-s_{*}^{\varepsilon}\left(v^{\varepsilon}(t)\right)+e^{-A_{\varepsilon}^{-}\left(t-t_{0}\right)} s_{*}^{\varepsilon}\left(v^{\varepsilon}\left(t_{0}\right)\right) \\
& =\int_{t_{0}}^{t} e^{-A_{\varepsilon}^{-}(t-r)} G_{\mathcal{\varepsilon}}\left(v^{\varepsilon}(r), z^{\varepsilon}(r)\right) d r-\int_{-\infty}^{t} e^{-A_{\varepsilon}^{-}(t-r)} G_{\mathcal{\varepsilon}}\left(y^{\varepsilon}(r, t), s_{*}^{\varepsilon}\left(y^{\varepsilon}(r, t)\right)\right) d r \\
& +e^{-A_{\varepsilon}^{-}\left(t-t_{0}\right)} \int_{-\infty}^{t_{0}} e^{-A_{\varepsilon}^{-}\left(t_{0}-r\right)} G_{\varepsilon}\left(y^{\varepsilon}\left(r, t_{0}\right), s_{*}^{\varepsilon}\left(y^{\varepsilon}\left(r, t_{0}\right)\right)\right) d r \\
& =\int_{t_{0}}^{t} e^{-A_{\varepsilon}^{-}(t-r)}\left[G_{\mathcal{\varepsilon}}\left(v^{\varepsilon}(r), z^{\varepsilon}(r)\right)-G_{\varepsilon}\left(y^{\varepsilon}(r, t), s_{*}^{\varepsilon}\left(y^{\varepsilon}(r, t)\right)\right)\right] d r \\
& -\int_{-\infty}^{t_{0}} e^{-A_{\varepsilon}^{-}(t-r)}\left[G_{\varepsilon}\left(y^{\varepsilon}(r, t), s_{*}^{\varepsilon}\left(y^{\varepsilon}(r, t)\right)\right)-G_{\varepsilon}\left(y^{\varepsilon}\left(r, t_{0}\right), s_{*}^{\varepsilon}\left(y^{\varepsilon}\left(r, t_{0}\right)\right)\right)\right] d r .
\end{aligned}
$$


Thus,

$$
\begin{aligned}
& \left\|\xi^{\varepsilon}(t)-e^{-A_{\varepsilon}^{-}\left(t-t_{0}\right)} \xi^{\varepsilon}\left(t_{0}\right)\right\|_{X_{\varepsilon}^{\frac{1}{2}}} \\
& \leq \rho M \int_{t_{0}}^{t} e^{-\gamma(t-r)}\left[\left\|v^{\varepsilon}(r)-y^{\varepsilon}(r, t)\right\|_{X_{\varepsilon}^{\frac{1}{2}}}+\left\|z^{\varepsilon}(r)-s_{*}^{\varepsilon}\left(y^{\varepsilon}(r, t)\right)\right\|_{X_{\varepsilon}^{\frac{1}{2}}}\right] d r \\
& +\rho M(1+\Delta) \int_{-\infty}^{t_{0}} e^{-\gamma(t-r)}\left\|y^{\varepsilon}(r, t)-y^{\varepsilon}\left(r, t_{0}\right)\right\|_{X_{\varepsilon}^{\frac{1}{2}}} d r \\
& \leq \rho M \int_{t_{0}}^{t} e^{-\gamma(t-r)}\left\|\xi^{\varepsilon}(r)\right\|_{X_{\varepsilon}^{\frac{1}{2}}} d r \\
& +\rho^{2} M^{2}(1+\Delta) e^{-\gamma t} \int_{t_{0}}^{t} e^{-(-\beta-\rho M(1+\Delta) \theta}\left\|\xi^{\varepsilon}(\theta)\right\|_{X_{\varepsilon}^{\frac{1}{2}}} \int_{-\infty}^{\theta} e^{(\gamma-\beta-\rho M(1+\Delta)) r} d r d \theta \\
& +\rho^{2} M^{3}(1+\Delta) e^{-\gamma t} \int_{t_{0}}^{t} e^{-(-\beta-\rho M(1+\Delta)) \theta}\left\|\xi^{\varepsilon}(\theta)\right\|_{X_{\varepsilon}^{\frac{1}{2}}} \int_{-\infty}^{t_{0}} e^{(\beta-\beta-\rho M(1+\Delta)) r} d r d \theta
\end{aligned}
$$

and then

$$
\begin{aligned}
& \left\|\xi^{\varepsilon}(t)-e^{-A_{\varepsilon}^{-}\left(t-t_{0}\right)} \xi^{\varepsilon}\left(t_{0}\right)\right\|_{X_{\varepsilon}^{\frac{1}{2}}} \leq\left[\rho M-\frac{\rho^{2} M^{2}(1+\Delta)}{\gamma-\beta-\rho M(1+\Delta)}\right] \int_{t_{0}}^{t} e^{-\gamma(t-\theta)}\left\|\xi^{\varepsilon}(\theta)\right\|_{X_{\varepsilon}^{\frac{1}{2}}} d \theta \\
& +\frac{\rho^{2} M^{3}(1+\Delta) e^{-\gamma\left(t-t_{0}\right)}}{\gamma-\beta-\rho M(1+\Delta)} \int_{t_{0}}^{t} e^{-\left(-\beta-\rho M(1+\Delta)\left(\theta-t_{0}\right)\right.}\left\|\xi^{\varepsilon}(\theta)\right\|_{X_{\varepsilon}^{\frac{1}{2}}} d \theta
\end{aligned}
$$

Hence

$$
\begin{aligned}
e^{\gamma\left(t-t_{0}\right)}\left\|\xi^{\varepsilon}(t)\right\|_{X_{\varepsilon}^{\frac{1}{2}}} \leq M\left\|\xi^{\varepsilon}\left(t_{0}\right)\right\|_{X_{\varepsilon}^{\frac{1}{2}}}+\left[\rho M+\frac{\rho^{2} M^{2}(1+\Delta)}{\gamma-\beta-\rho M(1+\Delta)}\right] \int_{t_{0}}^{t} e^{\gamma\left(r-t_{0}\right)}\left\|\xi^{\varepsilon}(r)\right\|_{X_{\varepsilon}^{\frac{1}{2}}} d r \\
+\frac{\rho^{2} M^{3}(1+\Delta)}{\gamma-\beta-\rho M(1+\Delta)} \int_{t_{0}}^{t} e^{-\left(\gamma-\beta-\rho M(1+\Delta)\left(\theta-t_{0}\right)\right.} e^{\beta\left(\theta-t_{0}\right)}\left\|\xi^{\varepsilon}(\theta)\right\|_{X_{\varepsilon}^{\frac{1}{2}}} d \theta \\
\leq M\left\|\xi^{\varepsilon}\left(t_{0}\right)\right\|_{X_{\varepsilon}^{\frac{1}{2}}}+\left[\rho M+\frac{\rho^{2} M^{2}(1+\Delta)(1+M)}{\gamma-\beta-\rho M(1+\Delta)}\right] \int_{t_{0}}^{t} e^{\gamma\left(r-t_{0}\right)}\left\|\xi^{\varepsilon}(r)\right\|_{X_{\varepsilon}^{\frac{1}{2}}} d r .
\end{aligned}
$$

By Gronwall inequality

$$
\left\|\xi^{\varepsilon}(t)\right\|_{X_{\varepsilon}^{\frac{1}{2}}} \leq M\left\|\xi^{\varepsilon}\left(t_{0}\right)\right\|_{X_{\varepsilon}^{\frac{1}{2}}} e^{-(\gamma-L)\left(t-t_{0}\right)}
$$

and then

$$
\left\|z^{\varepsilon}(t)-s_{*}^{\varepsilon}\left(v^{\varepsilon}(t)\right)\right\|_{X_{\varepsilon}^{\frac{1}{2}}}=\left\|\xi^{\varepsilon}(t)\right\|_{X_{\varepsilon}^{\frac{1}{2}}} \leq M\left\|\xi^{\varepsilon}\left(t_{0}\right)\right\|_{X_{\varepsilon}^{\frac{1}{2}}} e^{-(\gamma-L)\left(t-t_{0}\right)} .
$$

Now if $u^{\varepsilon}:=T_{\varepsilon}(t) u_{0}^{\varepsilon}=v^{\varepsilon}(t)+z^{\varepsilon}(t), t \in \mathbb{R}$, denotes the solution through at $u_{0}^{\varepsilon}=v_{0}^{\varepsilon}+z_{0}^{\varepsilon} \in$ $\mathscr{A}_{\mathcal{E}}$, then

$$
\left\|z^{\varepsilon}(t)-s_{*}^{\varepsilon}\left(v^{\varepsilon}(t)\right)\right\|_{X_{\varepsilon}^{\frac{1}{2}}} \leq M\left\|z_{0}^{\varepsilon}-s_{*}^{\varepsilon}\left(v_{0}^{\varepsilon}\right)\right\|_{X_{\varepsilon}^{\frac{1}{2}}} e^{-(\gamma-L)\left(t-t_{0}\right)} .
$$

Since $\left\{T_{\varepsilon}(t) u_{0}^{\varepsilon} ; t \in \mathbb{R}\right\} \subset \mathscr{A}_{\varepsilon}$ is bounded, letting $t_{0} \rightarrow-\infty$ we obtain $T_{\varepsilon}(t) u_{0}^{\varepsilon}=v^{\varepsilon}(t)+s_{*}^{\varepsilon}\left(v^{\varepsilon}(t)\right) \in$ $\mathscr{M}_{\varepsilon}$. That is $\mathscr{A}_{\varepsilon} \subset \mathscr{M}_{\varepsilon}$. Moreover, if $B_{\varepsilon} \subset X_{\varepsilon}^{\frac{1}{2}}$ is a bounded set and $u_{0}^{\varepsilon}=v_{0}^{\varepsilon}+z_{0}^{\varepsilon} \in B_{\varepsilon}$, we conclude that $T_{\mathcal{E}}(t) u_{0}^{\varepsilon}=v^{\varepsilon}(t)+z^{\varepsilon}(t)$ satisfies

$$
\begin{aligned}
\sup _{u_{0}^{\varepsilon} \in B_{\varepsilon}} \inf _{w \in \mathscr{M}_{\varepsilon}}\left\|T_{\mathcal{\varepsilon}}(t) u_{0}^{\varepsilon}-w\right\|_{X_{\varepsilon}^{\frac{1}{2}}} & \leq \sup _{u_{0}^{\varepsilon} \in B_{\varepsilon}}\left\|z^{\varepsilon}(t)-s_{*}^{\varepsilon}\left(v^{\varepsilon}(t)\right)\right\|_{X_{\varepsilon}^{\frac{1}{2}}} \\
& \leq M e^{-(\gamma-L)\left(t-t_{0}\right)} \sup _{u_{0}^{\varepsilon} \in B_{\varepsilon}}\left\|z_{0}^{\varepsilon}-s_{*}^{\varepsilon}\left(v_{0}^{\varepsilon}\right)\right\|_{X_{\varepsilon}^{\frac{1}{2}}}
\end{aligned}
$$


which implies

$$
\operatorname{dist}_{H}\left(T_{\varepsilon}(t) B_{\varepsilon}, \mathscr{M}_{\varepsilon}\right) \leq C\left(B_{\varepsilon}\right) e^{-(\gamma-L)\left(t-t_{0}\right)},
$$

and thus the proof is complete.

Remark 6.4.2. It is well known the $C^{0}, C^{1}$ and $C^{1, \theta}$ convergences of invariant manifolds (see (CARBONE; RUAS-FILHO, 2006) and (SANTAMARIA; ARRIETA, 2014)). That is $\left\|s_{*}^{\varepsilon}-s_{*}^{0}\right\|_{C^{0}\left(Y_{0}\right)},\left\|s_{*}^{\varepsilon}-s_{*}^{0}\right\|_{C^{1}\left(Y_{0}\right)},\left\|s_{*}^{\varepsilon}-s_{*}^{0}\right\|_{C^{1, \theta}\left(Y_{0}\right)} \stackrel{\varepsilon \rightarrow 0}{\longrightarrow} 0$.

\subsection{Rate of Convergence of Attractors}

In this section we will estimate the continuity of attractors of (6.4) in the Hausdorff metric by the rate of convergence of resolvent operators obtained in the Section 6.3.

The operator $A_{\varepsilon}, \varepsilon \in\left[0, \varepsilon_{0}\right]$, has compact resolvent and according to (CARBONE; RUASFILHO, 2006), $A_{0}$ is Sturm-Lioville type, which implies transversality of stable and unstable manifolds of the equilibrium points. Since we assume hyperbolicity, the limiting problem (6.3) generates a Morse-Smale semigroup in $X_{0}^{\frac{1}{2}}$ and hence the perturbed problem (6.1) generates a Morse-Smale semigroup in $X_{\mathcal{\varepsilon}}^{\frac{1}{2}}$.

We saw in the last section how the gap condition implies the existence of the finite dimensional invariant manifold $\mathscr{M}_{\varepsilon}$ for (6.4). The invariant manifold contains the attractor $\mathscr{A}_{\varepsilon}$ and the flow is given by an ordinary differential equation. That is,

$$
u^{\varepsilon}(t)=v^{\varepsilon}(t)+s_{*}^{\varepsilon}\left(v^{\varepsilon}(t)\right), \quad t \in \mathbb{R},
$$

where $v^{\varepsilon}(t)$ satisfies

$$
\dot{v}^{\varepsilon}+A_{\varepsilon}^{+} v^{\varepsilon}=Q_{\varepsilon} f\left(v^{\varepsilon}+s_{*}^{\varepsilon}\left(v^{\varepsilon}(t)\right)\right),
$$

and we can consider $v^{\varepsilon} \in \mathbb{R}^{m}$ and $H_{\varepsilon}\left(v^{\varepsilon}\right)=Q_{\varepsilon} f\left(v^{\varepsilon}+s_{*}^{\varepsilon}\left(v^{\varepsilon}(t)\right)\right)$ a continuously differentiable map in $\mathbb{R}^{m}$. For each $\varepsilon \in\left[0, \varepsilon_{0}\right]$, we denote $\tilde{T}_{\varepsilon}=\tilde{T}_{\mathcal{\varepsilon}}(1)$, where $\tilde{T}_{\varepsilon}(\cdot)$ is the semigroup generated by solution $v^{\varepsilon}(\cdot)$ of (6.11) in $\mathbb{R}^{m}$. We have the following convergences

$$
\left\|\tilde{T}_{\varepsilon}-\tilde{T}_{0}\right\|_{C^{1}\left(\mathbb{R}^{m}, \mathbb{R}^{m}\right)} \stackrel{\varepsilon \rightarrow 0}{\longrightarrow} 0 \quad \text { and } \quad\left\|\tilde{T}_{\varepsilon}-\tilde{T}_{0}\right\|_{L^{\infty}\left(\mathbb{R}^{m}, \mathbb{R}^{m}\right)} \leq C \tau(\varepsilon)|\log (\tau(\varepsilon))|
$$

where $\tau(\varepsilon)=\left(\left\|p_{\varepsilon}-p_{0}\right\|_{L^{\infty}\left(\Omega_{1}\right)}+\varepsilon\right)^{\frac{1}{2}}$ and the last estimate is proved as Theorem 6.2.3.

Now we are ready to prove the main result of this chapter.

Theorem 6.5.1. Let $\mathscr{A}_{\mathcal{E}}, \varepsilon \in\left[0, \varepsilon_{0}\right]$, be the attractor for (6.4). Then there is a positive constant $C$ independent of $\varepsilon$ such that

$$
\mathrm{d}_{H}\left(\mathscr{A}_{\varepsilon}, \mathscr{A}_{0}\right) \leq C\left(\left\|p_{\varepsilon}-p_{0}\right\|_{L^{\infty}\left(\Omega_{1}\right)}+\varepsilon\right)^{\frac{1}{2}}\left|\log \left(\left\|p_{\varepsilon}-p_{0}\right\|_{L^{\infty}\left(\Omega_{1}\right)}+\varepsilon\right)\right| .
$$


Proof. We will follow (SANTAMARIA; ARRIETA, 2014). For each $\varepsilon \in\left[0, \varepsilon_{0}\right]$ we denote $T_{\varepsilon}=T_{\varepsilon}(1)$. Given $u^{\varepsilon} \in \mathscr{A}_{\varepsilon}$, by invariance there is $w^{\varepsilon} \in \mathscr{A}_{\varepsilon}$ such that $u^{\varepsilon}=T_{\varepsilon} w^{\varepsilon}$ so we can write $w^{\varepsilon}=Q_{\varepsilon} w^{\varepsilon}+s_{*}^{\varepsilon}\left(Q_{\varepsilon} w^{\varepsilon}\right)$, where $Q_{\varepsilon} w^{\varepsilon} \in \overline{\mathscr{A}}_{\varepsilon}$ with $\overline{\mathscr{A}}_{\varepsilon}=Q_{\varepsilon} \mathscr{A}_{\varepsilon}$ the projected attractor in $\mathbb{R}^{m}$. Thus

$$
\begin{aligned}
\left\|u^{\varepsilon}-u^{0}\right\|_{X_{\varepsilon}^{\frac{1}{2}}} & =\left\|T_{\varepsilon} w^{\varepsilon}-T_{0} w^{0}\right\|_{X_{\varepsilon}^{\frac{1}{2}}} \leq\left\|T_{\varepsilon} w^{\varepsilon}-T_{\varepsilon} w^{0}\right\|_{X_{\varepsilon}^{\frac{1}{2}}}+\left\|T_{\varepsilon} w^{0}-T_{0} w^{0}\right\|_{X_{\varepsilon}^{\frac{1}{2}}} \\
& \leq C\left(\left\|p_{\varepsilon}-p_{0}\right\|_{L^{\infty}\left(\Omega_{1}\right)}+\varepsilon\right)^{\frac{1}{2}}\left|\log \left(\left\|p_{\varepsilon}-p_{0}\right\|_{L^{\infty}\left(\Omega_{1}\right)}+\varepsilon\right)\right|+C\left\|w^{\varepsilon}-w^{0}\right\| .
\end{aligned}
$$

But

$$
\begin{aligned}
& \left\|w^{\varepsilon}-w^{0}\right\|=\left\|Q_{\varepsilon} w^{\varepsilon}-Q_{0} w^{0}\right\|_{\mathbb{R}^{m}}+\left\|s_{*}^{\varepsilon}\left(Q_{\varepsilon} w^{\varepsilon}\right)-s_{*}^{0}\left(Q_{0} w^{0}\right)\right\|_{X_{\varepsilon}^{\frac{1}{2}}} \\
& \quad \leq\left\|Q_{\varepsilon} w^{\varepsilon}-Q_{0} w^{0}\right\|_{\mathbb{R}^{m}}+\left\|s_{*}^{\varepsilon}\left(Q_{\varepsilon} w^{\varepsilon}\right)-s_{*}^{\varepsilon}\left(Q_{0} w^{0}\right)\right\|_{X_{\varepsilon}^{\frac{1}{2}}}+\left\|s_{*}^{\varepsilon}\left(Q_{0} w^{0}\right)-s_{*}^{0}\left(Q_{0} w^{0}\right)\right\|_{X_{\varepsilon}^{\frac{1}{2}}} \\
& \quad \leq C\left\|Q_{\varepsilon} w^{\varepsilon}-Q_{0} w^{0}\right\|_{\mathbb{R}^{m}}+C\left(\left\|p_{\varepsilon}-p_{0}\right\|_{L^{\infty}\left(\Omega_{1}\right)}+\varepsilon\right)^{\frac{1}{2}}\left|\log \left(\left\|p_{\varepsilon}-p_{0}\right\|_{L^{\infty}\left(\Omega_{1}\right)}+\varepsilon\right)\right|,
\end{aligned}
$$

which implies

$$
\mathrm{d}_{H}\left(\mathscr{A}_{\varepsilon}, \mathscr{A}_{0}\right) \leq \mathrm{d}_{H}\left(\overline{\mathscr{A}}_{\varepsilon}, \overline{\mathscr{A}}_{0}\right)+C\left(\left\|p_{\varepsilon}-p_{0}\right\|_{L^{\infty}\left(\Omega_{1}\right)}+\varepsilon\right)^{\frac{1}{2}}\left|\log \left(\left\|p_{\varepsilon}-p_{0}\right\|_{L^{\infty}\left(\Omega_{1}\right)}+\varepsilon\right)\right| .
$$

The result follows by (6.12) and Theorem 2.5.10. 
ARAGAO-COSTA, E.; CARABALlO, T.; CARVALHO, J. A. N. Stability of gradient semigroups under perturbations. Nonlinearity, v. 24, 2011. Cited on page 45.

ARRIETA, J. M.; BEZERRA, F. D.; CARVALHO, A. N. Rate of convergence of attractors for some singular perturbed parabolic problems. Top. Methdos Nonlinear Anal, 2013. Cited 7 times on pages $19,43,46,52,79,120$, and 122 .

ARRIETA, J. M.; CARVALHO, A. N.; RODRíGUEZ-BERNAL, A. Parabolic problems with nonlinear boundary conditions and critical nonlinearities. Journal of Differential Equations, v. 156, p. 376-406, 1999. Cited 4 times on pages 76, 82, 101, and 116.

Attractors for parabolic problems with nonlinear boundary bondition. uniform bounds. Commun. in partial differential equations, v. 25, p. 1-37, 2000. Cited 5 times on pages 76, 82, 83, 101, and 116 .

Upper semicontinuity for attractors of parabolic problems with localized large diffusion and nonlinear boundary conditions. Journal of Differential Equations, v. 168, p. 33-59, 2000. Cited 5 times on pages 19, 101, 113, 116, and 117.

BABIN, A.; VISHIK, M. Attractors of Evolution Equations. [S.1.]: Elsevier, 1992. Cited 4 times on pages $19,20,27$, and 52 .

BERNAL, A. R. On the construction of initial manifold under simmetry constraints ii: $\mathrm{O}$ constraint and inertial manifolds on thin domains. J. Math. Pures App., v. 79, p. 57-79, 1993. Cited on page 51.

BORTOLAN, M. C.; CARVALHO, A. N.; LANGA, J. A.; RAUGEL, G. Non-autonomous perturbations of morse-smale semigroups: stability of the phase diagram. Preprint, 2015. Cited 6 times on pages $43,45,52,80,87$, and 117 .

BREZIS, H. Functional Analysis, Sobolev Spaces and Partial Differential Equations. [S.1.]: Springer, 2010. Cited on page 81.

BRUNOVSKÝ, P.; POLÁCIK, P. The morse-smale sctruture of a generic reaction-diffusion equation in higher space dimension. J. Differential Equations, v. 135, p. 129-181, 1997. Cited on page 87.

CARBONE, V. L.; CARVALHO, A. N.; SCHIABEL-SILVA, K. Continuity of attractors for parabolic problems with localized large diffusion. Nonlinear Analysis: Theory, Methods and Applications, v. 68, n. 3, p. 515-535, 2008. Cited 6 times on pages 19, 79, 113, 117, 122, and 123.

CARBONE, V. L.; RUAS-FILHO, J. G. Continuity of the attractors in a singular problem arising in composite materials. Nonlinear Anal, v. 65, p. 1285-1305, 2006. Cited 6 times on pages 46, $117,122,123,124$, and 131. 
CARVALHO, A.; CHOLEWA, J.; T.DLOTKO. Equi-exponential attraction and rate of convergence of attractors for singularly pertubed evolution equations. Cadernos de matemática, v. 11, p. 111-151, 2010. Cited on page 42 .

CARVALHO, A.; PISKAREV, S. A general approximation scheme for attractors of abstract parabolic problems. Numer Funct Anal Optim, v. 27, p. 785-829, 2006. Cited 3 times on pages 87,89 , and 122 .

CARVALHO, A. N.; CHOLEWA, J. W.; LOZADA-CRUZ, G.; PRIMO, M. R. Reduction of infinite dimensional systems to finite dimensions: Compact convergence approach. SIAM J. Math. Anal., v. 45, n. 2, p. 600-638, 2010. Cited 6 times on pages 51, 72, 81, 89, 102, and 124.

CARVALHO, A. N.; LANGA, J.; ROBINSON, J. Attractors for infinite-dimensional nonautonomous dynamical systems. [S.1.]: Springer, 2010. Cited 6 times on pages 27, 31, 53, 82, 86, and 90.

CARVALHO, A. N.; PEREIRA, A. L. A sacalar parabolic equation whose asymptotic behavior is dictated by a system of ordinary differential equations. J Differential Equations, v. 112, n. 81-130, 1994. Cited 5 times on pages 51, 91, 92, 93, and 113.

CARVALHO, A. N.; PIRES, L. Rate of convergence of attractors for semilinear singularly perturbed problems: parabolic equations with large diffusion. Preprint, 2016. Cited on page 116.

CARVALHO, A. N.; PRIMO, M. R. Spatial homogeneity in parabolic problems with nonlinear boundary conditions. Commun. Pure Appl. Anal., v. 3, p. 637-651, 2004. Cited 2 times on pages 51 and 82 .

CONLEY, E.; HOFF, D.; SMOLLER, J. Time behaviour of solutions of systems of nonlinear reaction-diffusion equations. SIAM J. Appl. Math., v. 35, p. 1-16, 1978. Cited 2 times on pages 51 and 73 .

FUSCO, G. On the explicit construction of an ode which has the same dynamics as a scalar parabolic pde. J. Differential Equations, v. 69, p. 85-110, 1987. Cited 4 times on pages 51, 91, 92, and 113.

HALE, J. Large diffusivity and asymptotic behavior in parabolic systems. J. Math. Analysis Applicable, v. 118, p. 455-466, 1986. Cited 3 times on pages 51, 73, and 85.

Asymptotic behavior of dissipative systems. [S.1.]: Mathematical surveys and monographs, 1988. Cited 3 times on pages 27, 33, and 76.

Ordinary Differential Equations. [S.1.]: Dover Publications, 2009. Cited 2 times on pages 28 and 53.

HALE, J.; RAUGEL, G. Upper-semicontinuous of the attractor for a singularity perturbed hyperbolic equation. J. Diff. Equat., v. 73, p. 197-214, 1988. Cited on page 19.

. Lower semicontinuity of attractors of gradient systems and applications. Annali di Matematica Pura ed Applicata, v. 154, p. 281-326, 1989. Cited on page 19.

Lower semicontinuity of the attractor for a singularity perturbed hyperbolic equation. Journal of Dynamics and Differential Equations, v. 2, p. 19-67, 1990. Cited on page 19. 
HENRY, D. Geometric theory of semilinear parabolic equations. [S.1.]: Lecture Notes in Mathematics, 1980. (Springer-Velag, 840). Cited 10 times on pages 22, 27, 36, 51, 75, 81, 82, 92, 116, and 121.

Some infinite-dimensional morse-smale systems defined by parabolic partial differential equations. Journal of differential equations, v. 59, p. 165-205, 1985. Cited 3 times on pages 37,44 , and 46.

Exponential dichotomies, the shadowing lemma and homoclinic orbits in banach spaces. Resenhas IME-USP, v. 1, n. 4, p. 381-401, 1994. Cited on page 48.

Semigroups. IME-USP-São Paulo- Brasil disponível em http://www.ime.usp.br/map/dhenry/danhenry/main.htm, 1994. Cited on page 28.

KATO, T. Perturbation Theory for Linear Operators. [S.1.]: Springer,Berlin, 1980. Cited 3 times on pages 58,83 , and 89 .

PILYUGUN, S. Y. Shadowing in Dynamical Systems. [S.1.]: Springer, 1999. (Lectures Notes in Mathematics, v. 1706). Cited on page 48.

ROBINSON, J. Infinite-dimensional dynamical systems. [S.1.]: Cambridge University Press, 2001. Cited 2 times on pages 27 and 32.

RODRíGUEZ-BERNAL, A. Localized spatial homogenizations and large diffusion. SIAM Journal of Mathematical Analysis, v. 29, n. 6, p. 1361-1380, 1998. Cited 4 times on pages $113,115,121$, and 122 .

RODRíGUEZ-BERNAL, A.; WILLIE, R. Singular large diffusivity and spatial homogenization in a non homogeneous linear parabolic problem. Discrete and Continuous Dynamical Systems. Series B. A Journal Bridging Mathematics and Sciences, v. 5, n. 2, p. 385-410, 2005. Cited 2 times on pages 74 and 75 .

SANTAMARIA, E.; ARRIETA, J. M. Estimates on the distance of inertial manifolds. Discrete and continuous dynamical systems, v. 34, n. 10, p. 3921-3944, 2014. Cited 16 times on pages 20, 21, 24, 46, 48, 49, 52, 66, 67, 86, 117, 120, 121, 128, 131, and 132.

SELL, G.; YOU, Y. Dynamics of Evolution Equations. [S.1.]: Applied Mathematical Sciences, Springer, 2002. Cited 2 times on pages 51 and 66. 